\title{
a-Functionalization of Cyclic Secondary Amines: Lewis Acid Promoted Addition of Organometallics to Transient Imines
}

\author{
Anirudra Paul and Daniel Seidel* \\ Center for Heterocyclic Compounds, Department of Chemistry, University of Florida, \\ Gainesville, Florida 32611, United States
}

\section{Supporting Information}

\section{Table of Contents}

General Information

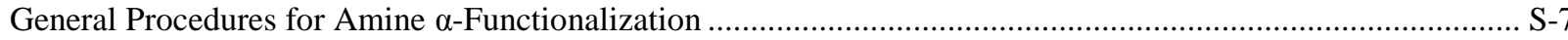

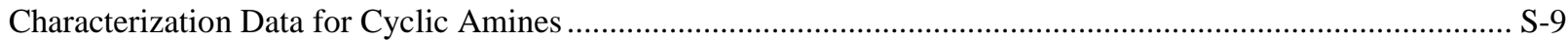

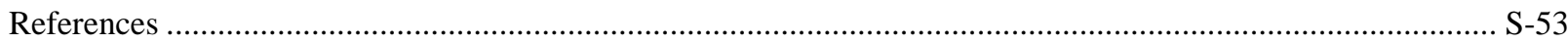

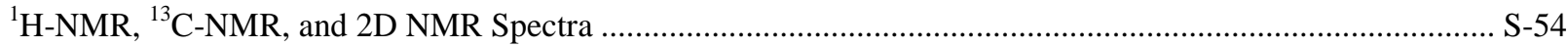


General Information: Starting materials and reagents were purchased from commercial sources and used as received unless stated otherwise. Anhydrous THF, diethyl ether and dichloromethane were dried using a JC Meyer solvent system. All liquid amines and trifluoroacetophenone were distilled prior to use. Benzophenone was recrystallized from hexanes/EtOAc. $n$-BuLi solution in hexanes was purchased from commercial sources and freshly titrated using $N$-pivaloyl-o-toluidine prior to use. ${ }^{1}$ All commercial Grignard reagents were freshly titrated prior to use. Purification of reaction products was carried out by flash column chromatography using Sorbent Technologies Standard Grade silica gel (60 ̊, 230-400 mesh). Analytical thin layer chromatography was performed on EM Reagent $0.25 \mathrm{~mm}$ silica gel 60 F254 plates. Visualization was accomplished with UV light or Dragendorff-Munier stains, followed by heating. Proton nuclear magnetic resonance spectra $\left({ }^{1} \mathrm{H}\right.$ NMR $)$ were recorded on a Varian Unity Inova $500 \mathrm{MHz}$ or Varian VNMRS-400 MHz instrument and chemical shifts are reported in ppm using the solvent as an internal standard $\left(\mathrm{CDCl}_{3}\right.$ at $\left.7.26 \mathrm{ppm}\right)$. Data are reported as app $=$ apparent, $\mathrm{s}=$ singlet, $\mathrm{d}=$ doublet, $\mathrm{t}=$ triplet, $\mathrm{q}=$ quartet, $\mathrm{m}=$ multiplet, comp = complex, $\mathrm{br}=$ broad; coupling constant(s) in Hz. Proton-decoupled carbon nuclear magnetic resonance spectra $\left({ }^{13} \mathrm{C} N M R\right)$ spectra were recorded on a Varian Unity Inova $500 \mathrm{MHz}$ or Varian VNMRS-400 MHz instrument and chemical shifts are reported in ppm using the solvent as an internal standard $\left(\mathrm{CDCl}_{3}\right.$ at $\left.77.0 \mathrm{ppm}\right)$. High resolution mass spectra (HRMS) were obtained from the Mass Spectrometry Core Laboratory of the University of Florida (Agilent 6200 ESI-TOF coupled to an IonSense DART ET-100 ionization source for DART-TOF analysis) and are reported as $\mathrm{m} / \mathrm{z}$ (relative ratio). Accurate mass data (ESI) were obtained from an Agilent 1260 Infinity II LC/MSD instrument using MassWorks 5.0 from CERNO bioscience. $^{2}$ Amines $\mathbf{7 a}, \mathbf{7 c}$, and 7o were previously reported and their published characterization data matched our own in all respects. ${ }^{3}$ But-3-en-1-ylmagnesium bromide was prepared according to a literature procedure. ${ }^{4}$ 


\section{Evaluation of Reaction Conditions for the $\alpha-\mathrm{C}-\mathrm{H}$ Bond Functionalization of Pyrrolidine with Lithium Phenylacetylide}

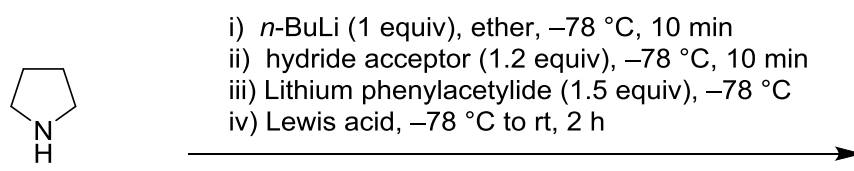

i) $n$-BuLi ( 1 equiv), ether, $-78^{\circ} \mathrm{C}, 10 \mathrm{~min}$

iii) Lithium phenylacetylide ( 1.5 equiv) $-78^{\circ} \mathrm{C}$

iv) Lewis acid, $-78^{\circ} \mathrm{C}$ to $\mathrm{rt}, 2 \mathrm{~h}$

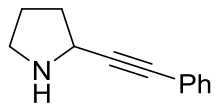

$( \pm)-5 a$

\begin{tabular}{cccc}
\hline Entry & Hydride Acceptor & $\begin{array}{c}\text { Lewis Acid } \\
\text { (equiv) }\end{array}$ & Yield (\%) \\
\hline 1 & Benzophenone & - & $\mathrm{NR}$ \\
2 & Benzophenone & $\mathrm{BF}_{3} \cdot \mathrm{OEt}_{2}(0.6)$ & 22 \\
3 & Benzophenone & $\mathrm{BF}_{3} \cdot \mathrm{OEt}_{2}(1)$ & 48 \\
4 & Benzophenone & $\mathrm{BF}_{3} \cdot \mathrm{OEt}_{2}(1.2)$ & 54 \\
$5^{\mathrm{a}}$ & Benzophenone & $\mathrm{BF}_{3} \cdot \mathrm{OEt}_{2}(1.2)$ & 56 \\
6 & Benzophenone & $\mathrm{BF}_{3} \cdot \mathrm{OEt}_{2}(2.0)$ & 30 \\
$7^{\mathrm{b}}$ & Benzophenone & $\mathrm{BF}_{3} \cdot \mathrm{OEt}_{2}(1.2)$ & 36 \\
8 & Benzophenone & $\mathrm{TMSOTf}^{\mathrm{b}}(1.2)$ & 34 \\
9 & Trifluoroacetophenone & $\mathrm{BF}_{3} \cdot \mathrm{OEt}_{2}(1.2)$ & 49 \\
$10^{\mathrm{c}}$ & Trifluoroacetophenone & $\mathrm{BF}_{3} \cdot \mathrm{OEt}_{2}(1.2)$ & 14 \\
11 & Trifluoroacetophenone & $\mathrm{BF}_{3} \cdot \mathrm{OEt}_{2}(2.2)$ & 48 \\
$12^{\mathrm{d}}$ & Trifluoroacetophenone & $\mathrm{BF}_{3} \cdot \mathrm{OEt}_{2}(2.2)$ & $\mathrm{NR}$ \\
$13^{\mathrm{e}}$ & Trifluoroacetophenone & $\mathrm{BF}_{3} \cdot \mathrm{OEt}_{2}(1.2)$ & $\mathrm{NR}$ \\
$14^{\mathrm{f}}$ & Trifluoroacetophenone & $\mathrm{BF}_{3} \cdot \mathrm{OEt}_{2}(1.2)$ & 40 \\
\hline
\end{tabular}

Reactions were performed on a $1 \mathrm{mmol}$ scale. Pyrrolidine was dissolved in $2 \mathrm{~mL}$ of ether and the hydride acceptor was dissolved in $1 \mathrm{~mL}$ of ether. Lithium phenylacetylide was prepared in THF. All reactions were performed at a $0.2 \mathrm{M}$ final concentration of the limiting reagent (pyrrolidine). Unless otherwise specified, the Lewis acid was added last to a solution of imine immediately after the addition of the lithiate. " $-78^{\circ} \mathrm{C}$ to $\mathrm{rt}$ " indicates that the reaction vessel was taken out of the low temperature bath following the addition of the lithiate. The reaction mixture was then stirred for the time indicated. All yields correspond to isolated yields of purified products. NR: no reaction

${ }^{\text {a }}$ Lithium phenylacetylide was prepared in a 4:1 mixture of PhMe and THF for $30 \mathrm{~min}$ at $-78{ }^{\circ} \mathrm{C}$.

b The reaction was conducted at $-78^{\circ} \mathrm{C}$ for $6 \mathrm{~h}$.

${ }^{\mathrm{c}}$ Lewis acid and lithium phenylacetylide were premixed for $15 \mathrm{~min}$ at $-78^{\circ} \mathrm{C}$.

d The Lewis acid was added to the imine solution at $-78{ }^{\circ} \mathrm{C}$ and stirred for $15 \mathrm{~min}$, followed by the addition of the lithiate.

e The imine was prepared in THF.

${ }^{\mathrm{f}}$ The lithiate was prepared in ether. 
Evaluation of Reaction Conditions for the $\alpha-\mathrm{C}-\mathrm{H}$ Bond Functionalization of Azacyclotridecane with Butylmagnesium Chloride
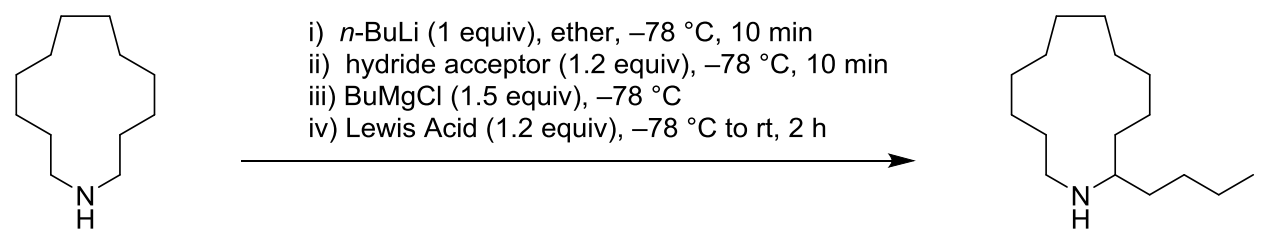

$( \pm)-7 h$

\begin{tabular}{cccc}
\hline Entry & Lewis Acid & Hydride Acceptor & Yield (\%) \\
\hline 1 & - & Trifluoroacetophenone & NR \\
2 & $\mathrm{TMSOTf}$ & Trifluoroacetophenone & 87 \\
3 & $\mathrm{BF}_{3} \cdot \mathrm{OEt}_{2}$ & Trifluoroacetophenone & 73 \\
4 & $\mathrm{BF}_{3} \cdot \mathrm{OEt}_{2}$ & Benzophenone & 40 \\
5 & $\mathrm{TMSOTf}$ & Benzophenone & 46 \\
\hline
\end{tabular}

Reactions were performed on a $0.5 \mathrm{mmol}$ scale. Azacyclotridecane was dissolved in $1 \mathrm{~mL}$ of ether and the hydride acceptor was dissolved in $0.5 \mathrm{~mL}$ of ether. All reactions were performed at a $0.25 \mathrm{M}$ final concentration of the limiting reagent (azacyclotridecane). The Lewis acid was added last to a solution of imine immediately after the addition of the Grignard reagent. " $-78^{\circ} \mathrm{C}$ to rt" indicates that the reaction vessel was taken out of the low temperature bath following the addition of the lithiate. The reaction mixture was then stirred for $2 \mathrm{~h}$. All yields correspond to isolated yields of purified products. NR: no reaction 


\section{Evaluation of Reaction Conditions for the $\alpha-C-H$ Bond Functionalization of Pyrrolidine with Phenylmagnesium Bromide}

i) $n$-BuLi (1 equiv), ether, $-78^{\circ} \mathrm{C}, 10 \mathrm{~min}$
ii) hydride Acceptor (1.2 equiv), $-78^{\circ} \mathrm{C}, 10 \mathrm{~min}$
iii) $\mathrm{PhMgBr}\left(1.5\right.$ equiv), $-78^{\circ} \mathrm{C} \mathrm{C}$
iv) Lewis Acid (1.2 equiv), $-78^{\circ} \mathrm{C}$ to rt, $2 \mathrm{~h}$

Reactions were performed in $1 \mathrm{mmol}$ scale. Pyrrolidine was dissolved in $2 \mathrm{~mL}$ of ether and the hydride acceptor was dissolved in $1 \mathrm{~mL}$ of ether. All reactions were performed in $0.25 \mathrm{M}$ final concentration of the limiting reagent. The Lewis acid was added last to a solution of imine immediately after the addition of the Grignard reagent. " $-78{ }^{\circ} \mathrm{C}$ to $\mathrm{rt}$ " indicates that the reaction vessel was taken out of the low temperature bath following the addition of the lithiate. The reaction mixture was then stirred for $2 \mathrm{~h}$. All yields correspond to isolated yields of purified products. NR: no reaction 
Evaluation of Reaction Conditions for the $\alpha-\mathrm{C}-\mathrm{H}$ Bond Functionalization of Piperidine via the Turbo Grignard Method

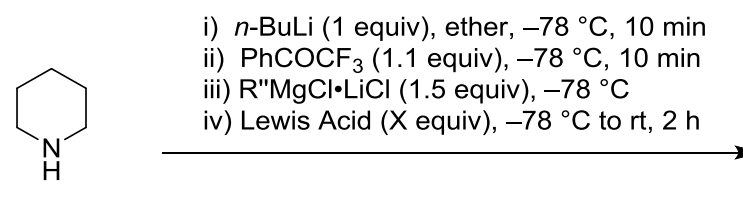

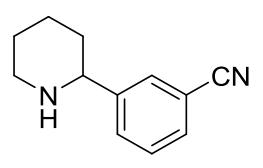

$( \pm)-\mathbf{8 d}$

\begin{tabular}{ccc}
\hline Entry & Lewis Acid & Yield (\%) \\
\hline 1 & - & $\mathrm{NR}$ \\
2 & $\mathrm{TMSOTf}^{(1.2)}$ & 30 \\
3 & $\mathrm{BF}_{3} \cdot \mathrm{OEt}_{2}(1.2)$ & 52 \\
4 & $\mathrm{BF}_{3} \cdot \mathrm{OEt}_{2}(2.2)$ & 40 \\
\hline
\end{tabular}

Reactions were performed in $1 \mathrm{mmol}$ scale. Piperidine was dissolved in $2 \mathrm{~mL}$ of ether and the hydride acceptor was dissolved in $1 \mathrm{~mL}$ of ether. All reactions were performed in $0.2 \mathrm{M}$ final concentration of the limiting reagent. The Lewis acid was added last to a solution of imine immediately after the addition of the Grignard reagent. " $-78^{\circ} \mathrm{C}$ to rt" indicates that the reaction vessel was taken out of the low temperature bath following the addition of the lithiate. The reaction mixture was then stirred for $2 \mathrm{~h}$. All yields correspond to isolated yields of purified products. NR: no reaction 
General Procedure A for the $\alpha-C-H$ Bond Functionalization of Unsubstituted Cyclic Amines with Organometallics:

To a solution of the amine ( $1 \mathrm{mmol}, 1$ equiv) in anhydrous ether $(2 \mathrm{~mL})$ cooled to $-78{ }^{\circ} \mathrm{C}$ was slowly added $n$-BuLi in hexanes ( $1 \mathrm{mmol}, 1$ equiv) under the protection of nitrogen, and the resulting solution was stirred at the same temperature for $10 \mathrm{~min}$. To this was then added via cannula a solution of the corresponding hydride acceptor in anhydrous ether $(1 \mathrm{~mL})$. The resulting mixture was stirred at $-78{ }^{\circ} \mathrm{C}$ for $10 \mathrm{~min}$, followed by the addition of the organometallic nucleophile ( $1.5 \mathrm{mmol}, 1.5$ equiv) in one portion, followed immediately by the addition of the Lewis acid (1.2 mmol, 1.2 equiv). Subsequently, the reaction vessel was taken out of the low temperature bath and stirred at room temperature for $2 \mathrm{~h}$. The reaction mixture was then cooled to $0{ }^{\circ} \mathrm{C}$ and quenched via the addition of methanol $(0.5 \mathrm{~mL})$. The resulting mixture was diluted with ether $(20 \mathrm{~mL})$ and washed with $1 \mathrm{M} \mathrm{NaOH}$ solution $(20$ $\mathrm{mL})$. The aqueous layer was then extracted with ether $(3 \times 20 \mathrm{~mL})$ and the combined organic layers washed with brine $(30 \mathrm{~mL})$ and dried over anhydrous $\mathrm{Na}_{2} \mathrm{SO}_{4}$. Solvent was then removed under reduced pressure and the residue purified by silica gel chromatography.

\section{General Procedure $B$ for the $\alpha-C-H$ Bond Functionalization of Unsubstituted Cyclic Amines with Organometallics:}

To a solution of the amine $\left(0.5 \mathrm{mmol}, 1\right.$ equiv) in anhydrous ether $(1 \mathrm{~mL})$ cooled to $-78{ }^{\circ} \mathrm{C}$ was slowly added $n$ $\mathrm{BuLi}$ in hexanes $(0.5 \mathrm{mmol}, 1$ equiv) under the protection of nitrogen, and the resulting solution was stirred at the same temperature for $10 \mathrm{~min}$. To this was then added via cannula a solution of the corresponding hydride acceptor in anhydrous ether $(0.5 \mathrm{~mL})$. The resulting mixture was stirred at $-78^{\circ} \mathrm{C}$ for $10 \mathrm{~min}$, followed by the addition of the organometallic nucleophile $(0.75 \mathrm{mmol}, 1.5$ equiv) in one portion, followed immediately by the addition of the Lewis acid (0.6 mmol, 1.2 equiv). Subsequently, the reaction vessel was taken out of the low temperature bath and stirred at room temperature for $2 \mathrm{~h}$. The reaction mixture was then cooled to $0{ }^{\circ} \mathrm{C}$ and quenched via the addition of methanol $(0.25 \mathrm{~mL})$. The resulting mixture was diluted with ether $(10 \mathrm{~mL})$ and washed with $1 \mathrm{M} \mathrm{NaOH}$ solution $(10 \mathrm{~mL})$. The aqueous layer was then extracted with ether $(3 \times 10 \mathrm{~mL})$ and the combined organic layers washed with brine $(10 \mathrm{~mL})$ and dried over anhydrous $\mathrm{Na}_{2} \mathrm{SO}_{4}$. Solvent was then removed under reduced pressure and the residue purified by silica gel chromatography.

\section{General Procedure C for the Preparation of Lithium Acetylides:}

To a solution of the alkyne (1.5 mmol, 1.5 equiv to the amine) in anhydrous toluene (1.6 mL) and anhydrous THF $(0.4 \mathrm{~mL})$ cooled to $-78{ }^{\circ} \mathrm{C}$ was slowly added $n$-BuLi in hexanes (1.5 mmol, 1.5 equiv to the amine) under the protection of nitrogen. The resulting mixture was stirred at the same temperature for 30 min to give the corresponding lithium acetylide solution.

\section{General Procedure D for the Preparation of Lithium Acetylides:}

To a solution of the alkyne ( $1.5 \mathrm{mmol}, 1.5$ equiv to the amine) in anhydrous $\mathrm{THF}(2 \mathrm{~mL})$ cooled to $-78{ }^{\circ} \mathrm{C}$ was slowly added $n$-BuLi in hexanes ( $1.5 \mathrm{mmol}, 1.5$ equiv to the amine) under the protection of nitrogen. The resulting mixture was stirred at the same temperature for $30 \mathrm{~min}$ to give the corresponding lithium acetylide solution. 


\section{General Procedure E for the $\alpha-C-H$ Bond Functionalization of Unsubstituted Cyclic Amines with Lithiated N-methyl indole:}

To a solution of 1-methyl-1H-indole ( $196.6 \mathrm{mg}, 1.5 \mathrm{mmol}, 1.5$ equiv to the amine) in anhydrous THF ( $2 \mathrm{~mL})$ cooled to $-78{ }^{\circ} \mathrm{C}$ was added slowly $n$-BuLi in hexanes $(1.5 \mathrm{mmol}, 1.5$ equiv to the amine) under the protection of nitrogen. The resulting mixture was stirred at the same temperature for $5 \mathrm{~min}$ after which it was brought to room temperature over $30 \mathrm{~min}$ to give the corresponding lithiated indole solution which was then cooled back down to $-78^{\circ} \mathrm{C}$. To a separate dry round-bottom flask charged with the corresponding cyclic amine ( $1 \mathrm{mmol}, 1$ equiv) was added anhydrous ether $(2 \mathrm{~mL})$. The solution was cooled to $-78{ }^{\circ} \mathrm{C}$ and $n$-BuLi in hexanes ( $1 \mathrm{mmol}, 1$ equiv) was added dropwise. To this was then added via cannula a solution of the corresponding hydride acceptor in anhydrous ether $(1 \mathrm{~mL})$. The resulting mixture was stirred at $-78{ }^{\circ} \mathrm{C}$ for $10 \mathrm{~min}$. The imine solution was then taken up by syringe and added in one portion to the stirred lithiated indole solution at $-78{ }^{\circ} \mathrm{C}$ followed immediately by the addition of anhydrous $\mathrm{BF}_{3} \cdot \mathrm{OEt}_{2}(148 \mu \mathrm{L}, 1.2 \mathrm{mmol}, 1.2$ equiv). Subsequently, the reaction vessel was taken out of the low temperature bath and stirred at room temperature for $2 \mathrm{~h}$. The reaction mixture was then cooled to $0{ }^{\circ} \mathrm{C}$ and quenched via the addition of methanol $(0.25 \mathrm{~mL})$. The resulting mixture was diluted with ether $(10 \mathrm{~mL})$ and washed with $1 \mathrm{M} \mathrm{NaOH}$ solution $(10 \mathrm{~mL})$. The aqueous layer was then extracted with ether $(3 \times 10 \mathrm{~mL})$ and the combined organic layers washed with brine $(10 \mathrm{~mL})$ and dried over anhydrous $\mathrm{Na}_{2} \mathrm{SO}_{4}$. Solvent was then removed under reduced pressure and the residue purified by silica gel chromatography. 
2-(Phenylethynyl)pyrrolidine

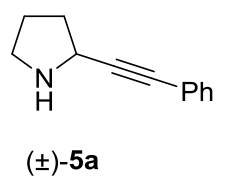

Following general procedures $\mathrm{A}$ and $\mathrm{C}$, compound ( \pm )-5a was obtained from pyrrolidine $(82 \mu \mathrm{L}, 1 \mathrm{mmol})$, benzophenone $(219 \mathrm{mg}, 1.2 \mathrm{mmol})$, ethynylbenzene $(153 \mathrm{mg}, 1.5 \mathrm{mmol})$, and $\mathrm{BF}_{3} \bullet \mathrm{OEt}_{2}(148 \mu \mathrm{L}, 1.2 \mathrm{mmol})$. Compound ( \pm )-5a was obtained as a colorless oil in $56 \%$ yield $(95.8 \mathrm{mg})$.

\section{Characterization data:}

$\mathbf{R}_{\mathbf{f}}=0.22$ in $\left.\mathrm{EtOAc} / \mathrm{MeOH} / i-\mathrm{PrNH}_{2} 90: 9: 1 \mathrm{v} / \mathrm{v} / \mathrm{v}\right)$

${ }^{1}$ H-NMR $\left(500 \mathrm{MHz}, \mathrm{CDCl}_{3}\right): \delta=7.43-7.35$ (comp, 2H), 7.30-7.22 (comp, 3H), 4.03 (dd, $\left.J=7.3,5.3 \mathrm{~Hz}, 1 \mathrm{H}\right)$, 3.20-3.09 (m, 1H), 2.92 (ddd, $J=10.3,7.9,5.5 \mathrm{~Hz}, 1 \mathrm{H}), 2.19-2.05$ (comp, 2H), 1.98-1.84 (comp, 2H), $1.83-1.71$ $(\mathrm{m}, 1 \mathrm{H})$.

${ }^{13}$ C-NMR $\left(125 \mathrm{MHz}, \mathrm{CDCl}_{3}\right): \delta=131.7,128.2,127.9,123.4,91.7,82.4,49.4,46.3,33.3,25.0$.

Accurate Mass (ESI): Calculated for $\mathrm{C}_{12} \mathrm{H}_{14} \mathrm{~N}[\mathrm{M}+\mathrm{H}]^{+}: 172.1121$, Found: 172.1018 , Spectral Accuracy: $98.5 \%$. 


\section{2-(Phenylethynyl)piperidine}

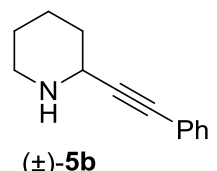

Following general procedures $\mathrm{A}$ and $\mathrm{C}$, compound $( \pm)-\mathbf{5 b}$ was obtained from piperidine $(99 \mu \mathrm{L}, 1 \mathrm{mmol})$, trifluoroacetophenone $(191.5 \mathrm{mg}, 1.1 \mathrm{mmol})$, ethynylbenzene $(153 \mathrm{mg}, 1.5 \mathrm{mmol})$, and $\mathrm{BF}_{3} \cdot \mathrm{OEt}_{2}(148 \mu \mathrm{L}, 1.2$ $\mathrm{mmol})$. Compound $( \pm)-5 \mathbf{b}$ was obtained as a light yellow oil in $60 \%$ yield $(111 \mathrm{mg})$.

\section{Characterization data:}

$\mathbf{R}_{\mathbf{f}}=0.24$ in $\mathrm{EtOAc} / \mathrm{MeOH} / i-\mathrm{PrNH}_{2}$ 90:9:1 v/v/v)

${ }^{1}$ H-NMR (500 MHz, $\mathrm{CDCl}_{3}$ ): $\delta=7.44-7.39$ (comp, 2H), 7.29-7.25 (comp, 3H), 3.81 (dd, J= 7.6, 3.5 Hz, $\left.1 \mathrm{H}\right), 3.12$ (app dt, $J=11.8,4.5 \mathrm{~Hz}, 1 \mathrm{H}), 2.72$ (ddd, $J=11.7,7.1,4.6 \mathrm{~Hz}, 1 \mathrm{H}), 1.95-1.77$ (comp, 3H), 1.68 (dddd, $J=12.6,8.6$, 7.5, $3.8 \mathrm{~Hz}, 1 \mathrm{H}), 1.59-1.35$ (comp, 3H).

${ }^{13}$ C-NMR $\left(125 \mathrm{MHz}, \mathrm{CDCl}_{3}\right): \delta=131.7,128.3,127.9,123.4,91.0,83.2,47.9,44.9,32.4,26.1,23.0$.

Accurate Mass (ESI): Calculated for $\mathrm{C}_{13} \mathrm{H}_{16} \mathrm{~N}[\mathrm{M}+\mathrm{H}]^{+}:$186.1272, Found: 186.1117, Spectral Accuracy: $98.8 \%$. 


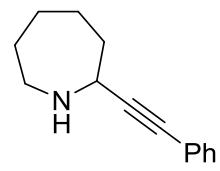

$( \pm)-5 c$

Following general procedures $\mathrm{A}$ and $\mathrm{C}$, compound $( \pm)-\mathbf{5 c}$ was obtained from azepane $(113 \mu \mathrm{L}, 1 \mathrm{mmol})$, trifluoroacetophenone $(191.5 \mathrm{mg}, 1.1 \mathrm{mmol})$, ethynylbenzene $(153 \mathrm{mg}, 1.5 \mathrm{mmol})$, and $\mathrm{BF}_{3} \cdot \mathrm{OEt}_{2}(148 \mu \mathrm{L}, 1.2$ mmol). Compound $( \pm)-\mathbf{5 c}$ was obtained as a colorless oil in $53 \%$ yield $(105.4 \mathrm{mg})$.

\section{Characterization data:}

$\mathbf{R}_{\mathbf{f}}=0.39$ in $\mathrm{EtOAc} / \mathrm{MeOH} / i-\mathrm{PrNH}_{2}$ 90:9:1 v/v/v)

${ }^{1}$ H-NMR $\left(500 \mathrm{MHz}, \mathrm{CDCl}_{3}\right.$ ): $\delta=7.32-7.27$ (comp, 2H), 7.20-7.10 (comp, 3H), 3.82 (dd, $\left.J=8.2,4.7 \mathrm{~Hz}, 1 \mathrm{H}\right), 2.99$ (ddd, $J=13.7,6.5,3.5 \mathrm{~Hz}, 1 \mathrm{H}), 2.70$ (ddd, $J=14.3,8.1,3.4 \mathrm{~Hz}, 1 \mathrm{H}), 2.45-2.09$ (m, 1H), 1.94 (dddd, $J=11.5,8.2$, $4.8,2.7 \mathrm{~Hz}, 1 \mathrm{H}), 1.82-1.65$ (comp, 3H), 1.62-1.59 (m, 1H), 1.57-1.44 (comp, 3H).

${ }^{13}$ C-NMR $\left(125 \mathrm{MHz}, \mathrm{CDCl}_{3}\right): \delta=131.7,128.2,127.9,123.4,91.9,82.9,50.4,45.9,37.0,30.8,27.2,24.9$.

Accurate Mass (ESI): Calculated for $\mathrm{C}_{14} \mathrm{H}_{18} \mathrm{~N}[\mathrm{M}+\mathrm{H}]^{+}:$200.1434, Found: 200.1330, Spectral Accuracy: 98.6\%. 


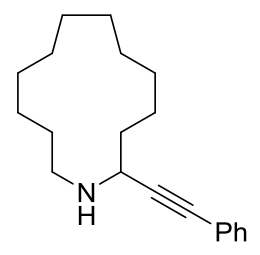

$( \pm)-5 d$

Following general procedures A and C, compound ( \pm )-5d was obtained from azacyclotridecane (183.2 $\mathrm{mg}, 1 \mathrm{mmol})$, trifluoroacetophenone $(191.5 \mathrm{mg}, 1.1 \mathrm{mmol})$, ethynylbenzene $(153 \mathrm{mg}, 1.5 \mathrm{mmol})$, and $\mathrm{BF}_{3} \cdot \mathrm{OEt}_{2}(148 \mu \mathrm{L}, 1.2$ $\mathrm{mmol})$. Compound $( \pm)$-5d was obtained as a colorless oil in $62 \%$ yield $(175.6 \mathrm{mg})$.

\section{Characterization data:}

$\mathbf{R}_{\mathbf{f}}=0.48$ in EtOAc/Hexanes 90:10 v/v/v)

${ }^{1} \mathbf{H}-\mathrm{NMR}\left(500 \mathrm{MHz}, \mathrm{CDCl}_{3}\right.$ ): $\delta=7.47-7.34$ (comp, 2H), 7.24-7.29 (comp, 3H), 3.59 (dd, $\left.J=9.4,3.8 \mathrm{~Hz}, 1 \mathrm{H}\right), 2.92$ (app td, $J=7.8,7.3,3.8 \mathrm{~Hz}, 1 \mathrm{H}), 2.80-2.65(\mathrm{~m}, 1 \mathrm{H}), 1.80$ (dd, $J=10.1,5.3 \mathrm{~Hz}, 1 \mathrm{H}), 1.71-1.64$ (comp, 3H), 1.60 1.52 (comp, 3H), 1.48-1.30 (comp, 14H).

${ }^{13}$ C-NMR $\left(125 \mathrm{MHz}, \mathrm{CDCl}_{3}\right): \delta=131.78$ 128.3, 127.8, 123.7, 92.4, 82.6, 49.1, 45.8, 35.0, 28.1, 26.8, 26.7, 26.2, 26.1, 25.5, 25.4, 24.8, 23.9.

HRMS (ESI-TOF): Calculated for $\mathrm{C}_{20} \mathrm{H}_{30} \mathrm{~N}[\mathrm{M}+\mathrm{H}]^{-}: 284.2373$, Found: 284.2375 . 


\section{1-Methyl-3-(phenylethynyl)piperazine}

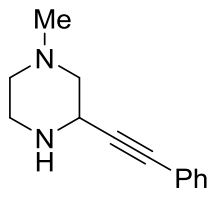

$( \pm)-5 e$

Following general procedures A and D, compound $( \pm)$-5e was obtained from 1-methylpiperazine $(111 \mu \mathrm{L}, 1 \mathrm{mmol})$, trifluoroacetophenone $(191.5 \mathrm{mg}, 1.1 \mathrm{mmol})$, ethynylbenzene $(153 \mathrm{mg}, 1.5 \mathrm{mmol})$, and $\mathrm{BF}_{3} \cdot \mathrm{OEt}_{2}(148 \mu \mathrm{L}, 1.2$ mmol). Compound $( \pm)$-5e was obtained as a white solid in $65 \%$ yield $(130.2 \mathrm{mg})$.

\section{Characterization data:}

$\mathbf{R}_{\mathbf{f}}=0.11$ in $\mathrm{EtOAc} / \mathrm{MeOH} / i-\mathrm{PrNH}_{2}$ 90:9:1 v/v/v)

${ }^{1}$ H-NMR (500 MHz, $\mathrm{CDCl}_{3}$ ): $\delta=7.48-7.34$ (comp, 2H), 7.29-7.17 (comp, 3H), 3.96-3.81 (m, $\left.1 \mathrm{H}\right), 3.06$ (app dt, $J$ $=12.5,3.4 \mathrm{~Hz}, 1 \mathrm{H}), 2.97-2.78(\mathrm{comp}, 2 \mathrm{H}), 2.68-2.50(\mathrm{~m}, 1 \mathrm{H}), 2.29(\mathrm{~s}, 3 \mathrm{H}), 2.27-2.11(\mathrm{comp}, 2 \mathrm{H}), 1.93(\mathrm{~s}, 1 \mathrm{H})$.

${ }^{13}$ C-NMR $\left(125 \mathrm{MHz}, \mathrm{CDCl}_{3}\right): \delta=131.8,128.3,128.2,123.0,88.9,83.3,60.8,55.4,47.6,46.4,44.9$.

Accurate Mass (ESI): Calculated for $\mathrm{C}_{13} \mathrm{H}_{17} \mathrm{~N}_{2}[\mathrm{M}+\mathrm{H}]^{+}:$201.1386, Found: 201.1298, Spectral Accuracy: $98.1 \%$. 


\section{2-((4-Methoxyphenyl)ethynyl)piperidine}

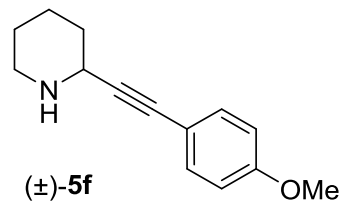

Following general procedures $\mathrm{A}$ and $\mathrm{C}$, compound $( \pm)$-5f was obtained from piperidine $(99 \mu \mathrm{L}, 1 \mathrm{mmol})$, trifluoroacetophenone $(191.5 \mathrm{mg}, 1.1 \mathrm{mmol})$, 1-ethynyl-4-methoxybenzene (198.1 $\mathrm{mg}, 1.5 \mathrm{mmol})$, and $^{\mathrm{BF}}{ }_{3} \bullet \mathrm{OEt}_{2}$ $(148 \mu \mathrm{L}, 1.2 \mathrm{mmol})$. Compound $( \pm)$-5f was obtained as a colorless oil in $50 \%$ yield $(107.5 \mathrm{mg})$.

\section{Characterization data:}

$\mathbf{R}_{\mathbf{f}}=0.25$ in $\mathrm{EtOAc} / \mathrm{MeOH} / i-\mathrm{PrNH}_{2}$ 90:9:1 v/v/v)

${ }^{1}$ H-NMR (500 MHz, $\left.\mathrm{CDCl}_{3}\right): \delta=7.35(\mathrm{~d}, J=8.4 \mathrm{~Hz}, 2 \mathrm{H}), 6.81$ (d, $\left.J=8.4 \mathrm{~Hz}, 2 \mathrm{H}\right), 3.83-3.75$ (comp, 4H), 3.16$3.06(\mathrm{~m}, 1 \mathrm{H}), 2.73$ (ddd, $J=11.8,7.3,4.2 \mathrm{~Hz}, 1 \mathrm{H}), 2.26$ (app s, 1H), 1.89 (app dq, $J=13.3,3.5 \mathrm{~Hz}, 1 \mathrm{H}), 1.82$ (ddd, $J=16.0,6.8,2.8 \mathrm{~Hz}, 1 \mathrm{H}), 1.68$ (app qd, $J=8.5,4.1 \mathrm{~Hz}, 1 \mathrm{H}), 1.60-1.37$ (comp, 3H).

${ }^{13}$ C-NMR $\left(125 \mathrm{MHz}, \mathrm{CDCl}_{3}\right): \delta=159.4,133.1,115.5,113.9,89.3,83.1,55.4,47.9,44.9,32.5,26.0,23.0$.

Accurate Mass (ESI): Calculated for $\mathrm{C}_{14} \mathrm{H}_{18} \mathrm{NO}[\mathrm{M}+\mathrm{H}]^{+}: 216.1383$, Found: 216.1325 , Spectral Accuracy: $98.7 \%$. 


\section{2-((3-Methoxyphenyl)ethynyl)piperidine}

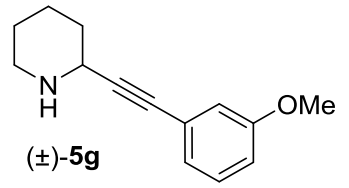

Following general procedures $\mathrm{A}$ and $\mathrm{C}$, compound $( \pm)-5 \mathrm{~g}$ was obtained from piperidine $(99 \mu \mathrm{L}, 1 \mathrm{mmol})$, trifluoroacetophenone $(191.5 \mathrm{mg}, 1.1 \mathrm{mmol})$, 1-ethynyl-3-methoxybenzene (198.1 mg, $1.5 \mathrm{mmol})$, and $\mathrm{BF}_{3} \cdot \mathrm{OEt}_{2}$ $(148 \mu \mathrm{L}, 1.2 \mathrm{mmol})$. Compound $( \pm)-5 \mathrm{~g}$ was obtained as a colorless oil in $57 \%$ yield $(122.6 \mathrm{mg})$.

\section{Characterization data:}

$\mathbf{R}_{\mathbf{f}}=0.36$ in $\mathrm{EtOAc} / \mathrm{MeOH} / i-\mathrm{PrNH}_{2}$ 90:9:1 v/v/v)

${ }^{1}$ H-NMR $\left(500 \mathrm{MHz}, \mathrm{CDCl}_{3}\right): \delta=7.18($ app t, $J=7.9 \mathrm{~Hz}, 1 \mathrm{H}), 7.01(\mathrm{~d}, J=7.5 \mathrm{~Hz}, 1 \mathrm{H}), 6.96-6.93(\mathrm{~m}, 1 \mathrm{H}), 6.86-$ $6.81(\mathrm{~m}, 1 \mathrm{H}), 3.81(\mathrm{dd}, J=7.8,3.5 \mathrm{~Hz}, 1 \mathrm{H}), 3.78(\mathrm{~s}, 3 \mathrm{H}), 3.12(\mathrm{app} \mathrm{dt}, J=11.2,5.0 \mathrm{~Hz}, 1 \mathrm{H}), 2.84-2.68(\mathrm{~m}, 1 \mathrm{H})$, 2.00-1.86 (comp, 2H), 1.84-1.79 (m, 1H), 1.69 (app qd, $J=8.3,4.0 \mathrm{~Hz}, 1 \mathrm{H}), 1.59-1.45$ (comp, 3H).

${ }^{13}$ C-NMR (125 MHz, $\left.\mathrm{CDCl}_{3}\right): \delta=159.4,129.4,124.4,124.3,116.6,114.7,90.8,83.1,55.3,47.9,44.9,32.4,26.0$, 23.0.

Accurate Mass (ESI): Calculated for $\mathrm{C}_{14} \mathrm{H}_{18} \mathrm{NO}[\mathrm{M}+\mathrm{H}]^{+}: 216.1383$, Found: 216.1288 , Spectral Accuracy: 98.7\%. 


\section{2-(Cyclohex-1-en-1-ylethynyl)piperidine}

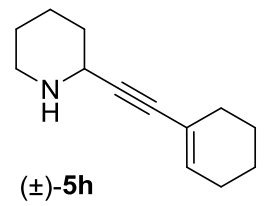

Following general procedures $\mathrm{A}$ and $\mathrm{C}$, compound $( \pm)-\mathbf{5 h}$ was obtained from piperidine $(99 \mu \mathrm{L}, 1 \mathrm{mmol})$, trifluoroacetophenone $(191.5 \mathrm{mg}, 1.1 \mathrm{mmol})$, 1-ethynylcyclohex-1-ene $(159.2 \mathrm{mg}, 1.5 \mathrm{mmol})$, and $\mathrm{BF}_{3} \bullet \mathrm{OEt}_{2}(148$ $\mu \mathrm{L}, 1.2 \mathrm{mmol})$. Compound $( \pm)-5 \mathrm{~h}$ was obtained as a colorless oil in $60 \%$ yield $(113.4 \mathrm{mg})$.

\section{Characterization data:}

$\mathbf{R}_{\mathbf{f}}=0.34$ in EtOAc/MeOH$/ i-\mathrm{PrNH}_{2}$ 90:9:1 v/v/v)

${ }^{1}$ H-NMR (500 MHz, $\mathrm{CDCl}_{3}$ ): $\delta=6.04(\operatorname{app~td}, J=4.0,2.0 \mathrm{~Hz}, 1 \mathrm{H}), 3.69(\mathrm{dd}, J=7.8,3.3 \mathrm{~Hz}, 1 \mathrm{H}), 3.10-3.01(\mathrm{~m}$, 1H), 2.67 (ddd, $J=11.7,7.3,4.3 \mathrm{~Hz}, 1 \mathrm{H}$ ), 2.14-1.99 (comp, 4H), 1.92-1.85 (m, 1H), 1.84-1.69 (comp, 2H), 1.651.38 (comp, 8H).

${ }^{13}$ C-NMR $\left(125 \mathrm{MHz}, \mathrm{CDCl}_{3}\right): \delta=134.3,120.7,88.2,84.9,47.9,44.9,32.6,29.6,26.1,25.7,23.0,22.5,21.7$.

Accurate Mass (ESI): Calculated for $\mathrm{C}_{13} \mathrm{H}_{20} \mathrm{~N}[\mathrm{M}+\mathrm{H}]^{+}$: 190.1590, Found: 190.1521, Spectral Accuracy: 98.4\%. 


\section{2-(4-Phenylbut-1-yn-1-yl)pyrrolidine}

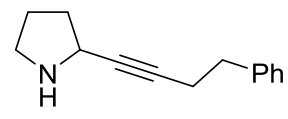

$( \pm)-5 \mathbf{i}$

Following general procedures $\mathrm{A}$ and $\mathrm{C}$, compound $( \pm)-\mathbf{5 i}$ was obtained from pyrrolidine $(82 \mu \mathrm{L}, 1 \mathrm{mmol})$, benzophenone (200.2 mg, $1.1 \mathrm{mmol})$, but-3-yn-1-ylbenzene (195.2 mg, $1.5 \mathrm{mmol})$, and $\mathrm{BF}_{3} \cdot \mathrm{OEt}_{2}(148 \mu \mathrm{L}, 1.2$ $\mathrm{mmol})$. Compound $( \pm)-5 \mathbf{i}$ was obtained as a colorless oil in $55 \%$ yield $(109.5 \mathrm{mg})$.

\section{Characterization data:}

$\mathbf{R}_{\mathbf{f}}=0.19$ in $\mathrm{EtOAc} / \mathrm{MeOH} / i-\mathrm{PrNH}_{2}$ 90:9:1 v/v)

${ }^{1} \mathrm{H}-\mathrm{NMR}\left(500 \mathrm{MHz}, \mathrm{CDCl}_{3}\right): \delta=7.36-7.29$ (comp, 2H), 7.28-7.22 (comp, 3H), 3.82 (app td, $J=6.4,5.1,2.1 \mathrm{~Hz}$, 1H), 3.18-3.05 (m, 1H), 2.96-2.87 (m, 1H), 2.87-2.82 (comp 2H), 2.54-2.48 (comp, 2H), 2.11-1.99 (comp, 2H), $1.94-1.83(\mathrm{~m}, 1 \mathrm{H}), 1.83-1.70$ (comp, 2H).

${ }^{13}$ C-NMR $\left(125 \mathrm{MHz}, \mathrm{CDCl}_{3}\right): \delta=140.9,128.6,128.4,126.3,83.1,82.0,49.1,46.2,35.4,33.5,25.0,21.1$.

Accurate Mass (ESI): Calculated for $\mathrm{C}_{14} \mathrm{H}_{18} \mathrm{~N}[\mathrm{M}+\mathrm{H}]^{+}:$200.1434, Found: 200.1275, Spectral Accuracy: 99.1\%. 
2-(3-(Benzyloxy)prop-1-yn-1-yl)piperidine

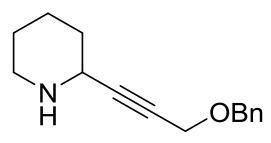

$( \pm)-5$

Following general procedures $\mathrm{A}$ and $\mathrm{C}$, compound $( \pm)-5 \mathbf{j}$ was obtained from piperidine $(99 \mu \mathrm{L}, 1 \mathrm{mmol})$, trifluoroacetophenone $(191.5 \mathrm{mg}, 1.1 \mathrm{mmol})$, ((prop-2-yn-1-yloxy)methyl)benzene $(219.2 \mathrm{mg}, 1.5 \mathrm{mmol})$, and $\mathrm{BF}_{3} \cdot \mathrm{OEt}_{2}(148 \mu \mathrm{L}, 1.2 \mathrm{mmol})$. Compound $( \pm)-5 \mathbf{j}$ was obtained as a colorless oil in $52 \%$ yield $(119.2 \mathrm{mg})$.

\section{Characterization data:}

$\mathbf{R}_{\mathbf{f}}=0.24$ in EtOAc/MeOH/i-PrNH $\left.290: 9: 1 \mathrm{v} / \mathrm{v} / \mathrm{v}\right)$

${ }^{1}$ H-NMR $\left(500 \mathrm{MHz}, \mathrm{CDCl}_{3}\right): \delta=7.38-7.33$ (comp, 4H), 7.32-7.27 (m, 1H), 4.59 (s, 2H), 4.20 (d, J = 1.8 Hz, 2H), $3.68(\mathrm{ddd}, J=7.5,3.5,1.7 \mathrm{~Hz}, 1 \mathrm{H}), 3.17-2.96(\mathrm{~m}, 1 \mathrm{H}), 2.70(\mathrm{ddd}, J=11.8,7.0,4.7 \mathrm{~Hz}, 1 \mathrm{H}), 2.06(\mathrm{~s}, 1 \mathrm{H}), 1.94-$ 1.71 (comp, 2H), 1.69-1.39 (comp, 4H).

${ }^{13}$ C-NMR $\left(125 \mathrm{MHz}, \mathrm{CDCl}_{3}\right): \delta=137.7,128.5,128.2,127.9,88.0,78.9,71.6,57.7,47.4,44.7,32.2,25.9,22.9$.

Accurate Mass (ESI): Calculated for $\mathrm{C}_{15} \mathrm{H}_{20} \mathrm{NO}[\mathrm{M}+\mathrm{H}]^{+}: 230.1539$, Found: 230.1506 , Spectral Accuracy: 99.4\%. 
trans-4-Methyl-2-(phenylethynyl)piperidine

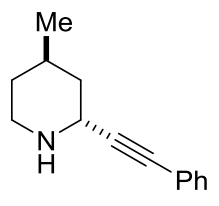

$( \pm)-5 k$

Following general procedures A and D, compound ( \pm )-5k was obtained from 4-methylpiperidine $(118 \mu \mathrm{L}, 1 \mathrm{mmol})$, trifluoroacetophenone (191.5 mg, $1.1 \mathrm{mmol})$, ethynylbenzene $(153 \mathrm{mg}, 1.5 \mathrm{mmol})$, and $\mathrm{BF}_{3} \cdot \mathrm{OEt}_{2}(148 \mu \mathrm{L}, 1.2$ $\mathrm{mmol})$. Compound $( \pm)$-5k was obtained as a colorless oil in $55 \%$ yield $(109.5 \mathrm{mg})$.

\section{Characterization data:}

$\mathbf{R}_{\mathbf{f}}=0.27$ in EtOAc/MeOH$/ i-\mathrm{PrNH}_{2}$ 90:9:1 v/v/v)

${ }^{1}$ H-NMR (500 MHz, $\mathrm{CDCl}_{3}$ ): $\delta=7.46-7.38$ (comp, 2H), 7.31-7.26 (comp, 3H), 4.16 (app t, J=3.7 Hz, 1H), 3.32$3.05(\mathrm{~m}, 1 \mathrm{H}), 2.86(\mathrm{app} \mathrm{dt}, J=11.4,3.6 \mathrm{~Hz}, 1 \mathrm{H}), 1.95$ (app dtd, $J=11.2,6.9,3.5 \mathrm{~Hz}, 1 \mathrm{H}), 1.88-1.76(\mathrm{comp}, 2 \mathrm{H})$, 1.65 (app dt, $J=13.1,2.9 \mathrm{~Hz}, 1 \mathrm{H}), 1.45$ (app td, $J=12.3,4.6 \mathrm{~Hz}, 1 \mathrm{H}), 1.17$ (app qd, $J=12.1,4.2 \mathrm{~Hz}, 1 \mathrm{H}), 0.94(\mathrm{~d}$, $J=6.6 \mathrm{~Hz}, 3 \mathrm{H})$.

${ }^{13}$ C-NMR $\left(125 \mathrm{MHz}, \mathrm{CDCl}_{3}\right): \delta=131.7,128.3,127.9,123.5,91.1,84.0,46.1,41.7,39.2,34.7,26.9,22.2$.

Accurate Mass (ESI): Calculated for $\mathrm{C}_{14} \mathrm{H}_{18} \mathrm{~N}[\mathrm{M}+\mathrm{H}]^{+}: 200.1434$, Found: 200.1322, Spectral Accuracy: $98.7 \%$. 


\section{1-(Phenylethynyl)octahydrocyclopenta[c]pyrrole}

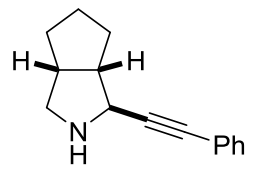

$( \pm)-5$ I

Following general procedures $\mathrm{A}$ and $\mathrm{D}$, compound $( \pm)$-5I was obtained from octahydrocyclopenta[c]pyrrole (111.1 $\mathrm{mg}, 1 \mathrm{mmol})$ trifluoroacetophenone $(191.5 \mathrm{mg}, 1.1 \mathrm{mmol})$, ethynylbenzene $(153 \mathrm{mg}, 1.5 \mathrm{mmol})$, and $\mathrm{BF}_{3} \cdot \mathrm{OEt}_{2}(148$ $\mu \mathrm{L}, 1.2 \mathrm{mmol})$. Compound $( \pm)-5 \mathbf{l}$ was obtained as a colorless oil in $65 \%$ yield $(137.1 \mathrm{mg})$.

\section{Characterization data:}

$\mathbf{R}_{\mathbf{f}}=0.62$ in EtOAc/MeOH$/ i-\mathrm{PrNH}_{2}$ 90:9:1 v/v/v)

${ }^{1}$ H-NMR (500 MHz, $\mathrm{CDCl}_{3}$ ): $\delta=7.43-7.37$ (comp, 2H), 7.29-7.23 (comp, 3H), 3.57 (d, $\left.J=4.3 \mathrm{~Hz}, 1 \mathrm{H}\right), 3.40-3.29$ (m, 1H), 2 73-2.60 (comp, 2H), 2.52 (dd, $J=10.5,4.5 \mathrm{~Hz}, 1 \mathrm{H}$ ), 2.18 (s, 1H), 1.77 (app tt, $J=8.1,5.7 \mathrm{~Hz}, 1 \mathrm{H}$ ), 1.69 (ddd, $J=14.4,9.5,6.1 \mathrm{~Hz}, 1 \mathrm{H}), 1.63-1.46$ (comp, 3H), 1.37 (app dtd, $J=11.5,5.6,2.9 \mathrm{~Hz}, 1 \mathrm{H})$.

${ }^{13} \mathbf{C}-\mathrm{NMR}\left(125 \mathrm{MHz}, \mathrm{CDCl}_{3}\right): \delta=131.7,128.3,127.9,123.4,91.2,82.7,56.6,53.5,52.3,43.7,32.7,32.0,26.0$.

Accurate Mass (ESI): Calculated for $\mathrm{C}_{15} \mathrm{H}_{18} \mathrm{~N}[\mathrm{M}+\mathrm{H}]^{+}: 212.1434$, Found: 212.1313, Spectral Accuracy: 98.7\%. 
2-(Furan-2-yl)pyrrolidine

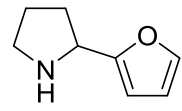

(土)-6a

Following general procedure A, compound $( \pm)$-6a was obtained from pyrrolidine ( $82 \mu \mathrm{L}, 1 \mathrm{mmol})$, benzophenone $(219 \mathrm{mg}, 1.2 \mathrm{mmol})$, the organometallic nucleophile $(1.5 \mathrm{mmol})$, and $\mathrm{BF}_{3} \cdot \mathrm{OEt}_{2}(148 \mu \mathrm{L}, 1.2 \mathrm{mmol})$. Compound $( \pm)$-6a was obtained as a yellow oil in $42 \%$ yield $(57.6 \mathrm{mg})$.

The organometallic nucleophile was prepared as follows: To a solution of furan $(109 \mu \mathrm{L}, 1.5 \mathrm{mmol})$ in anhydrous THF ( $2 \mathrm{~mL})$, cooled to $-78{ }^{\circ} \mathrm{C}$ was slowly added $n$-BuLi in hexanes ( $1.5 \mathrm{mmol}, 1.5$ equiv to the amine) under the protection of nitrogen. The resulting mixture was stirred at the same temperature for $10 \mathrm{~min}$ and then warmed to $0{ }^{\circ} \mathrm{C}$ over $30 \mathrm{~min}$, to give the corresponding lithiated furan solution.

\section{Characterization data:}

$\mathbf{R}_{\mathbf{f}}=0.24$ in $\mathrm{EtOAc} / \mathrm{MeOH} / i-\mathrm{PrNH}_{2}$ 90:9:1 v/v/v)

${ }^{1}$ H-NMR $\left(400 \mathrm{MHz}, \mathrm{CDCl}_{3}\right): \delta=7.32(\mathrm{dd}, J=1.9,0.8 \mathrm{~Hz}, 1 \mathrm{H}), 6.27(\mathrm{dd}, J=3.2,1.8 \mathrm{~Hz}, 1 \mathrm{H}), 6.12(\operatorname{app~dt}, J=3.2$, $0.8 \mathrm{~Hz}, 1 \mathrm{H}), 4.27-4.08(\mathrm{~m}, 1 \mathrm{H}), 3.19-3.01(\mathrm{~m}, 1 \mathrm{H}), 2.99-2.88(\mathrm{~m}, 1 \mathrm{H}), 2.18-2.00$ (comp, 2H), 1.96-1.72 (comp, $3 \mathrm{H})$.

${ }^{13}$ C-NMR $\left(100 \mathrm{MHz}, \mathrm{CDCl}_{3}\right): \delta=157.5,141.6,110.1,104.9,55.9,46.7,30.9,25.4$.

Accurate Mass (ESI): Calculated for $\mathrm{C}_{8} \mathrm{H}_{12} \mathrm{NO}[\mathrm{M}+\mathrm{H}]^{+}:$138.0913, Found: 138.0944, Spectral Accuracy: 98.3\%. 


\section{2-(Benzofuran-2-yl)pyrrolidine}

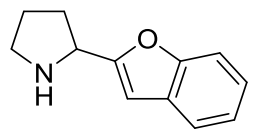

(土)-6b

Following general procedure A, compound $( \pm)$-6b was obtained from pyrrolidine $(82 \mu \mathrm{L}, 1 \mathrm{mmol})$, benzophenone (219 $\mathrm{mg}, 1.2 \mathrm{mmol})$, the organometallic nucleophile $(1.5 \mathrm{mmol})$, and $\mathrm{BF}_{3} \cdot \mathrm{OEt}_{2}(148 \mu \mathrm{L}, 1.2 \mathrm{mmol})$. Compound $( \pm)$-6b was obtained as a yellow oil in $62 \%$ yield $(116 \mathrm{mg})$.

The organometallic nucleophile was prepared as follows: To a solution of the benzofuran $(165 \mu \mathrm{L}, 1.5 \mathrm{mmol})$ in anhydrous THF $(2 \mathrm{~mL})$, cooled to $-78{ }^{\circ} \mathrm{C}$ was slowly added $n$-BuLi in hexanes (1.5 mmol, 1.5 equiv) under the protection of nitrogen. The resulting mixture was stirred at the same temperature for $30 \mathrm{~min}$, to give the corresponding lithiated benzofuran solution.

\section{Characterization data:}

$\mathbf{R}_{\mathbf{f}}=0.27$ in EtOAc/MeOH/i-PrNH $\left.290: 9: 1 \mathrm{v} / \mathrm{v} / \mathrm{v}\right)$

${ }^{1} \mathbf{H}-\mathbf{N M R}\left(400 \mathrm{MHz}, \mathrm{CDCl}_{3}\right): \delta=7.54-7.46(\mathrm{~m}, 1 \mathrm{H}), 7.43(\mathrm{dd}, J=8.2,0.9 \mathrm{~Hz}, 1 \mathrm{H}), 7.25-7.11(\mathrm{comp}, 2 \mathrm{H}), 6.53(\mathrm{~s}$, $1 \mathrm{H}), 4.34$ (ddd, $J=7.4,6.3,0.8 \mathrm{~Hz}, 1 \mathrm{H}), 3.15$ (ddd, $J=10.3,7.6,5.6 \mathrm{~Hz}, 1 \mathrm{H}), 3.01$ (ddd, $J=10.2,7.9,6.1 \mathrm{~Hz}, 1 \mathrm{H}$ ), 2.30-2.09 (comp, 2H), 2.08-1.90 (comp, 2H), 1.90-1.76 (m, 1H).

${ }^{13}$ C-NMR $\left(100 \mathrm{MHz}, \mathrm{CDCl}_{3}\right): \delta=160.6,154.9,128.6,123.7,122.6,120.7,111.2,101.9,56.2,46.9,31.1,25.5$.

Accurate Mass (ESI): Calculated for $\mathrm{C}_{12} \mathrm{H}_{14} \mathrm{NO}[\mathrm{M}+\mathrm{H}]^{+}$: 188.1070, Found: 188.0914, Spectral Accuracy: 98.2\%. 


\section{1-Methyl-2-(pyrrolidin-2-yl)-1H-indole}

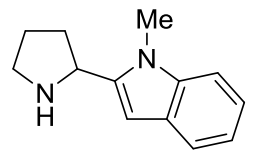

$( \pm)-6 c$

Following the general procedure E, compound $( \pm)-6 \mathbf{c}$ was obtained from pyrrolidine $(82 \mu \mathrm{L}, 1 \mathrm{mmol})$ and benzophenone (219 mg, $1.2 \mathrm{mmol})$. Compound $( \pm)-6 \mathbf{c}$ was obtained as a colorless oil in 51\% (102 mg).

\section{Characterization data:}

$\mathbf{R}_{\mathbf{f}}=0.16$ in $\left.\mathrm{EtOAc} / \mathrm{MeOH} / i-\mathrm{PrNH}_{2} 90: 9: 1 \mathrm{v} / \mathrm{v} / \mathrm{v}\right)$

${ }^{1}$ H-NMR (400 MHz, $\left.\mathrm{CDCl}_{3}\right): \delta=7.59(\mathrm{ddd}, J=7.9,2.3,1.2 \mathrm{~Hz}, 1 \mathrm{H}), 7.32(\mathrm{~d}, J=8.1 \mathrm{~Hz}, 1 \mathrm{H}), 7.22(\operatorname{app~ddt}, J=$ 8.3, 6.9, $1.4 \mathrm{~Hz}, 1 \mathrm{H}), 7.12$ (app ddt, $J=9.4,7.2,1.3 \mathrm{~Hz}, 1 \mathrm{H}), 6.43(\mathrm{~d}, J=0.9 \mathrm{~Hz}, 1 \mathrm{H}), 4.30(\operatorname{app~t}, J=6.8 \mathrm{~Hz}, 1 \mathrm{H})$, $3.80(\mathrm{~d}, J=1.0 \mathrm{~Hz}, 3 \mathrm{H}), 3.31-3.14(\mathrm{~m}, 1 \mathrm{H}), 3.12-2.88(\mathrm{~m}, 1 \mathrm{H}), 2.24$ (app ddt, $J=9.6,6.7,3.0 \mathrm{~Hz}, 1 \mathrm{H}), 2.15-1.81$ (comp, 4H).

${ }^{13}$ C-NMR $\left(100 \mathrm{MHz}, \mathrm{CDCl}_{3}\right): \delta=142.7,137.9,127.4,121.2,120.3,119.4,108.9,97.8,55.5,47.1,31.8,30.1,25.7$. Accurate Mass (ESI): Calculated for $\mathrm{C}_{13} \mathrm{H}_{17} \mathrm{~N}_{2}[\mathrm{M}+\mathrm{H}]^{+}:$201.1386, Found: 201.1291, Spectral Accuracy: 98.8\%. 


\section{1-(Phenylsulfonyl)-2-(pyrrolidin-2-yl)-1H-indole}

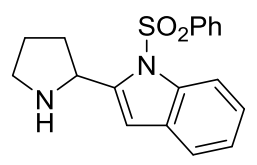

$( \pm)-6 d$

Following general procedure A, compound ( \pm )-6d was obtained from pyrrolidine ( $82 \mu \mathrm{L}, 1 \mathrm{mmol})$, benzophenone (219 $\mathrm{mg}, 1.2 \mathrm{mmol})$, the organometallic nucleophile $(1.5 \mathrm{mmol})$, and $\mathrm{BF}_{3} \cdot \mathrm{OEt}_{2}(148 \mu \mathrm{L}, 1.2 \mathrm{mmol})$. Compound $( \pm)$-6d was obtained as a colorless oil in $54 \%$ yield $(176 \mathrm{mg})$.

The organometallic nucleophile was prepared as follows: To a solution of diisopropylamine ( $211 \mu \mathrm{L}, 1.5 \mathrm{mmol}, 1.5$ equiv) in anhydrous THF $(1 \mathrm{~mL})$ cooled to $-78^{\circ} \mathrm{C}$ was slowly added $n$-BuLi in hexanes $(1.5 \mathrm{mmol}, 1.5$ equiv) under the protection of nitrogen. The resulting mixture was stirred at the same temperature for $20 \mathrm{~min}$. In a separate flame dried vial a solution of 1-(phenylsulfonyl)-1H-indole (385.5 mg, $1.5 \mathrm{mmol})$ in anhydrous THF $(1 \mathrm{~mL})$ was taken and cooled to $-78^{\circ} \mathrm{C}$ and to this was then added dropwise the solution of freshly prepared lithium diisopropyl amide and the mixture was stirred at $-78{ }^{\circ} \mathrm{C}$ for $10 \mathrm{~min}$ before being cooled in an ice bath for $20 \mathrm{~min}$ for the formation of the corresponding lithiated indole.

\section{Characterization data:}

$\mathbf{R}_{\mathbf{f}}=0.36$ in $\mathrm{EtOAc} / \mathrm{MeOH} / i-\mathrm{PrNH}_{2}$ 90:9:1 v/v/v)

${ }^{1}$ H-NMR $\left(400 \mathrm{MHz}, \mathrm{CDCl}_{3}\right.$ ): $\delta=7.81$ (app dt, $\left.J=8.3,0.9 \mathrm{~Hz}, 1 \mathrm{H}\right), 7.56-7.40$ (comp, 2H), 7.22-7.15 (m, 1H), 7.13 (dd, $J=7.4,1.0 \mathrm{~Hz}, 1 \mathrm{H}), 7.11-7.04$ (comp, 2H), 6.98-6.93 (m, 1H), 6.93-6.87 (m, 1H), 6.39 (s, 1H), 4.54 (app ddt, $J=7.7,6.4,1.1 \mathrm{~Hz}, 1 \mathrm{H}), 2.99-2.71$ (comp, 2H), 2.27-2.12 (m, 1H), 2.12-1.90 (m, 1H), 1.81-1.46 (comp, 3H).

${ }^{13}$ C-NMR $\left(100 \mathrm{MHz}, \mathrm{CDCl}_{3}\right): \delta=145.8,138.9,137.6,133.7,129.7,129.2,126.4,124.3,123.8,120.7,114.9,108.4$, $55.9,46.5,32.9,25.2$.

Accurate Mass (ESI): Calculated for $\mathrm{C}_{18} \mathrm{H}_{19} \mathrm{~N}_{2} \mathrm{O}_{2} \mathrm{~S}[\mathrm{M}+\mathrm{H}]^{+}:$327.1162, Found: 327.1206, Spectral Accuracy: $99.2 \%$. 


\section{3-Bromo-1-(phenylsulfonyl)-2-(pyrrolidin-2-yl)-1H-indole}

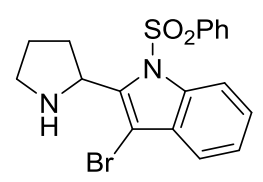

$( \pm)-6 e$

Following general procedure A, compound $( \pm)$-6e was obtained from pyrrolidine ( $82 \mu \mathrm{L}, 1 \mathrm{mmol})$, benzophenone (219 $\mathrm{mg}, 1.2 \mathrm{mmol})$, the organometallic nucleophile $(1.5 \mathrm{mmol})$, and $\mathrm{BF}_{3} \cdot \mathrm{OEt}_{2}(148 \mu \mathrm{L}, 1.2 \mathrm{mmol})$. Compound $( \pm)$-6e was obtained as a colorless oil in $59 \%$ yield $(238.3 \mathrm{mg})$.

The organometallic nucleophile was prepared as follows: To a solution of diisopropylamine ( $211 \mu \mathrm{L}, 1.5 \mathrm{mmol}, 1.5$ equiv) in anhydrous THF $(1 \mathrm{~mL})$ cooled to $-78^{\circ} \mathrm{C}$ was slowly added $n$-BuLi in hexanes $(1.5 \mathrm{mmol}, 1.5$ equiv) under the protection of nitrogen. The resulting mixture was stirred at the same temperature for $20 \mathrm{~min}$. In a separate flame dried vial a solution of 3-bromo-1-(phenylsulfonyl)-1H-indole (504.3 mg, $1.5 \mathrm{mmol}, 1.5$ equiv) in anhydrous THF $(1 \mathrm{~mL})$ was taken and cooled to $-78{ }^{\circ} \mathrm{C}$ and to this was then added dropwise the solution of freshly prepared lithium diisopropyl amide and the mixture was stirred at $-78^{\circ} \mathrm{C}$ for $60 \mathrm{~min}$ for the formation of the corresponding lithiated indole.

\section{Characterization data:}

$\mathbf{R}_{\mathbf{f}}=0.58$ in $\mathrm{EtOAc} / \mathrm{MeOH} / i-\mathrm{PrNH}_{2}$ 90:9:1 v/v/v)

${ }^{1}$ H-NMR $\left(400 \mathrm{MHz}, \mathrm{CDCl}_{3}\right): \delta=8.20$ (app dt, $\left.J=8.4,0.9 \mathrm{~Hz}, 1 \mathrm{H}\right), 7.84-7.75(\mathrm{comp}, 2 \mathrm{H}), 7.54-7.49$ (m, 1H), 7.47 (ddd, $J=7.7,1.5,0.7 \mathrm{~Hz}, 1 \mathrm{H}), 7.43-7.38$ (comp, 2H), 7.35 (ddd, $J=8.5,7.2,1.5 \mathrm{~Hz}, 1 \mathrm{H}), 7.32-7.28(\mathrm{~m}, 1 \mathrm{H}), 5.09$ (app t, $J=8.2 \mathrm{~Hz}, 1 \mathrm{H}), 3.32$ (ddd, $J=10.5,7.1,5.6 \mathrm{~Hz}, 1 \mathrm{H}), 2.97$ (ddd, $J=10.5,7.6,6.5 \mathrm{~Hz}, 1 \mathrm{H}), 2.37$ (s, $1 \mathrm{H}$ ), 2.26-2.14 (m, 1H), 2.11-1.99 (comp, 2H), 1.94-1.79 (m, 1H).

${ }^{13}$ C-NMR $\left(100 \mathrm{MHz}, \mathrm{CDCl}_{3}\right): \delta=139.1,138.7,136.1,134.0,129.8,129.4,126.5,125.9,124.4,119.5,115.5,100.9$, $55.3,48.1,32.0,27.1$.

Accurate Mass (ESI): Calculated for $\mathrm{C}_{18} \mathrm{H}_{18} \mathrm{BrN}_{2} \mathrm{O}_{2} \mathrm{~S}[\mathrm{M}+\mathrm{H}]^{+}$: 405.0267, Found: 405.0387, 407.0300, Spectral Accuracy: $98.6 \%$. 


\section{1-Methyl-2-(piperidin-2-yl)-1H-indole}

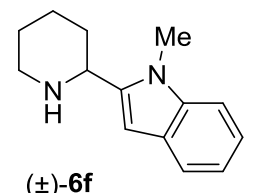

Following the general procedure E, compound ( \pm )-6f was obtained from piperidine $(99 \mu \mathrm{L}, 1 \mathrm{mmol})$, and trifluoroacetophenone $(191.5 \mathrm{mg}, 1.1 \mathrm{mmol})$. Compound ( \pm )-6f was obtained as a colorless oil in $46 \%$ (98.5 mg).

\section{Characterization data:}

$\mathbf{R}_{\mathbf{f}}=0.57$ in $\mathrm{EtOAc} / \mathrm{MeOH} / i-\mathrm{PrNH}_{2}$ 90:9:1 v/v/v)

${ }^{1}$ H-NMR $\left(500 \mathrm{MHz}, \mathrm{CDCl}_{3}\right.$ ): $\delta=7.59$ (app dt, $\left.J=8.0,0.9 \mathrm{~Hz}, 1 \mathrm{H}\right), 7.30$ (app dt, $J=8.3,0.9 \mathrm{~Hz}, 1 \mathrm{H}$ ), 7.20 (ddd, $J$ $=8.2,7.0,1.2 \mathrm{~Hz}, 1 \mathrm{H}), 7.10(\mathrm{ddd}, J=8.0,7.1,1.0 \mathrm{~Hz}, 1 \mathrm{H}), 6.43(\mathrm{~s}, 1 \mathrm{H}), 3.84-3.79(\mathrm{~m}, 1 \mathrm{H}), 3.77(\mathrm{~s}, 3 \mathrm{H}), 3.20(\operatorname{app}$ ddt, $J=12.3,3.9,1.9 \mathrm{~Hz}, 1 \mathrm{H}$ ), 2.81 (app td, $J=12.1,2.8 \mathrm{~Hz}, 1 \mathrm{H}$ ), 2.03-1.95 (comp, 2H), 1.86-1.41 (comp, 5H).

${ }^{13}$ C-NMR $\left(125 \mathrm{MHz}, \mathrm{CDCl}_{3}\right): \delta=143.5,137.7,127.6,121.1,120.3,119.1,108.9,98.1,54.3,47.5,32.6,29.9,26.6$, 25.2 .

Accurate Mass (ESI): Calculated for $\mathrm{C}_{14} \mathrm{H}_{19} \mathrm{~N}_{2}[\mathrm{M}+\mathrm{H}]^{+}: 215.1543$, Found: 215.1425, Spectral Accuracy: 98.1\%. 


\section{2-(Benzofuran-2-yl)piperidine}

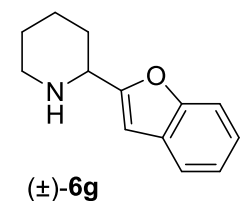

Following general procedure A, compound $( \pm)-6 \mathrm{~g}$ was obtained from piperidine $(99 \mu \mathrm{L}, 1 \mathrm{mmol})$, trifluoroacetophenone $(191.5 \mathrm{mg}, 1.1 \mathrm{mmol})$, the organometallic nucleophile $(1.5 \mathrm{mmol})$, and $\mathrm{BF}_{3} \cdot \mathrm{OEt}_{2}(148 \mu \mathrm{L}, 1.2$ mmol). Compound $( \pm)-\mathbf{6 g}$ was obtained as a yellow oil in $42 \%$ yield $(84.4 \mathrm{mg})$.

The organometallic nucleophile was prepared as follows: To a solution of the benzofuran ( $165 \mu \mathrm{L}, 1.5 \mathrm{mmol})$ in anhydrous THF $(2 \mathrm{~mL})$ cooled to $-78{ }^{\circ} \mathrm{C}$ was slowly added $n$-BuLi in hexanes (1.5 mmol, 1.5 equiv) under the protection of nitrogen. The resulting mixture was stirred at the same temperature for $30 \mathrm{~min}$, to give the corresponding lithiated benzofuran solution.

\section{Characterization data:}

$\mathbf{R}_{\mathbf{f}}=0.48$ in $\mathrm{EtOAc} / \mathrm{MeOH} / i-\mathrm{PrNH}_{2}$ 90:9:1 $\left.\mathrm{v} / \mathrm{v} / \mathrm{v}\right)$

${ }^{1} \mathbf{H}$-NMR (500 MHz, $\left.\mathrm{CDCl}_{3}\right): \delta=7.55-7.47(\mathrm{~m}, 1 \mathrm{H}), 7.47-7.39(\mathrm{~m}, 1 \mathrm{H}), 7.29-7.08(\mathrm{comp}, 2 \mathrm{H}), 6.51(\mathrm{~s}, 1 \mathrm{H}), 3.84$ (ddd, $J=10.6,3.1,0.9 \mathrm{~Hz}, 1 \mathrm{H}), 3.27-3.04(\mathrm{~m}, 1 \mathrm{H}), 2.77$ (app ddt, $J=13.6,9.6,2.4 \mathrm{~Hz}, 1 \mathrm{H}), 2.10$ (s, 1H), 2.07-1.97 (m, 1H), 1.89 (app ddt, $J=8.6,5.2,1.6 \mathrm{~Hz}, 1 \mathrm{H}), 1.76-1.43$ (comp, 4H).

${ }^{13}$ C-NMR $\left(125 \mathrm{MHz}, \mathrm{CDCl}_{3}\right): \delta=160.6,154.6,128.4,123.7,122.5,120.7,111.1,101.3,55.0,46.9,31.2,26.1$, 24.4 .

Accurate Mass (ESI): Calculated for $\mathrm{C}_{13} \mathrm{H}_{16} \mathrm{NO}[\mathrm{M}+\mathrm{H}]^{+}:$202.1226, Found: 202.1162, Spectral Accuracy: 98.0\%. 
2-(Azepan-2-yl)-1-methyl-1H-indole

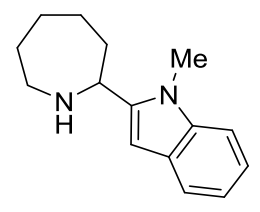

(士)-6h

Following the general procedure E, compound $( \pm)-6 \mathbf{h}$ was obtained from azepane $(113 \mu \mathrm{L}, 1 \mathrm{mmol})$, and trifluoroacetophenone $(191.5 \mathrm{mg}, 1.1 \mathrm{mmol})$. Compound ( \pm )- $\mathbf{6 h}$ was obtained as a colorless oil in $42 \%$ (95.7 $\mathrm{mg})$.

\section{Characterization data:}

$\mathbf{R}_{\mathbf{f}}=0.55$ in EtOAc/MeOH $/ i-\mathrm{PrNH}_{2}$ 90:9:1 v/v/v)

${ }^{1}$ H-NMR $\left(500 \mathrm{MHz}, \mathrm{CDCl}_{3}\right): \delta=7.64-7.52(\mathrm{~m}, 1 \mathrm{H}), 7.31(\mathrm{dd}, J=8.2,1.4 \mathrm{~Hz}, 1 \mathrm{H}), 7.24-7.17(\mathrm{~m}, 1 \mathrm{H}), 7.13-7.07$ (m, 1H), $6.44(\mathrm{~d}, J=1.2 \mathrm{~Hz}, 1 \mathrm{H}), 4.04(\mathrm{dd}, J=9.8,4.1 \mathrm{~Hz}, 1 \mathrm{H}), 3.78(\mathrm{~s}, 3 \mathrm{H}), 3.22-3.08(\mathrm{~m}, 1 \mathrm{H}), 2.95-2.81(\mathrm{~m}$, 1H), 2.18 (app ddt, $J=14.2,7.3,3.5 \mathrm{~Hz}, 1 \mathrm{H}$ ), 2.07-1.88 (comp, 2H), 1.85-1.52 (comp, 6H).

${ }^{13}$ C-NMR (125 MHz, $\left.\mathrm{CDCl}_{3}\right): \delta=144.6,137.7,127.6,121.1,120.3,119.3,108.9,97.9,55.7,47.4,35.9,31.7,30.0$, 27.4, 26.2.

Accurate Mass (ESI): Calculated for $\mathrm{C}_{15} \mathrm{H}_{21} \mathrm{~N}_{2}[\mathrm{M}+\mathrm{H}]^{+}:$229.1699, Found: 229.1794, Spectral Accuracy: 98.0\%. 


\section{1-Methyl-2-(trans-(4-methylpiperidin-2-yl))-1H-indole}

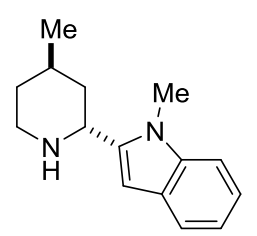

$( \pm)-6 \mathbf{i}$

Following the general procedure E, compound ( \pm )-6i was obtained from 4-methylpiperidine (118 $\mu \mathrm{L}, 1 \mathrm{mmol})$, and trifluoroacetophenone (191.5 mg, $1.1 \mathrm{mmol})$. Compound $( \pm)$-6i was obtained as a colorless oil in 54\% (123.2 mg).

\section{Characterization data:}

$\mathbf{R}_{\mathbf{f}}=0.35$ in EtOAc/MeOH/i-PrNH $\left.290: 9: 1 \mathrm{v} / \mathrm{v} / \mathrm{v}\right)$

${ }^{1}$ H-NMR $\left(500 \mathrm{MHz}, \mathrm{CDCl}_{3}\right): \delta=7.59(\mathrm{~d}, J=7.8 \mathrm{~Hz}, 1 \mathrm{H}), 7.31(\mathrm{~d}, J=8.2 \mathrm{~Hz}, 1 \mathrm{H}), 7.20(\mathrm{ddd}, J=8.2,7.1,1.4 \mathrm{~Hz}$, 1H), 7.13-7.04 (m, 1H), $6.46(\mathrm{~s}, 1 \mathrm{H}), 4.20(\mathrm{dd}, J=8.9,3.6 \mathrm{~Hz}, 1 \mathrm{H}), 3.79(\mathrm{~s}, 3 \mathrm{H}), 3.01$ (ddd, $J=13.0,9.8,3.3 \mathrm{~Hz}$, 1H), 2.93 (app dt, $J=12.8,4.7 \mathrm{~Hz}, 1 \mathrm{H}$ ), 2.32-2.19 (m, 1H), 2.04 (ddd, $J=13.4,8.9,4.5 \mathrm{~Hz}, 1 \mathrm{H}$ ), 1.95-1.65 (comp, $3 \mathrm{H}), 1.42-1.33(\mathrm{~m}, 1 \mathrm{H}), 1.16(\mathrm{~d}, J=7.1 \mathrm{~Hz}, 3 \mathrm{H})$.

${ }^{13}$ C-NMR $\left(125 \mathrm{MHz}, \mathrm{CDCl}_{3}\right): \delta=143.0,137.7,127.5,121.2,120.3,119.4,108.9,98.9,48.4,41.7,38.2,33.1,30.1$, 26.6, 19.3.

Accurate Mass (ESI): Calculated for $\mathrm{C}_{15} \mathrm{H}_{21} \mathrm{~N}_{2}[\mathrm{M}+\mathrm{H}]^{+}: 229.1699$, Found: 229.1744, Spectral Accuracy: 98.7\%. 


\section{2-Benzylpyrrolidine}

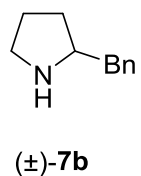

Following the general procedure A, compound $( \pm)-7 \mathbf{b}$ was obtained from pyrrolidine $(82 \mu \mathrm{L}, 1 \mathrm{mmol})$, trifluoroacetophenone $(208.8 \mathrm{mg}, 1.2 \mathrm{mmol})$, benzylmagnesium chloride $\left(1.5 \mathrm{~mL}, 1 \mathrm{M}\right.$ in Et $\left.{ }_{2} \mathrm{O}\right)$, and TMSOTf $(217$ $\mu \mathrm{L}, 1.2 \mathrm{mmol})$. Compound $( \pm)-7 \mathbf{b}$ was obtained as a colorless oil in $56 \%$ yield $(90.2 \mathrm{mg})$.

\section{Characterization data:}

$\mathbf{R}_{\mathbf{f}}=0.11$ in $\mathrm{EtOAc} / \mathrm{MeOH} / i-\mathrm{PrNH}_{2}$ 90:9:1 v/v/v)

${ }^{1} \mathbf{H}-\mathbf{N M R}\left(500 \mathrm{MHz}, \mathrm{CDCl}_{3}\right.$ ): $\delta=7.36-7.27$ (comp, 2H), 7.26-7.19 (comp, 3H), 3.33-3.19 (m, $\left.1 \mathrm{H}\right), 3.06$ (ddd, $J=$ 10.2, 7.7, 5.0 Hz, 1H), 2.85 (ddd, $J=10.3,8.2,6.6 \mathrm{~Hz}, 1 \mathrm{H}), 2.81-2.74$ (comp, 2H), 2.06 (s, 1H), 1.91-1.78 (comp, 2H), 1.74 (dddd, $J=11.0,8.6,6.2,3.4 \mathrm{~Hz}, 1 \mathrm{H}), 1.48-1.35(\mathrm{~m}, 1 \mathrm{H})$.

${ }^{13}$ C-NMR $\left(125 \mathrm{MHz}, \mathrm{CDCl}_{3}\right): \delta=140.3,129.1,128.5,126.2,60.6,46.3,42.5,31.3,24.9$.

Accurate Mass (ESI): Calculated for $\mathrm{C}_{11} \mathrm{H}_{16} \mathrm{~N}[\mathrm{M}+\mathrm{H}]^{+}:$162.1277, Found: 162.1184 , Spectral Accuracy: $98.6 \%$. 


\section{2-Benzylpiperidine}

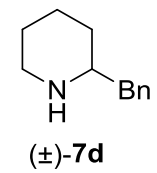

Following the general procedure A, compound $( \pm)-7 \mathbf{d}$ was obtained from piperidine $(99 \mu \mathrm{L}, 1 \mathrm{mmol})$, trifluoroacetophenone (208.8 mg, $1.2 \mathrm{mmol}$ ), benzylmagnesium chloride (1.5 mL, $1 \mathrm{M}$ in $\left.\mathrm{Et}_{2} \mathrm{O}\right)$, and TMSOTf (217 $\mu \mathrm{L}, 1.2 \mathrm{mmol})$. Compound $( \pm)-7 \mathbf{d}$ was obtained as a colorless oil in $70 \%$ yield $(122.5 \mathrm{mg})$.

\section{Characterization data:}

$\mathbf{R}_{\mathbf{f}}=0.13$ in $\mathrm{EtOAc} / \mathrm{MeOH} / i-\mathrm{PrNH}_{2}$ 90:9:1 v/v/v)

${ }^{1} \mathbf{H}-\mathbf{N M R}\left(500 \mathrm{MHz}, \mathrm{CDCl}_{3}\right.$ ): $\delta=7.33-7.23(\mathrm{~m}, 1 \mathrm{H}), 7.23-7.00$ (comp, 4H), 3.06-2.79 (m, 1H), 2.78-2.26 (comp, 4H), 1.87-1.48 (comp,4H), 1.45-1.39 (m, 1H), 1.35-0.97 (comp, 2H).

${ }^{13}$ C-NMR (125 MHz, $\left.\mathrm{CDCl}_{3}\right): \delta=139.2,129.2,128.4,126.2,58.3,47.2,43.9,32.9,26.2,24.9$.

Accurate Mass (ESI): Calculated for $\mathrm{C}_{12} \mathrm{H}_{18} \mathrm{~N}[\mathrm{M}+\mathrm{H}]^{+}$: 176.1434, Found: 176.1337, Spectral Accuracy: 98.6\%. 


\section{1-Methyl-3-phenylpiperazine}

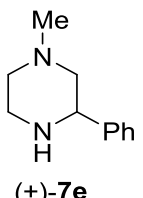

$( \pm)-7 e$

Following the general procedure A, compound $( \pm)-7 \mathbf{e}$ was obtained from1-methylpiperazine $(111 \mu \mathrm{L}, 1 \mathrm{mmol})$,

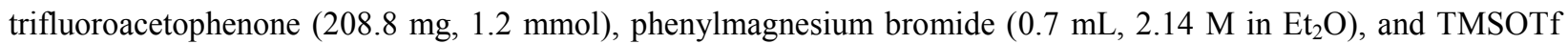
$(217 \mu \mathrm{L}, 1.2 \mathrm{mmol})$. Compound ( \pm )-7e was obtained as a white solid in $46 \%$ yield $(81 \mathrm{mg})$.

\section{Characterization data:}

$\mathbf{R}_{\mathbf{f}}=0.14$ in EtOAc/MeOH/i-PrNH $\left.290: 9: 1 \mathrm{v} / \mathrm{v} / \mathrm{v}\right)$

${ }^{1}$ H-NMR (500 MHz, $\mathrm{CDCl}_{3}$ ): $\delta=7.37-7.32$ (comp, 2H), 7.30-7.26 (comp, 2H), 7.24-7.20 (m, 1H), 3.88-3.78 (m, 1H), 3.13-2.96 (comp, 2H), 2.83 (ddd, $J=11.1,3.0,1.4 \mathrm{~Hz}, 1 \mathrm{H}), 2.80-2.75$ (m, 1H), 2.27 (s, 3H), 2.10 (app tdd, $J=$ 11.7, 11.0, 4.0, 1.2 Hz, 1H), 1.99-1.93 (m, 1H), 1.83 (s, 1H).

${ }^{13}$ C-NMR $\left(125 \mathrm{MHz}, \mathrm{CDCl}_{3}\right): \delta=142.6,128.5,127.6,127.1,63.4,60.5,55.4,46.5,46.4$.

Accurate Mass (ESI): Calculated for $\mathrm{C}_{11} \mathrm{H}_{17} \mathrm{~N}_{2}[\mathrm{M}+\mathrm{H}]^{+}: 177.1386$, Found: 177.1252 , Spectral Accuracy: $98.3 \%$ 


\section{2-Benzylazepane}

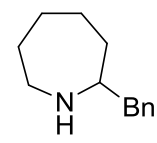

$$
( \pm)-7 f
$$

Following the general procedure A, compound $( \pm)-7 \mathbf{f}$ was obtained from azepane $(113 \mu \mathrm{L}, 1 \mathrm{mmol})$, trifluoroacetophenone (208.8 mg, $1.2 \mathrm{mmol})$, benzylmagnesium chloride (1.5 mL, $1 \mathrm{M}$ in $\left.\mathrm{Et}_{2} \mathrm{O}\right)$, and TMSOTf (217 $\mu \mathrm{L}, 1.2 \mathrm{mmol})$. Compound $( \pm)-7 \mathbf{f}$ was obtained as a colorless oil in $55 \%$ yield $(103.9 \mathrm{mg})$.

\section{Characterization data:}

$\mathbf{R}_{\mathbf{f}}=0.28$ in $\mathrm{EtOAc} / \mathrm{MeOH} / i-\mathrm{PrNH}_{2}$ 90:9:1 $\left.\mathrm{v} / \mathrm{v} / \mathrm{v}\right)$

${ }^{1}$ H-NMR (500 MHz, $\mathrm{CDCl}_{3}$ ): $\delta=7.36-7.28$ (comp, 2H), 7.26-7.18 (comp, 3H), 3.01-2.94 (m, 1H), 2.91 (app tdd, $J$ $=9.1,5.5,3.8 \mathrm{~Hz}, 1 \mathrm{H}), 2.74$ (dd, $J=13.5,5.5 \mathrm{~Hz}, 1 \mathrm{H}), 2.62$ (comp, 2H), 1.95-1.79 (comp, 2H), 1.77-1.58 (comp, 5H), 1.58-1.47 (m, 1H), 1.41 (app dtd, $J=13.1,9.5,3.3 \mathrm{~Hz}, 1 \mathrm{H})$.

${ }^{13}$ C-NMR $\left(125 \mathrm{MHz}, \mathrm{CDCl}_{3}\right): \delta=139.8,129.2,128.5,126.2,61.0,47.7,44.1,36.8,30.6,27.1,25.4$.

Accurate Mass (ESI): Calculated for $\mathrm{C}_{13} \mathrm{H}_{20} \mathrm{~N}[\mathrm{M}+\mathrm{H}]^{+}:$190.1590, Found: 190.1432, Spectral Accuracy: 98.4\%. 


\section{2-Methylazacyclotridecane}

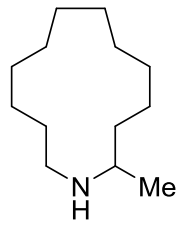

$( \pm)-7 g$

Following the general procedure B, compound $( \pm)-7 \mathrm{~g}$ was obtained from azacyclotridecane $(91.6 \mathrm{mg}, 0.5 \mathrm{mmol})$,

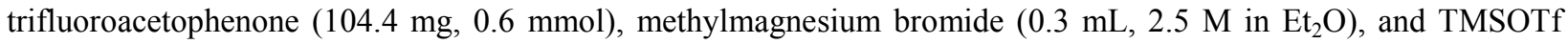
$(108.5 \mu \mathrm{L}, 0.6 \mathrm{mmol})$. Compound $( \pm)-7 \mathrm{~g}$ was obtained as a colorless oil in $68 \%$ yield $(67 \mathrm{mg})$.

\section{Characterization data:}

$\mathbf{R}_{\mathbf{f}}=0.24$ in EtOAc/Hexanes $\left.60: 40 \mathrm{v} / \mathrm{v}\right)$

${ }^{1}$ H-NMR $\left(500 \mathrm{MHz}, \mathrm{CDCl}_{3}\right): \delta=2.78(\mathrm{ddd}, J=12.1,5.9,4.0 \mathrm{~Hz}, 1 \mathrm{H}), 2.69-2.59(\mathrm{~m}, 1 \mathrm{H}), 2.52(\mathrm{ddd}, J=12.2,8.4$, $3.8 \mathrm{~Hz}, 1 \mathrm{H}), 1.72-1.23$ (comp, 21H), $1.02(\mathrm{~d}, J=6.3 \mathrm{~Hz}, 3 \mathrm{H})$.

${ }^{13}$ C-NMR $\left(125 \mathrm{MHz}, \mathrm{CDCl}_{3}\right): \delta=52.1,45.6,35.8,28.3,26.6(2)$, 26.5, 25.8, 25.3, 25.1, 24.4, 23.6, 21.6.

Accurate Mass (ESI): Calculated for $\mathrm{C}_{13} \mathrm{H}_{28} \mathrm{~N}[\mathrm{M}+\mathrm{H}]^{+}:$198.2216, Found: 198.2137, Spectral Accuracy: $98.7 \%$. 


\section{2-Butylazacyclotridecane}

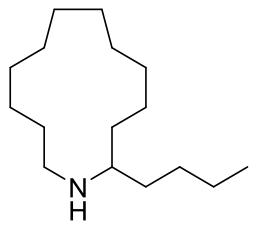

$( \pm)-7 h$

Following the general procedure B, compound $( \pm)-7 \mathbf{h}$ was obtained from azacyclotridecane $(91.6 \mathrm{mg}, 0.5 \mathrm{mmol})$, trifluoroacetophenone $(104.4 \mathrm{mg}, 0.6 \mathrm{mmol})$, butylmagnesium chloride $\left(0.47 \mathrm{~mL}, 1.6 \mathrm{M}^{\text {in }} \mathrm{Et}_{2} \mathrm{O}\right)$, and TMSOTf $(108.5 \mu \mathrm{L}, 0.6 \mathrm{mmol})$. Compound $( \pm)-7 \mathbf{h}$ was obtained as a colorless oil in $87 \%$ yield $(104 \mathrm{mg})$.

\section{Characterization data:}

$\mathbf{R}_{\mathbf{f}}=0.32$ in EtOAc/Hexanes 10:90 v/v)

${ }^{1}$ H-NMR $\left(500 \mathrm{MHz}, \mathrm{CDCl}_{3}\right): \delta=2.74(\mathrm{ddd}, J=11.9,5.6,4.3 \mathrm{~Hz}, 1 \mathrm{H}), 2.53(\mathrm{ddd}, J=11.9,8.8,3.9 \mathrm{~Hz}, 1 \mathrm{H}), 2.41$ (app ddt, $J=9.1,5.9,2.9 \mathrm{~Hz}, 1 \mathrm{H}), 1.60-1.17$ (comp, 27H), 0.89 (t, $J=7.0 \mathrm{~Hz}, 3 \mathrm{H}$ ).

${ }^{13}$ C-NMR (125 MHz, $\left.\mathrm{CDCl}_{3}\right): \delta=57.4,45.7,35.5,33.5,28.8,28.7,26.9,26.6,26.4,25.6,25.2,25.1,24.3,23.5$, $23.2,14.3$.

Accurate Mass (ESI): Calculated for $\mathrm{C}_{16} \mathrm{H}_{34} \mathrm{~N}[\mathrm{M}+\mathrm{H}]^{+}: 240.2686$, Found: 240.2685 , Spectral Accuracy: $98.8 \%$. 


\section{2-Hexylazacyclotridecane}

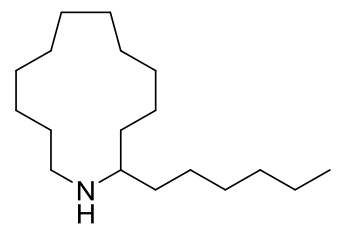

$( \pm)-7 \mathbf{i}$

Following the general procedure B, compound $( \pm)-7 \mathbf{i}$ was obtained from azacyclotridecane $(91.6 \mathrm{mg}, 0.5 \mathrm{mmol})$, trifluoroacetophenone $(104.4 \mathrm{mg}, 0.6 \mathrm{mmol})$, hexylmagnesium bromide $\left(0.5 \mathrm{~mL}, 1.5 \mathrm{M}\right.$ in $\left.\mathrm{Et}_{2} \mathrm{O}\right)$, and TMSOTf $(108.5 \mu \mathrm{L}, 0.6 \mathrm{mmol})$. Compound $( \pm)-7 \mathbf{i}$ was obtained as a colorless oil in $66 \%$ yield $(88.1 \mathrm{mg})$.

\section{Characterization data:}

$\mathbf{R}_{\mathbf{f}}=0.60$ in EtOAc/Hexanes $60: 40 \mathrm{v} / \mathrm{v}$ )

${ }^{1}$ H-NMR (500 MHz, $\left.\mathrm{CDCl}_{3}\right): \delta=2.76(\mathrm{dt}, J=11.9,4.9 \mathrm{~Hz}, 1 \mathrm{H}), 2.62-2.49(\mathrm{~m}, 1 \mathrm{H}), 2.49-2.39(\mathrm{~m}, 1 \mathrm{H}), 1.58-1.41$ (comp, 6H), 1.41-1.23 (comp, 25H), 0.89 (t, $J=6.8 \mathrm{~Hz}, 3 \mathrm{H}$ ).

${ }^{13}$ C-NMR $\left(125 \mathrm{MHz}, \mathrm{CDCl}_{3}\right): \delta=57.4,45.6,35.7,33.4,32.0,29.8,28.7,26.9,26.5,26.4,26.4,25.5,25.2,25.0$, $24.3,23.5,22.8,14.2$.

Accurate Mass (ESI): Calculated for $\mathrm{C}_{18} \mathrm{H}_{38} \mathrm{~N}[\mathrm{M}+\mathrm{H}]^{+}:$268.2999, Found: 268.2948, Spectral Accuracy: 99.0\%. 


\section{2-Isobutylazacyclotridecane}

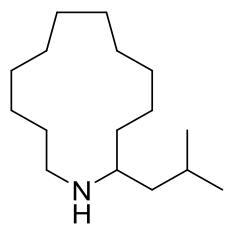

$( \pm)-7 \mathbf{j}$

Following the general procedure B, compound $( \pm)-7 \mathbf{j}$ was obtained from azacyclotridecane $(91.6 \mathrm{mg}, 0.5 \mathrm{mmol})$, trifluoroacetophenone $(104.4 \mathrm{mg}, 0.6 \mathrm{mmol})$, isobutylmagnesium bromide $\left(0.5 \mathrm{~mL}, 1.5 \mathrm{M}^{\left.\text {in } \mathrm{Et}_{2} \mathrm{O}\right) \text {, and TMSOTf }}\right.$ (108.5 $\mu \mathrm{L}, 0.6 \mathrm{mmol})$. Compound $( \pm)-7 \mathbf{j}$ was obtained as a colorless oil in $68 \%$ yield $(81.3 \mathrm{mg})$.

\section{Characterization data:}

$\mathbf{R}_{\mathbf{f}}=0.33$ in EtOAc/Hexanes $60: 40 \mathrm{v} / \mathrm{v}$ )

${ }^{1}$ H-NMR (500 MHz, $\mathrm{CDCl}_{3}$ ): $\delta=2.73$ (app dt, $J=12.2,5.0 \mathrm{~Hz}, 1 \mathrm{H}$ ), 2.61-2.41 (comp, 2H), 1.63 (app dt, $J=13.4$, $6.7 \mathrm{~Hz}, 1 \mathrm{H}), 1.55-1.41$ (comp, 4H), 1.42-1.24 (comp, 16H), 1.23-1.04 (comp, 3H), 0.89 (d, $J=2.9 \mathrm{~Hz}, 3 \mathrm{H}), 0.87$ $(\mathrm{d}, J=3.0 \mathrm{~Hz}, 3 \mathrm{H})$.

${ }^{13}$ C-NMR $\left(125 \mathrm{MHz}, \mathrm{CDCl}_{3}\right): \delta=55.1,45.5,45.4,33.7,28.9,27.0,26.6,26.3,25.6,25.3,25.1,25.2,24.3,23.4$, 23.3, 22.9 .

Accurate Mass (ESI): Calculated for $\mathrm{C}_{16} \mathrm{H}_{34} \mathrm{~N}[\mathrm{M}+\mathrm{H}]^{+}: 240.2686$, Found: 240.2675, Spectral Accuracy: 98.6\%. 


\section{2-Isopropylazacyclotridecane}

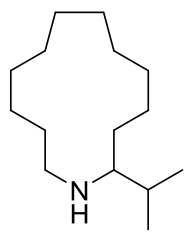

$( \pm)-7 k$

Following the general procedure B, compound $( \pm)-7 \mathbf{k}$ was obtained from azacyclotridecane $(91.6 \mathrm{mg}, 0.5 \mathrm{mmol})$, trifluoroacetophenone $(104.4 \mathrm{mg}, 0.6 \mathrm{mmol})$, isopropylmagnesium chloride $(0.42 \mathrm{~mL}, 1.8 \mathrm{M}$ in THF), and TMSOTf $(108.5 \mu \mathrm{L}, 0.6 \mathrm{mmol})$. Compound $( \pm)-7 \mathbf{k}$ was obtained as a colorless oil in $62 \%$ yield $(69.8 \mathrm{mg})$.

\section{Characterization data:}

$\mathbf{R}_{\mathbf{f}}=0.14 \mathrm{in} \mathrm{EtOAc/Hexanes} \mathrm{60:40} \mathrm{v/v)}$

${ }^{1}$ H-NMR (500 MHz, $\mathrm{CDCl}_{3}$ ): $\delta=2.83-2.67(\mathrm{~m}, 1 \mathrm{H}), 2.59-2.46(\mathrm{~m}, 1 \mathrm{H}), 2.18(\mathrm{ddd}, J=9.8,5.0,2.5 \mathrm{~Hz}, 1 \mathrm{H}), 1.72$ (ddd, $J=8.6,6.8,3.4 \mathrm{~Hz}, 1 \mathrm{H}), 1.57-1.42$ (comp, 5H), 1.44-1.28 (comp, 16H), 0.88 (dd, $J=6.9,1.9 \mathrm{~Hz}, 3 \mathrm{H}), 0.84$ $(\mathrm{dd}, J=6.8,2.0 \mathrm{~Hz}, 3 \mathrm{H})$.

${ }^{13}$ C-NMR $\left(125 \mathrm{MHz}, \mathrm{CDCl}_{3}\right): \delta=63.2,46.7,31.4,30.5,28.9,26.9,26.5,26.4,25.4,24.8,24.6,24.4,24.0,19.5$, 18.1 .

Accurate Mass (ESI): Calculated for $\mathrm{C}_{15} \mathrm{H}_{32} \mathrm{~N}[\mathrm{M}+\mathrm{H}]^{+}:$226.2529, Found: 226.2594, Spectral Accuracy: $98.6 \%$. 


\section{2-Allylazacyclotridecane}

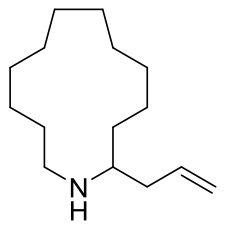

$( \pm)-71$

Following the general procedure B, compound $( \pm)-71$ was obtained from azacyclotridecane $(91.6 \mathrm{mg}, 0.5 \mathrm{mmol})$, trifluoroacetophenone (104.4 mg, $0.6 \mathrm{mmol})$, allylmagnesium chloride ( $1 \mathrm{~mL}, 0.75 \mathrm{M}$ in MeTHF), and TMSOTf $(108.5 \mu \mathrm{L}, 0.6 \mathrm{mmol})$. Compound $( \pm)-71$ was obtained as a colorless oil in $60 \%$ yield $(66.9 \mathrm{mg})$.

\section{Characterization data:}

$\mathbf{R}_{\mathbf{f}}=0.33$ in EtOAc/Hexanes $\left.60: 40 \mathrm{v} / \mathrm{v}\right)$

${ }^{1}$ H-NMR $\left(500 \mathrm{MHz}, \mathrm{CDCl}_{3}\right): \delta=5.78$ (app ddt, $\left.J=17.3,10.3,7.2 \mathrm{~Hz}, 1 \mathrm{H}\right), 5.09-5.03$ (m, 1H), 5.02 (app q, $J=1.6$, $1.1 \mathrm{~Hz}, 1 \mathrm{H}$ ), 2.76 (app dt, $J=12.1,5.2 \mathrm{~Hz}, 1 \mathrm{H}$ ), 2.60-2.45 (comp, 2H), 2.18 (app dddt, $J=13.4,7.3,6.0,1.3 \mathrm{~Hz}$, 1H), 2.13-2.01 (m, 1H), 1.56-1.44 (comp, 4H), 1.43-1.22 (comp, 17H).

${ }^{13} \mathbf{C - N M R}\left(125 \mathrm{MHz}, \mathrm{CDCl}_{3}\right): \delta=136.5,116.8,56.8,45.7,40.1,33.3,28.7,26.7,26.5,26.4,25.6,25.2,25.0,24.4$, 23.5.

Accurate Mass (ESI): Calculated for $\mathrm{C}_{15} \mathrm{H}_{30} \mathrm{~N}[\mathrm{M}+\mathrm{H}]^{+}: 224.2373$, Found: 224.2379, Spectral Accuracy: $98.9 \%$. 


\section{2-Vinylazacyclotridecane}

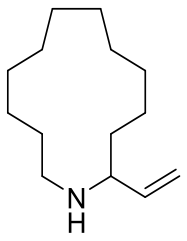

$( \pm)-7 m$

Following the general procedure B, compound $( \pm)-7 \mathbf{m}$ was obtained from azacyclotridecane $(91.6 \mathrm{mg}, 0.5 \mathrm{mmol})$, trifluoroacetophenone (104.4 mg, $0.6 \mathrm{mmol}$ ), vinylmagnesium bromide ( $1 \mathrm{~mL}, 0.75 \mathrm{M}$ in THF), and TMSOTf $(108.5 \mu \mathrm{L}, 0.6 \mathrm{mmol})$. Compound $( \pm)-7 \mathrm{~m}$ was obtained as a colorless oil in $67 \%$ yield $(70 \mathrm{mg})$.

\section{Characterization data:}

$\mathbf{R}_{\mathbf{f}}=0.32$ in EtOAc/Hexanes $60: 40 \mathrm{v} / \mathrm{v}$ )

${ }^{1}$ H-NMR (500 MHz, $\mathrm{CDCl}_{3}$ ): $\delta=5.66(\mathrm{ddd}, J=17.4,10.2,7.5 \mathrm{~Hz}, 1 \mathrm{H}), 5.06$ (app dt, $\left.J=17.2,1.4 \mathrm{~Hz}, 1 \mathrm{H}\right), 5.00$ (dd, $J=10.2,1.9 \mathrm{~Hz}, 1 \mathrm{H}), 3.09-2.96(\mathrm{~m}, 1 \mathrm{H}), 2.73$ (ddd, $J=12.4,6.3,3.9 \mathrm{~Hz}, 1 \mathrm{H}), 2.49$ (ddd, $J=12.3,8.6,3.4 \mathrm{~Hz}$, 1H), 1.63-1.45 (comp, 3H), 1.45-1.22 (comp, 18H).

${ }^{13}$ C-NMR $\left(125 \mathrm{MHz}, \mathrm{CDCl}_{3}\right): \delta=142.42,113.88,60.1,45.6,34.5,28.1,26.8,26.6,26.4,25.9,25.3,25.2,24.5$, 23.8 .

Accurate Mass (ESI): Calculated for $\mathrm{C}_{14} \mathrm{H}_{28} \mathrm{~N}[\mathrm{M}+\mathrm{H}]^{+}: 210.2216$, Found: 210.2149, Spectral Accuracy: 98.9\%. 


\section{2-Ethynylazacyclotridecane}

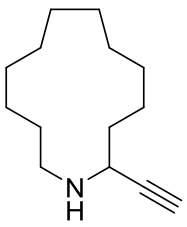

$( \pm)-7 n$

Following the general procedure B, compound $( \pm)-\mathbf{7 n}$ was obtained from azacyclotridecane $(91.6 \mathrm{mg}, 0.5 \mathrm{mmol})$, trifluoroacetophenone (104.4 mg, $0.6 \mathrm{mmol})$, ethynylmagnesium bromide (1.5 mL, $0.5 \mathrm{M}$ in THF), and TMSOTf $(108.5 \mu \mathrm{L}, 0.6 \mathrm{mmol})$. Compound $( \pm)-7 \mathbf{n}$ was obtained as a colorless oil in $70 \%$ yield $(72.5 \mathrm{mg})$.

\section{Characterization data:}

$\mathbf{R}_{\mathbf{f}}=0.9$ in EtOAc/Hexanes 60:40 v/v)

${ }^{1}$ H-NMR $\left(500 \mathrm{MHz} \mathrm{CDCl}_{3}\right): \delta=3.34(\mathrm{ddd}, J=9.7,3.5,2.1 \mathrm{~Hz}, 1 \mathrm{H}), 2.85(\mathrm{ddd}, J=12.2,7.0,3.4 \mathrm{~Hz}, 1 \mathrm{H}), 2.63$ (ddd, $J=11.7,7.8,3.0 \mathrm{~Hz}, 1 \mathrm{H}), 2.21(\mathrm{~d}, J=2.1 \mathrm{~Hz}, 1 \mathrm{H}), 1.76-1.67(\mathrm{~m}, 1 \mathrm{H}), 1.66-1.57$ (comp, 2H), $1.56-1.47$ (comp, 2H), 1.46-1.22 (comp, 16H).

${ }^{13}$ C-NMR $\left(125 \mathrm{MHz}, \mathrm{CDCl}_{3}\right): \delta=87.06,70.0,48.4,45.7,35.0,28.1,26.8,26.7,26.1,25.5,25.4,24.8,23.8$.

Accurate Mass (ESI): Calculated for $\mathrm{C}_{14} \mathrm{H}_{26} \mathrm{~N}[\mathrm{M}+\mathrm{H}]^{+}:$208.2060, Found: 208.1933, Spectral Accuracy: $98.4 \%$. 
trans-4-Benzyl-2-(but-3-en-1-yl)piperidine

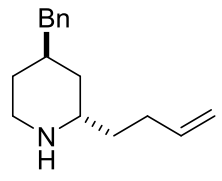

$( \pm)-7 p$

Following the general procedure B, compound $( \pm)-7 \mathbf{p}$ was obtained from 4-benzyl piperidine $(87.5 \mathrm{mg}, 0.5 \mathrm{mmol})$, trifluoroacetophenone (104.4 mg, $0.6 \mathrm{mmol})$, but-3-en-1-ylmagnesium bromide ( $0.86 \mathrm{~mL}, 0.87 \mathrm{M}$ in THF), and TMSOTf $(108.5 \mu \mathrm{L}, 0.6 \mathrm{mmol})$. Compound $( \pm)-7 \mathbf{p}$ was obtained as a colorless oil in $56 \%$ yield $(64.2 \mathrm{mg})$.

\section{Characterization data:}

$\mathbf{R}_{\mathbf{f}}=0.13$ in EtOAc $/ \mathrm{MeOH} / i-\mathrm{PrNH}_{2}$ 90:9:1 v/v/v)

${ }^{1}$ H-NMR (500 MHz, CDCl $): \delta=7.40-7.33(\mathrm{comp}, 2 \mathrm{H}), 7.30-7.26(\mathrm{~m}, 1 \mathrm{H}), 7.25-7.22(\mathrm{comp}, 2 \mathrm{H}), 5.91$ (ddt, $J=$ $16.9,10.2,6.6 \mathrm{~Hz}, 1 \mathrm{H}), 5.11(\mathrm{dq}, J=17.1,1.7 \mathrm{~Hz}, 1 \mathrm{H}), 5.05$ (ddt, $J=10.2,2.2,1.3 \mathrm{~Hz}, 1 \mathrm{H}), 3.01-2.95$ (comp, 2H), 2.92 (ddd, $J=12.4,6.0,4.2 \mathrm{~Hz}, 1 \mathrm{H}$ ), 2.77-2.72 (comp, 2H), 2.25-2.03 (comp, 3H), 1.80-1.68 (comp, 2H), 1.671.55 (comp, 2H), 1.54-1.40 (comp, 3H).

${ }^{13} \mathbf{C}-\mathbf{N M R}\left(125 \mathrm{MHz}, \mathrm{CDCl}_{3}\right): \delta=141.2,138.7,129.1,128.3,125.8,114.6,50.9,41.2,40.2,36.5,34.3,33.6,31.2$, 30.6 .

Accurate Mass (ESI): Calculated for $\mathrm{C}_{16} \mathrm{H}_{24} \mathrm{~N}[\mathrm{M}+\mathrm{H}]^{+}:$230.1903, Found: 230.1906, Spectral Accuracy: 99\%. 


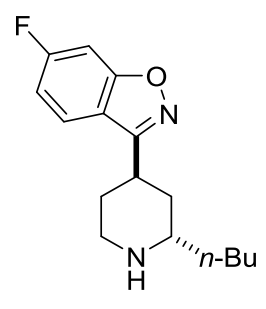

$( \pm)-7 q$

Following the general procedure B, compound ( \pm )-7q was obtained from 6-fluoro-3-(piperidin-4yl)benzo[d]isoxazole (110 mg, $0.5 \mathrm{mmol})$, trifluoroacetophenone (104.4 mg, $0.6 \mathrm{mmol})$, butylmagnesium chloride

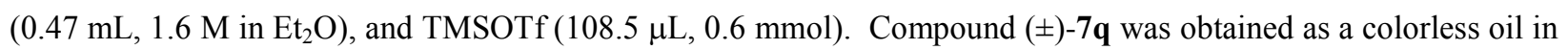
$52 \%$ yield $(71.7 \mathrm{mg})$.

\section{Characterization data:}

$\mathbf{R}_{\mathbf{f}}=0.26$ in EtOAc/MeOH/i-PrNH 2 90:9:1 v/v/v)

${ }^{1}$ H-NMR $\left(500 \mathrm{MHz}, \mathrm{CDCl}_{3}\right): \delta=7.71(\mathrm{dd}, J=8.7,5.1 \mathrm{~Hz}, 1 \mathrm{H}), 7.34(\mathrm{dd}, J=8.5,2.1 \mathrm{~Hz}, 1 \mathrm{H}), 7.15(\operatorname{app} \mathrm{td}, J=8.8$, $2.1 \mathrm{~Hz}, 1 \mathrm{H}), 3.76-3.53(\mathrm{~m}, 1 \mathrm{H}), 3.21-3.04(\mathrm{comp}, 3 \mathrm{H}), 2.40-2.23(\mathrm{~m}, 1 \mathrm{H}), 2.25-2.03$ (comp, 3H), 1.85 (ddd, $J=$ $13.7,9.0,5.0 \mathrm{~Hz}, 1 \mathrm{H}), 1.63-1.47$ (comp, 2H), 1.48-1.28 (comp, 4H), 0.98 (t, $J=6.9 \mathrm{~Hz}, 3 \mathrm{H})$.

${ }^{13} \mathrm{C}-\mathrm{NMR}\left(125 \mathrm{MHz}, \mathrm{CDCl}_{3}\right): \delta=164.2\left(\mathrm{~d}, J_{C-F}=250.5 \mathrm{~Hz}\right), 163.7\left(\mathrm{~d}, J_{C-F}=13.5 \mathrm{~Hz}\right), 160.9,122.5\left(\mathrm{~d}, J_{C-F}=11.0\right.$ $\mathrm{Hz}), 117.9\left(\mathrm{~d}, J_{C-F}=1.3 \mathrm{~Hz}\right), 112.4\left(\mathrm{~d}, J_{C-F}=25.3 \mathrm{~Hz}\right), 97.5\left(\mathrm{~d}, J_{C-F}=26.8 \mathrm{~Hz}\right), 51.9,41.9,35.1,34.9,30.7,29.6$, 28.3, 22.9, 14.2.

Accurate Mass (ESI): Calculated for $\mathrm{C}_{16} \mathrm{H}_{22} \mathrm{~N}_{2} \mathrm{FO}[\mathrm{M}+\mathrm{H}]^{+}$: 277.1711, Found: 277.1600, Spectral Accuracy: $98.6 \%$. 


\section{2-(2,6-Dichlorophenyl)pyrrolidine}

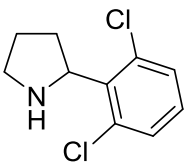

$( \pm)-8 \mathbf{a}$

Following general procedure A, compound $( \pm)-8 \mathbf{a}$ was obtained from pyrrolidine $(82 \mu \mathrm{L}, 1 \mathrm{mmol})$, trifluoroacetophenone $(191.4 \mathrm{mg}, 1.2 \mathrm{mmol})$, the organometallic nucleophile $(1.5 \mathrm{mmol})$, and $\mathrm{BF}_{3} \cdot \mathrm{OEt}_{2}(148 \mu \mathrm{L}, 1.2$ $\mathrm{mmol})$. Compound $( \pm)-8 \mathbf{a}$ was obtained as a yellow oil in $38 \%$ yield $(81.7 \mathrm{mg})$.

The organometallic nucleophile was prepared as follows: A flame dried vial was charged with $i$-PrMgCl $\cdot \mathrm{LiCl}$ solution in THF $(1.33 \mathrm{~mL}, 1.13 \mathrm{M})$ followed by the addition of 2-bromo-1,3-dichlorobenzene $(338.8 \mathrm{mg}, 1.5 \mathrm{mmol}$, 1.5 equiv) in $0.7 \mathrm{~mL}$ of anhydrous THF. The $\mathrm{Br} / \mathrm{Mg}$ exchange was completed in 1 hour at room temperature.

\section{Characterization data:}

$\mathbf{R}_{\mathbf{f}}=0.13$ in $\mathrm{EtOAc} / \mathrm{MeOH} / i-\mathrm{PrNH}_{2}$ 90:9:1 v/v/v)

${ }^{1}$ H-NMR (500 MHz, $\left.\mathrm{CDCl}_{3}\right): \delta=7.39(\mathrm{dd}, J=7.9,1.8 \mathrm{~Hz}, 2 \mathrm{H}), 7.25-7.14(\mathrm{~m}, 1 \mathrm{H}), 5.03(\operatorname{app~t}, J=8.3 \mathrm{~Hz}, 1 \mathrm{H})$, 3.56-3.33 (m, 1H), 3.07 (app dt, $J=12.0,7.3 \mathrm{~Hz}, 1 \mathrm{H}), 2.72-2.50$ (m, 1H), 2.33-2.07 (comp, 3H), $2.06-1.92$ (m, $1 \mathrm{H})$.

${ }^{13}$ C-NMR $\left(125 \mathrm{MHz}, \mathrm{CDCl}_{3}\right): \delta=137.6,135.3,129.5,128.4,59.4,48.3,30.1,27.5$.

Accurate Mass (ESI): Calculated for $\mathrm{C}_{10} \mathrm{H}_{12} \mathrm{NCl}_{2}[\mathrm{M}+\mathrm{H}]^{+}:$216.0341, Found: 216.0225, 217.9996, Spectral Accuracy: $98.7 \%$. 


\section{2-(2,6-Dichlorophenyl)piperidine}

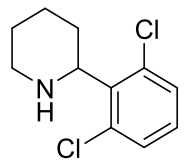

(土)-8b

Following general procedure A, compound $( \pm)-8 \mathbf{b}$ was obtained from piperidine $(99 \mu \mathrm{L}, 1 \mathrm{mmol})$, trifluoroacetophenone (191.4 mg, $1.2 \mathrm{mmol})$, the organometallic nucleophile $(1.5 \mathrm{mmol})$, and $\mathrm{BF}_{3} \cdot \mathrm{OEt}_{2}(148 \mu \mathrm{L}, 1.2$ $\mathrm{mmol})$. Compound $( \pm)-\mathbf{8 b}$ was obtained as a yellow oil in $55 \%$ yield $(125.9 \mathrm{mg})$.

The organometallic nucleophile was prepared as follows: A flame dried vial was charged with $i$-PrMgCl $\cdot \mathrm{LiCl}$ solution in THF $(1.33 \mathrm{~mL}, 1.13 \mathrm{M})$ followed by the addition of 2-bromo-1,3-dichlorobenzene (338.8 mg, $1.5 \mathrm{mmol}$, 1.5 equiv) in $0.7 \mathrm{~mL}$ of anhydrous THF. The $\mathrm{Br} / \mathrm{Mg}$ exchange was completed in 1 hour at room temperature.

\section{Characterization data:}

$\mathbf{R}_{\mathbf{f}}=0.57$ in EtOAc/MeOH$/ i-\mathrm{PrNH}_{2}$ 90:9:1 v/v/v)

${ }^{1}$ H-NMR $\left(500 \mathrm{MHz}, \mathrm{CDCl}_{3}\right): \delta=7.22(\mathrm{~d}, J=8.1 \mathrm{~Hz}, 2 \mathrm{H}), 7.02(\mathrm{t}, J=8.0 \mathrm{~Hz}, 1 \mathrm{H}), 4.49(\mathrm{dd}, J=12.0,2.7 \mathrm{~Hz}, 1 \mathrm{H})$, 3.29-3.13 (m, 1H), $2.72 \mathrm{app}(\mathrm{td}, J=12.9,2.6 \mathrm{~Hz}, 1 \mathrm{H}), 2.35(\mathrm{~s}, 1 \mathrm{H}), 2.11$ (app qd, $J=13.1,12.5,3.5 \mathrm{~Hz}, 1 \mathrm{H}), 1.92$ (app dqd, $J=10.2,3.9,1.8 \mathrm{~Hz}, 1 \mathrm{H}), 1.71-1.50(\mathrm{comp}, 3 \mathrm{H}), 1.41$ (ddd, $J=16.6,8.9,4.0 \mathrm{~Hz}, 1 \mathrm{H})$.

${ }^{13}$ C-NMR $\left(125 \mathrm{MHz}, \mathrm{CDCl}_{3}\right): \delta=138.5,134.8,129.5,128.3,59.4,47.9,28.9,27.1,25.6$.

Accurate Mass (ESI): Calculated for $\mathrm{C}_{11} \mathrm{H}_{14} \mathrm{NCl}_{2}[\mathrm{M}+\mathrm{H}]^{+}$: 230.0498, Found: 230.0575, 232.0344, Spectral Accuracy: 98.4\%. 
2-(Piperidin-2-yl)benzonitrile

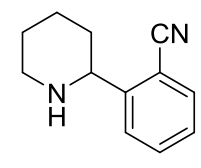

$( \pm)-8 c$

Following general procedure A, compound $( \pm)-8 \mathrm{c}$ was obtained from piperidine (99 $\mu \mathrm{L}, 1 \mathrm{mmol}$ ), trifluoroacetophenone $(191.4 \mathrm{mg}, 1.2 \mathrm{mmol})$, the organometallic nucleophile $(1.5 \mathrm{mmol})$, and $\mathrm{BF}_{3} \cdot \mathrm{OEt}_{2}(148 \mu \mathrm{L}, 1.2$ $\mathrm{mmol})$. Compound $( \pm)-8 \mathbf{c}$ was obtained as a colorless oil in $43 \%$ yield $(80 \mathrm{mg})$.

The organometallic nucleophile was prepared as follows: A flame dried vial was charged with $i$-PrMgCl$\cdot \mathrm{LiCl}$ solution in THF (1.33 mL, $1.13 \mathrm{M}$ ) followed by the addition of 2-bromobenzonitrile (273 $\mathrm{mg}, 1.5 \mathrm{mmol}, 1.5$ equiv) in $0.7 \mathrm{~mL}$ of anhydrous THF. The $\mathrm{Br} / \mathrm{Mg}$ exchange was completed in 1 hour.

\section{Characterization data:}

$\mathbf{R}_{\mathbf{f}}=0.11$ in EtOAc/MeOH/i-PrNH $\left.290: 9: 1 \mathrm{v} / \mathrm{v} / \mathrm{v}\right)$

${ }^{1}$ H-NMR $\left(500 \mathrm{MHz}, \mathrm{CDCl}_{3}\right): \delta=7.75(\mathrm{~d}, J=7.2 \mathrm{~Hz}, 1 \mathrm{H}), 7.50-7.40(\mathrm{comp}, 2 \mathrm{H}), 7.36(\mathrm{~d}, J=7.6 \mathrm{~Hz}, 1 \mathrm{H}), 4.82(\mathrm{~s}$, 1H), 4.50-4.34 (m, 1H), 4.35-4.21 (m, 1H), 3.03 (app td, $J=12.9,3.3 \mathrm{~Hz}, 1 \mathrm{H}), 2.27$ (d, $J=12.4 \mathrm{~Hz}, 1 \mathrm{H}), 1.98$ (d, $J$ $=13.5 \mathrm{~Hz}, 1 \mathrm{H}), 1.80(\mathrm{~d}, J=14.5 \mathrm{~Hz}, 1 \mathrm{H}), 1.65(\operatorname{app~dt}, J=16.9,11.9 \mathrm{~Hz}, 1 \mathrm{H}), 1.45($ app tt, $J=12.8,4.4 \mathrm{~Hz}, 1 \mathrm{H})$, $1.23-0.97(\mathrm{~m}, 1 \mathrm{H})$.

${ }^{13}$ C-NMR (125 MHz, $\left.\mathrm{CDCl}_{3}\right): \delta=162.4,144.5,132.8,130.5,127.9,122.2,121.7,61.6,40.6,31.9,25.1,23.8$.

Accurate Mass (ESI): Calculated for $\mathrm{C}_{12} \mathrm{H}_{15} \mathrm{~N}_{2}[\mathrm{M}+\mathrm{H}]^{+}:$187.1230, Found: 187.1109, Spectral Accuracy: 98.3\%. 


\section{3-(Piperidin-2-yl)benzonitrile}

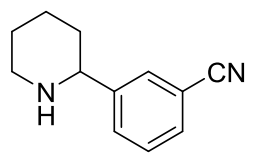

$( \pm)-8 d$

Following general procedure A, compound $( \pm)$-8d was obtained from piperidine $(99 \mu \mathrm{L}, 1 \mathrm{mmol})$, trifluoroacetophenone $(191.4 \mathrm{mg}, 1.2 \mathrm{mmol})$, the organometallic nucleophile $(1.5 \mathrm{mmol})$, and $\mathrm{BF}_{3} \cdot \mathrm{OEt}_{2}(148 \mu \mathrm{L}, 1.2$ mmol). Compound $( \pm)-8 d$ was obtained as a colorless oil in $52 \%$ yield $(96.8 \mathrm{mg})$.

The organometallic nucleophile was prepared as follows: A flame dried vial was charged with $i$-PrMgCl $\cdot \mathrm{LiCl}$ solution in THF $(1.33 \mathrm{~mL}, 1.13 \mathrm{M})$ and cooled in an ice bath followed by the addition of 3-bromobenzonitrile (273.3 $\mathrm{mg}, 1.5 \mathrm{mmol}, 1.5$ equiv) in $0.7 \mathrm{~mL}$ of dry THF. The $\mathrm{Br} / \mathrm{Mg}$ exchange was completed in 3 hours to yield a red colored solution.

\section{Characterization data:}

$\mathbf{R}_{\mathbf{f}}=0.37$ in EtOAc $/ \mathrm{MeOH} / i-\mathrm{PrNH}_{2}$ 90:9:1 v/v) $/ \mathrm{v}$

${ }^{1}$ H-NMR $\left(500 \mathrm{MHz}, \mathrm{CDCl}_{3}\right): \delta=7.67(\mathrm{dd}, J=1.7,1.0 \mathrm{~Hz}, 1 \mathrm{H}), 7.62-7.57(\mathrm{~m}, 1 \mathrm{H}), 7.51$ (app dt, $J=7.8,1.4 \mathrm{~Hz}$, 1H), 7.39 (app td, $J=7.8,1.2 \mathrm{~Hz}, 1 \mathrm{H}), 3.62(\mathrm{dd}, J=12.1,2.4 \mathrm{~Hz}, 1 \mathrm{H}), 3.18(\mathrm{dd}, J=12.1,2.4 \mathrm{~Hz}, 1 \mathrm{H}), 2.87-2.73$ (m, 1H), $2.04(\mathrm{~s}, 1 \mathrm{H}), 1.96-1.85(\mathrm{~m}, 1 \mathrm{H}), 1.83-1.73(\mathrm{~m}, 1 \mathrm{H}), 1.68-1.62(\mathrm{~m}, 1 \mathrm{H}), 1.59-1.37$ (comp, 3H).

${ }^{13}$ C-NMR (125 MHz, $\left.\mathrm{CDCl}_{3}\right): \delta=146.9,131.3,130.8,130.5,129.2,119.0,112.5,61.5,47.6,35.1,25.7,25.2$.

Accurate Mass (ESI): Calculated for $\mathrm{C}_{12} \mathrm{H}_{15} \mathrm{~N}_{2}[\mathrm{M}+\mathrm{H}]^{+}: 187.1230$, Found: 187.1120 , Spectral Accuracy: $98.2 \%$ 


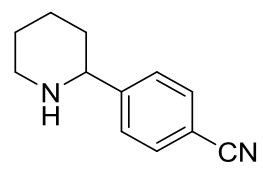

$( \pm)-8 e$

Following general procedure A, compound $( \pm)-8 \mathrm{e}$ was obtained from piperidine $(99 \mu \mathrm{L}, 1 \mathrm{mmol})$, trifluoroacetophenone $(191.4 \mathrm{mg}, 1.2 \mathrm{mmol})$, the organometallic nucleophile $(1.5 \mathrm{mmol})$, and $\mathrm{BF}_{3} \cdot \mathrm{OEt}_{2}(148 \mu \mathrm{L}, 1.2$ mmol). Compound $( \pm)-8 \mathbf{e}$ was obtained as a colorless oil in $33 \%$ yield $(61.3 \mathrm{mg})$.

The organometallic nucleophile was prepared as follows: A flame dried vial was charged with $i$ - $\mathrm{PrMgCl} \cdot \mathrm{LiCl}$ solution in THF $(1.33 \mathrm{~mL}, 1.13 \mathrm{M})$ and cooled in an ice bath followed by the addition of 4-bromobenzonitrile (273.3 $\mathrm{mg}, 1.5 \mathrm{mmol}, 1.5$ equiv) in $0.7 \mathrm{~mL}$ of dry THF. The $\mathrm{Br} / \mathrm{Mg}$ exchange was completed in 2 hours to yield a red colored solution.

\section{Characterization data:}

$\mathbf{R}_{\mathbf{f}}=0.22$ in $\mathrm{EtOAc} / \mathrm{MeOH} / i-\mathrm{PrNH}_{2}$ 90:9:1 v/v/v)

${ }^{1}$ H-NMR $\left(500 \mathrm{MHz}, \mathrm{CDCl}_{3}\right): \delta=7.58(\mathrm{~d}, J=8.3 \mathrm{~Hz}, 2 \mathrm{H}), 7.46(\mathrm{~d}, J=8.2 \mathrm{~Hz}, 2 \mathrm{H}), 3.64(\mathrm{dd}, J=10.7,2.6 \mathrm{~Hz}, 1 \mathrm{H})$, $3.23-3.12(\mathrm{~m}, 1 \mathrm{H}), 2.78($ app td, $J=11.7,2.8 \mathrm{~Hz}, 1 \mathrm{H}), 1.92-1.83(\mathrm{~m}, 1 \mathrm{H}), 1.82-1.72(\mathrm{comp}, 2 \mathrm{H}), 1.65$ (ddd, $J=$ 9.4, 3.0, $1.8 \mathrm{~Hz}, 1 \mathrm{H}), 1.59-1.35$ (comp, 3H).

${ }^{13}$ C-NMR $\left(125 \mathrm{MHz}, \mathrm{CDCl}_{3}\right): \delta=151.1,132.3,127.5,119.1,110.8,61.9,47.6,35.1,25.8,25.3$.

Accurate Mass (ESI): Calculated for $\mathrm{C}_{12} \mathrm{H}_{15} \mathrm{~N}_{2}[\mathrm{M}+\mathrm{H}]^{+}$: 187.1230, Found: 187.1087, Spectral Accuracy: 98.2\%. 


\section{3-(Azepan-2-yl)benzonitrile}

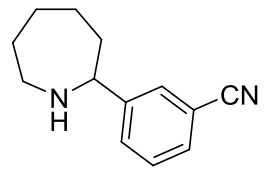

$( \pm)-8 f$

Following general procedure A, compound $( \pm)-\mathbf{8 f}$ was obtained from azepane $(113 \mu \mathrm{L}, 1 \mathrm{mmol})$, trifluoroacetophenone $(191.4 \mathrm{mg}, 1.2 \mathrm{mmol})$, the organometallic nucleophile $(1.5 \mathrm{mmol})$, and $\mathrm{BF}_{3} \cdot \mathrm{OEt}_{2}(148 \mu \mathrm{L}, 1.2$ $\mathrm{mmol})$. Compound ( \pm )-8f was obtained as a colorless oil in $34 \%$ yield $(68 \mathrm{mg})$.

The organometallic nucleophile was prepared as follows: A flame dried vial was charged with $i$-PrMgCl $\cdot \mathrm{LiCl}$ solution in THF $(1.33 \mathrm{~mL}, 1.13 \mathrm{M})$ and cooled in an ice bath followed by the addition of 3-bromobenzonitrile (273.3 $\mathrm{mg}, 1.5 \mathrm{mmol}, 1.5$ equiv) in $0.7 \mathrm{~mL}$ of dry THF. The $\mathrm{Br} / \mathrm{Mg}$ exchange was completed in 3 hours to yield a red colored solution.

\section{Characterization data:}

$\mathbf{R}_{\mathbf{f}}=0.25$ in EtOAc/MeOH$\left./ i-\mathrm{PrNH}_{2} 90: 9: 1 \mathrm{v} / \mathrm{v} / \mathrm{v}\right)$

${ }^{1}$ H-NMR $\left(500 \mathrm{MHz}, \mathrm{CDCl}_{3}\right): \delta=7.67(\mathrm{~d}, J=2.1 \mathrm{~Hz}, 1 \mathrm{H}), 7.58(\mathrm{dd}, J=8.0,1.7 \mathrm{~Hz}, 1 \mathrm{H}), 7.52-7.46(\mathrm{~m}, 1 \mathrm{H}), 7.38$ (app td, $J=7.8,1.7 \mathrm{~Hz}, 1 \mathrm{H}), 3.83($ app dt, $J=9.6,2.8 \mathrm{~Hz}, 1 \mathrm{H}), 3.19-2.94(\mathrm{~m}, 1 \mathrm{H}), 2.87$ (app ddt, $J=11.0,8.9,2.3$ $\mathrm{Hz}, 1 \mathrm{H}), 2.11$ (s, 1H), 1.99-1.92 (m, 1H), 1.88-1.48 (comp, 7H).

${ }^{13}$ C-NMR (125 MHz, $\left.\mathrm{CDCl}_{3}\right): \delta=148.5,131.2,130.5,130.3,129.2,119.1,112.4,63.4,47.7,38.9,31.1,27.3,26.0$.

Accurate Mass (ESI): Calculated for $\mathrm{C}_{13} \mathrm{H}_{17} \mathrm{~N}_{2}[\mathrm{M}+\mathrm{H}]^{+}:$201.1386, Found: 201.1313, Spectral Accuracy: 98.4\%. 


\section{1-Methyl-2-(trans-(6-phenylpiperidin-2-yl))-1H-indole}

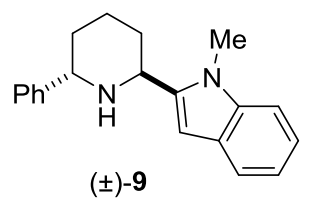

To a solution of $( \pm)-6 \mathbf{f}\left(107 \mathrm{mg}, 0.5 \mathrm{mmol}, 1.0\right.$ equiv) in anhydrous ether $(1 \mathrm{~mL})$ cooled at $-78{ }^{\circ} \mathrm{C}$ was added $n$-BuLi in hexanes $(0.5 \mathrm{mmol}, 1.0$ equiv) dropwise under the protection of nitrogen, and the resulting solution was stirred at the same temperature for $10 \mathrm{~min}$. To this was then added dropwise via cannula a solution of trifluoroacetophenone (104.4 $\mathrm{mg}, 0.6 \mathrm{mmol}, 1.2$ equiv) in anhydrous ether $(0.5 \mathrm{~mL})$, and the resulting mixture was allowed to continue stirring at $-78{ }^{\circ} \mathrm{C}$ for $10 \mathrm{~min}$ followed by the addition of the phenyl lithium solution in dibutyl ether $(0.75 \mathrm{mmol}, 1.5$ equiv) in one portion. The mixture was then warmed up to room temperature and stirred for another 2 hours before quenching with the addition of methanol $(0.25 \mathrm{~mL})$ at $0{ }^{\circ} \mathrm{C}$. The reaction mixture was diluted with ether $(20 \mathrm{~mL})$ and washed with water $(20 \mathrm{~mL})$. The aqueous layer was then extracted with ether $(3 \times 20 \mathrm{~mL})$ and the combined organic layers were washed with brine $(30 \mathrm{~mL})$ and dried over anhydrous $\mathrm{Na}_{2} \mathrm{SO}_{4}$. Solvent was removed under reduced pressure. The residue was purified by silica gel chromatography to give compound ( \pm )-9 as a colorless oil in $60 \%$ yield $(87 \mathrm{mg})$.

\section{Characterization data:}

$\mathbf{R}_{\mathbf{f}}=0.36$ in EtOAc/Hexanes 30:70 v/v)

${ }^{1}$ H-NMR (500 MHz, $\mathrm{CDCl}_{3}$ ): $\delta=7.59$ (app dt, $\left.J=7.9,1.0 \mathrm{~Hz}, 1 \mathrm{H}\right), 7.48-7.38$ (comp, 2H), 7.37-7.31 (comp, 2H), 7.30-7.26 (m, 1H), 7.26-7.21 (m, 1H), 7.18 (app ddq, $J=8.1,7.1,1.0 \mathrm{~Hz}, 1 \mathrm{H}), 7.09$ (app ddq, $J=7.9,7.1,1.0 \mathrm{~Hz}$, 1H), 6.65-6.48 (m, 1H), 4.41-4.27 (m, 1H), 4.06 (dd, $J=7.5,3.9 \mathrm{~Hz}, 1 \mathrm{H}), 3.72(\mathrm{~s}, 3 \mathrm{H}), 2.16-2.01$ (comp, 3H), $1.99-1.84$ (comp, 3H), 1.79 (s, 1H).

${ }^{13}$ C-NMR $\left(125 \mathrm{MHz}, \mathrm{CDCl}_{3}\right): \delta=144.4,142.5,137.7,128.5,127.5,127.1,126.9,121.3,120.3,119.5,109.0,100.0$, $54.6,48.7,32.2,30.3,29.8,21.2$.

Accurate Mass (ESI): Calculated for $\mathrm{C}_{20} \mathrm{H}_{23} \mathrm{~N}_{2}[\mathrm{M}+\mathrm{H}]^{+}:$291.1856, Found: 291.1838, Spectral Accuracy: 99.5\%. 


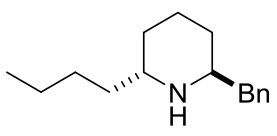

$( \pm)-10$

To a solution of $( \pm)-7 \mathbf{d}\left(87.5 \mathrm{mg}, 0.5 \mathrm{mmol}, 1.0\right.$ equiv) in anhydrous ether $(1 \mathrm{~mL})$ cooled at $-78{ }^{\circ} \mathrm{C}$ was added $n$ $\mathrm{BuLi}$ in hexanes $(0.5 \mathrm{mmol}, 1.0$ equiv) dropwise under the protection of nitrogen, and the resulting solution was stirred at the same temperature for $10 \mathrm{~min}$. To this was then added dropwise via cannula a solution of trifluoroacetophenone $(104.4 \mathrm{mg}, 0.6 \mathrm{mmol}, 1.2$ equiv) in anhydrous ether $(0.5 \mathrm{~mL})$, and the resulting mixture was stirred at $-78^{\circ} \mathrm{C}$ for $10 \mathrm{~min}$ followed by the addition of $n$-BuLi in hexanes $(0.75 \mathrm{mmol}, 1.5$ equiv $)$ in one portion. The mixture was then stirred at the same temperature for $90 \mathrm{~min}$ and then warmed up to room temperature and stirred for another $2 \mathrm{~h}$ before quenching with the addition of methanol $(0.25 \mathrm{~mL})$ at $0{ }^{\circ} \mathrm{C}$. The reaction mixture was diluted with ether $(20 \mathrm{~mL})$ and washed with water $(20 \mathrm{~mL})$. The aqueous layer was then extracted with ether $(3 \mathrm{x} 20$ $\mathrm{mL})$ and the combined organic layers were washed with brine $(30 \mathrm{~mL})$ and dried over anhydrous $\mathrm{Na}_{2} \mathrm{SO}_{4} . \mathrm{Solvent}$ was removed under reduced pressure. The residue was purified by silica gel chromatography to give compound $( \pm$ )10 as a colorless oil in $64 \%$ yield $(74 \mathrm{mg})$.

\section{Characterization data:}

$\mathbf{R}_{\mathbf{f}}=0.28$ in EtOAc/MeOH$/ i-\mathrm{PrNH}_{2}$ 90:9:1 v/v/v)

${ }^{1} \mathbf{H}-\mathbf{N M R}\left(500 \mathrm{MHz} \mathrm{CDCl}_{3}\right.$ ): $\delta=7.36-7.27$ (comp, 2H), 7.25-7.14 (comp, 3H), 3.09 (app ddt, $J=9.5,6.0,3.0 \mathrm{~Hz}$, 1H), 2.87 (app dp, $J=10.1,3.8,3.3 \mathrm{~Hz}, 1 \mathrm{H}), 2.82-2.66$ (comp, 2H), 1.78-1.52 (comp, 5H), 1.46-1.30 (comp, 2H), 1.30-1.16 (comp, 4H), 1.13-0.97 (comp, 2H), 0.83 (t, $J=7.3 \mathrm{~Hz}, 3 \mathrm{H})$.

${ }^{13}$ C-NMR $\left(125 \mathrm{MHz}, \mathrm{CDCl}_{3}\right): \delta=139.9,129.2,128.5,126.2,52.5,50.7,40.9,34.0,31.2,31.1,28.4,22.9,19.9$, 14.2 .

Accurate Mass (ESI): Calculated for $\mathrm{C}_{16} \mathrm{H}_{26} \mathrm{~N}[\mathrm{M}+\mathrm{H}]^{+}:$232.2060, Found: 232.2063, Spectral Accuracy: $98.6 \%$. 
2D-NMR Analysis for Compound ( \pm )-6i, Selected Interactions

GcosY

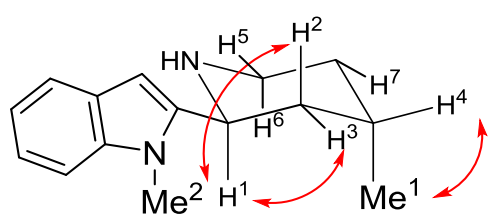

NOESY

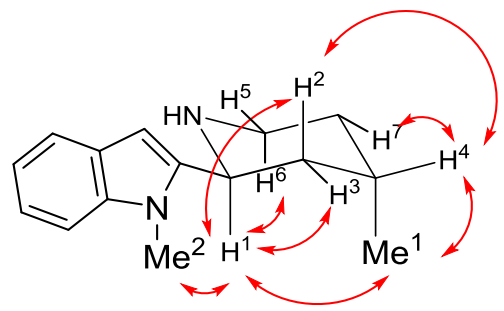

\section{${ }^{1} \mathrm{H}$ NMR shifts}

\begin{tabular}{|c|c|}
\hline Protons & Chemical shifts (ppm) \\
\hline $\mathrm{H}^{1}$ & 4.23 \\
\hline $\mathrm{H}^{2}$ & 2.08 \\
\hline $\mathrm{H}^{3}$ & $1.95-1.65$ \\
\hline $\mathrm{H}^{4}$ & $2.32-2.19$ \\
\hline $\mathrm{H}^{5}$ & 2.93 \\
\hline $\mathrm{H}^{6}$ & 3.01 \\
\hline $\mathrm{H}^{7}$ & 1.41 \\
\hline $\mathrm{Me}^{1}$ & 1.16 \\
\hline $\mathrm{Me}^{2}$ & 3.79 \\
\hline
\end{tabular}




\section{References:}

1 Suffert, J. Simple Direct Titration of Organolithium Reagents Using $N$-Pivaloyl-o-toluidine and/or $N$ Pivaloyl -o-benzylaniline. J. Org. Chem. 1989, 54, 510.

2 a) Kind, T.; Fiehn, O. Seven Golden Rules for Heuristic Filtering of Molecular Formulas Obtained by Accurate Mass Spectrometry. BMC Bioinformatics 2007, 8, 105. b) Wang, Y.; Gu, M. The concept of spectral accuracy for MS. Anal. Chem. 2010, 82, 7055.

3 Chen, W.; Ma, L.; Paul, A.; Seidel, D. Direct $\alpha-\mathrm{C}-\mathrm{H}$ bond functionalization of unprotected cyclic amines. Nat. Chem. 2018, 10, 165.

4 Richers, J.; Pçthig, A.; Herdtweck, E.; Sippel, C.; Hausch, F.; Tiefenbacher, K. Synthesis and Neurotrophic Activity Studies of Illicium Sesquiterpene Natural Product Analogues. Chem. Eur. J. 2017, 23, 3178 . 


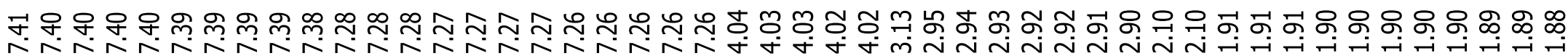

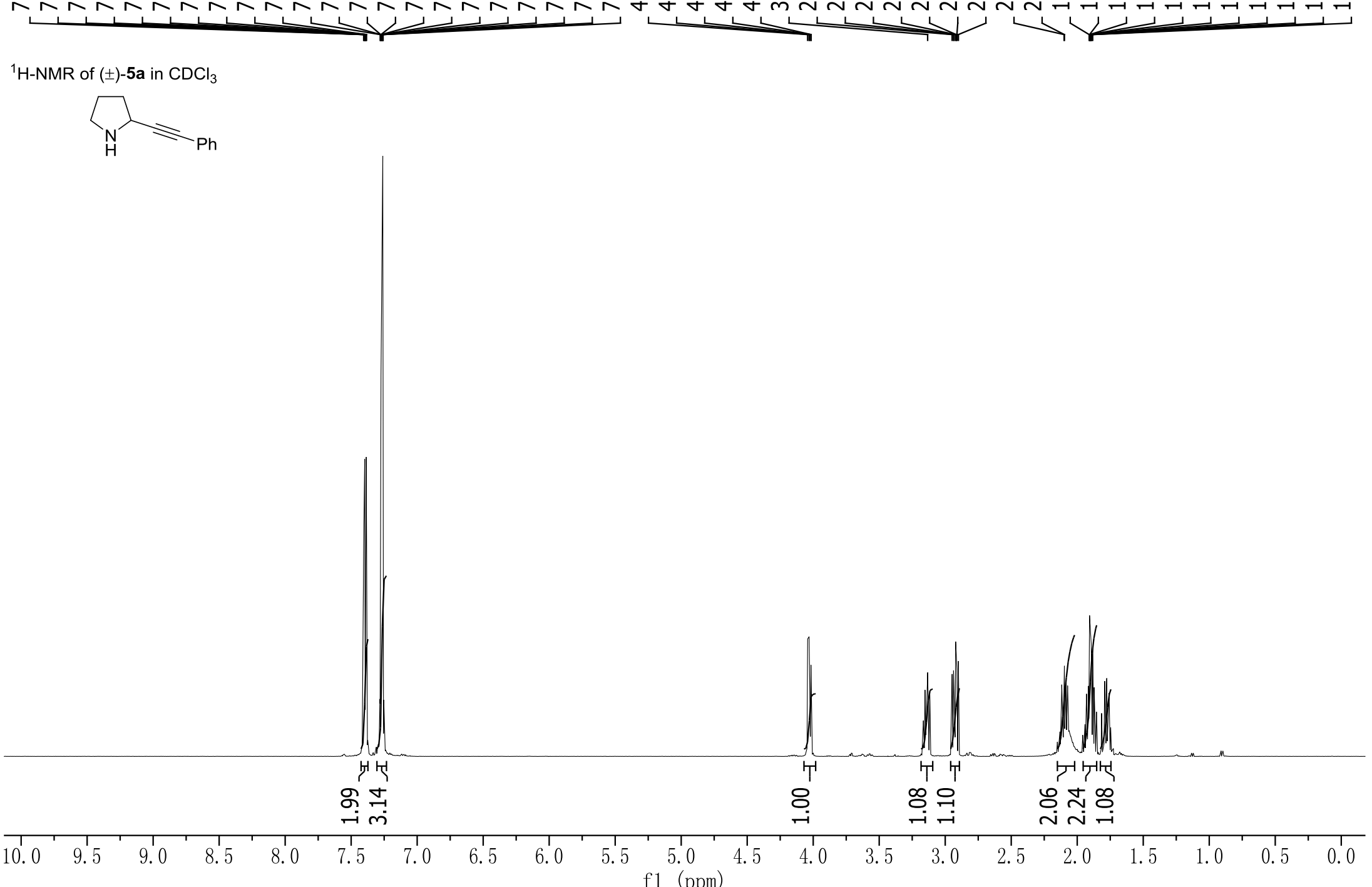




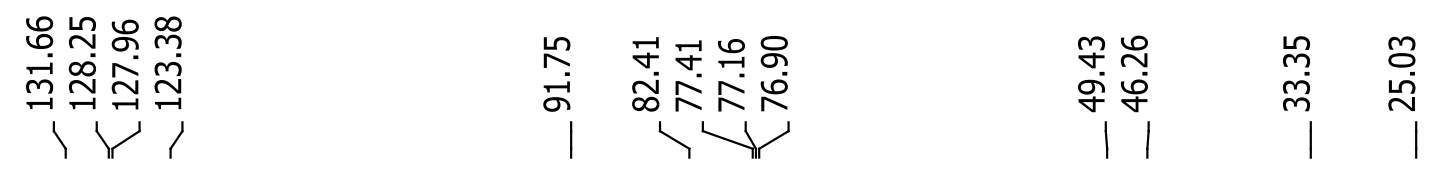

${ }^{13} \mathrm{C}-\mathrm{NMR}$ of $( \pm)-5 \mathrm{a}$ in $\mathrm{CDCl}_{3}$

$\overbrace{\mathrm{H}} \succ_{\mathrm{Ph}}$
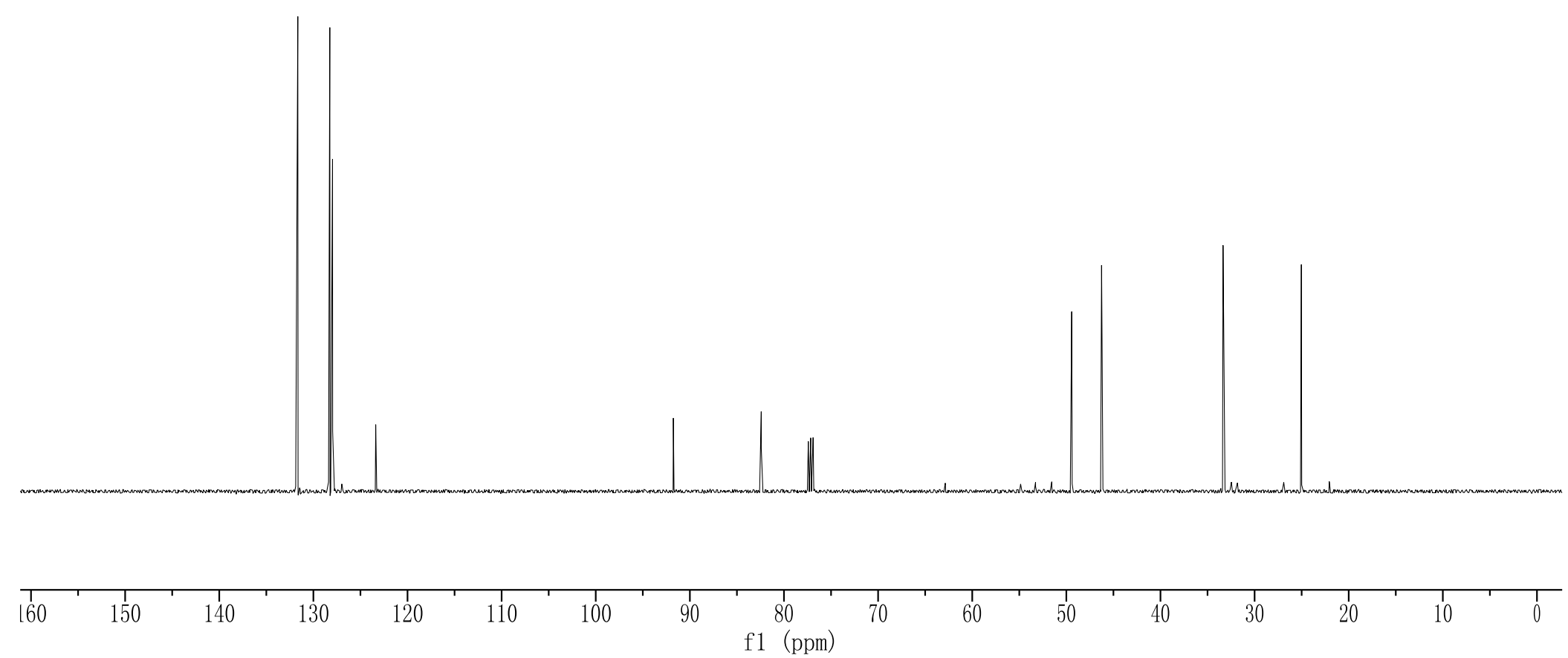


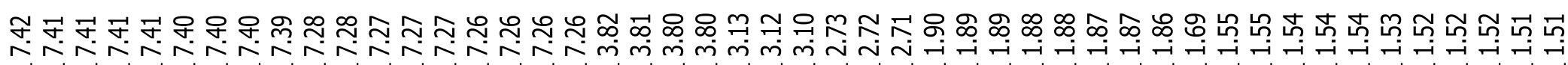
${ }^{1} \mathrm{H}-\mathrm{NMR}$ of $( \pm)-5 \mathbf{b}$ in $\mathrm{CDCl}_{3}$
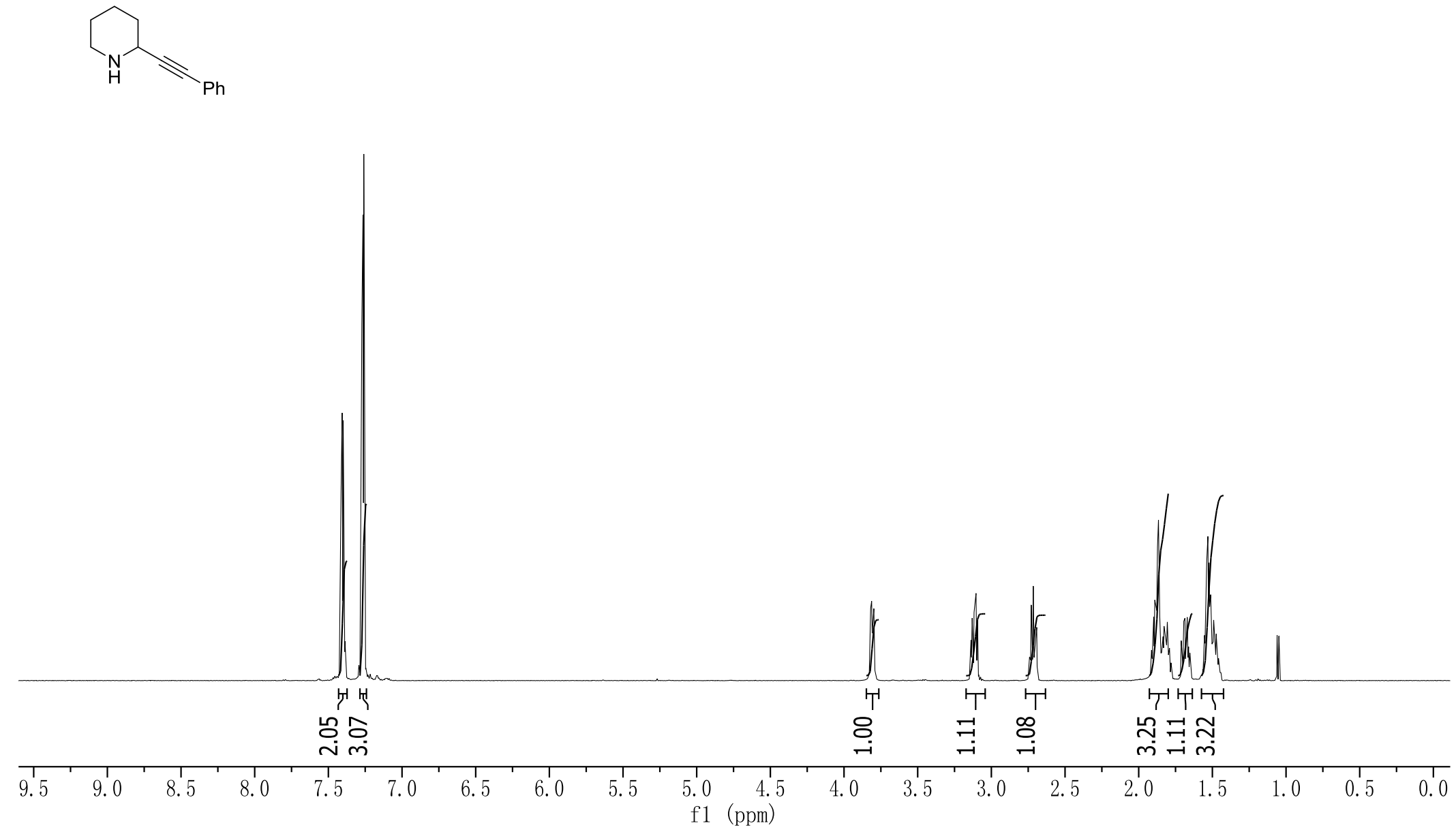

ํํำ

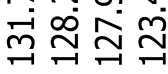
|W।
至

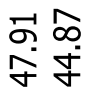
용ำ
南
लंi

${ }^{13} \mathrm{C}$-NMR of $( \pm)-5 \mathbf{b}$ in $\mathrm{CDCl}_{3}$<smiles>C(#CC1CCCCN1)c1ccccc1</smiles>

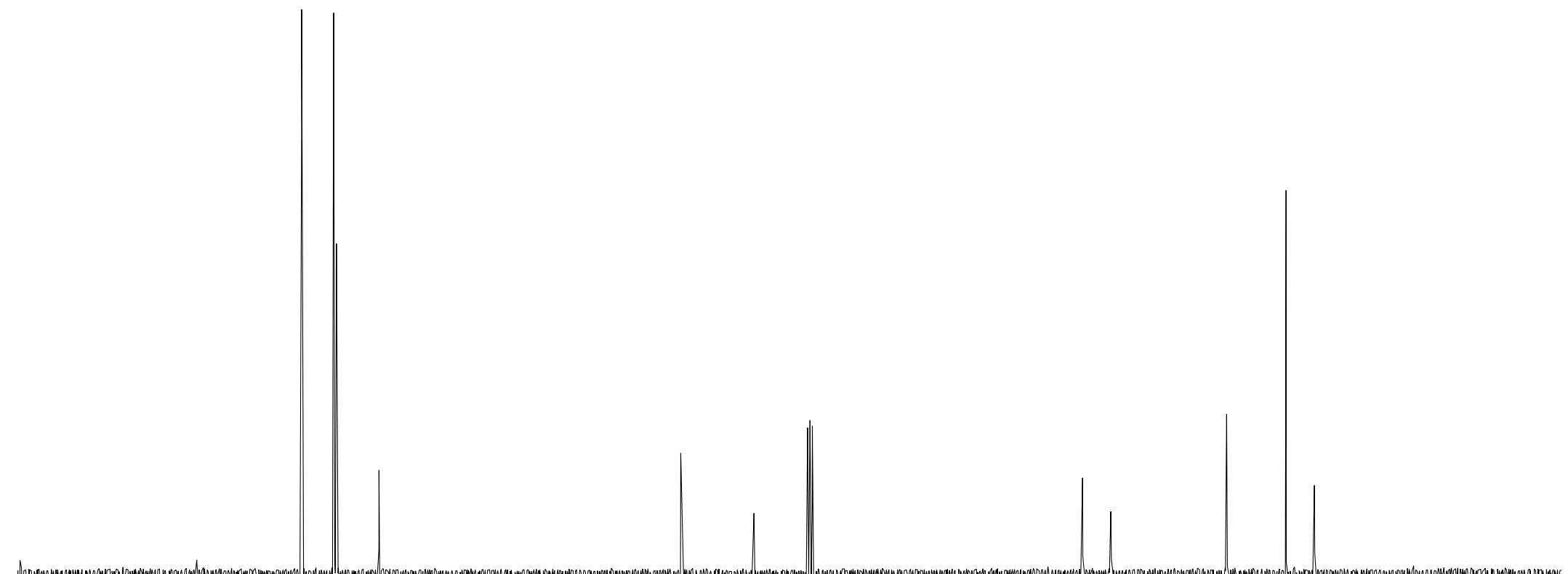

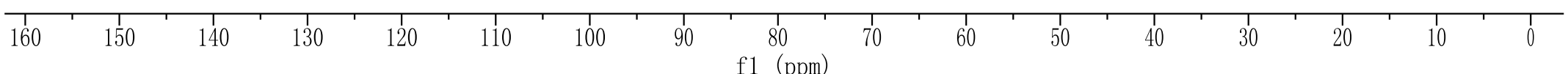




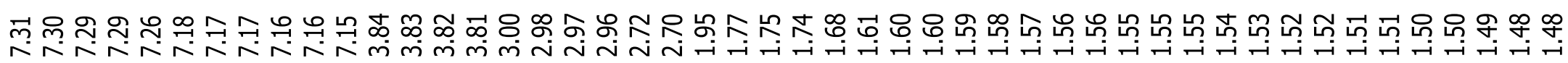
$\longrightarrow$

${ }^{1} \mathrm{H}-\mathrm{NMR}$ of $( \pm)-5 \mathrm{c}$ in $\mathrm{CDCl}_{3}$
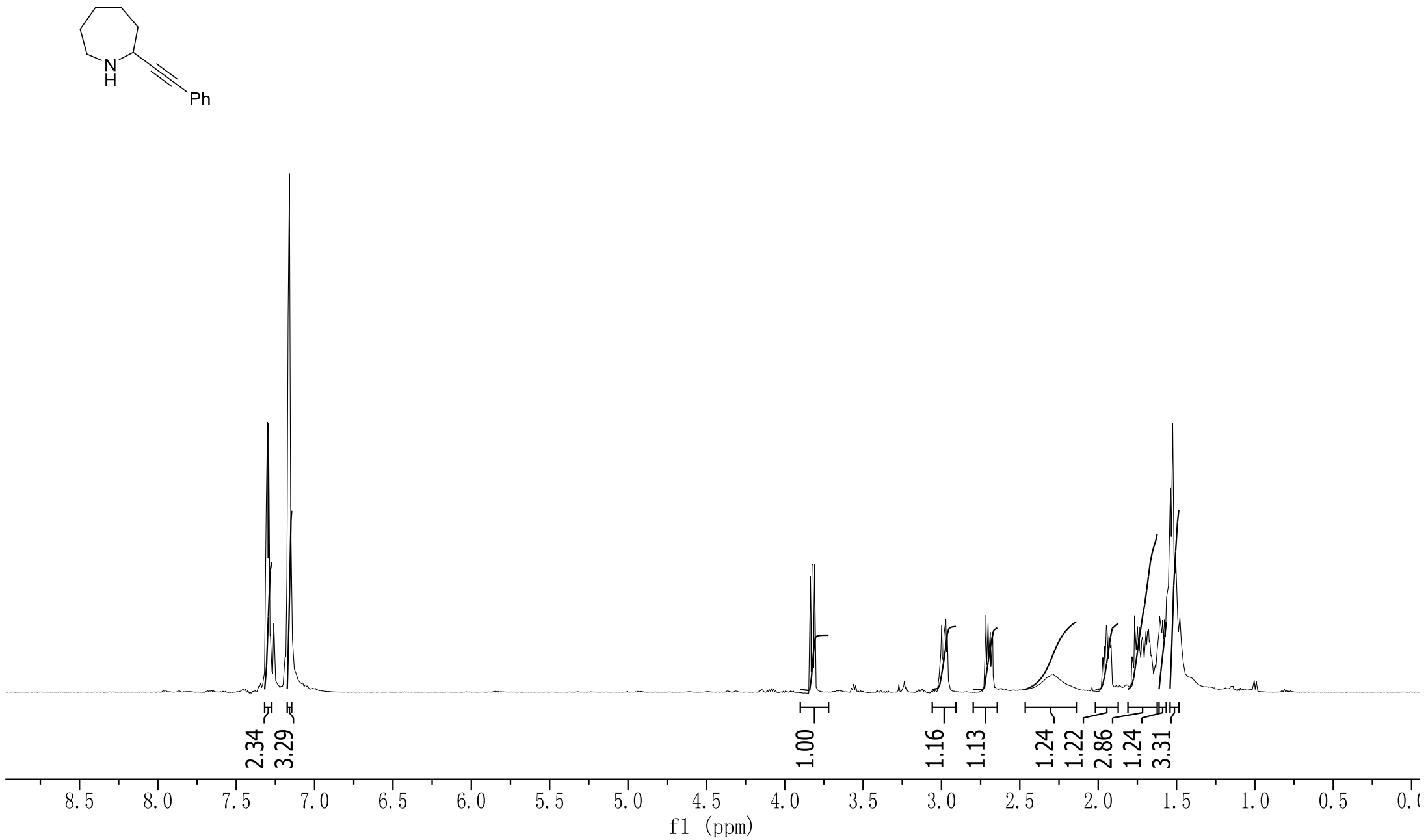


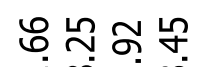

ตํับำ

1

${ }^{13} \mathrm{C}-\mathrm{NMR}$ of $( \pm)-5 \mathrm{c}$ in $\mathrm{CDCl}_{3}$
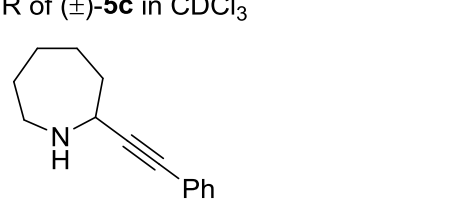

,

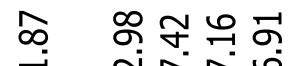

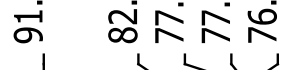

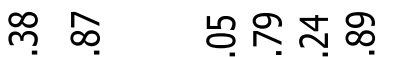

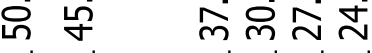

। ।

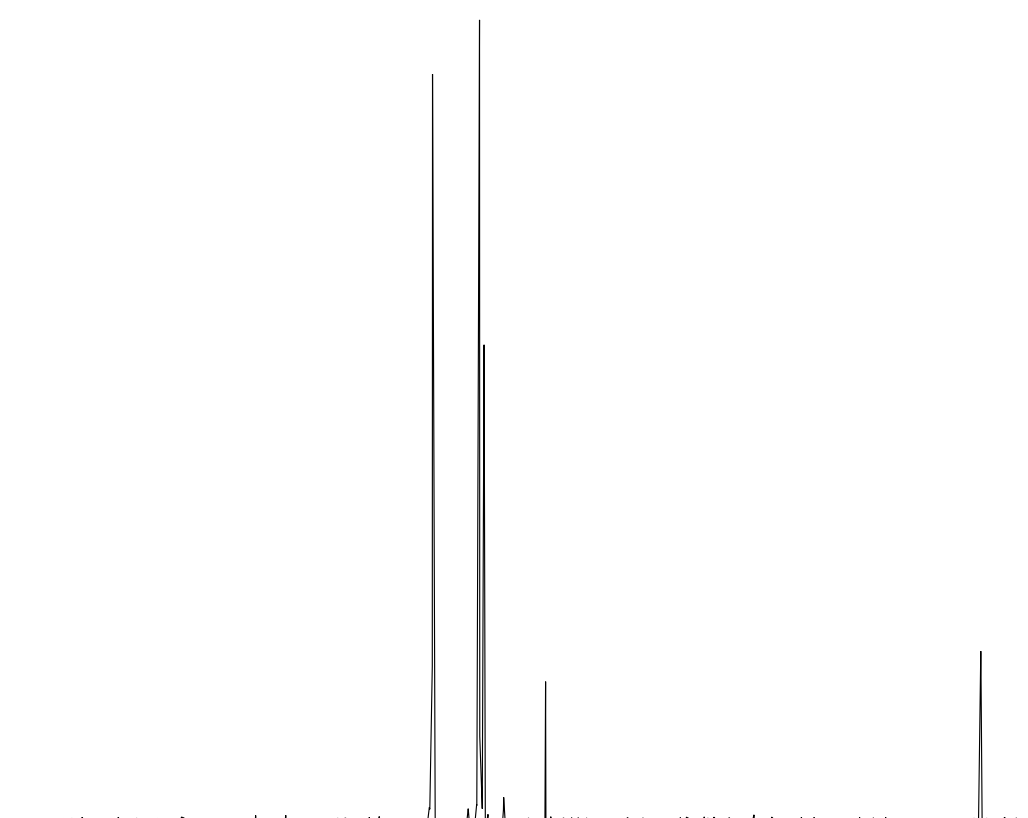

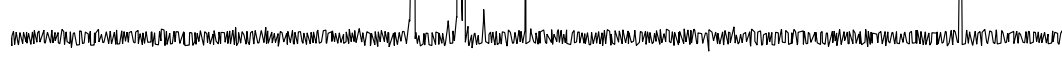
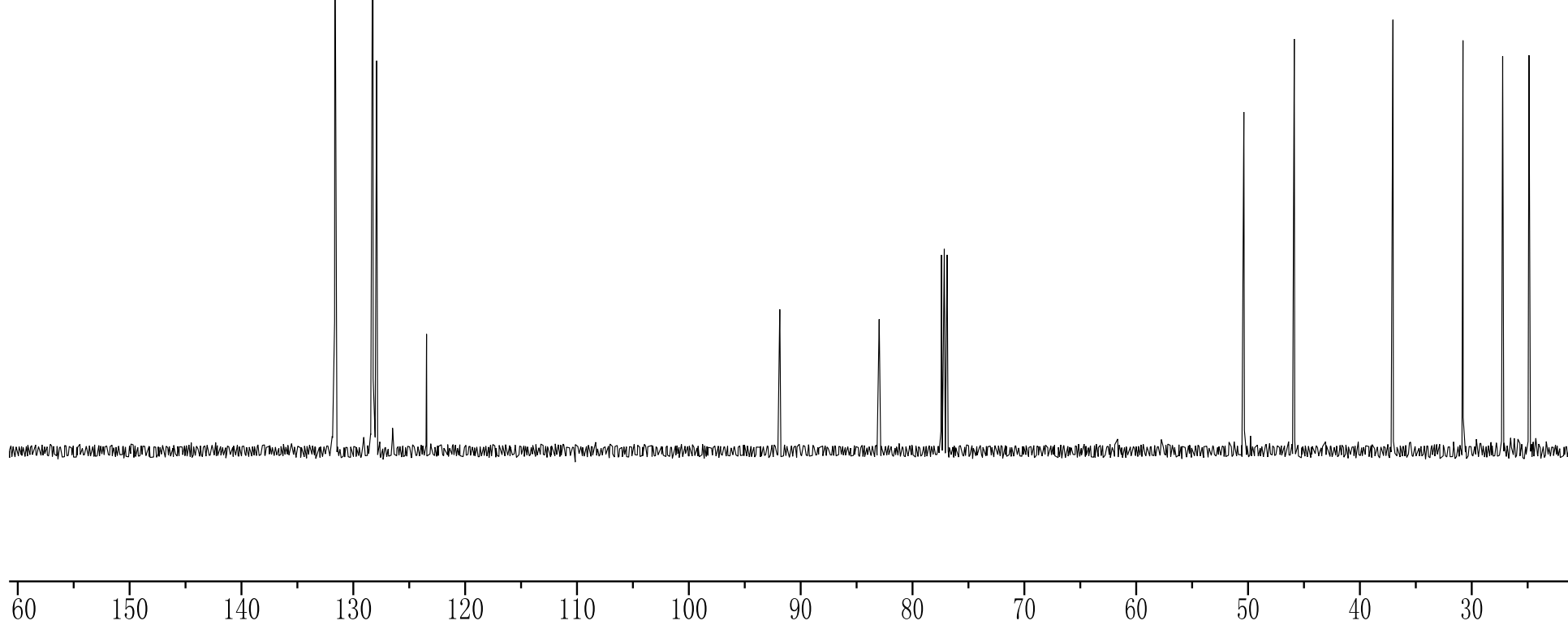

\begin{tabular}{lllll}
\hline 60 & 150 & 140 & 130 & 120
\end{tabular}

$120 \quad 110$

$10 \quad 100$

90

$\mathrm{f1}$

1 (ppm) 


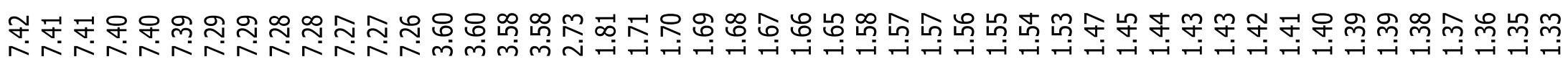
$\longrightarrow$

${ }^{1} \mathrm{H}-\mathrm{NMR}$ of $( \pm)-\mathbf{5 d}$ in $\mathrm{CDCl}_{3}$
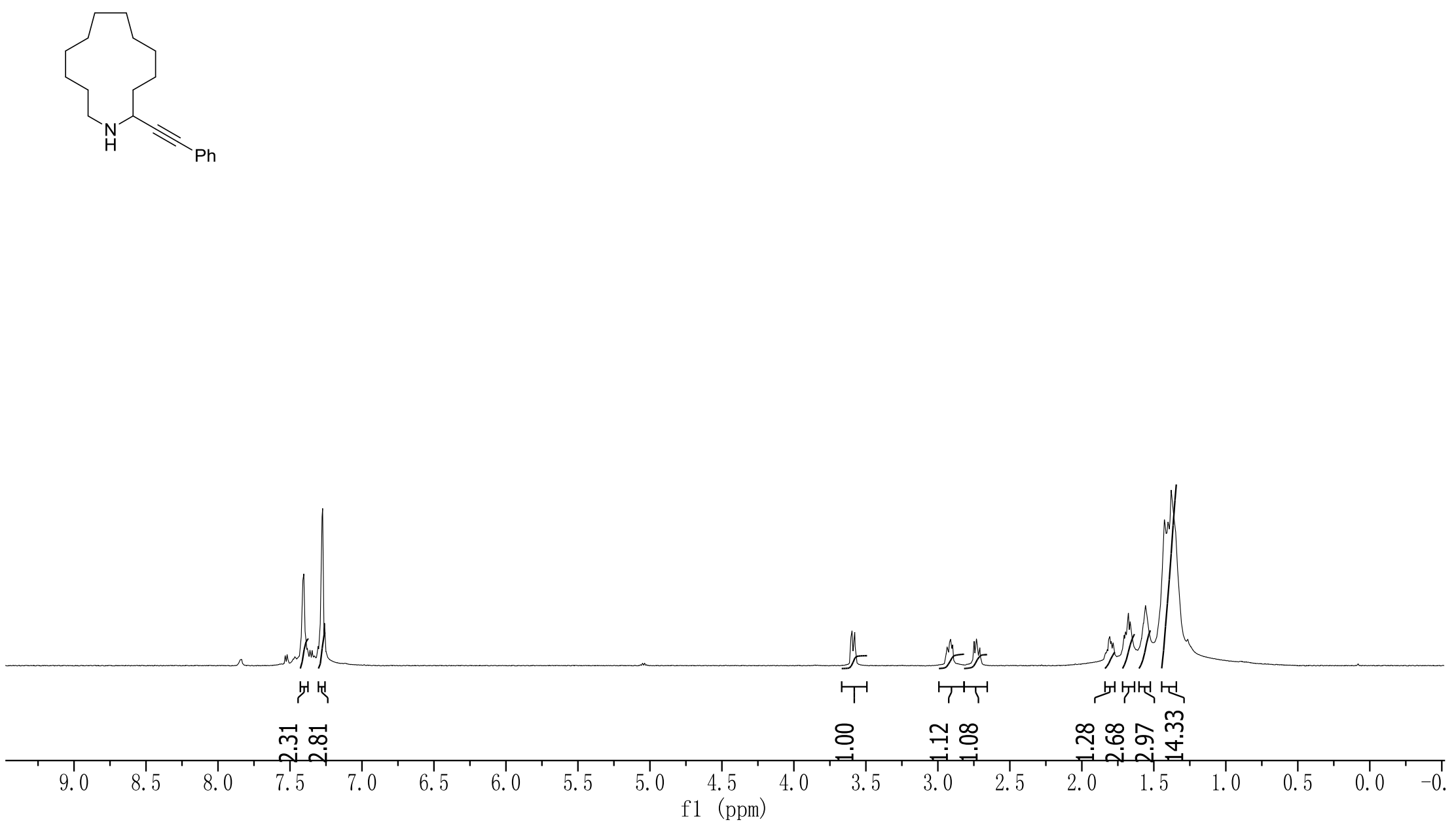


\begin{tabular}{|c|c|c|c|c|}
\hline 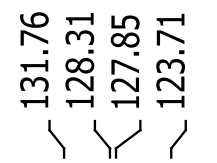 & $\stackrel{\substack{q \\
\text { d }}}{1}$ & 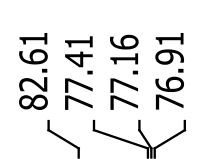 & 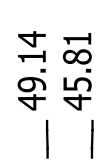 & 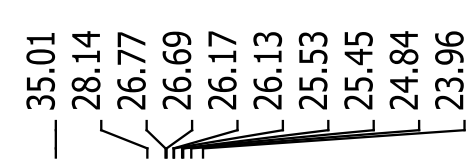 \\
\hline
\end{tabular}

${ }^{13} \mathrm{C}-\mathrm{NMR}$ of $( \pm)-\mathbf{5 d}$ in $\mathrm{CDCl}_{3}$
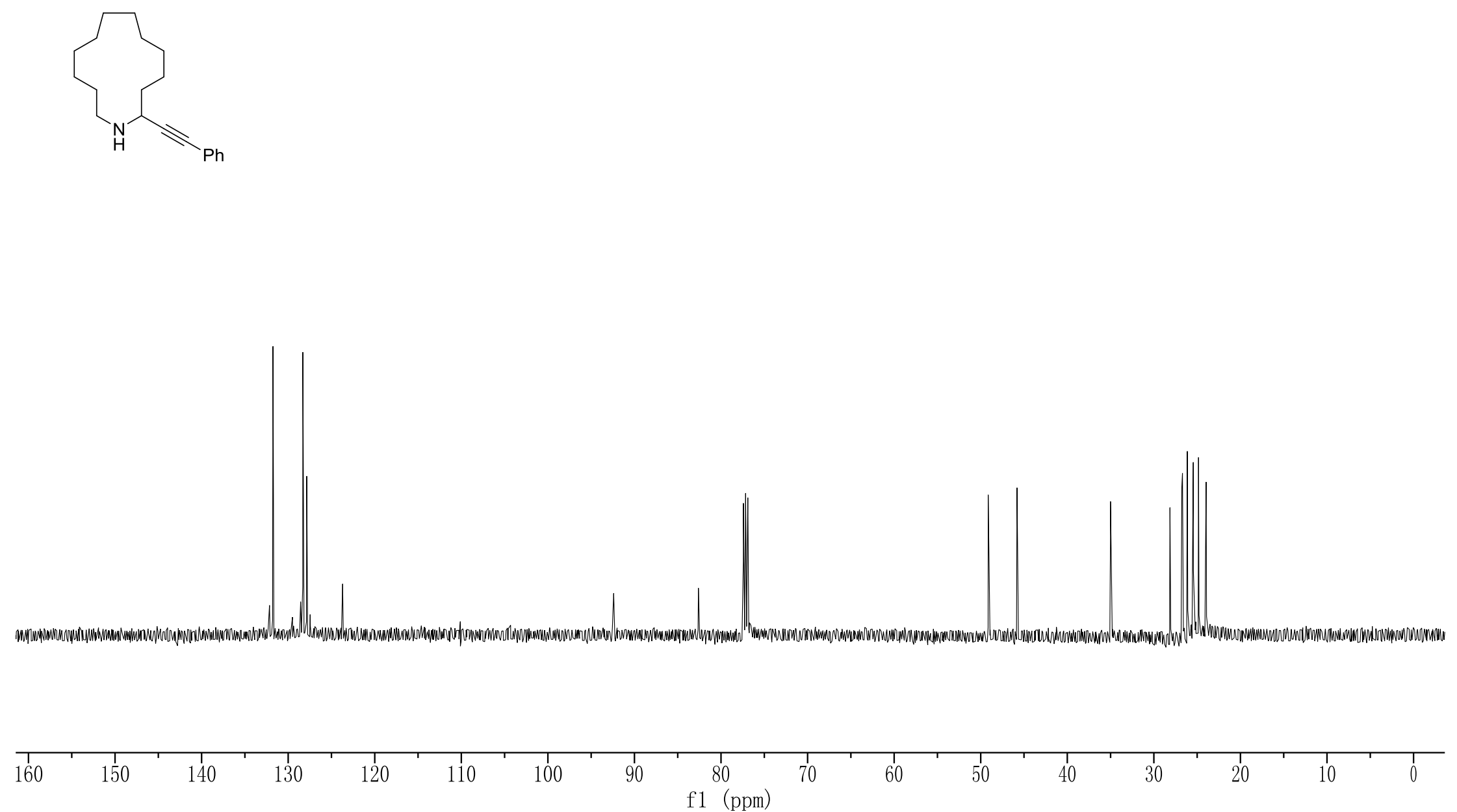


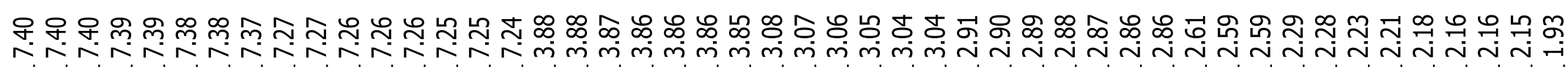

${ }^{1} \mathrm{H}-\mathrm{NMR}$ of $( \pm)-5 \mathbf{e}$ in $\mathrm{CDCl}_{3}$
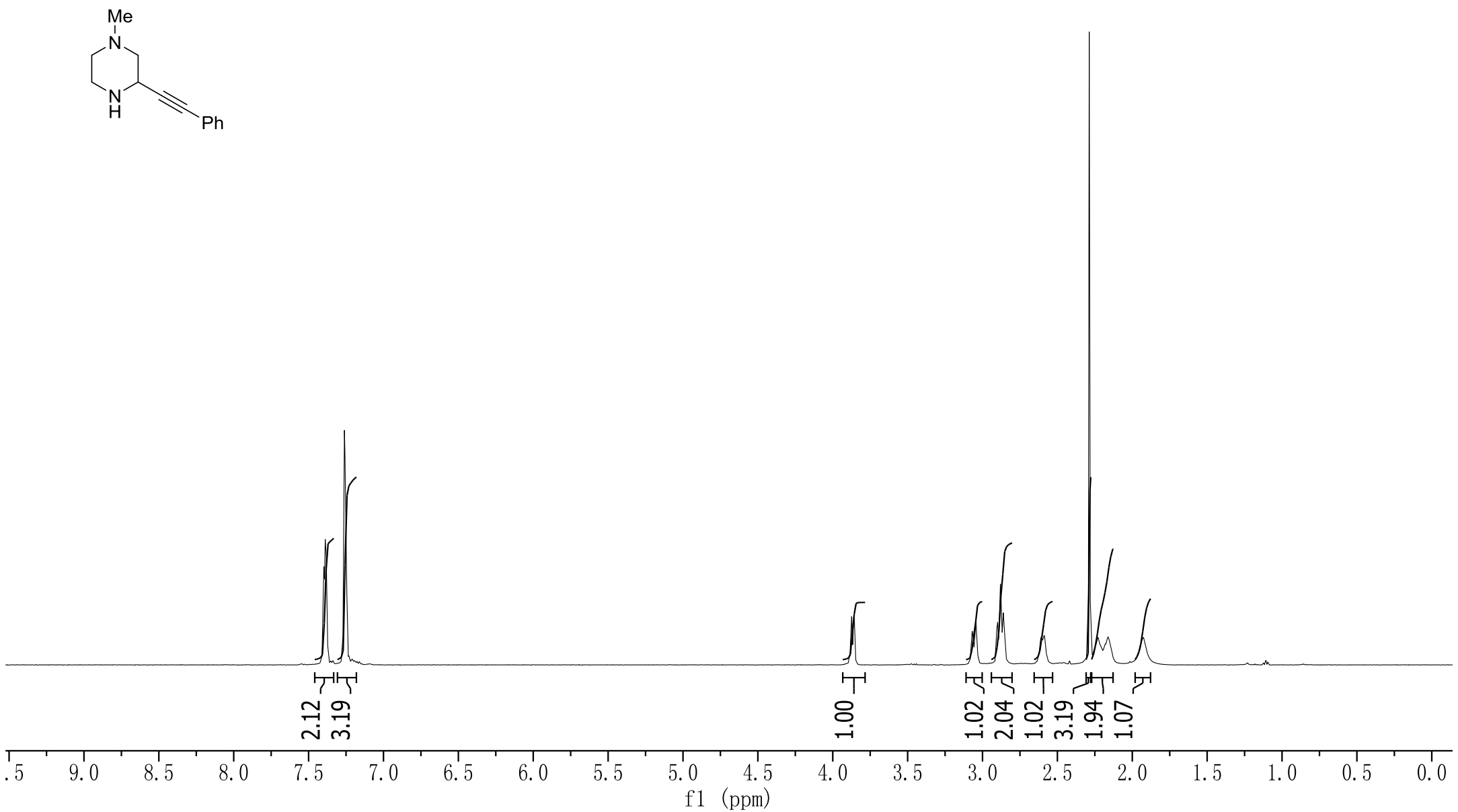


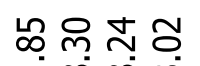

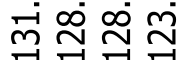

${ }^{13} \mathrm{C}-\mathrm{NMR}$ of $( \pm)-5 \mathbf{e}$ in $\mathrm{CDCl}_{3}$

11

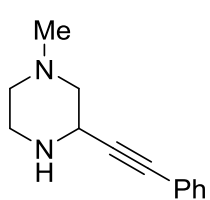

$\mid$

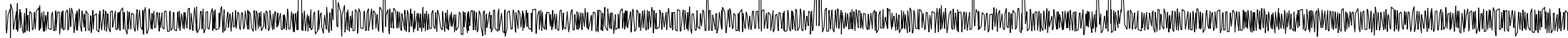
ஜ ํㅜ눙

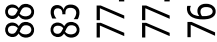
$1>$ Y

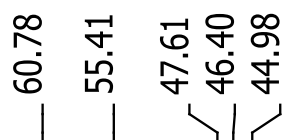

160

150

140

130

120

110

100

90

80

$70 \quad 60$




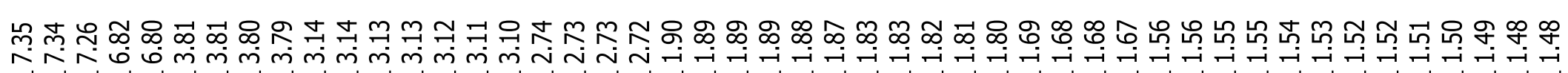
L ${ }^{1} \mathrm{H}-\mathrm{NMR}$ of $( \pm)-\mathbf{5 f}$ in $\mathrm{CDCl}_{3}$
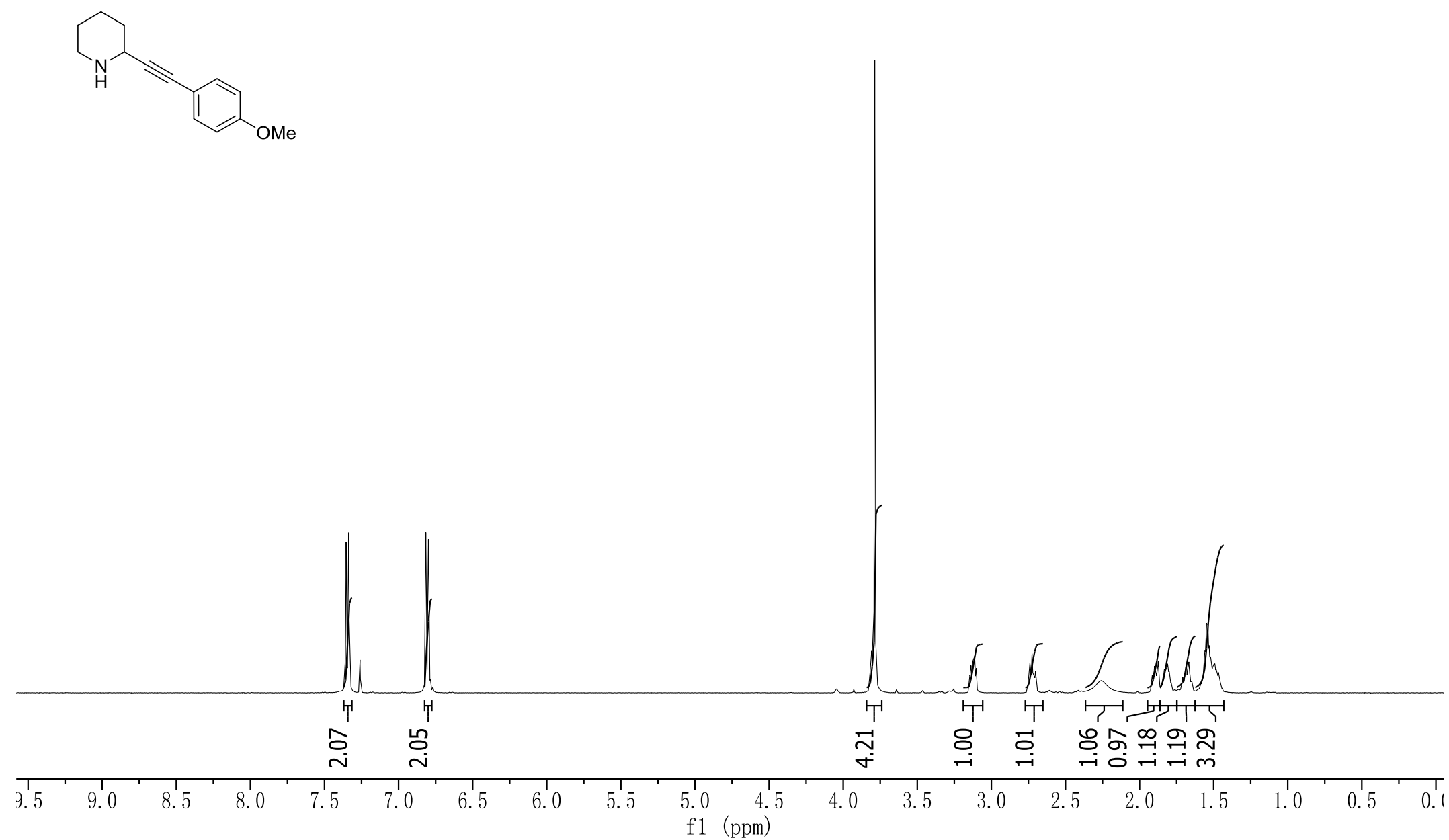


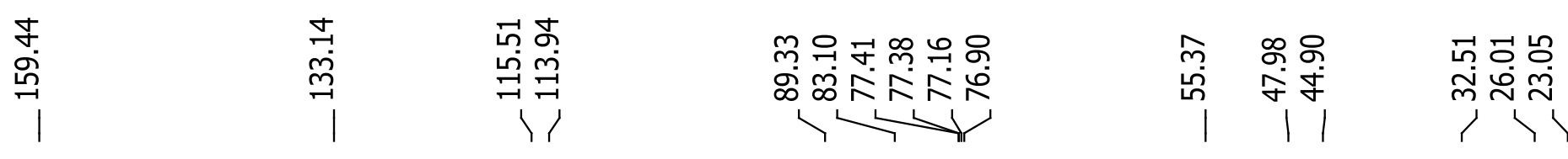

${ }^{13} \mathrm{C}-\mathrm{NMR}$ of $( \pm)-5 \mathrm{f}$ in $\mathrm{CDCl}_{3}$
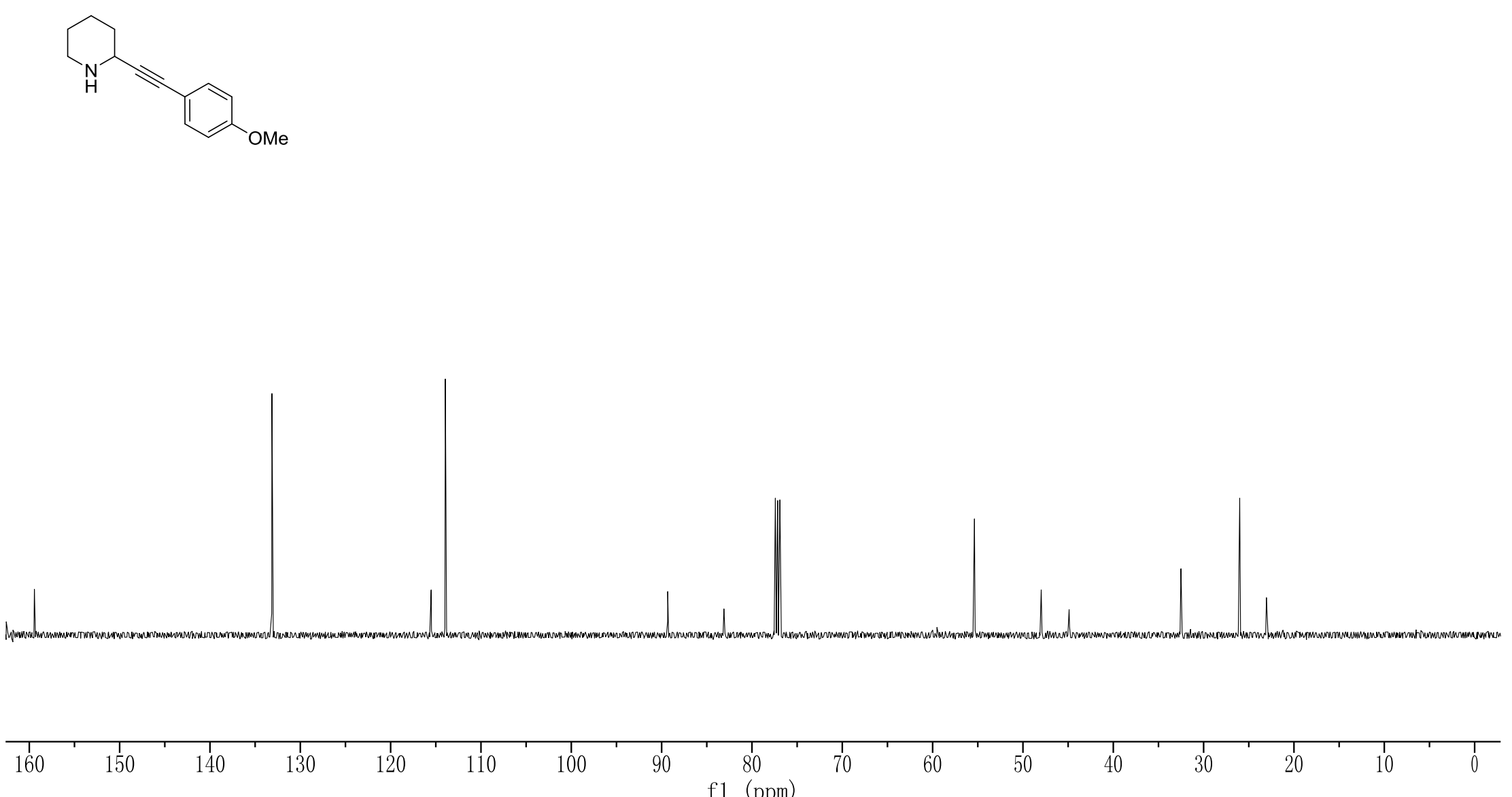


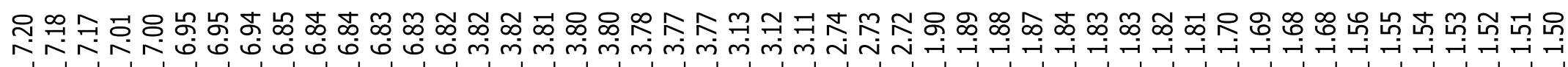

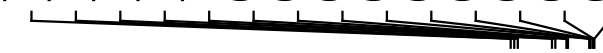

${ }^{1} \mathrm{H}-\mathrm{NMR}$ of $( \pm)-\mathbf{5 g}$ in $\mathrm{CDCl}_{3}$

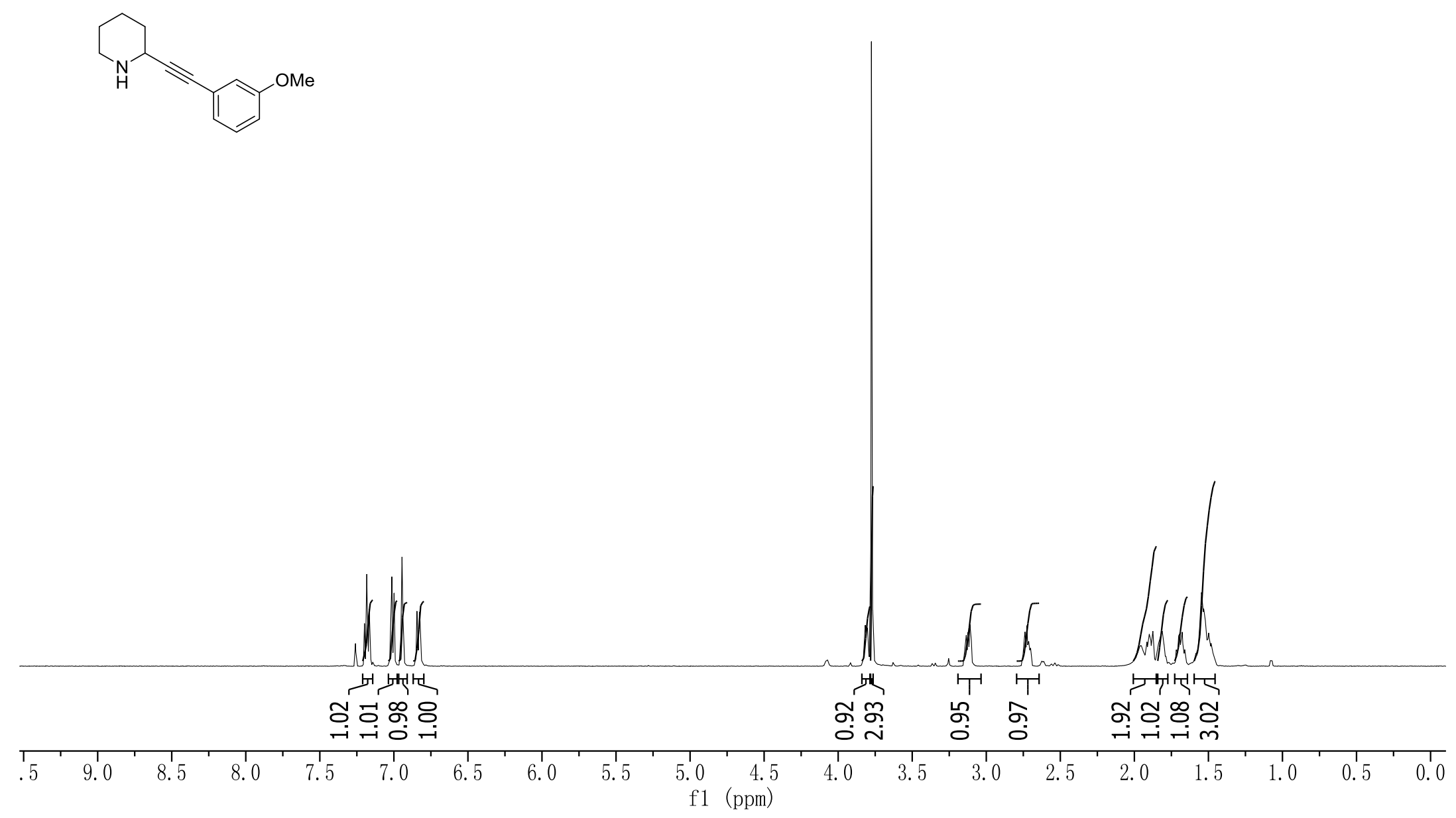


חి

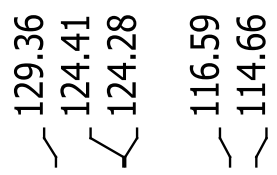

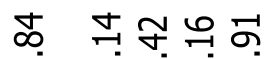

\&ं mNㅗㅇ

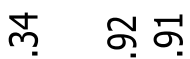

Ұषํㅇ

กิ ₹

กิ ข

1

$1 \backslash 1$

${ }^{13} \mathrm{C}-\mathrm{NMR}$ of $( \pm)-\mathbf{5 g}$ in $\mathrm{CDCl}_{3}$

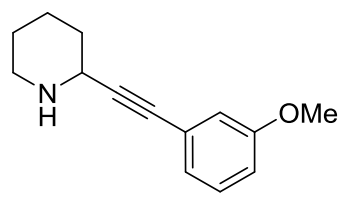

$|+|||||||||$

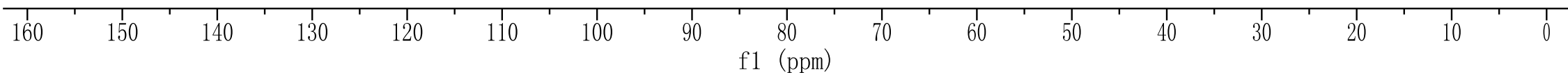




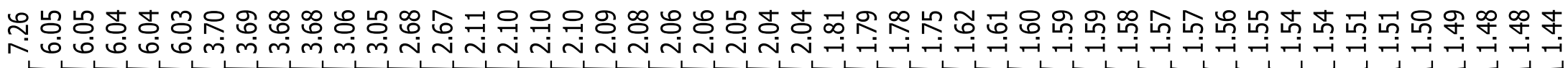

${ }^{1} \mathrm{H}-\mathrm{NMR}$ of $( \pm)-5 \mathbf{h}$ in $\mathrm{CDCl}_{3}$
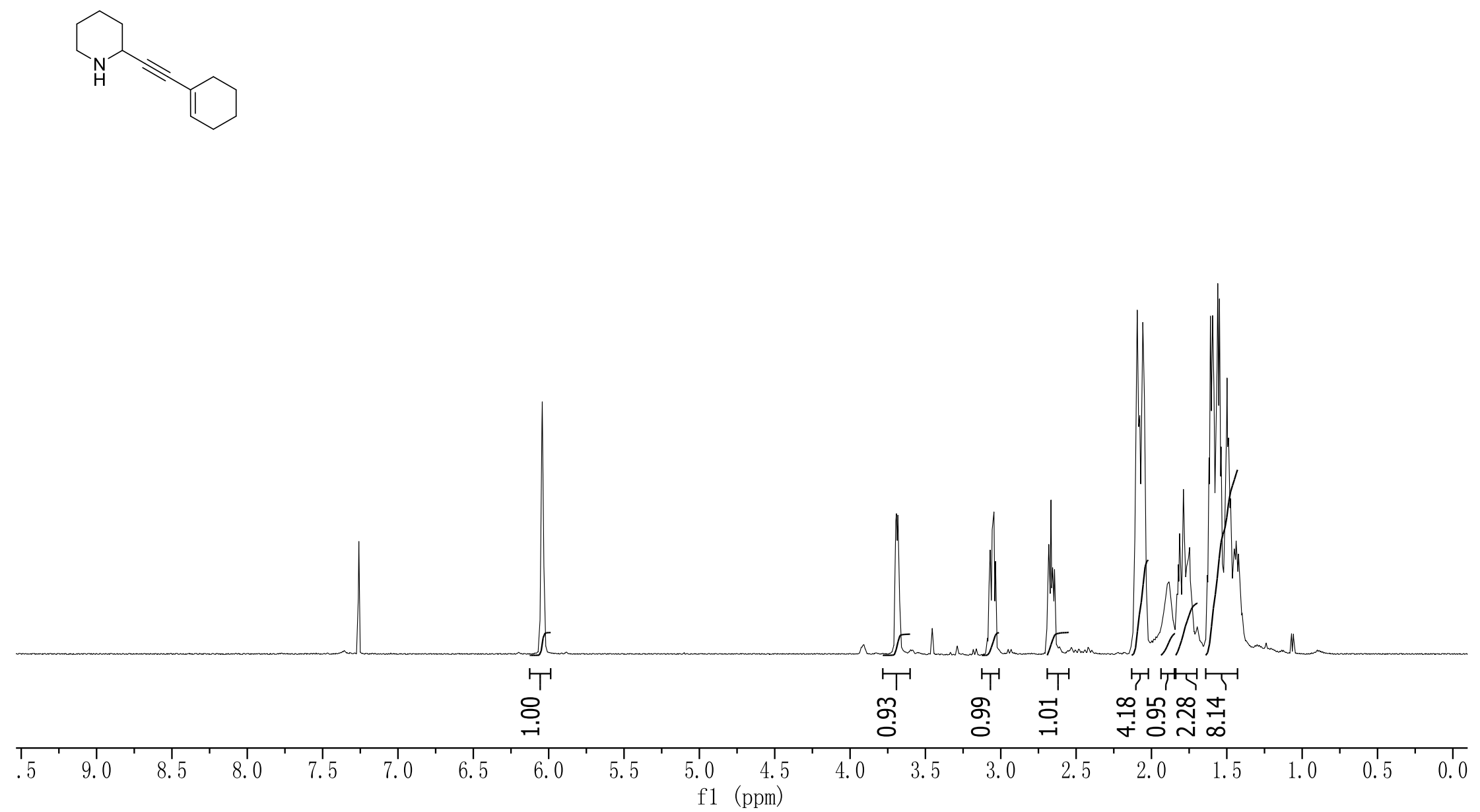


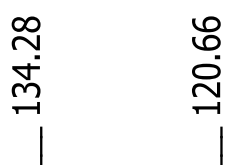

$\stackrel{\infty}{\rightarrow ㅁ ำ ~}$

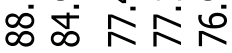

@

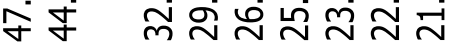

$+1$

${ }^{13} \mathrm{C}-\mathrm{NMR}$ of $( \pm)-5 \mathrm{~h}$ in $\mathrm{CDCl}_{3}$

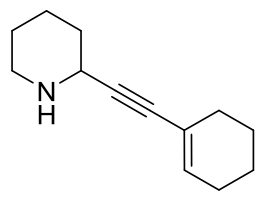

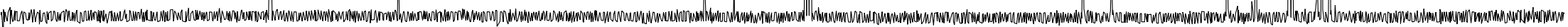

\begin{tabular}{lllllllllllllllll}
\hline 1 & 1 \\
160 & 150 & 140 & 130 & 120 & 110 & 100 & 90 & 80 & 10 & 60 & 50 & 40 & 30 & 20 & 10 & 0
\end{tabular}




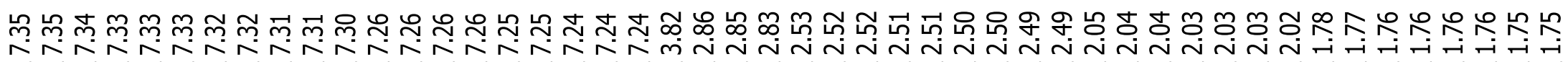

${ }^{1} \mathrm{H}-\mathrm{NMR}$ of $( \pm)-\mathbf{5 i}$ in $\mathrm{CDCl}_{3}$<smiles>C(#CC1CCCN1)Cc1ccccc1</smiles>

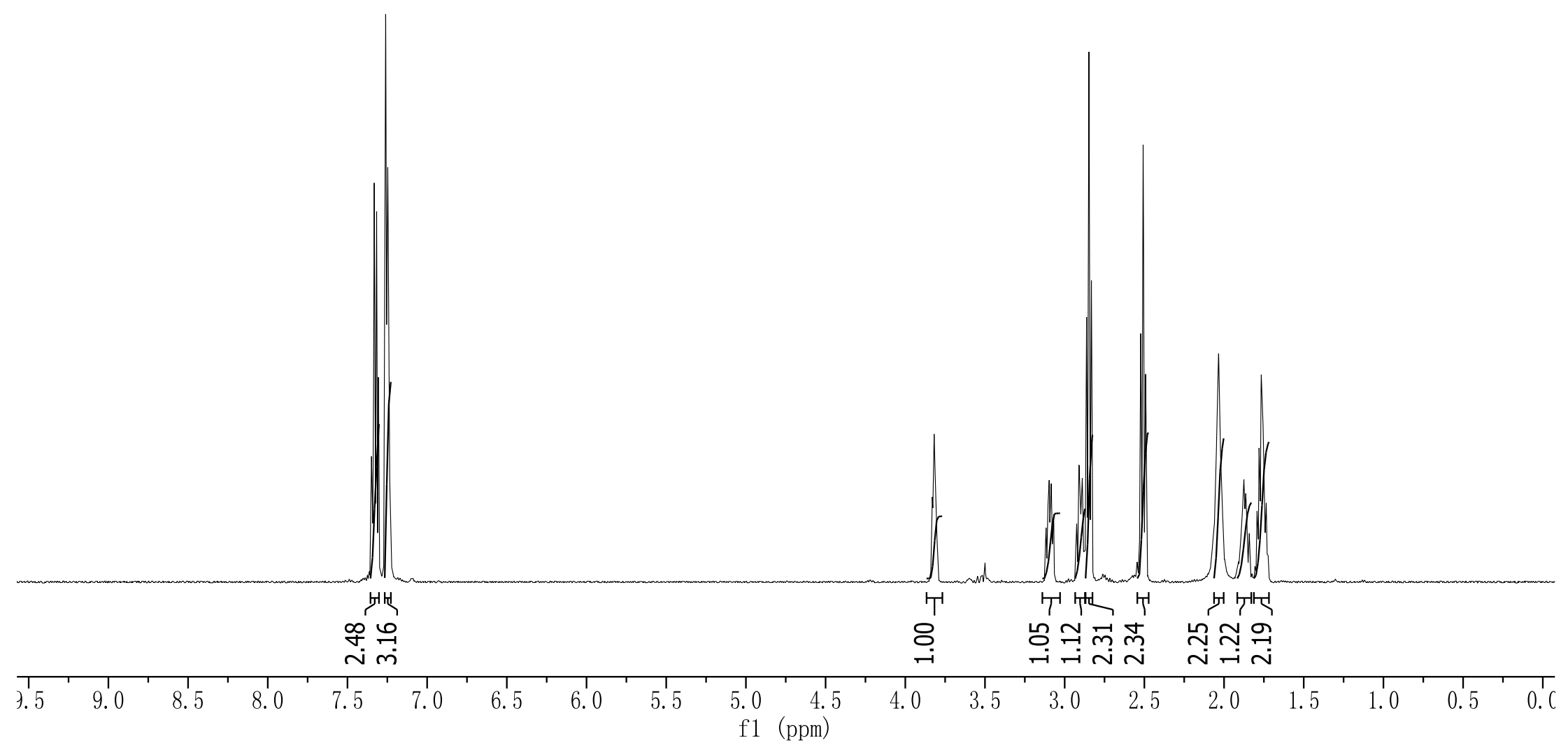




|

${ }^{13} \mathrm{C}-\mathrm{NMR}$ of $( \pm)-5 \mathbf{i}$ in $\mathrm{CDCl}_{3}$
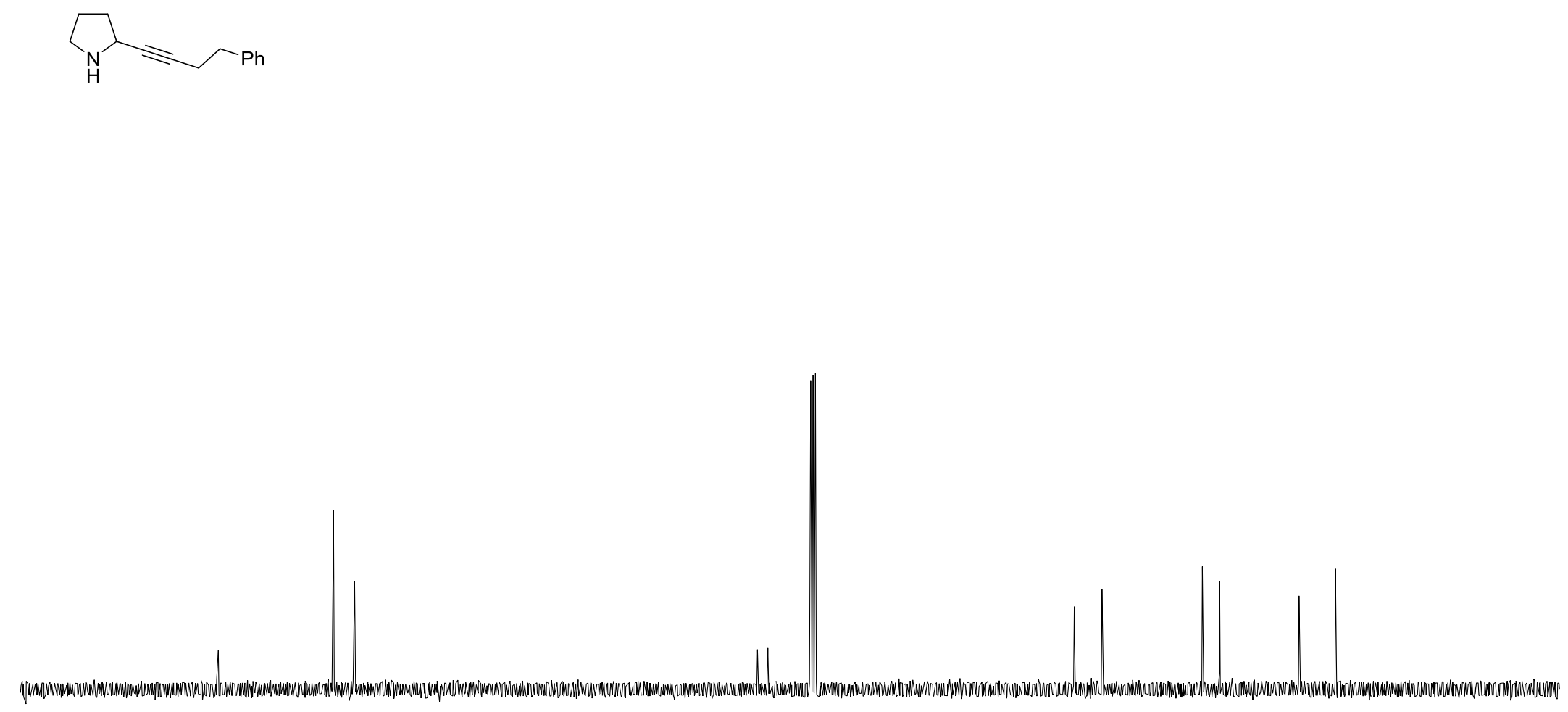

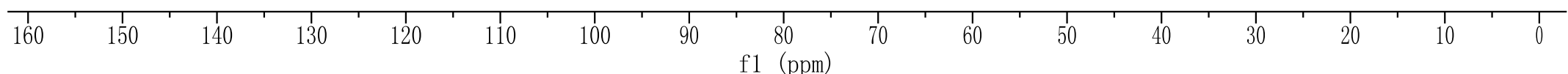




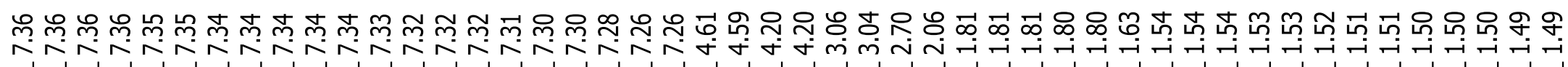

${ }^{1} \mathrm{H}-\mathrm{NMR}$ of $( \pm)-5 \mathbf{j}$ in $\mathrm{CDCl}_{3}$
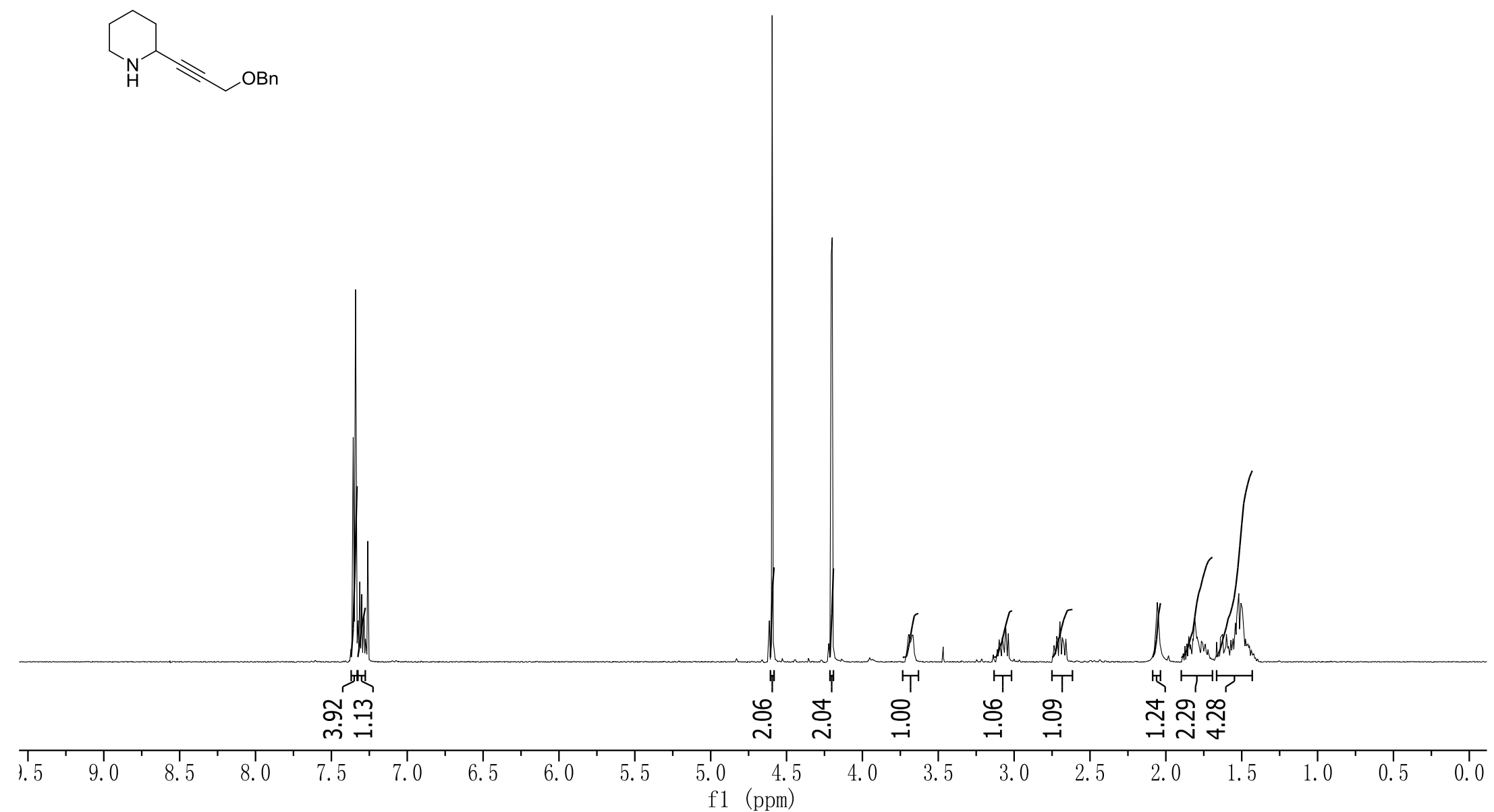


$$
\text { | }
$$

${ }^{13} \mathrm{C}-\mathrm{NMR}$ of $( \pm)-\mathbf{5} \mathbf{j}$ in $\mathrm{CDCl}_{3}$<smiles>C(#CC1CCCCN1)COCc1ccccc1</smiles>

o

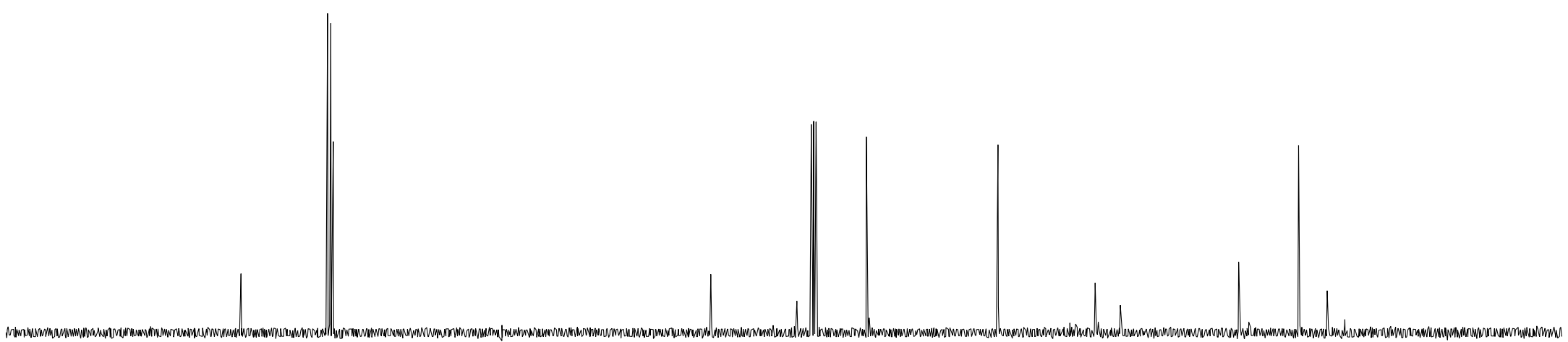

160 150 40 130 120 110 100 


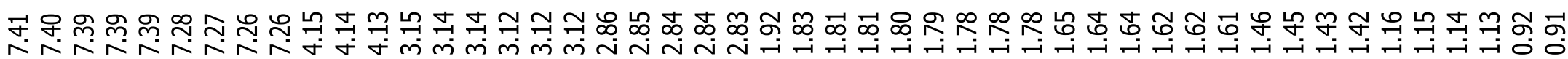
r

${ }^{1} \mathrm{H}$-NMR of $( \pm)-5 \mathbf{k}$ in $\mathrm{CDCl}_{3}$
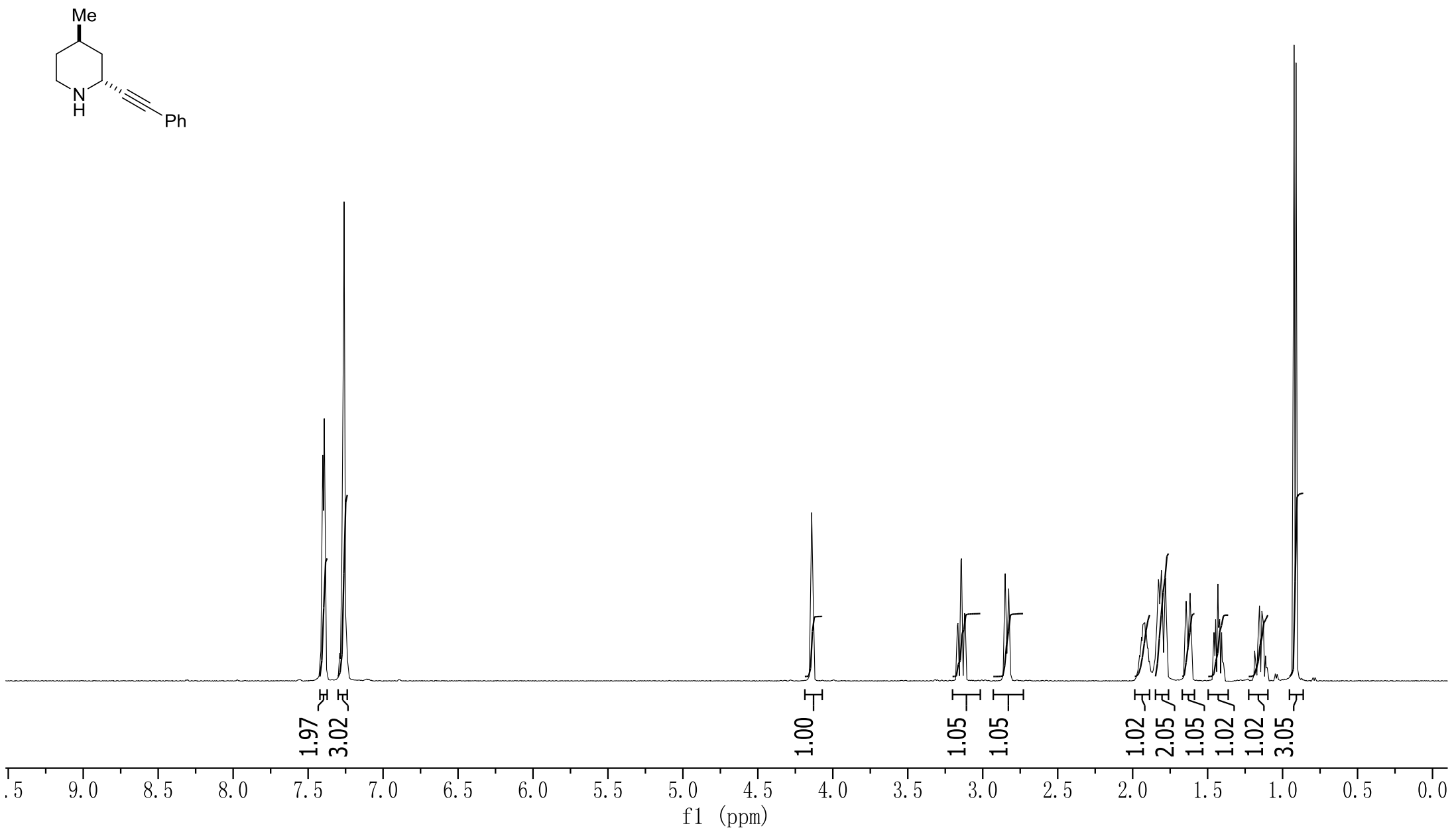
용끄음

ตำ

$1 \mid 1$

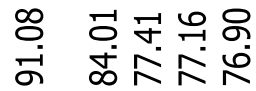

का

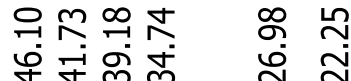

$$
\begin{aligned}
& 1111 \text { | }
\end{aligned}
$$

${ }^{13} \mathrm{C}-\mathrm{NMR}$ of $( \pm)-\mathbf{5 k}$ in $\mathrm{CDCl}_{3}$

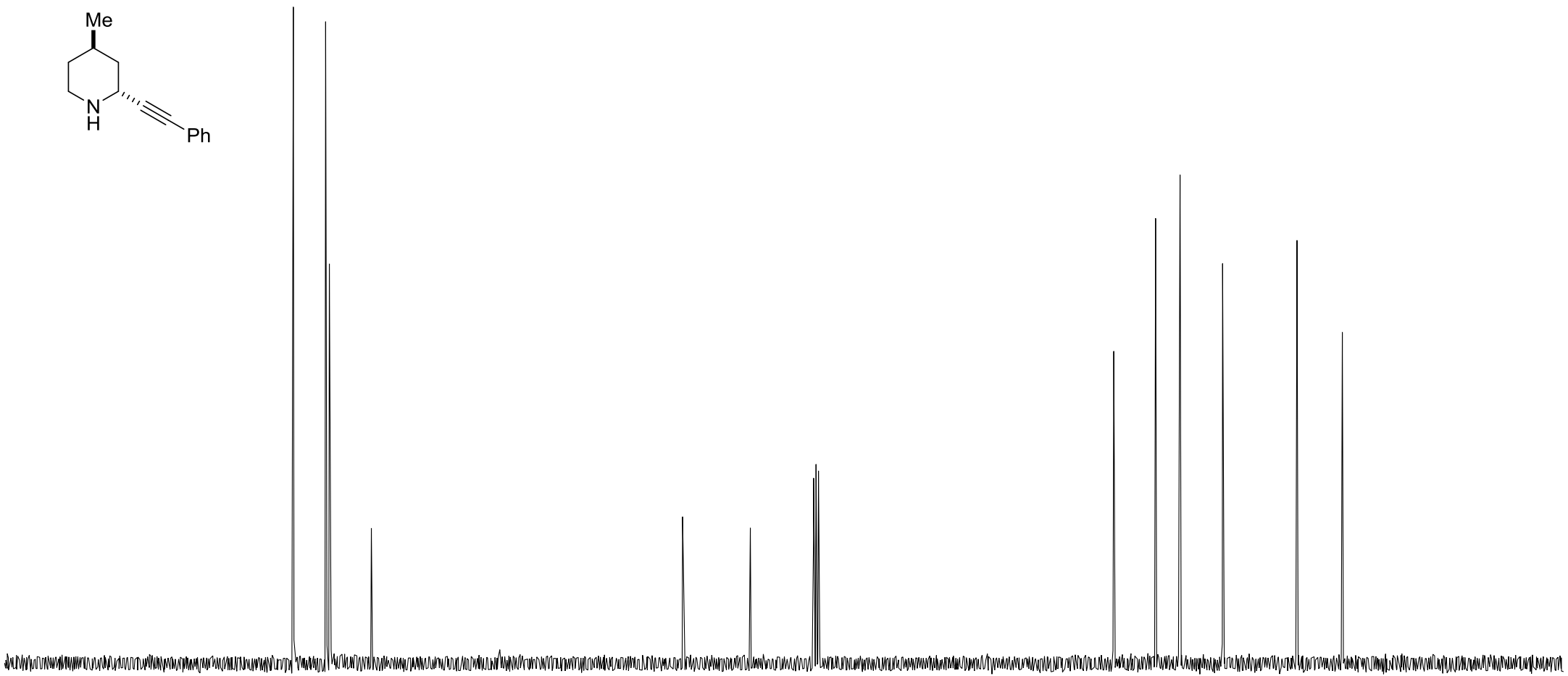

$\frac{1}{160}$

150

140

130
130

10

100

90

f1 (ppm) 


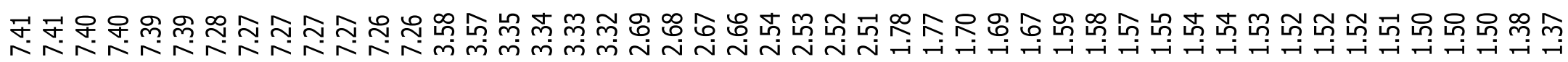

${ }^{1} \mathrm{H}-\mathrm{NMR}$ of $( \pm)-5 \mathrm{I}$ in $\mathrm{CDCl}_{3}$

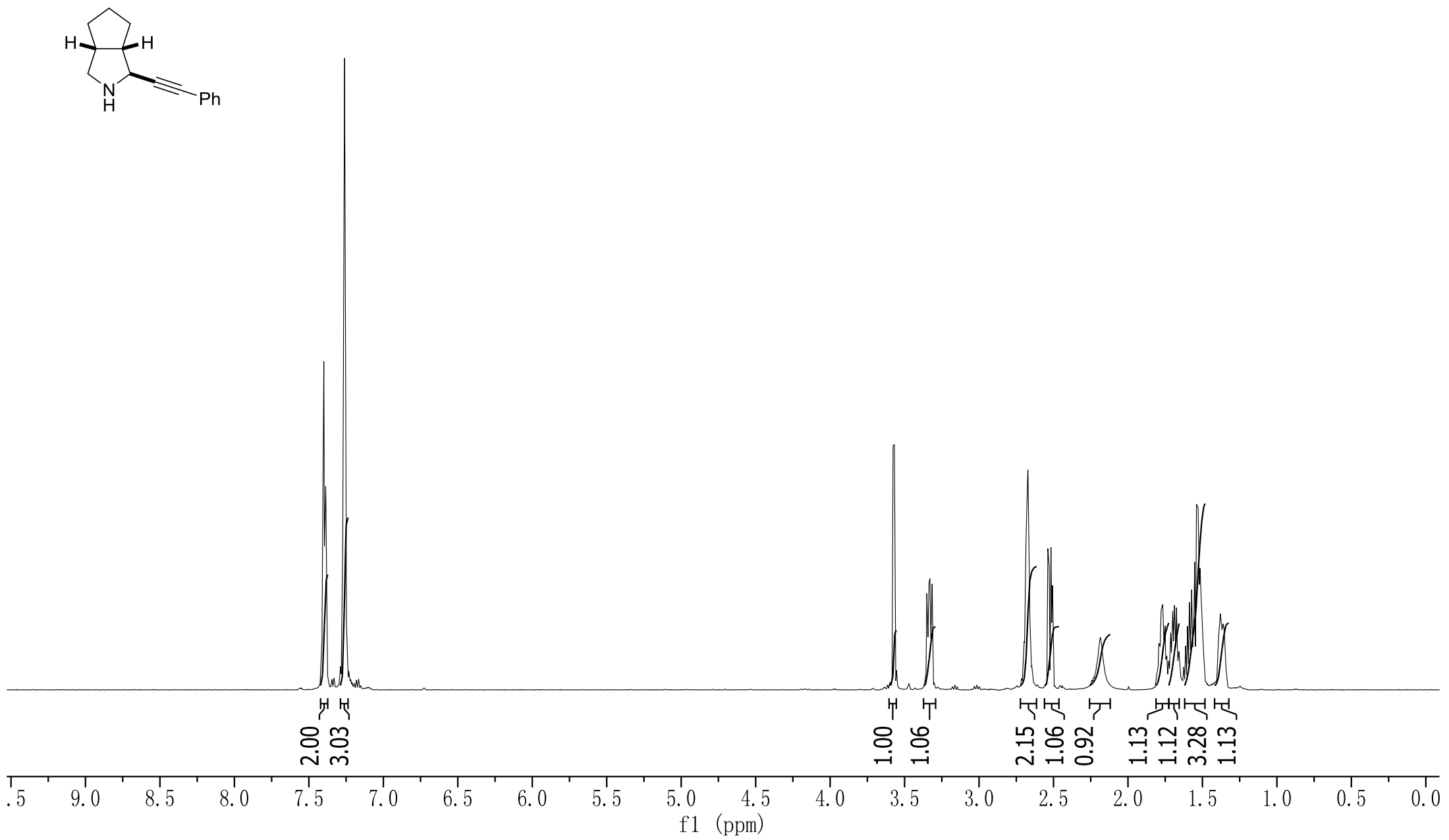


요마

ตํํำ

$|\backslash|$

${ }^{13} \mathrm{C}-\mathrm{NMR}$ of $( \pm)-5 \mathrm{I}$ in $\mathrm{CDCl}_{3}$

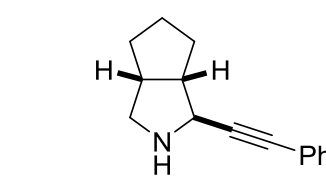

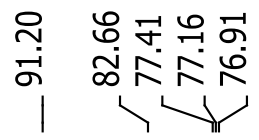

గ్ర

एं

111
ธํㅛㅇㅠ.

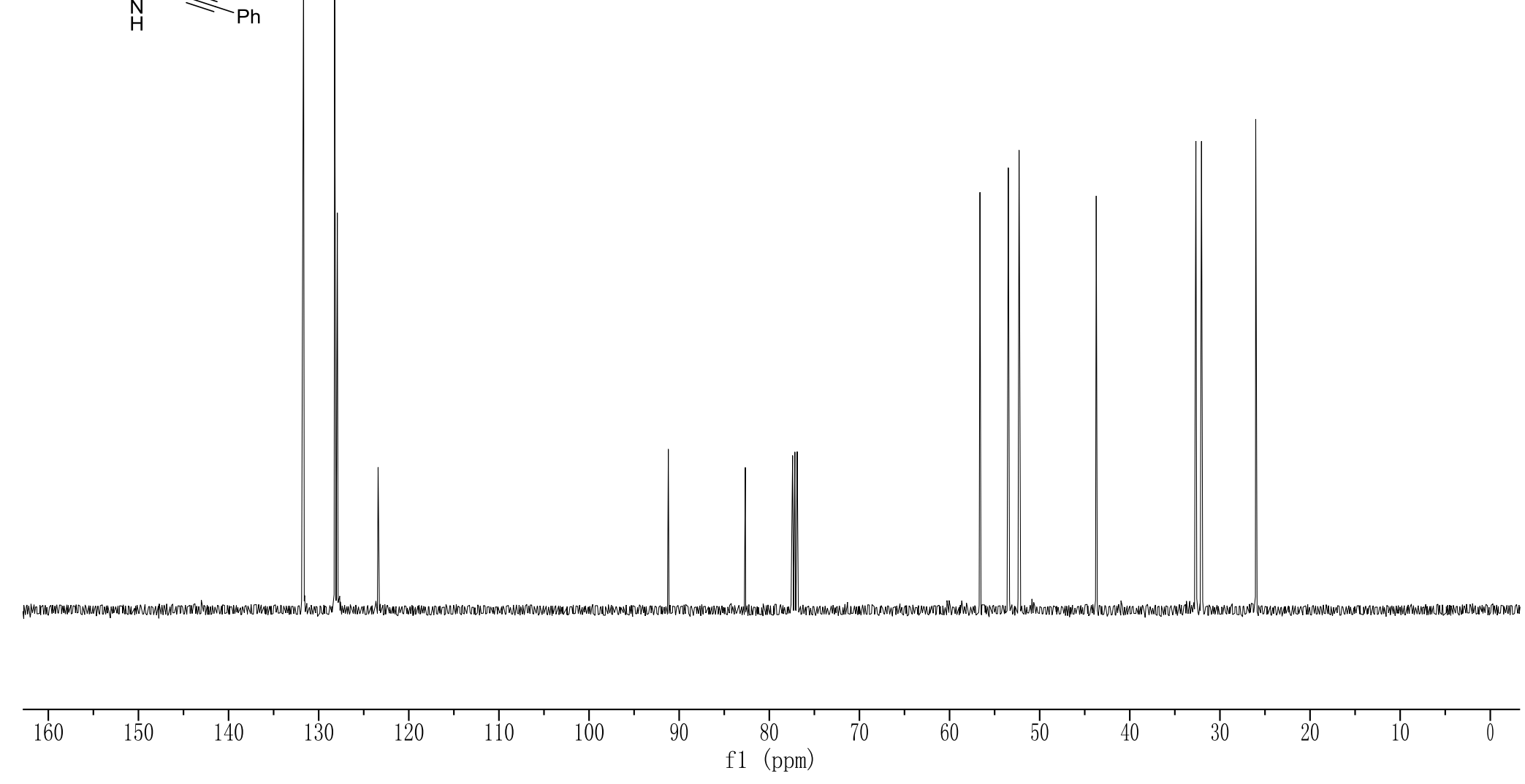




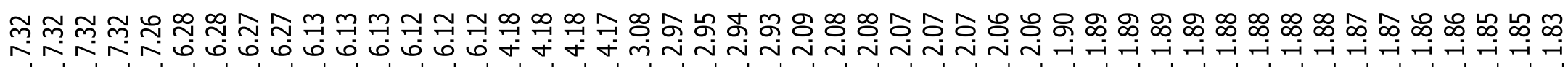

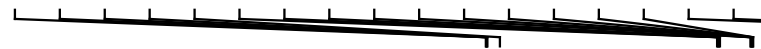

${ }^{1} \mathrm{H}-\mathrm{NMR}$ of $( \pm)-6 \mathbf{a}$ in $\mathrm{CDCl}_{3}$<smiles>c1coc(C2CCCN2)c1</smiles>

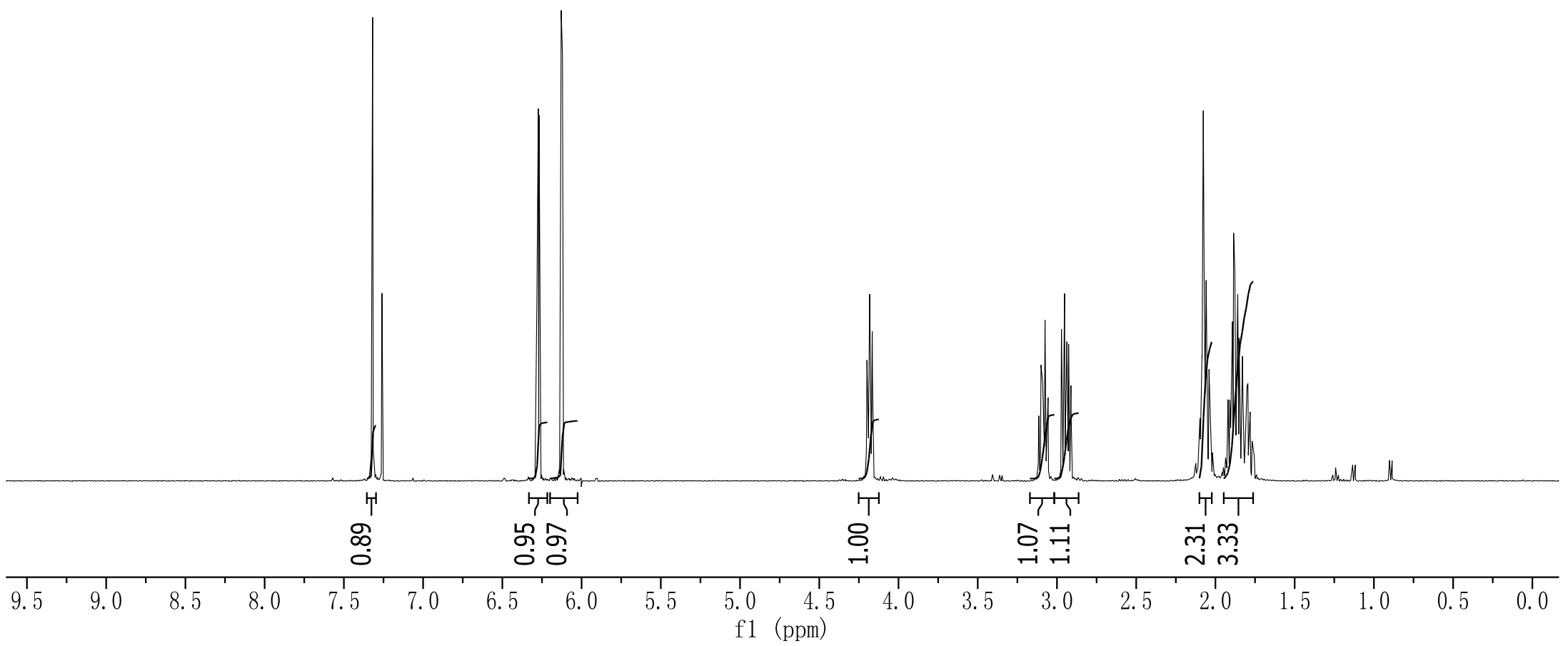




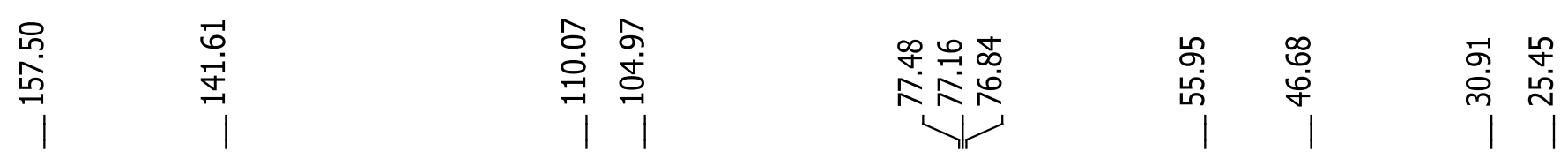

${ }^{13} \mathrm{C}-\mathrm{NMR}$ of $( \pm)$ - $\mathbf{6} \mathbf{a}$ in $\mathrm{CDCl}_{3}$

$\langle\mathrm{H}$

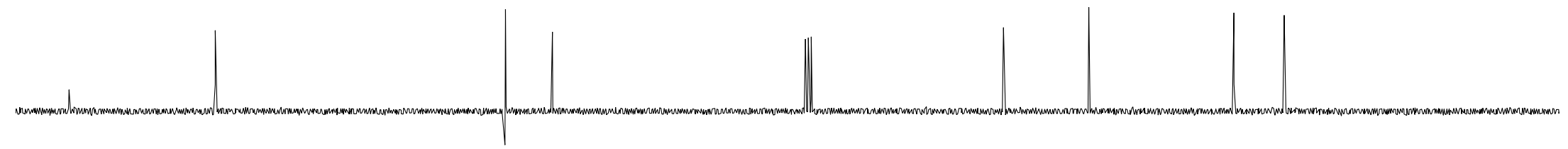

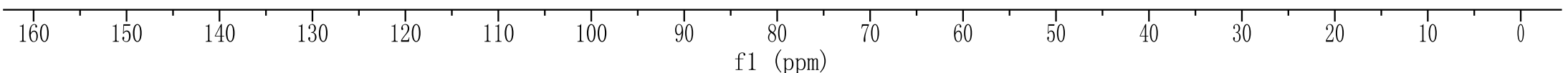




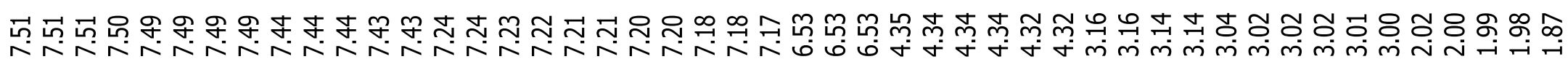

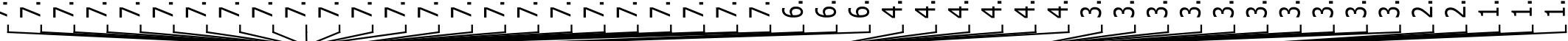

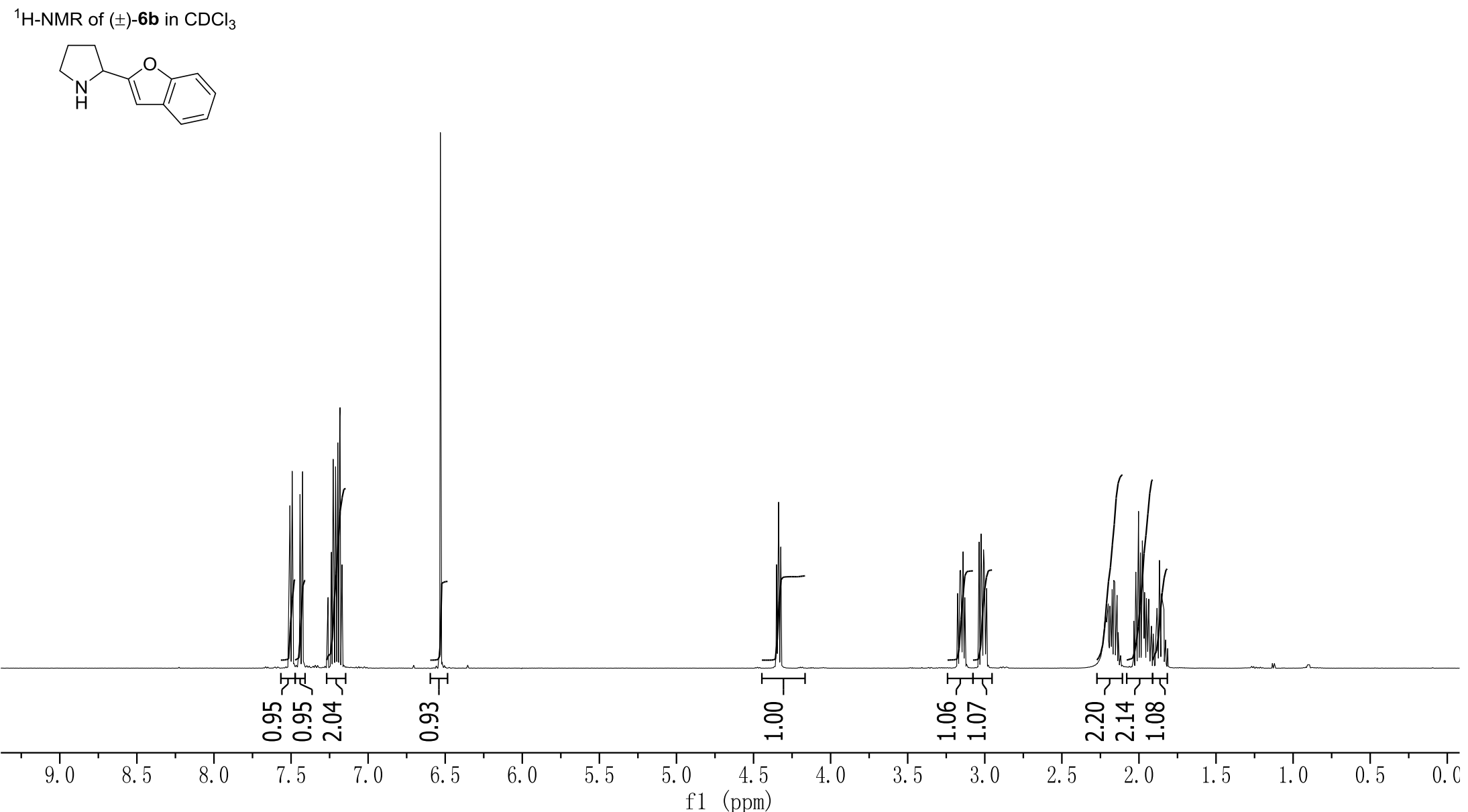




\begin{tabular}{|c|c|c|c|c|c|c|c|}
\hline 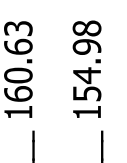 & 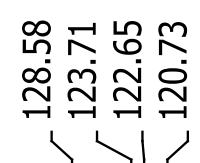 & $\stackrel{亏}{\stackrel{F}{G}}$ & గ్ & 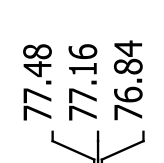 & $\begin{array}{l}\text { ปี } \\
\text { ம் }\end{array}$ & $\begin{array}{l}\hat{\infty} \\
\dot{f} \\
\dot{f}\end{array}$ & 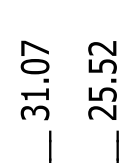 \\
\hline
\end{tabular}

${ }^{13} \mathrm{C}-\mathrm{NMR}$ of $( \pm)-6 \mathbf{b}$ in $\mathrm{CDCl}_{3}$<smiles>c1ccc2oc(C3CCCN3)cc2c1</smiles>

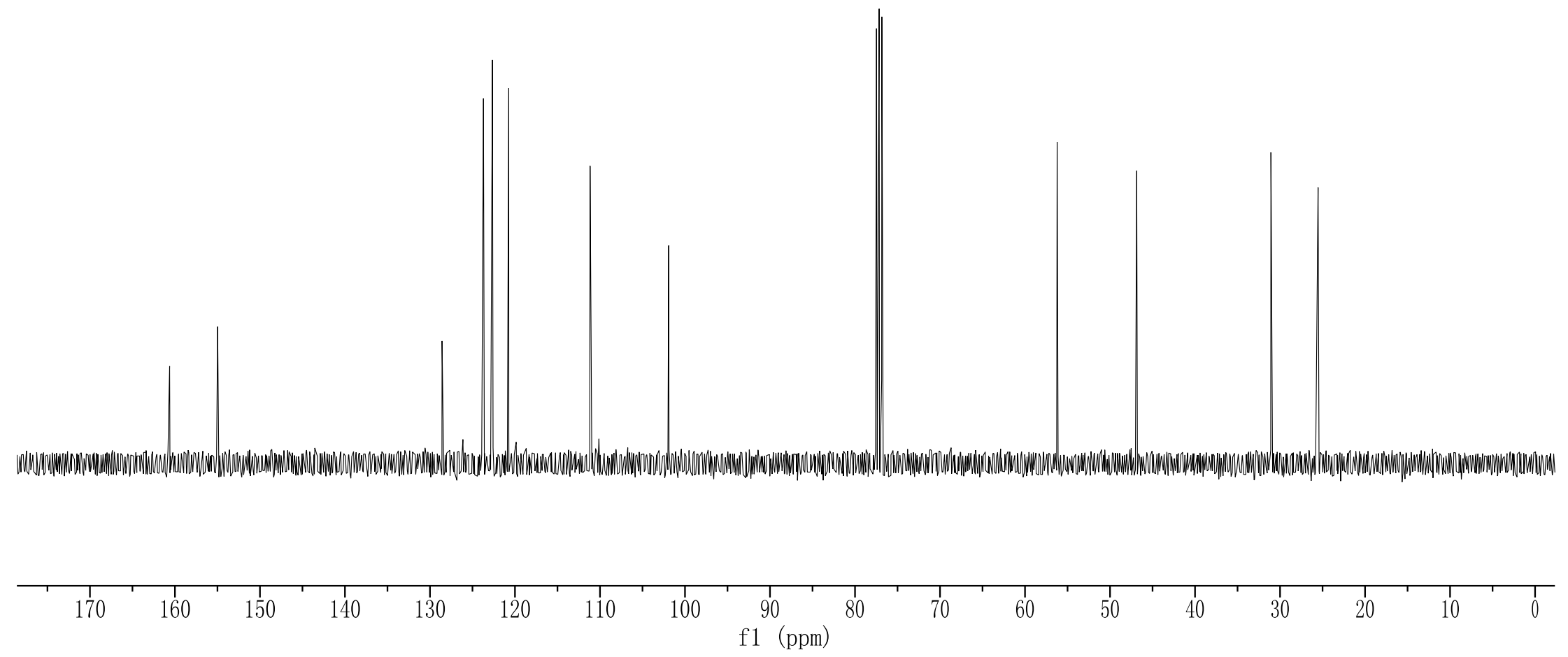




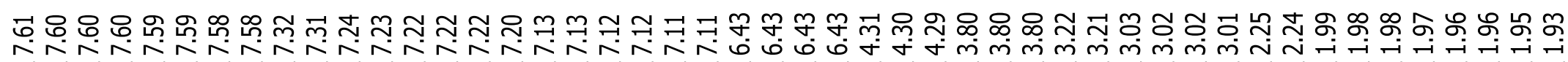

${ }^{1} \mathrm{H}-\mathrm{NMR}$ of $( \pm)-6 \mathrm{c}$ in $\mathrm{CDCl}_{3}$
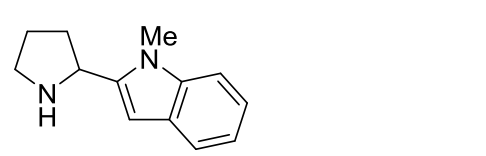


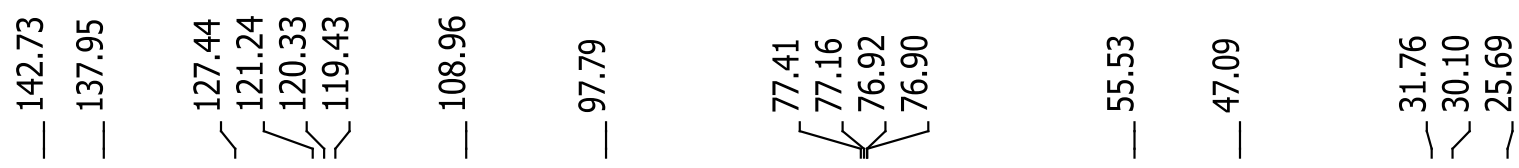

${ }^{13} \mathrm{C}-\mathrm{NMR}$ of $( \pm)-6 \mathrm{c}$ in $\mathrm{CDCl}_{3}$
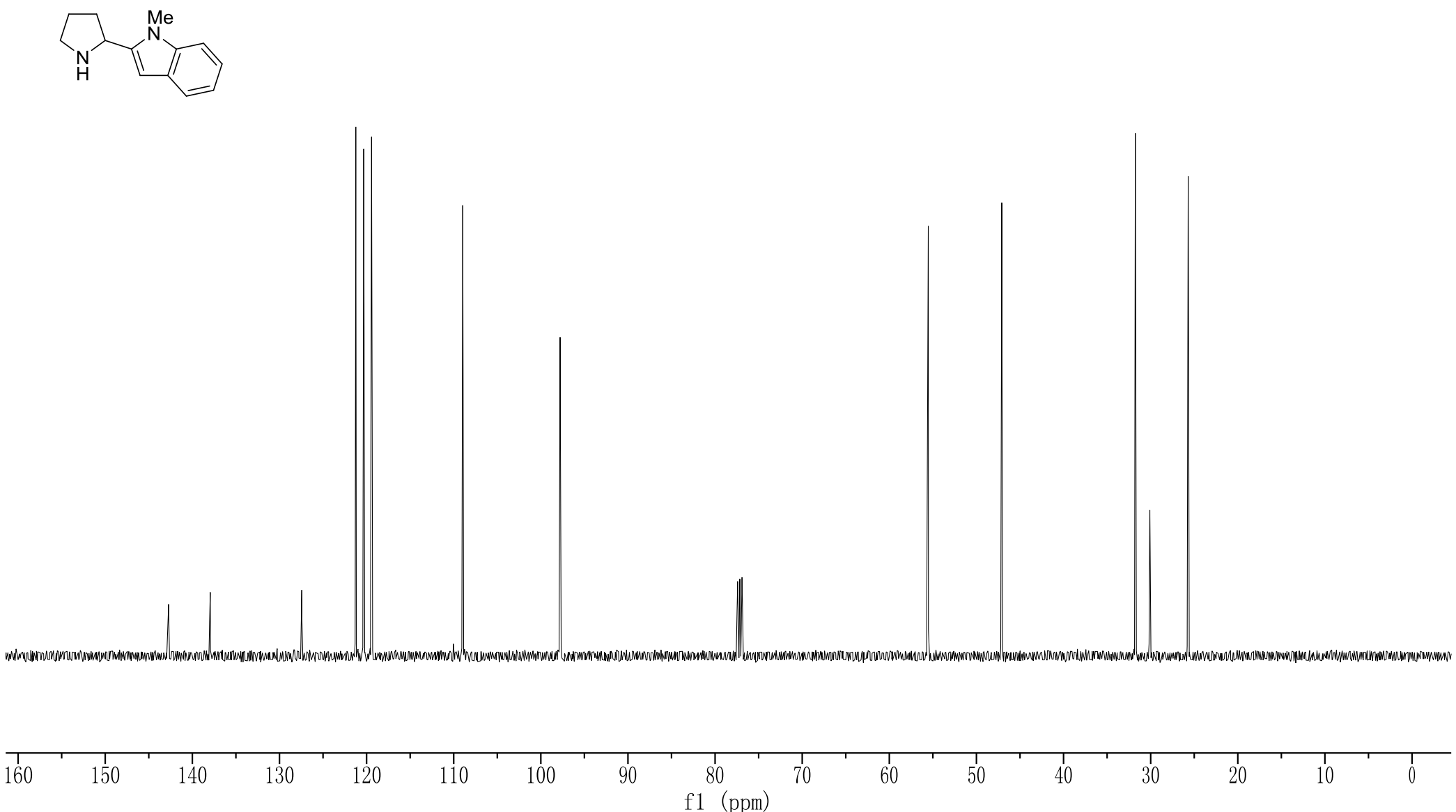


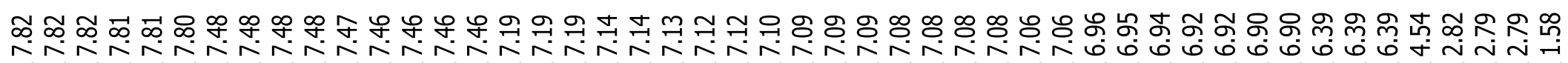
$\underset{L}{2+L}+$

${ }^{1} \mathrm{H}-\mathrm{NMR}$ of $( \pm)-6 \mathbf{d}$ in $\mathrm{CDCl}_{3}$
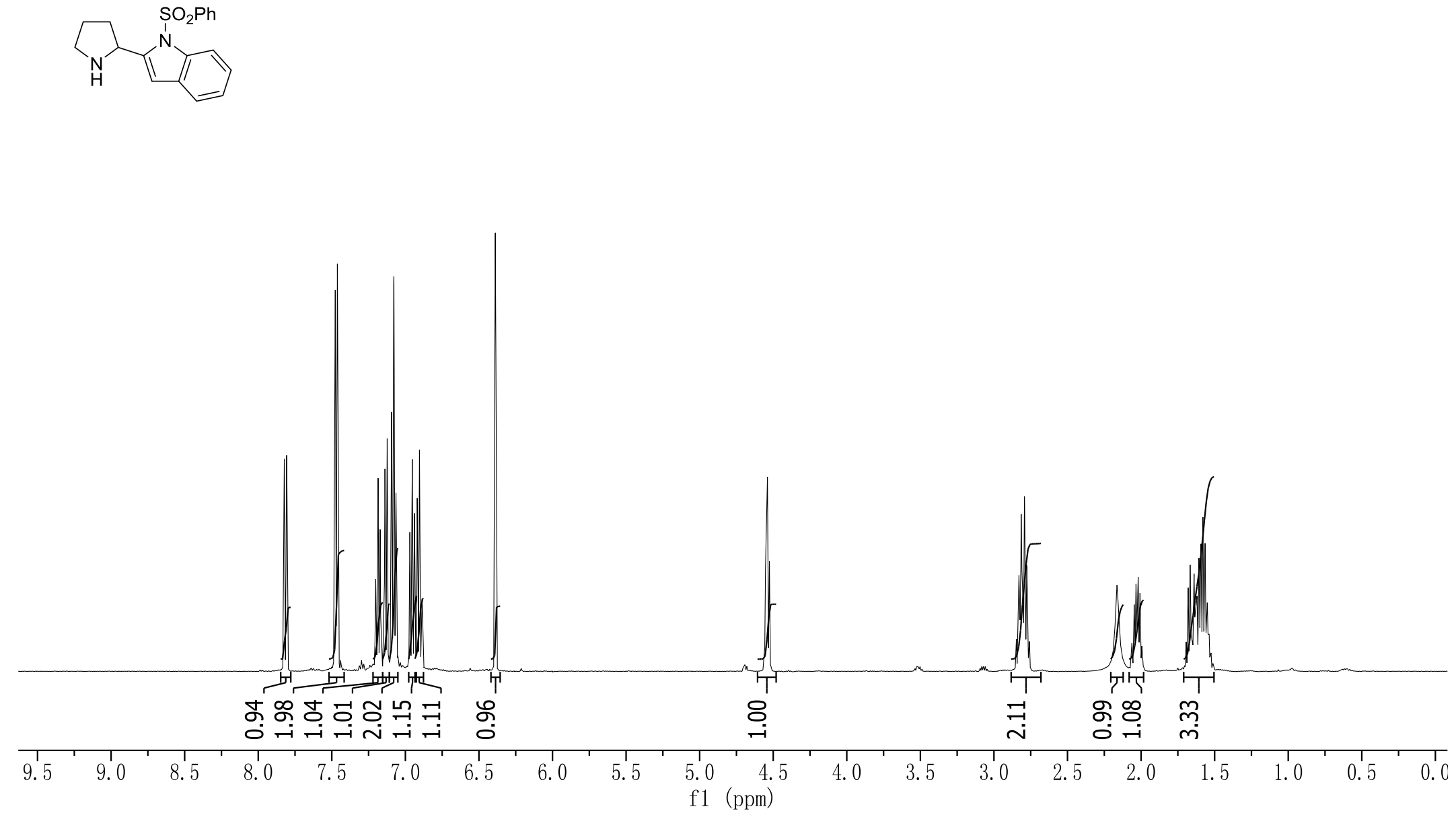


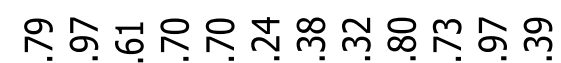

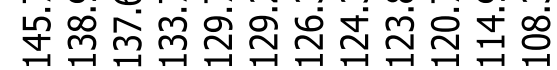

। रो। ।
₹드

ลำ

4

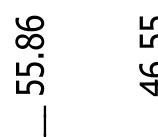

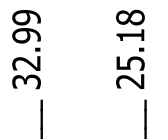

${ }^{13} \mathrm{C}-\mathrm{NMR}$ of $( \pm)-\mathbf{6 d}$ in $\mathrm{CDCl}_{3}$

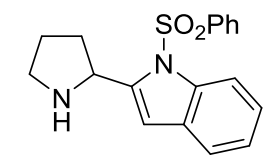

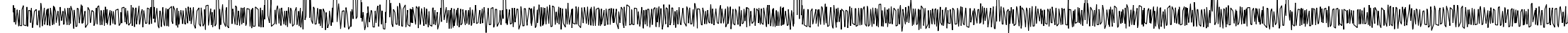

$\stackrel{1}{60}$

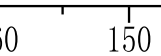

150

40

130

120

110

100

90

1
$70 \quad 60$

50 
거 겅 궁

${ }^{1} \mathrm{H}-\mathrm{NMR}$ of $( \pm)-6 \mathbf{e}$ in $\mathrm{CDCl}_{3}$
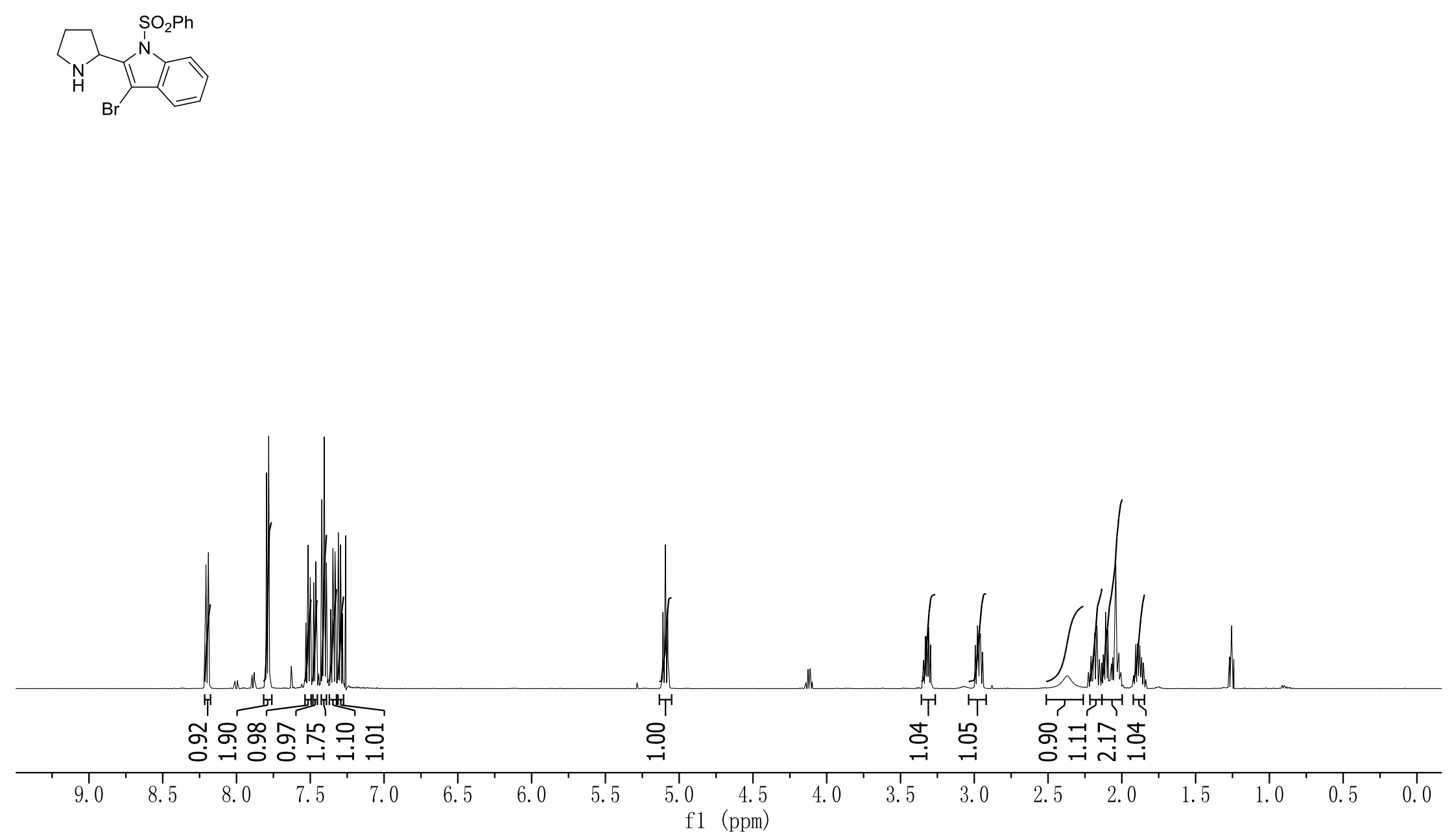


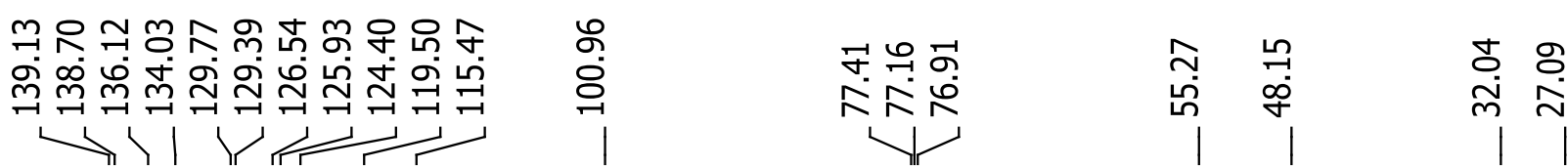

${ }^{13} \mathrm{C}-\mathrm{NMR}$ of $( \pm)-6 \mathrm{e}$ in $\mathrm{CDCl}_{3}$
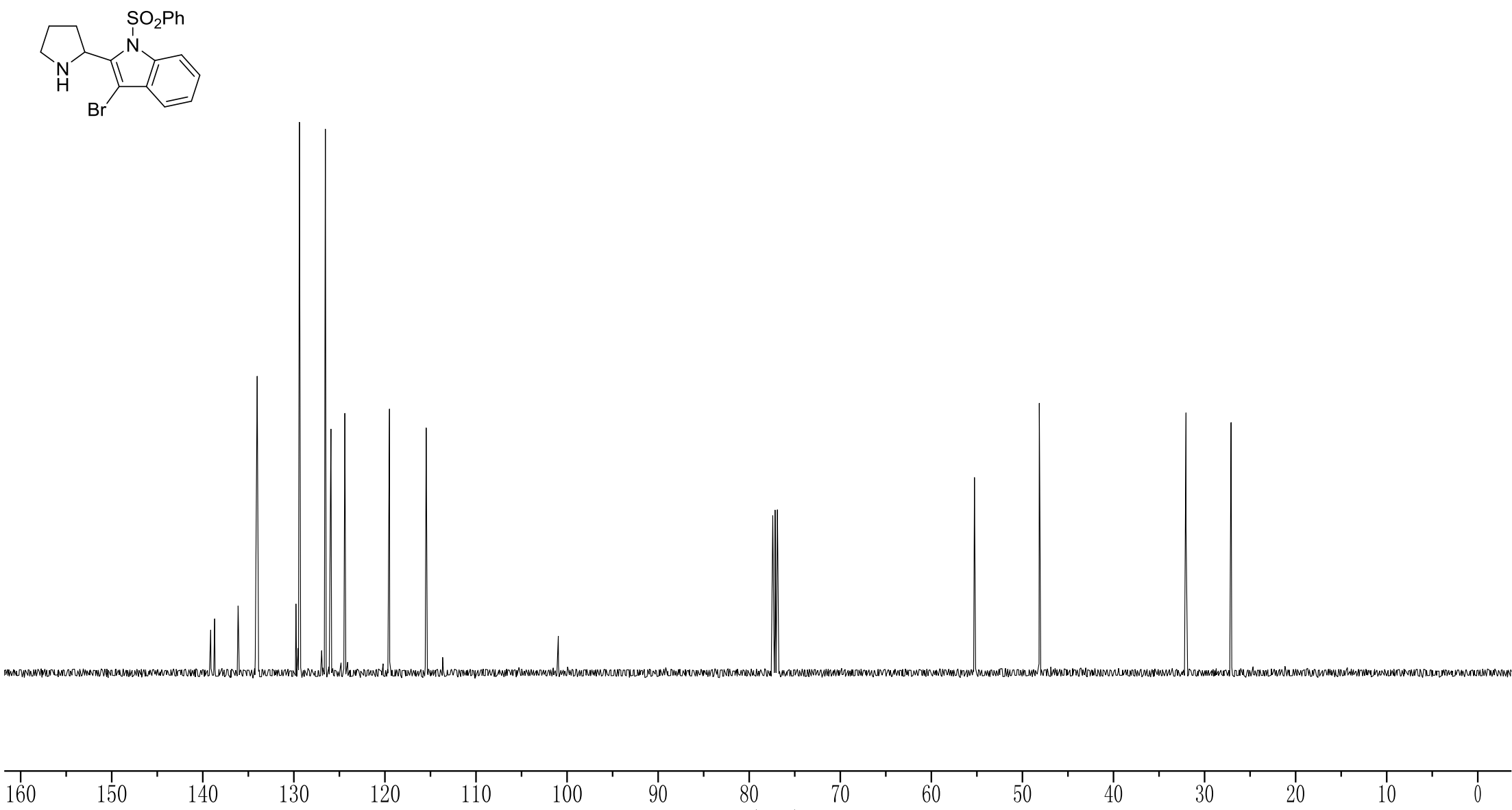

f1 (ppm) 


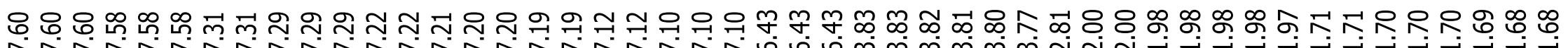
L

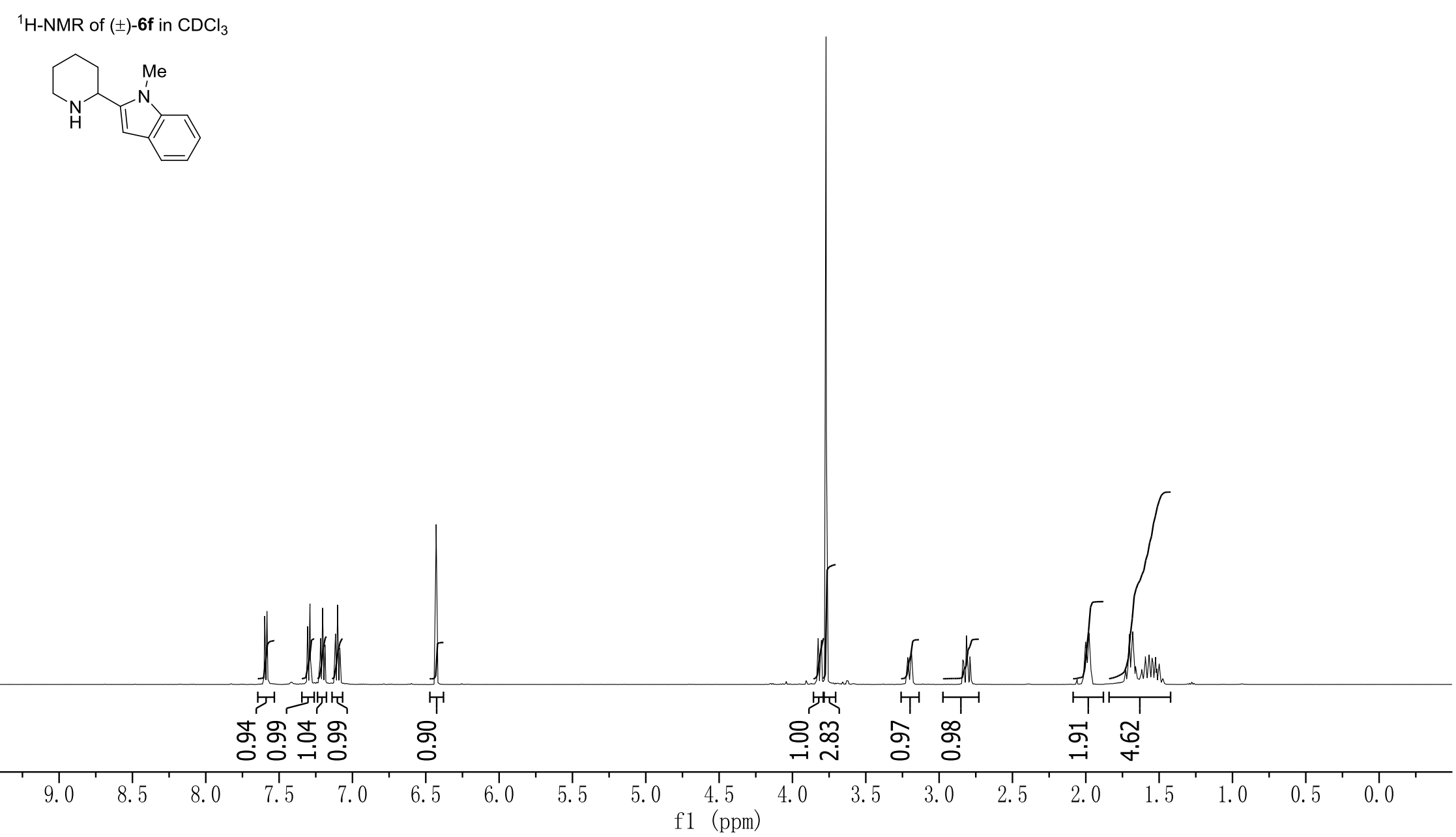




\begin{tabular}{|c|c|c|c|c|c|c|c|}
\hline 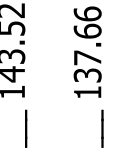 & 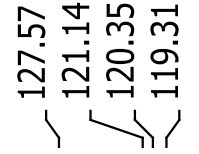 & $\begin{array}{l}\text { o. } \\
\text { o } \\
0 \\
0 \\
1\end{array}$ & $\begin{array}{l}\hat{O} \\
\infty \\
o\end{array}$ & 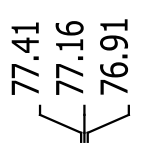 & 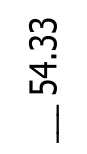 & $\stackrel{\overrightarrow{\tilde{r}}}{\stackrel{f}{f}}$ & 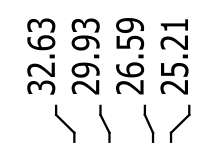 \\
\hline
\end{tabular}

${ }^{13} \mathrm{C}-\mathrm{NMR}$ of $( \pm)-6 \mathrm{f}$ in $\mathrm{CDCl}_{3}$
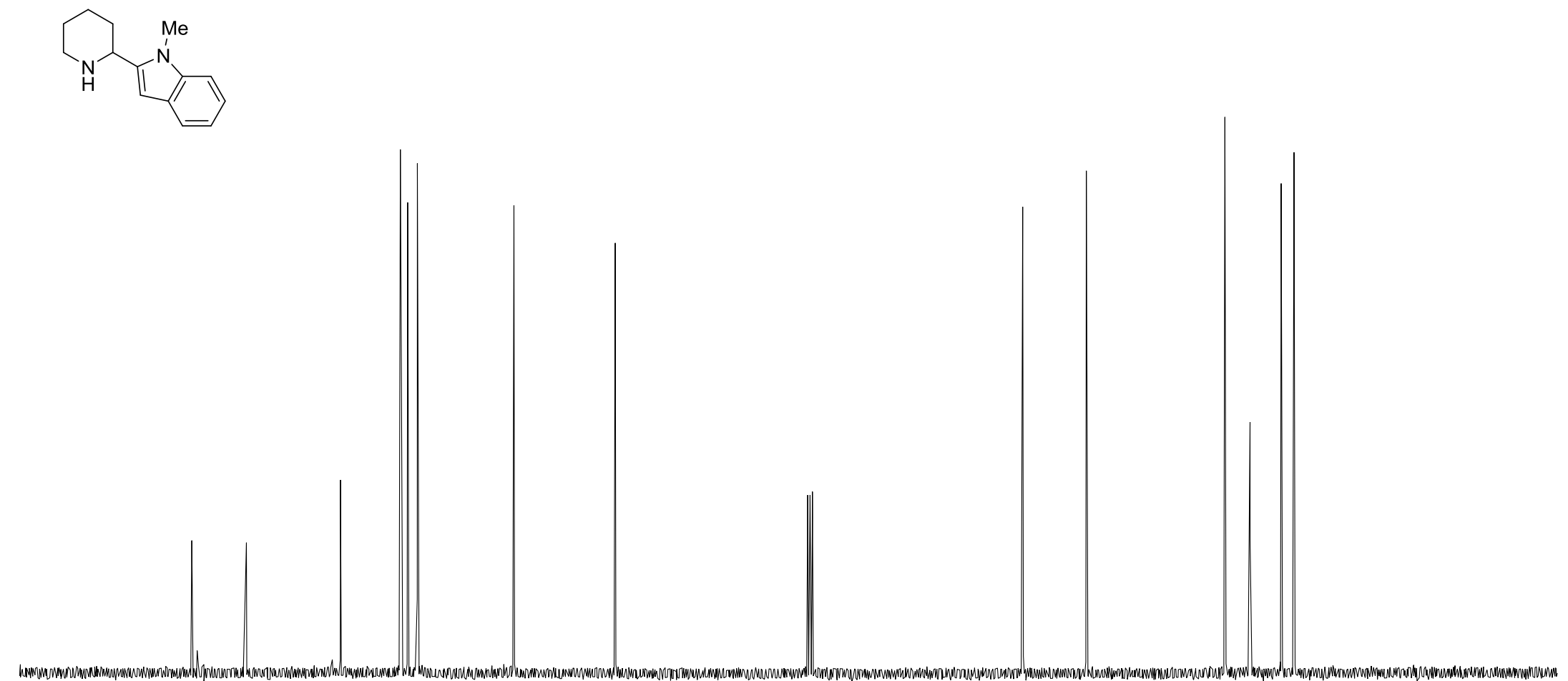

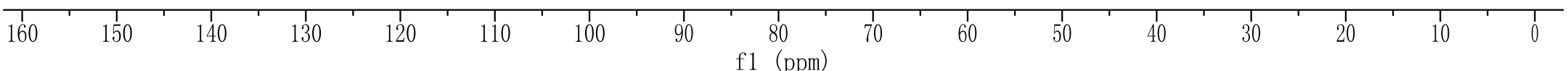




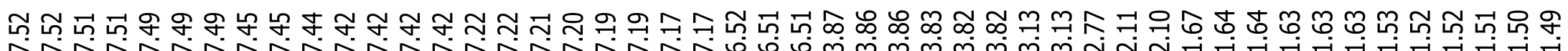
供

${ }^{1} \mathrm{H}-\mathrm{NMR}$ of $( \pm)-6 \mathrm{~g}$ in $\mathrm{CDCl}_{3}$
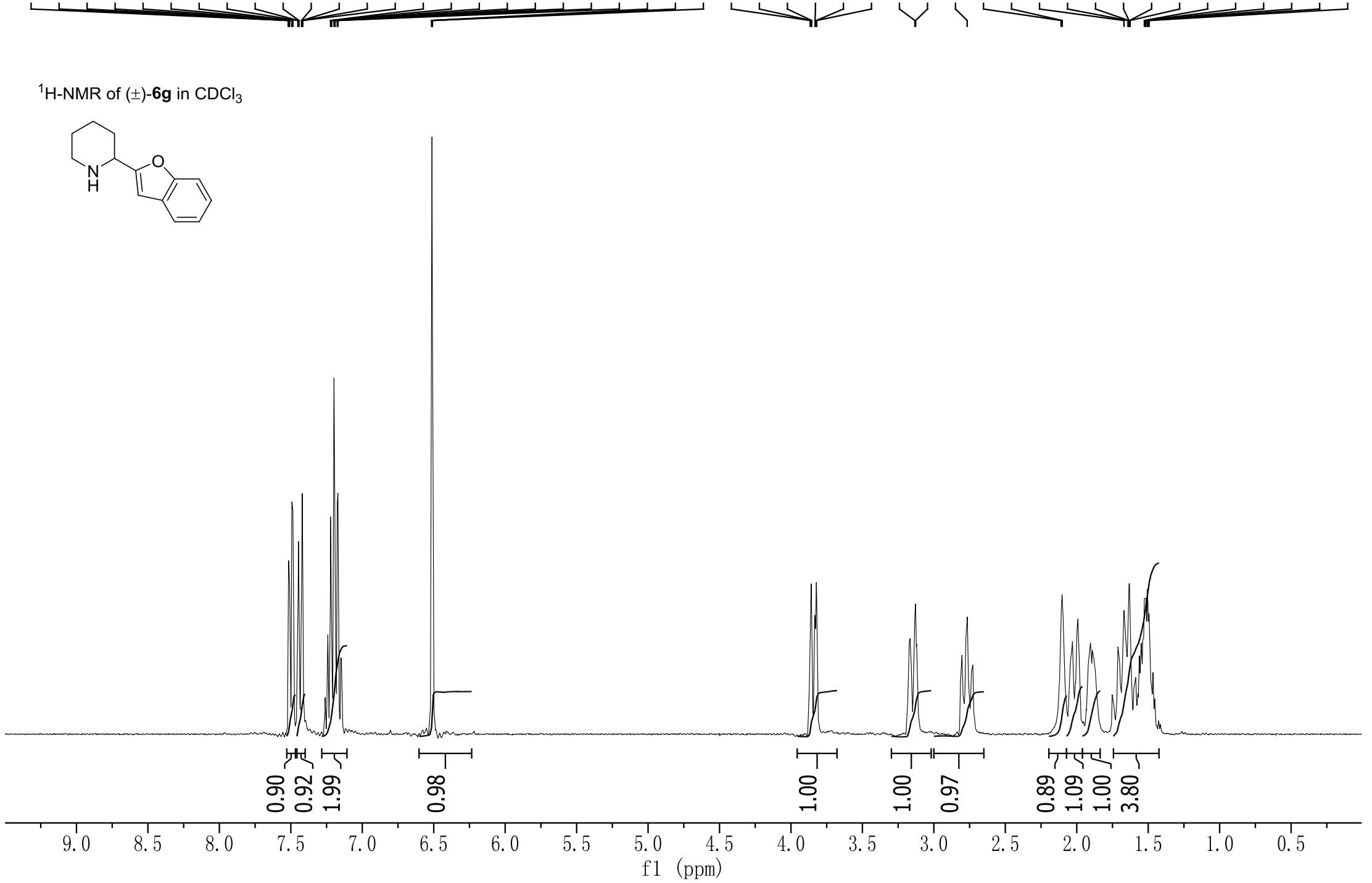


\begin{tabular}{|c|c|c|c|c|c|c|}
\hline 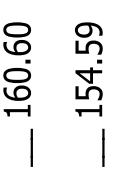 & 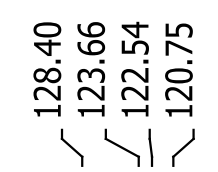 & 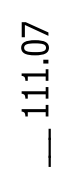 & $\begin{array}{l}\vec{m} \\
\overrightarrow{0} \\
\overrightarrow{0}\end{array}$ & 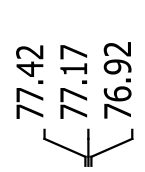 & $\begin{array}{l}8 \\
\text { in } \\
\text { ผn } \\
\text { | }\end{array}$ & $\begin{array}{c}\hat{\infty} \\
\dot{\phi} \\
\end{array}$ \\
\hline
\end{tabular}

${ }^{13} \mathrm{C}-\mathrm{NMR}$ of $( \pm)-6 \mathrm{~g}$ in $\mathrm{CDCl}_{3}$

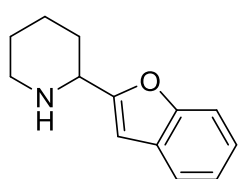

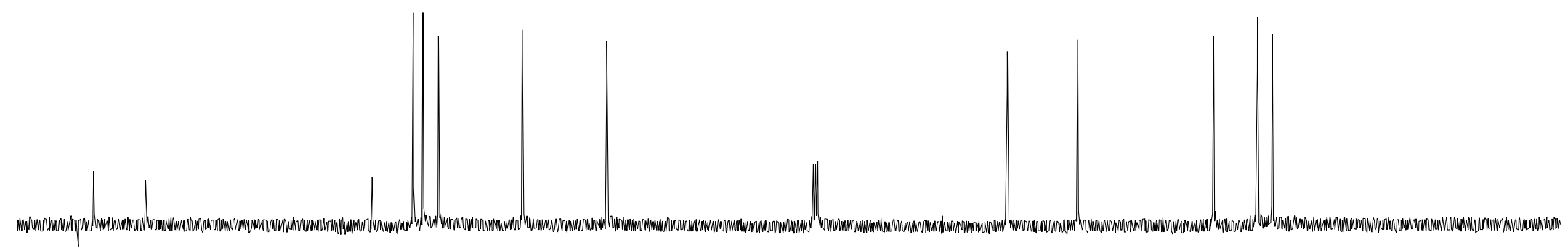

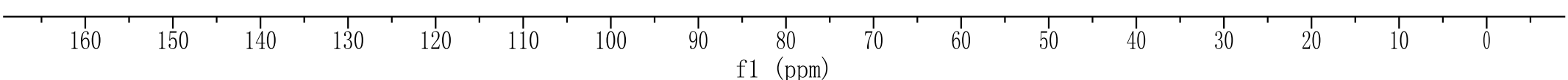




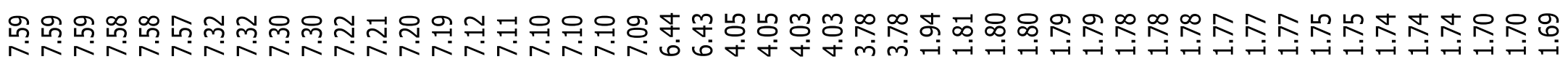

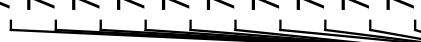

${ }^{1} \mathrm{H}-\mathrm{NMR}$ of $( \pm)-6 \mathbf{h}$ in $\mathrm{CDCl}_{3}$

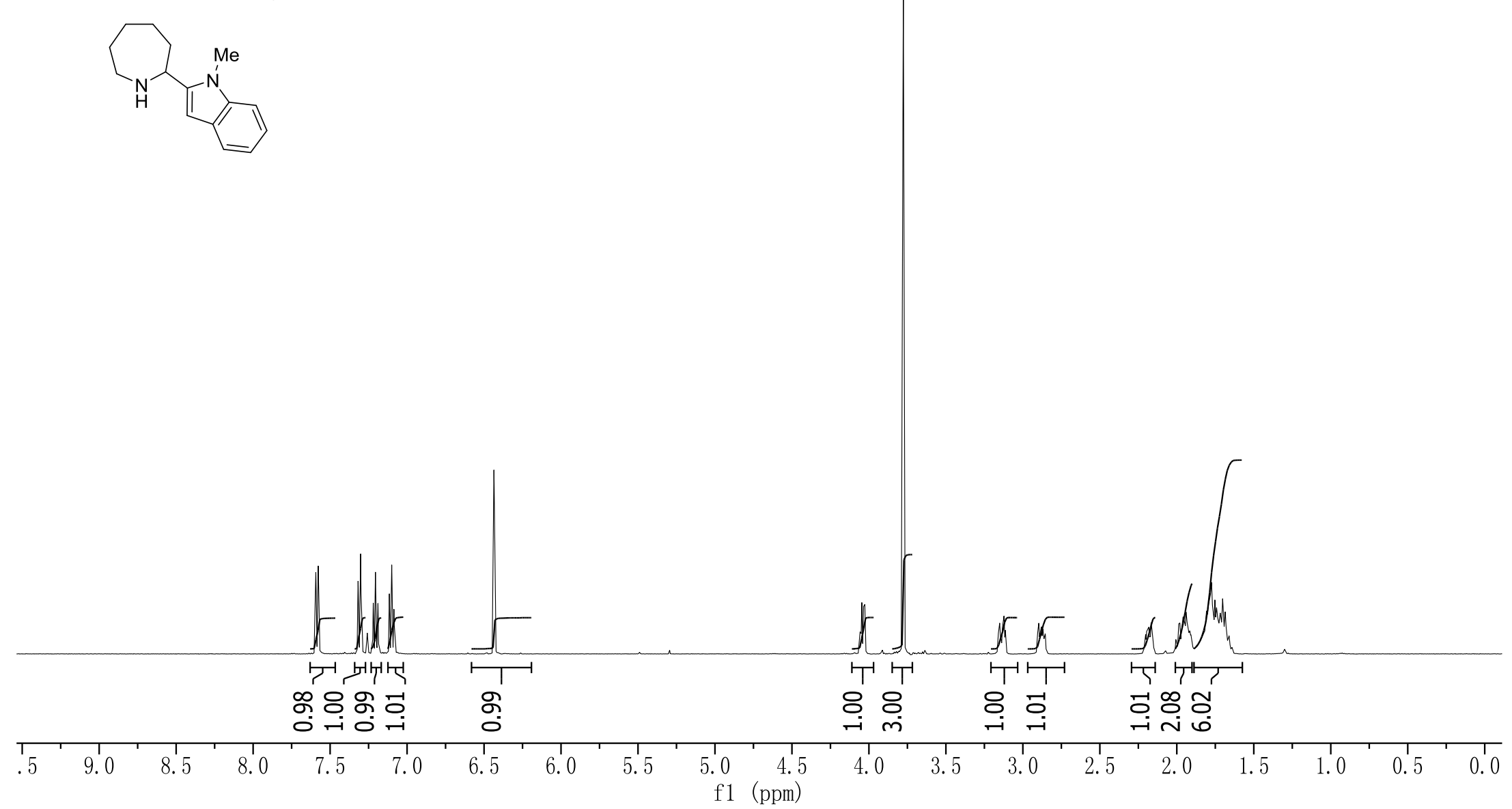




\begin{tabular}{|c|c|c|c|c|c|c|c|}
\hline 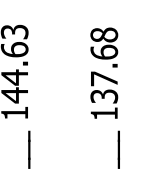 & 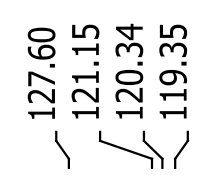 & $\begin{array}{l}\text { o } \\
0 \\
0 \\
\stackrel{0}{1}\end{array}$ & $\begin{array}{l}\text { g. } \\
\vdots \\
\vdots\end{array}$ & | & 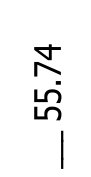 & 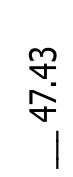 & 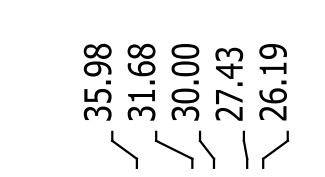 \\
\hline
\end{tabular}

${ }^{13} \mathrm{C}-\mathrm{NMR}$ of $( \pm)-6 \mathrm{~h}$ in $\mathrm{CDCl}_{3}$
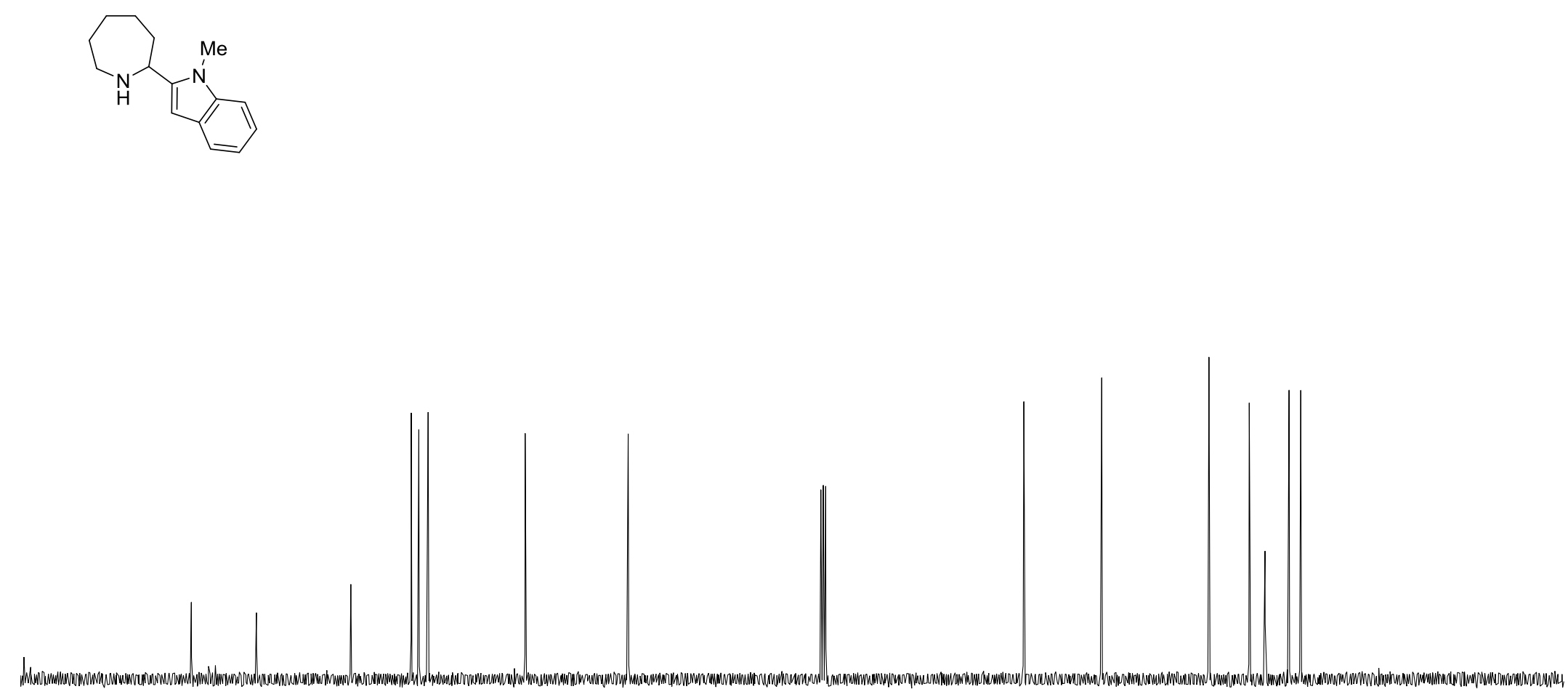

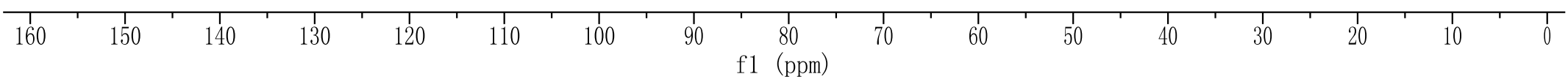




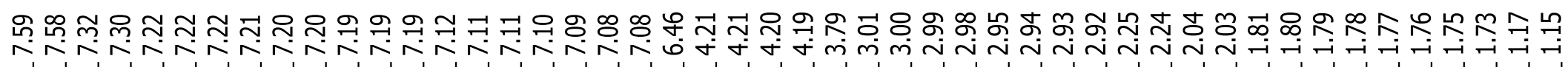

r

${ }^{1} \mathrm{H}-\mathrm{NMR}$ of $( \pm)-6 \mathbf{i}$ in $\mathrm{CDCl}_{3}$
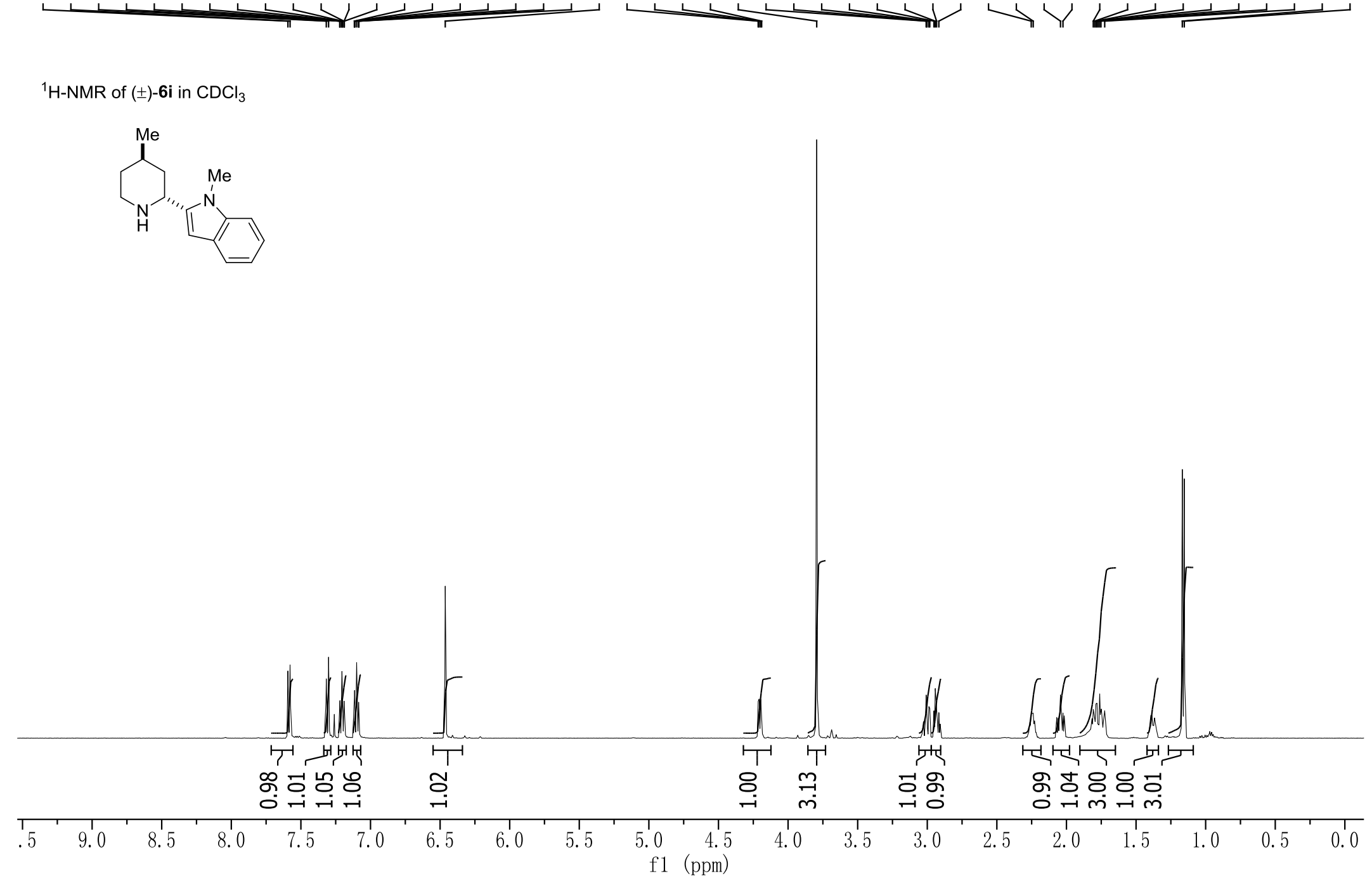


\begin{tabular}{|c|c|c|c|c|c|}
\hline 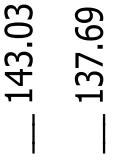 & 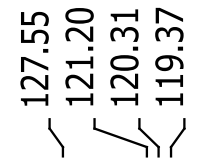 & $\begin{array}{l}\text { ô } \\
\infty \\
0 \\
0 \\
\mid\end{array}$ & $\begin{array}{l}\text { on } \\
\infty \\
\infty\end{array}$ & 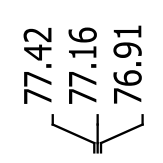 & 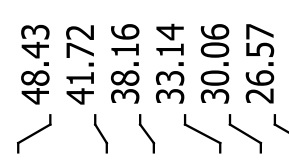 \\
\hline
\end{tabular}

${ }^{13} \mathrm{C}-\mathrm{NMR}$ of $( \pm)-6 \mathrm{i}$ in $\mathrm{CDCl}_{3}$
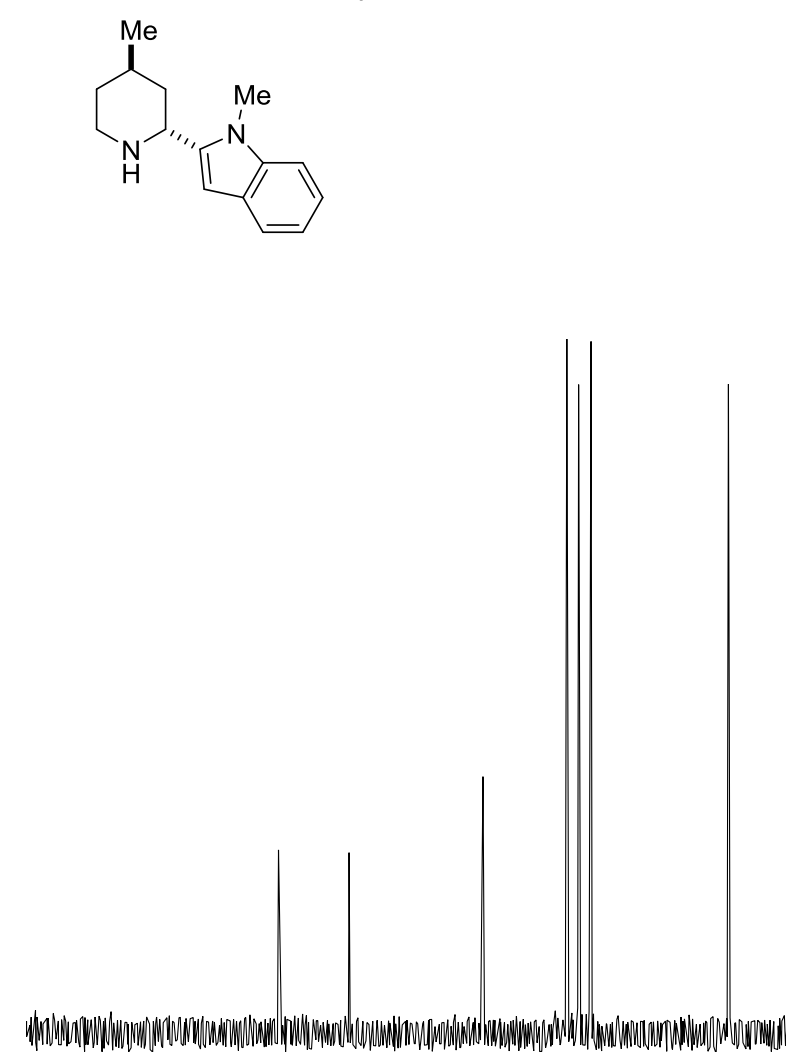

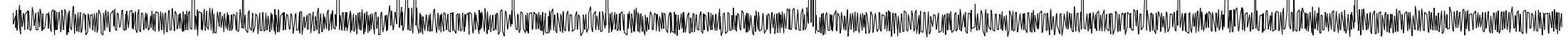

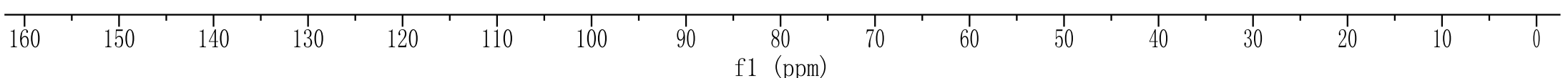




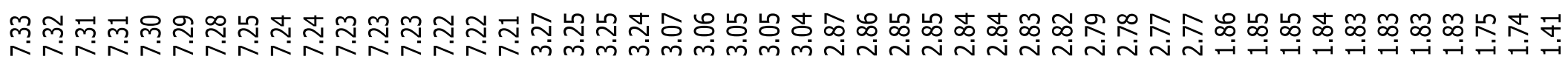

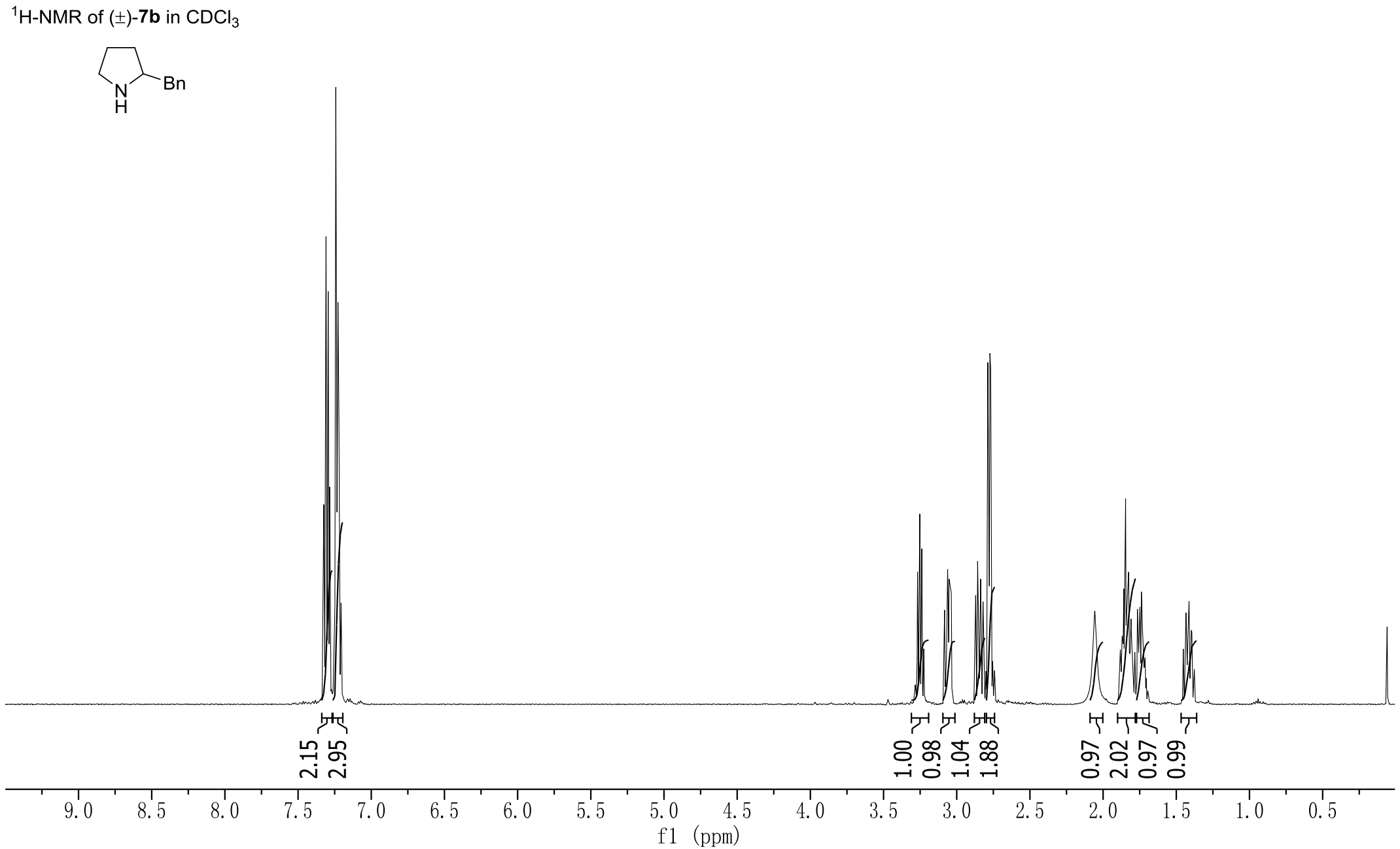




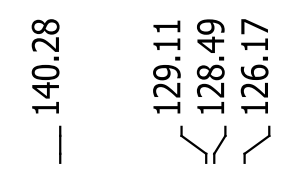
굴융
종

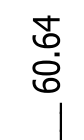

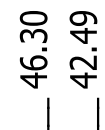

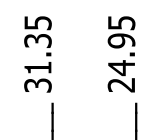

${ }^{13} \mathrm{C}-\mathrm{NMR}$ of $( \pm)-7 \mathbf{b}$ in $\mathrm{CDCl}_{3}$

${ }_{N} \lambda_{B n}$

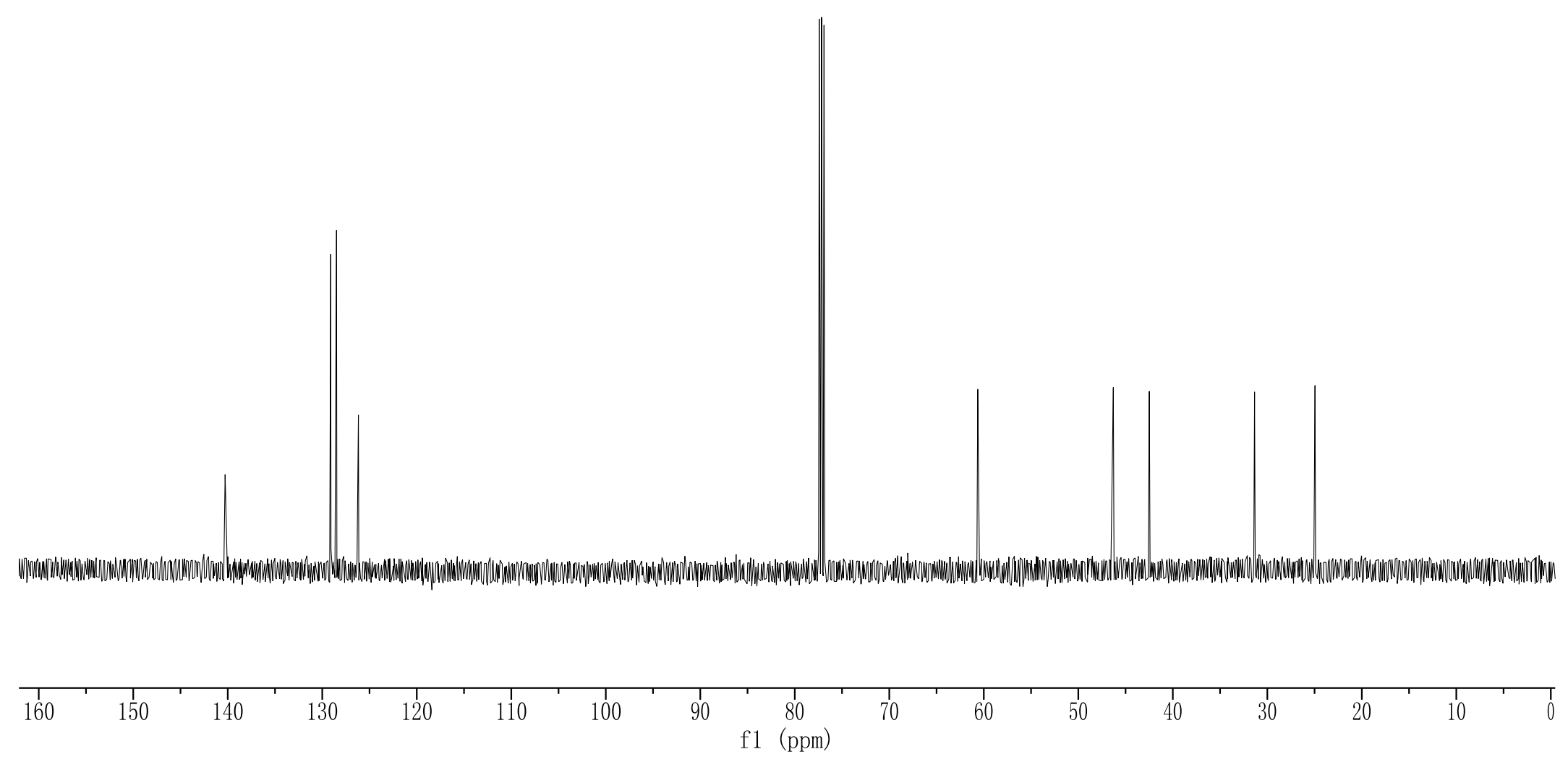




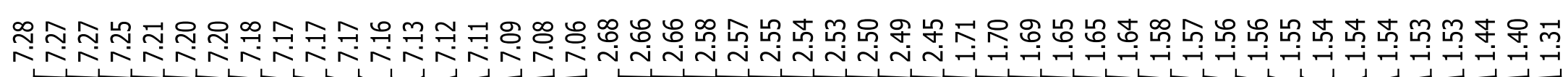
गाiाI

${ }^{1} \mathrm{H}-\mathrm{NMR}$ of $( \pm)-7 \mathbf{d}$ in $\mathrm{CDCl}_{3}$<smiles>BrCC(Br)C1CCCCN1</smiles>

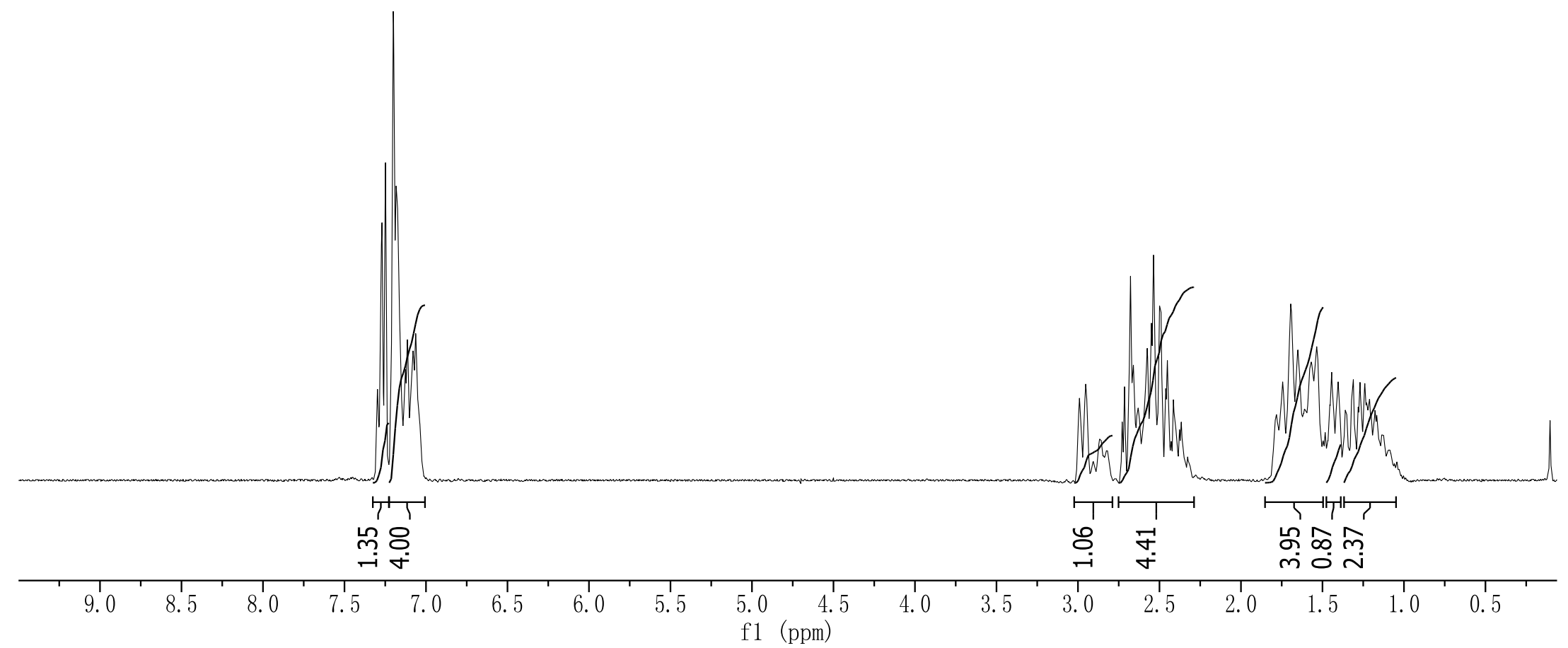




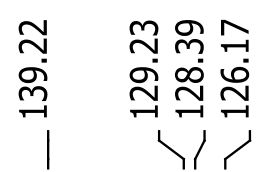

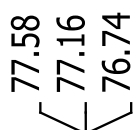

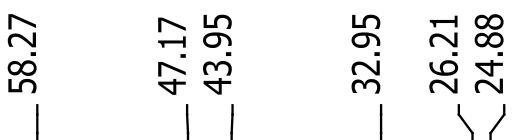

${ }^{13} \mathrm{C}-\mathrm{NMR}$ of $( \pm)-\mathbf{7 d}$ in $\mathrm{CDCl}_{3}$
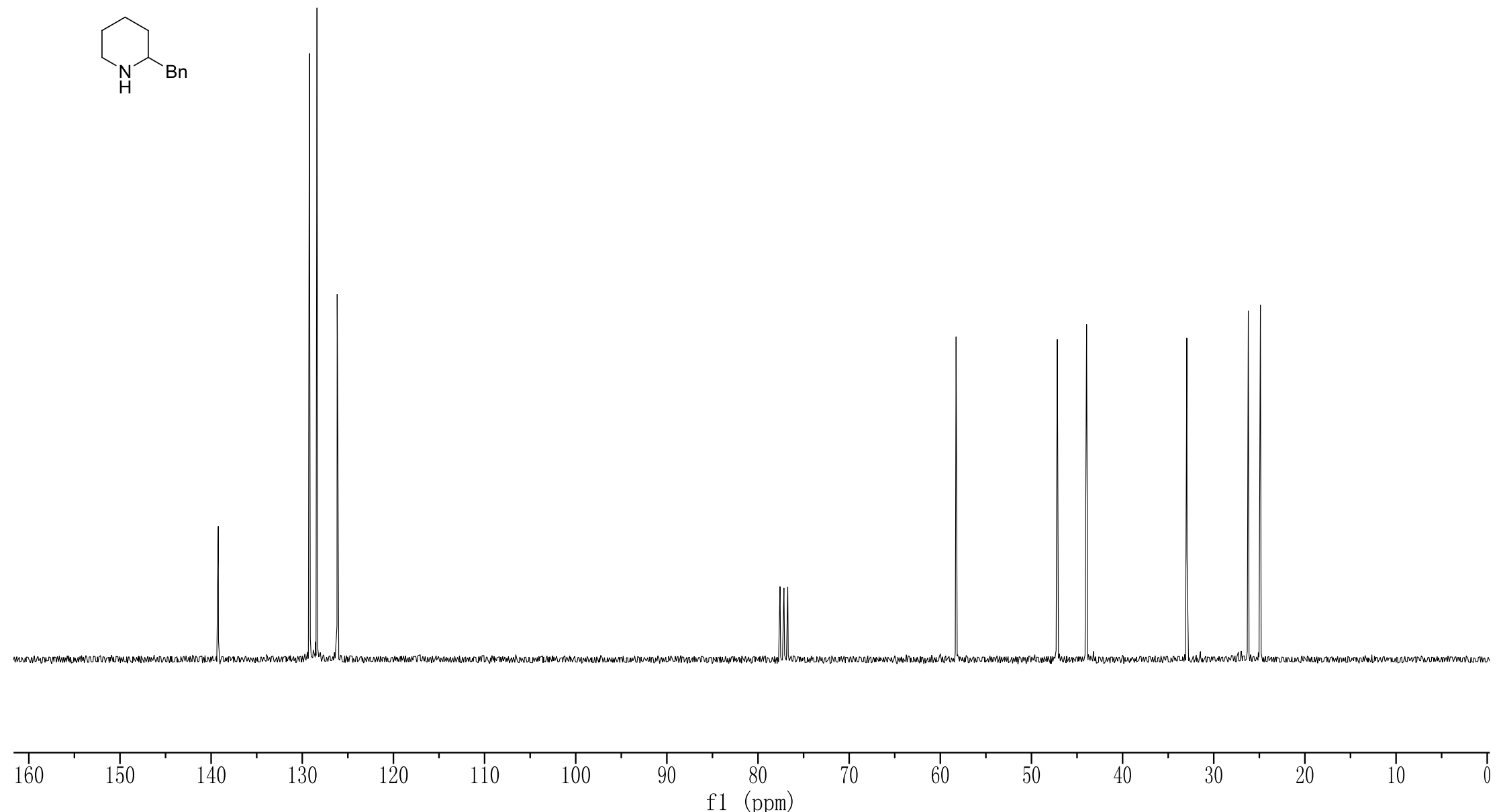


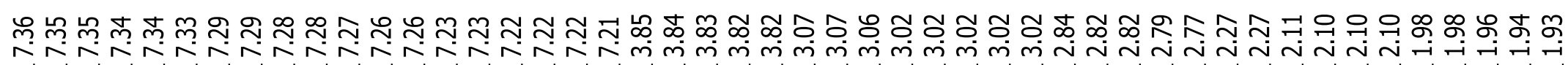

LLL L L L L L

${ }^{1} \mathrm{H}-\mathrm{NMR}$ of $( \pm)-7 e$ in $\mathrm{CDCl}_{3}$

$\mathrm{Me}$

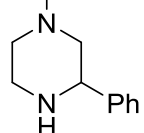

$\mathrm{H}$

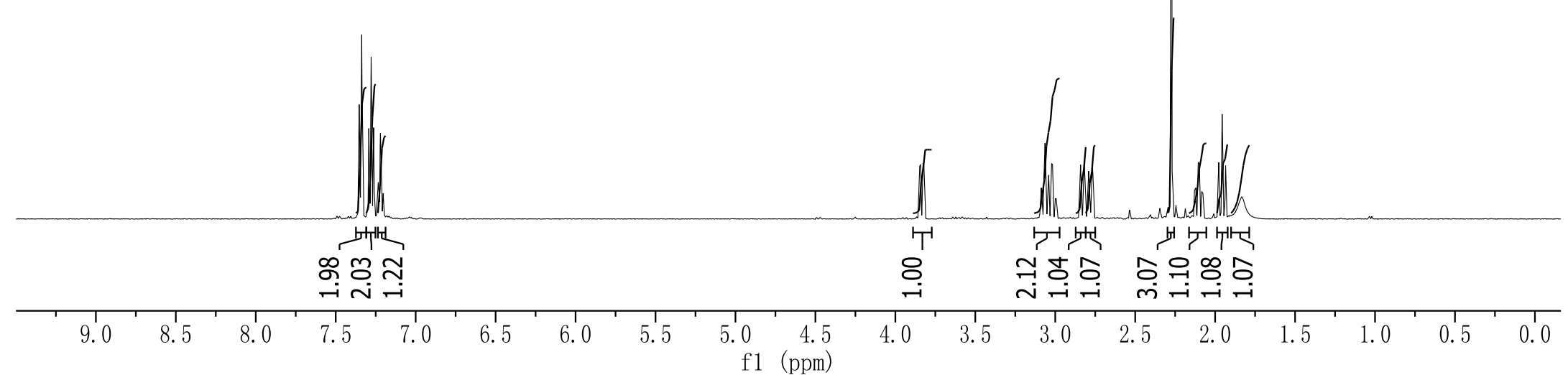



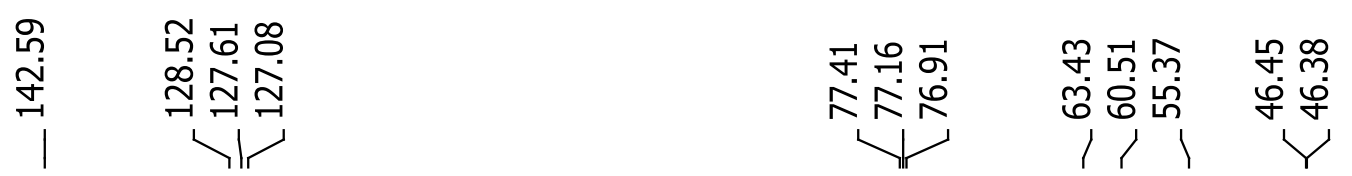

${ }^{13} \mathrm{C}-\mathrm{NMR}$ of $( \pm)-7 \mathrm{e}$ in $\mathrm{CDCl}_{3}$

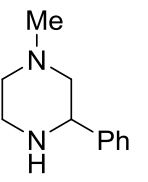

I.

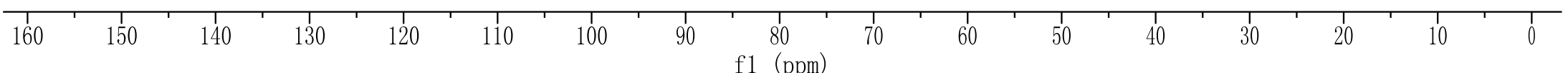


䒔 $\stackrel{L}{L}+1+$ III IIIIII

${ }^{1} \mathrm{H}-\mathrm{NMR}$ of $( \pm)-7 \mathrm{f}$ in $\mathrm{CDCl}_{3}$<smiles>c1ccc(CC2CCCCCN2)cc1</smiles>

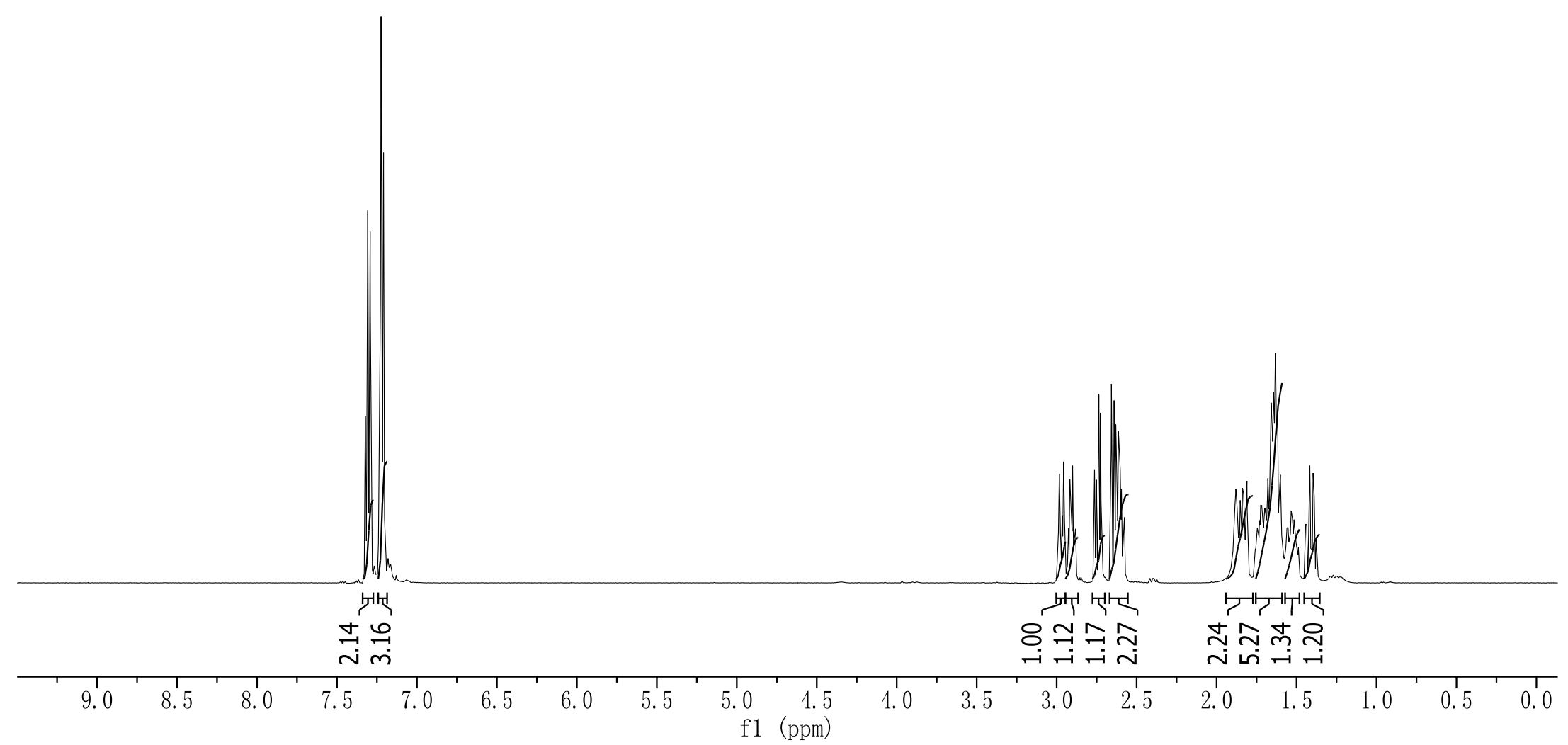




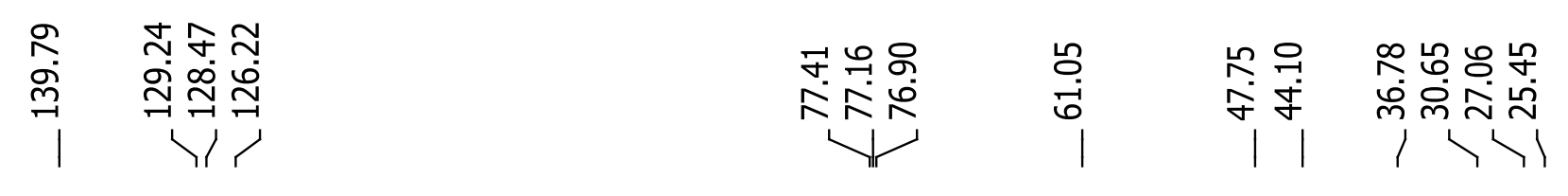

${ }^{13} \mathrm{C}-\mathrm{NMR}$ of $( \pm)-7 \mathrm{f}$ in $\mathrm{CDCl}_{3}$
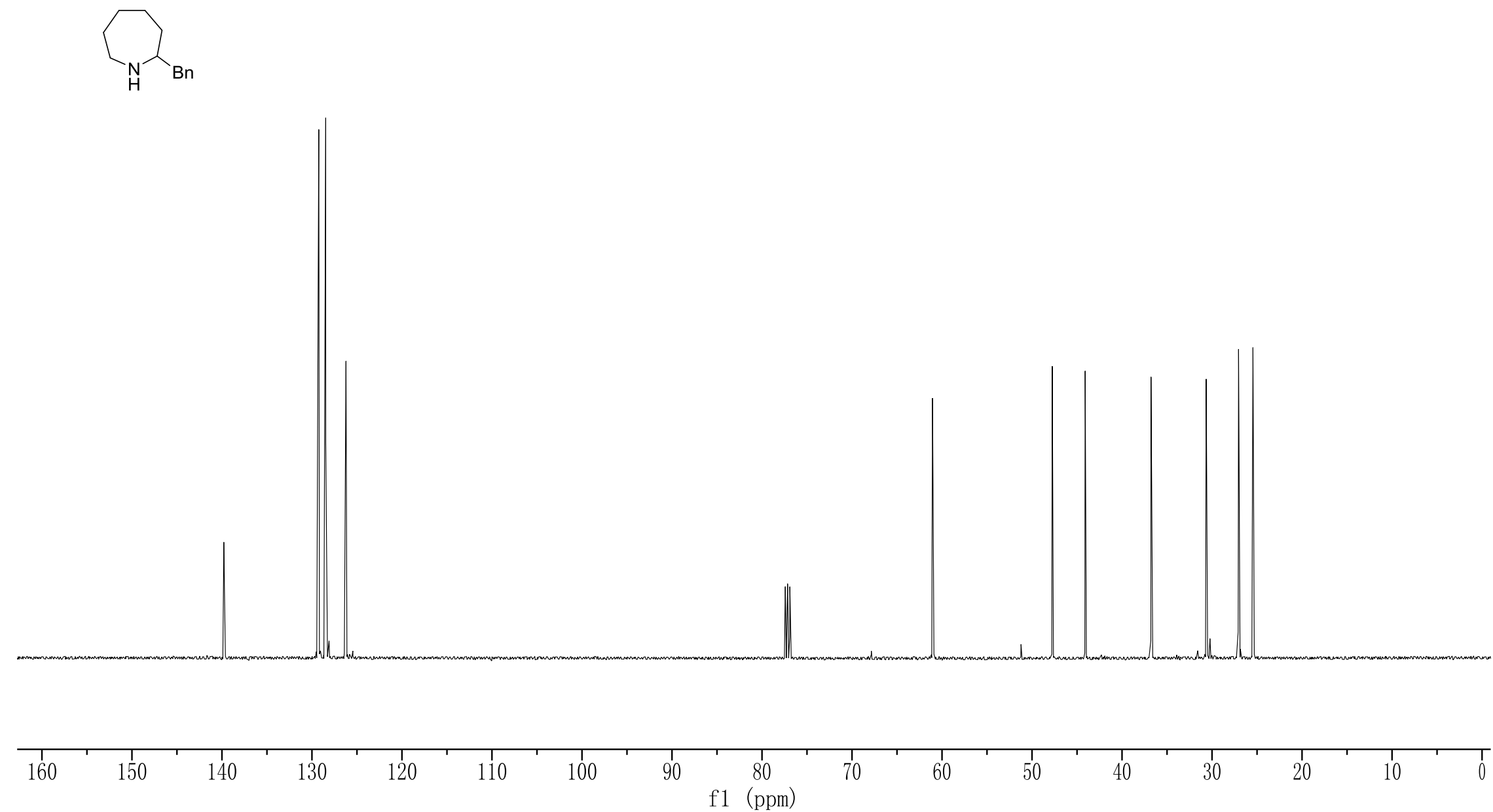


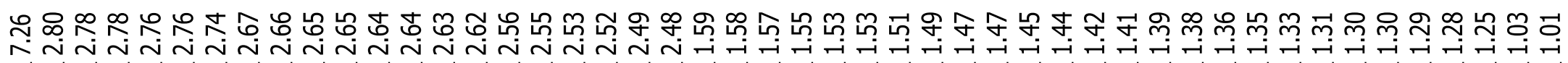
N $N$, $N$, $N$,

${ }^{1} \mathrm{H}-\mathrm{NMR}$ of $( \pm)-\mathbf{7 g}$ in $\mathrm{CDCl}_{3}$

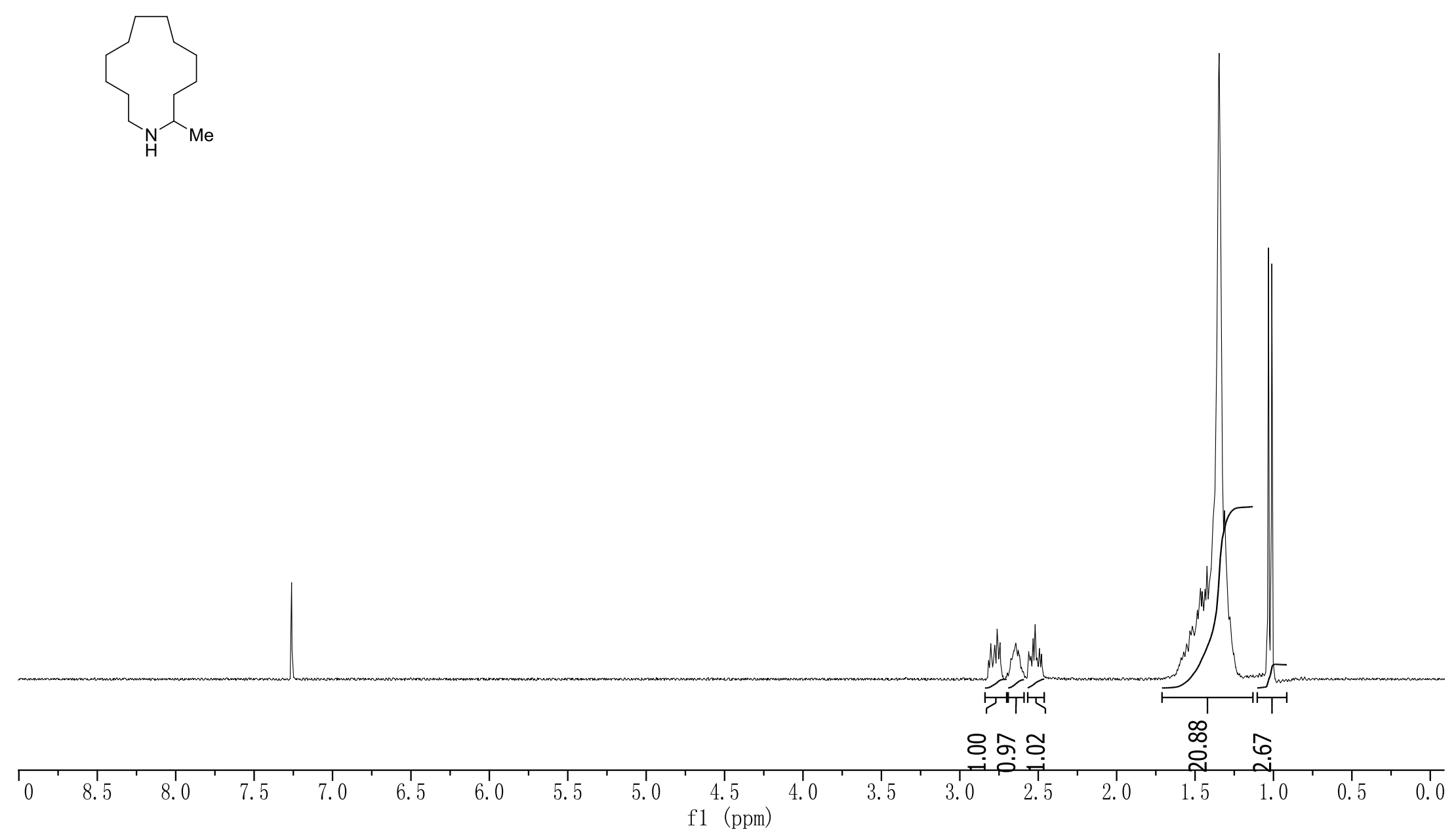

IIIIIIIIIIII) 
${ }^{13} \mathrm{C}-\mathrm{NMR}$ of $( \pm)-\mathbf{7 g}$ in $\mathrm{CDCl}_{3}$

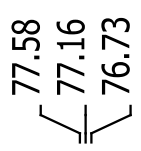

กับ เุ

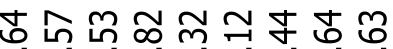

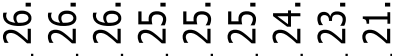

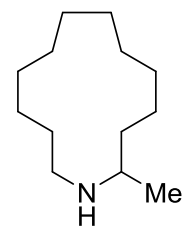

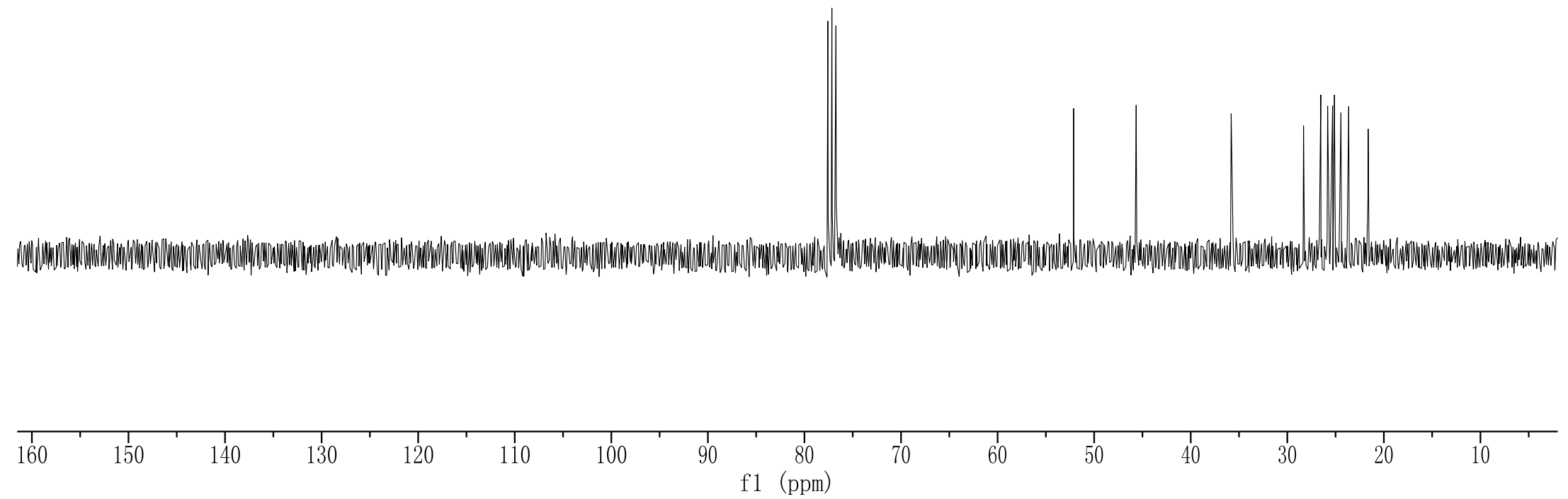




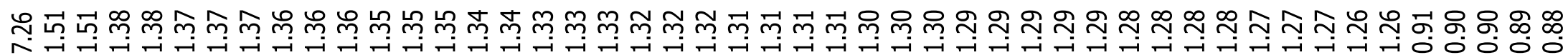

${ }^{1} \mathrm{H}-\mathrm{NMR}$ of $( \pm)-7 \mathrm{~h}$ in $\mathrm{CDCl}$
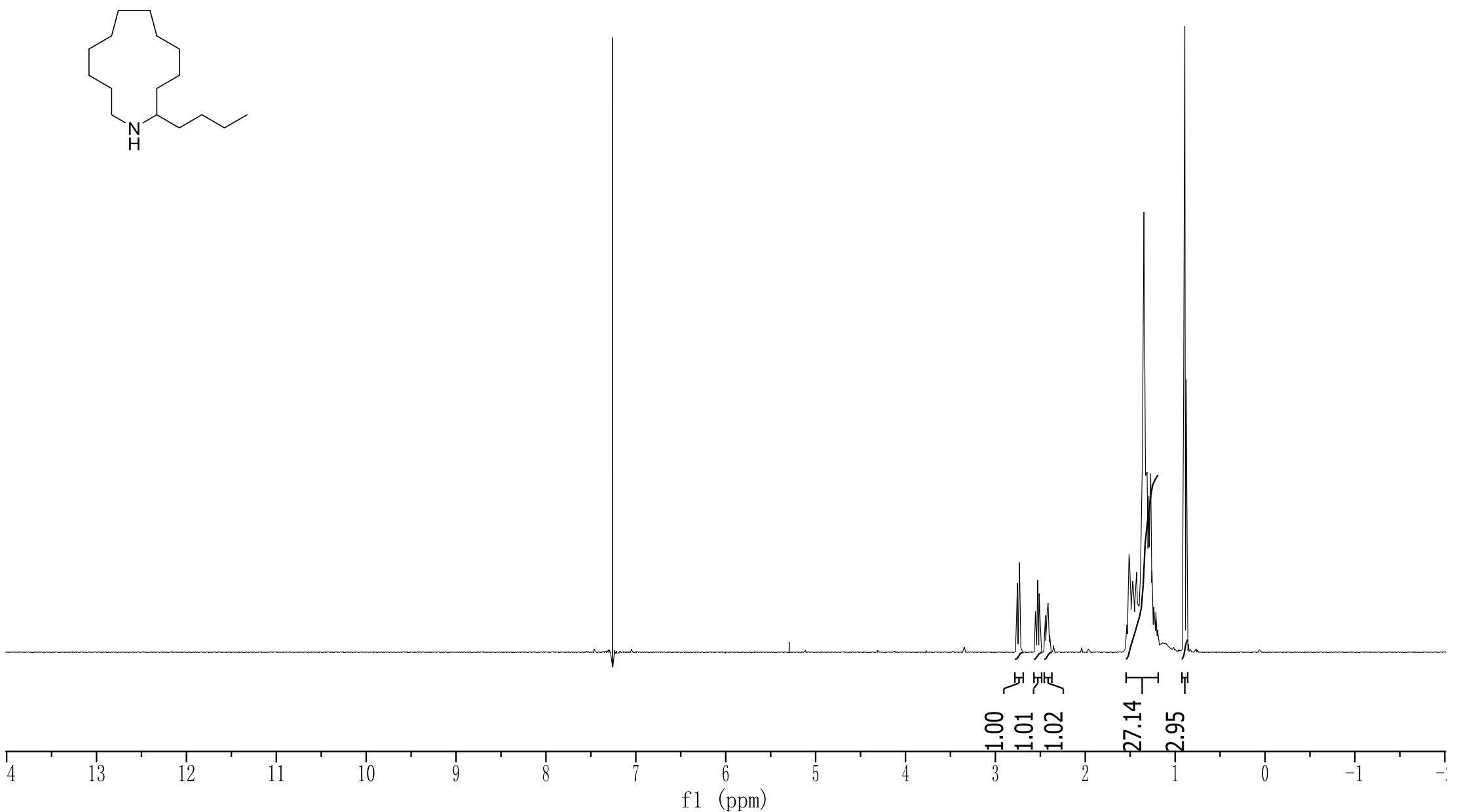


$$
\begin{aligned}
& \text { 하윰어 } \\
& \text { ㅊํㅇ } \\
& 4
\end{aligned}
$$

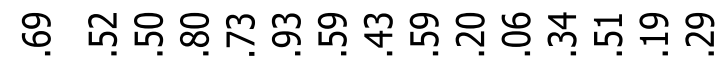

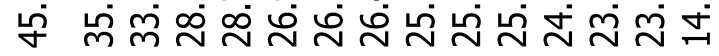

${ }^{13} \mathrm{C}-\mathrm{NMR}$ of $( \pm)-7 \mathbf{h}$ in $\mathrm{CDCl}_{3}$
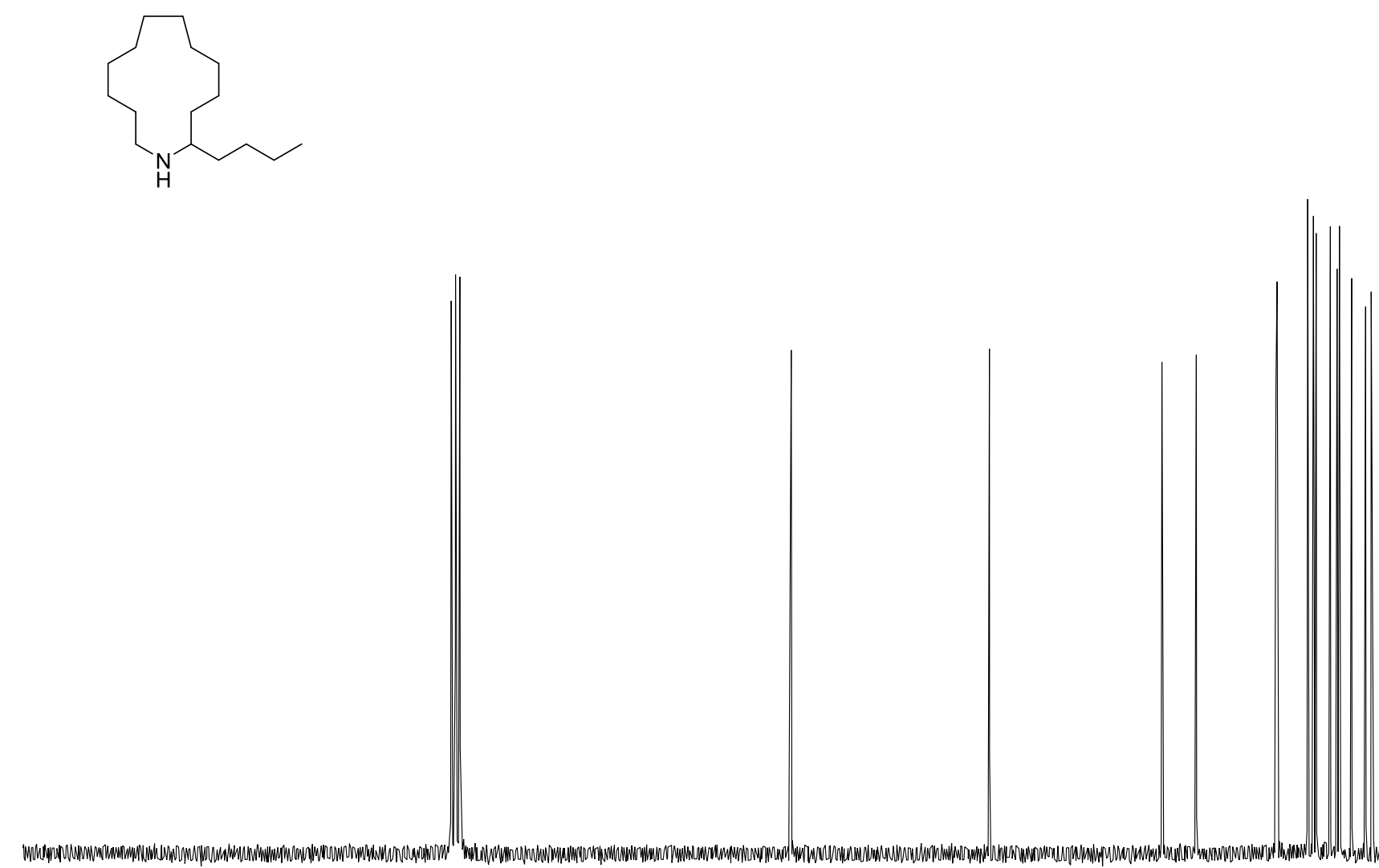

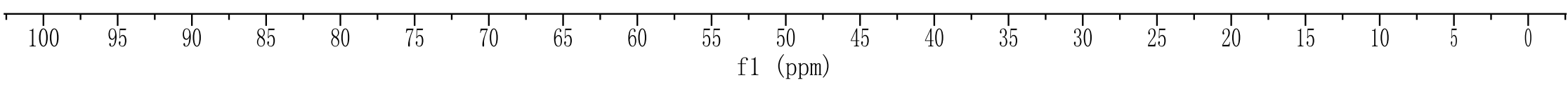




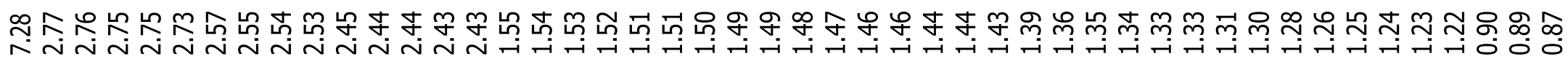

L

${ }^{1} \mathrm{H}-\mathrm{NMR}$ of $( \pm)-7 \mathbf{i}$ in $\mathrm{CDCl}_{3}$
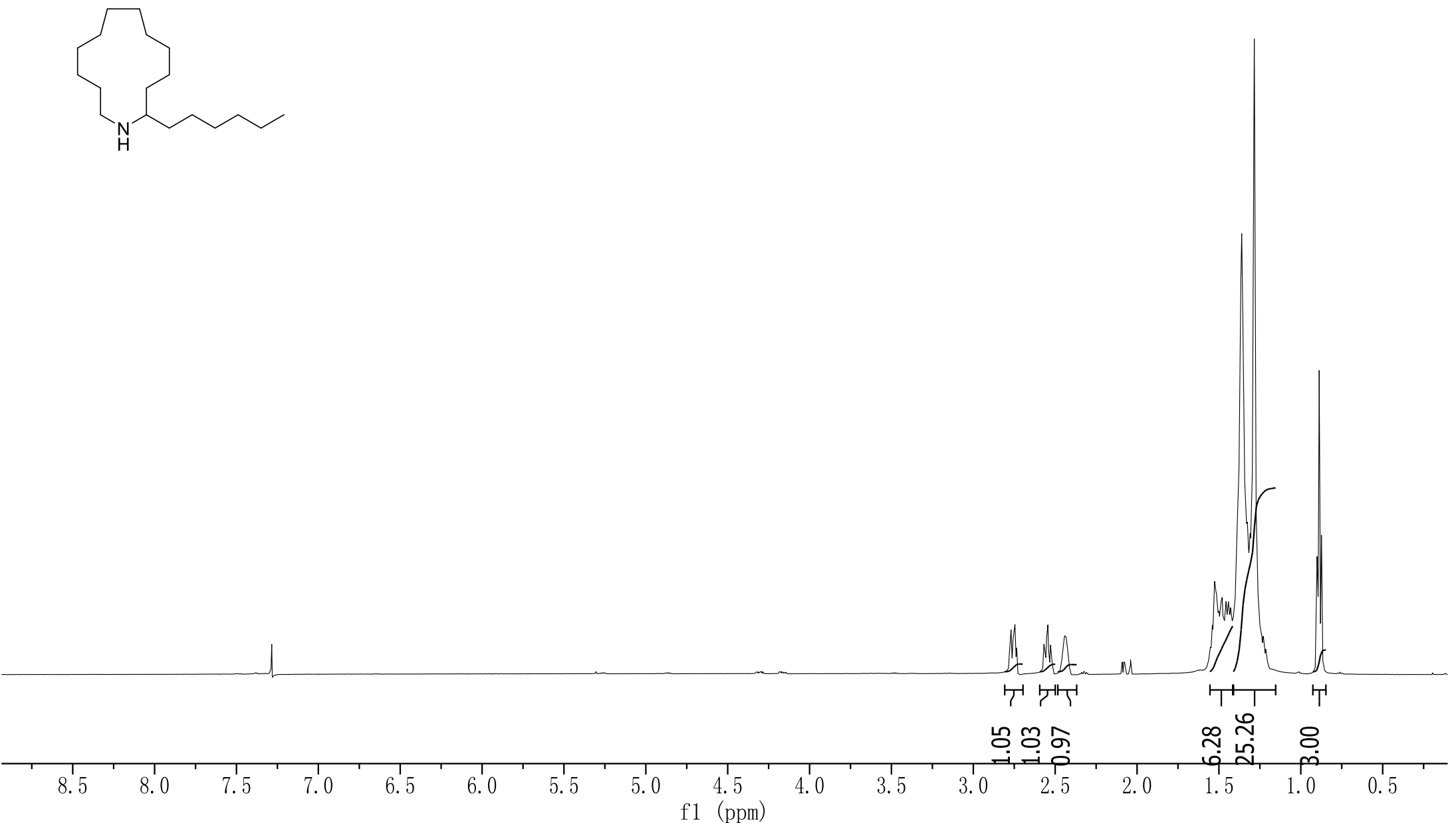


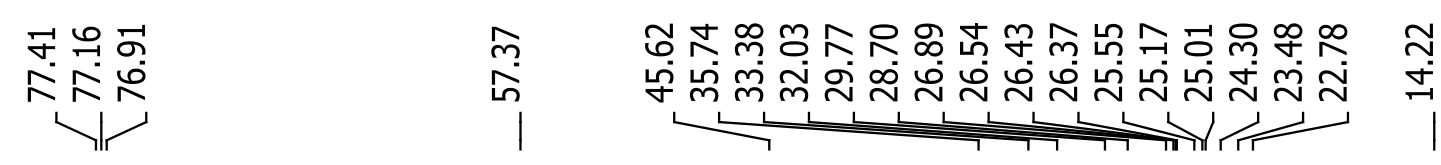

${ }^{13} \mathrm{C}-\mathrm{NMR}$ of $( \pm)-7 \mathbf{i}$ in $\mathrm{CDCl}_{3}$
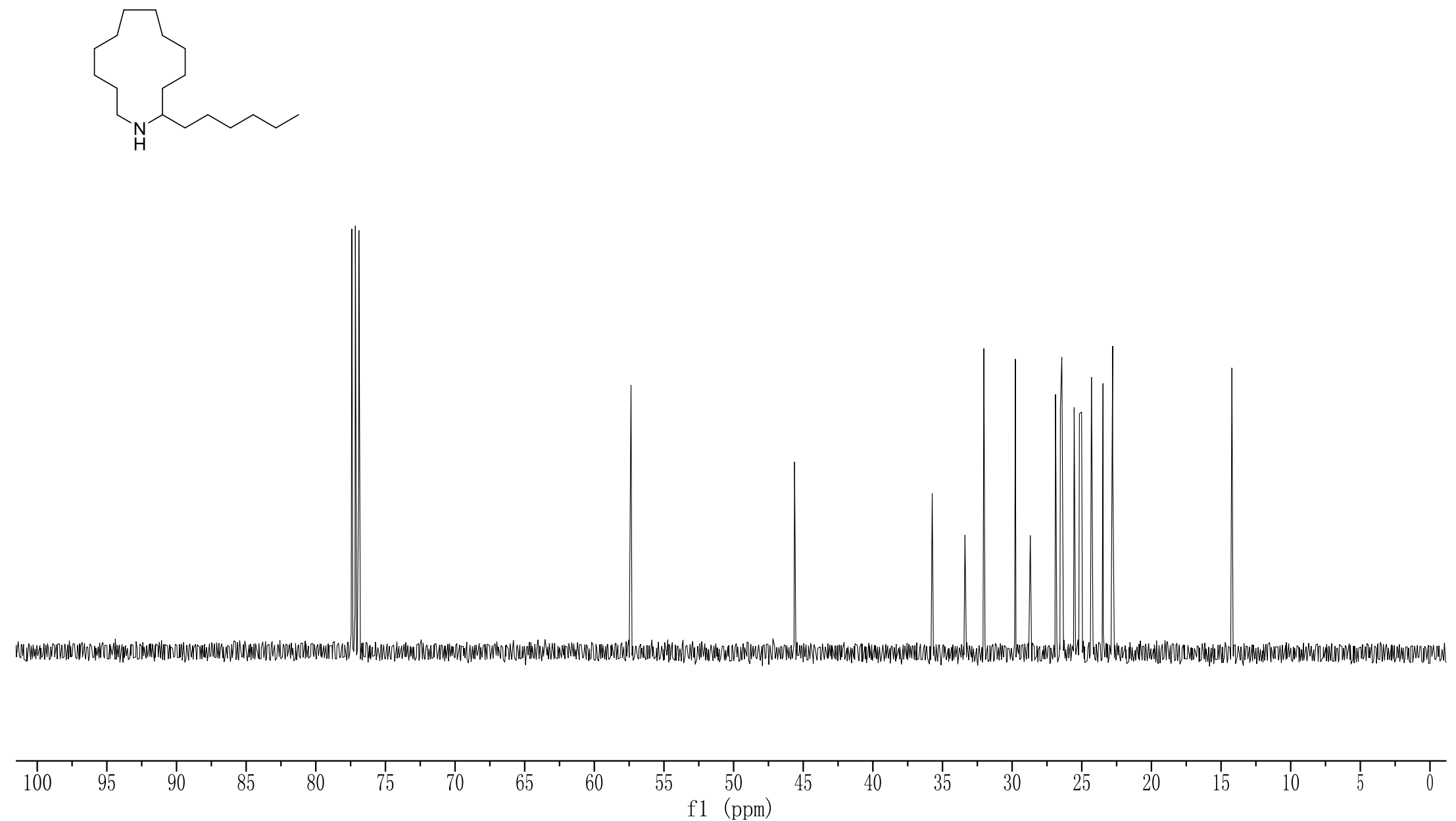


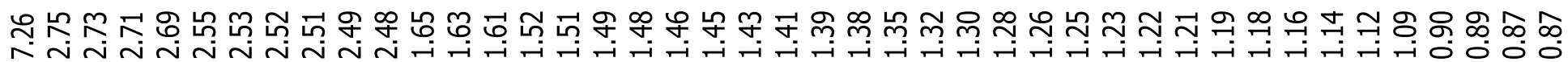

${ }^{1} \mathrm{H}-\mathrm{NMR}$ of $( \pm)-7 \mathrm{j}$ in $\mathrm{CDCl}_{3}$

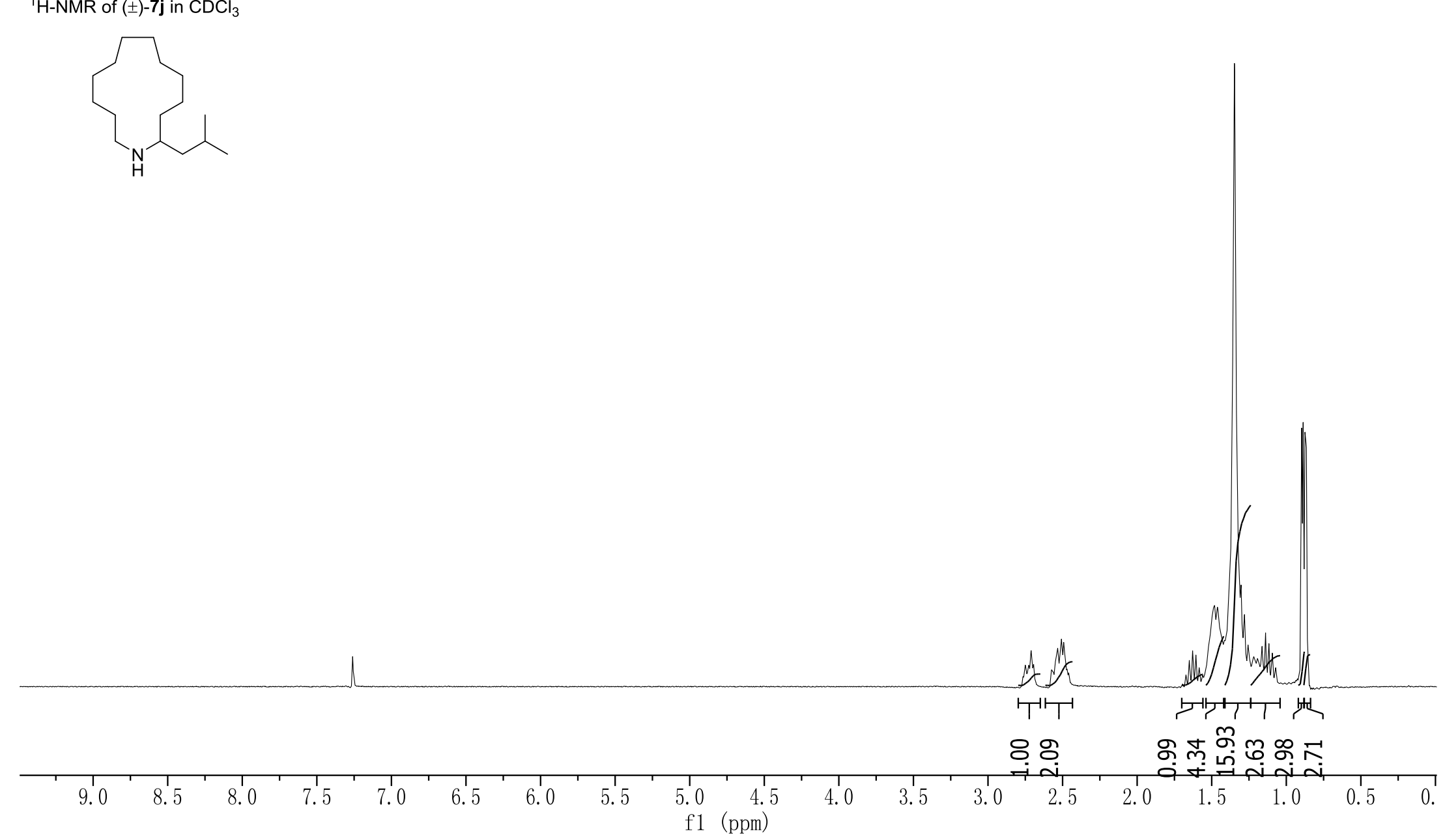

III 


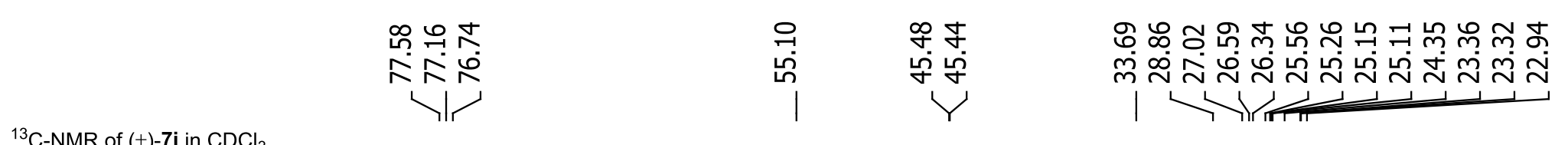

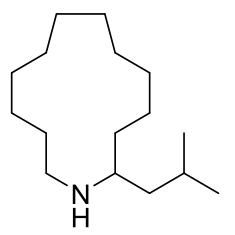

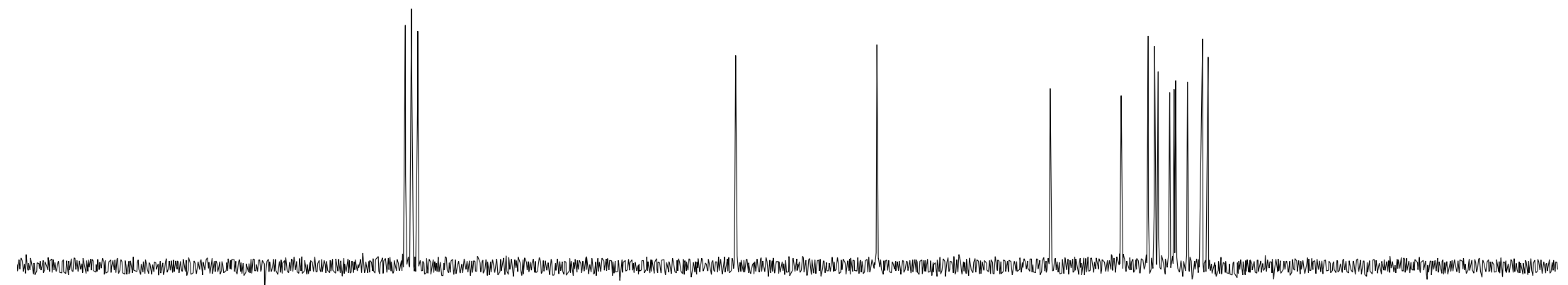

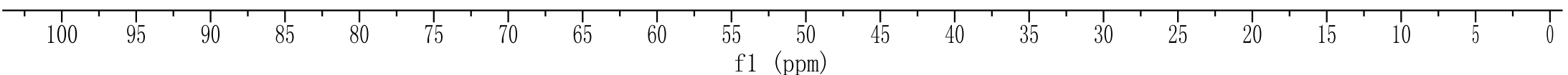




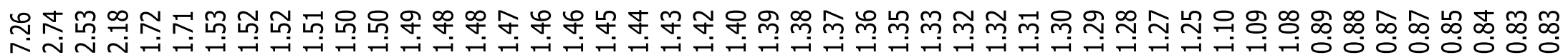

${ }^{1} \mathrm{H}-\mathrm{NMR}$ of $( \pm)-7 \mathbf{k}$ in $\mathrm{CDCl}_{3}$
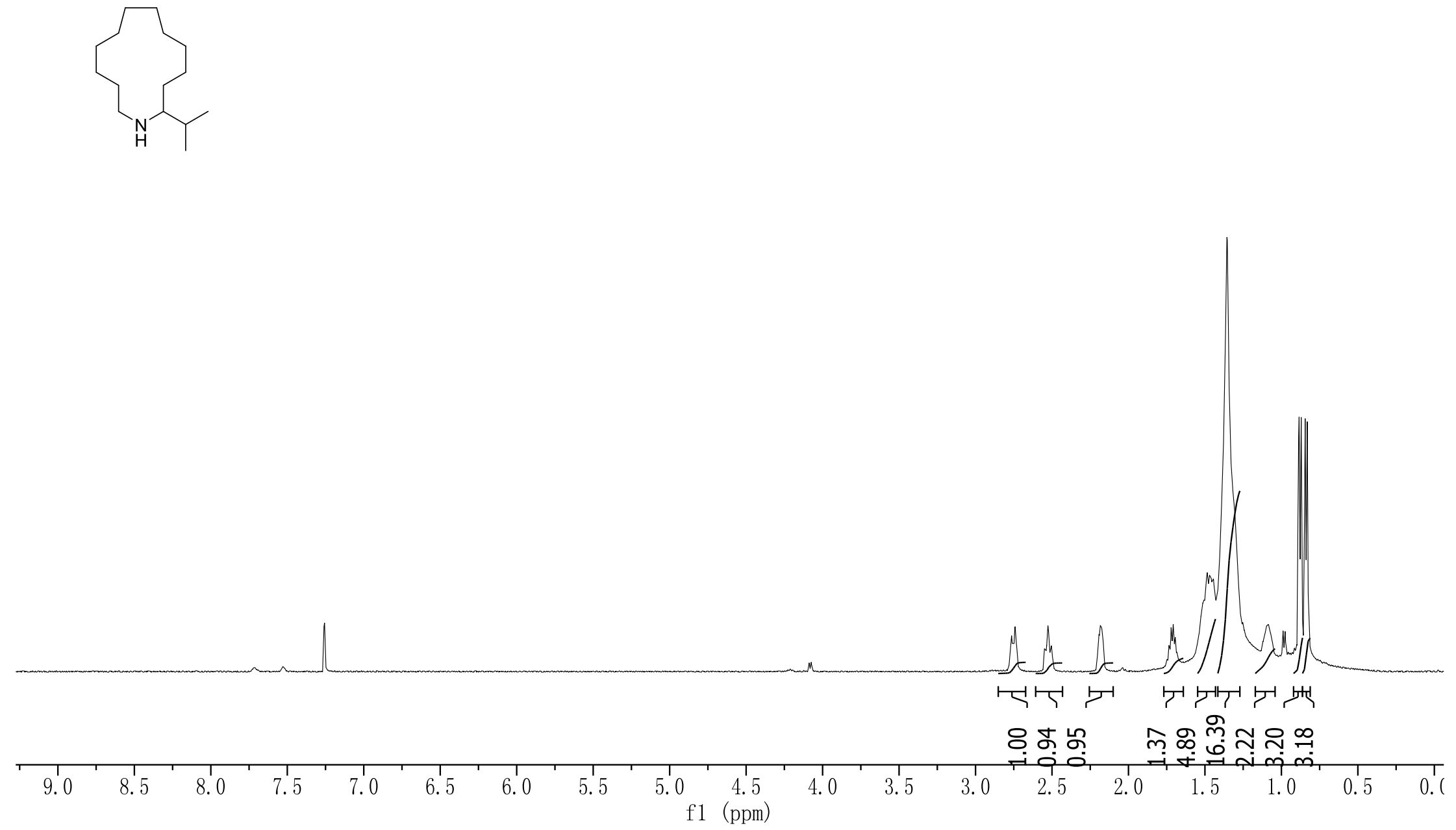


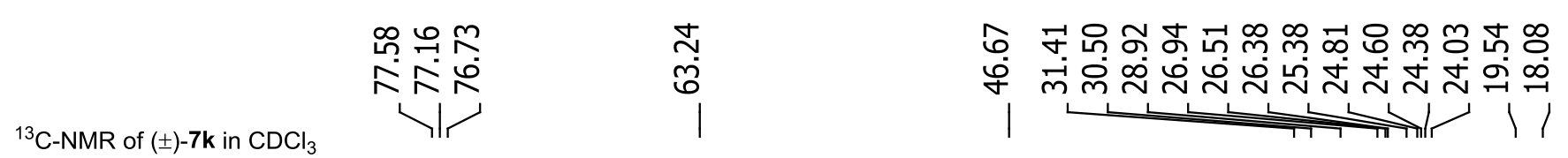

. Im

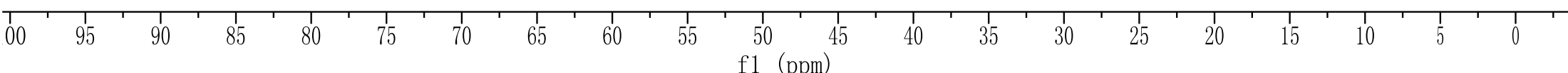




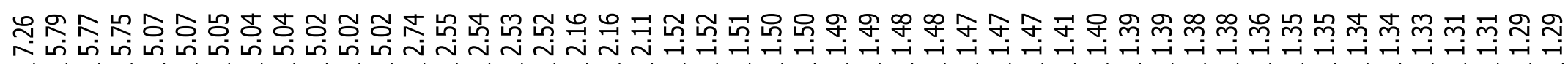

ـ L L

${ }^{1} \mathrm{H}-\mathrm{NMR}$ of $( \pm)-7 \mathrm{I}$ in $\mathrm{CDCl}_{3}$
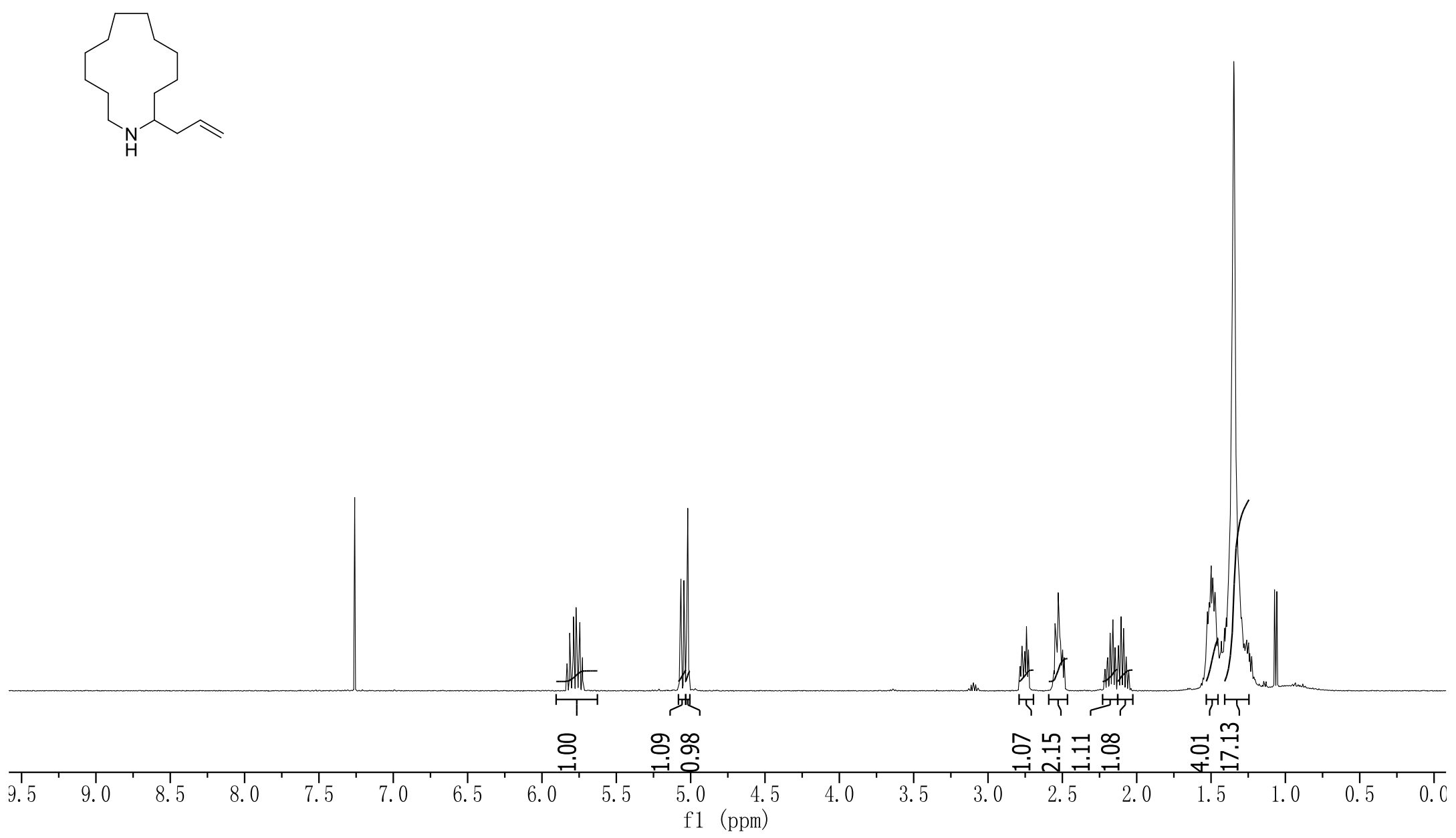


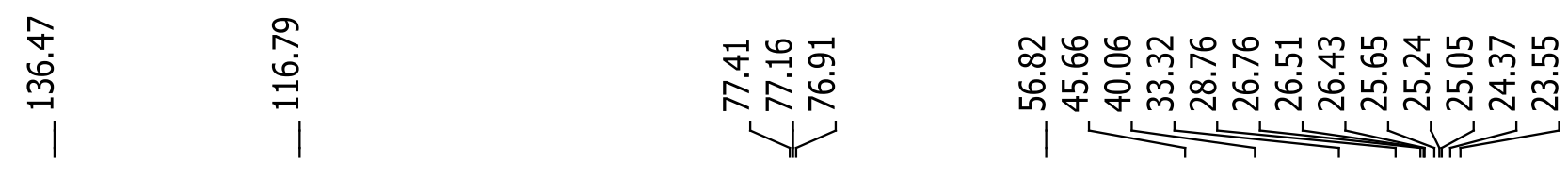

${ }^{13} \mathrm{C}-\mathrm{NMR}$ of $( \pm)-7 \mathrm{I}$ in $\mathrm{CDCl}_{3}$
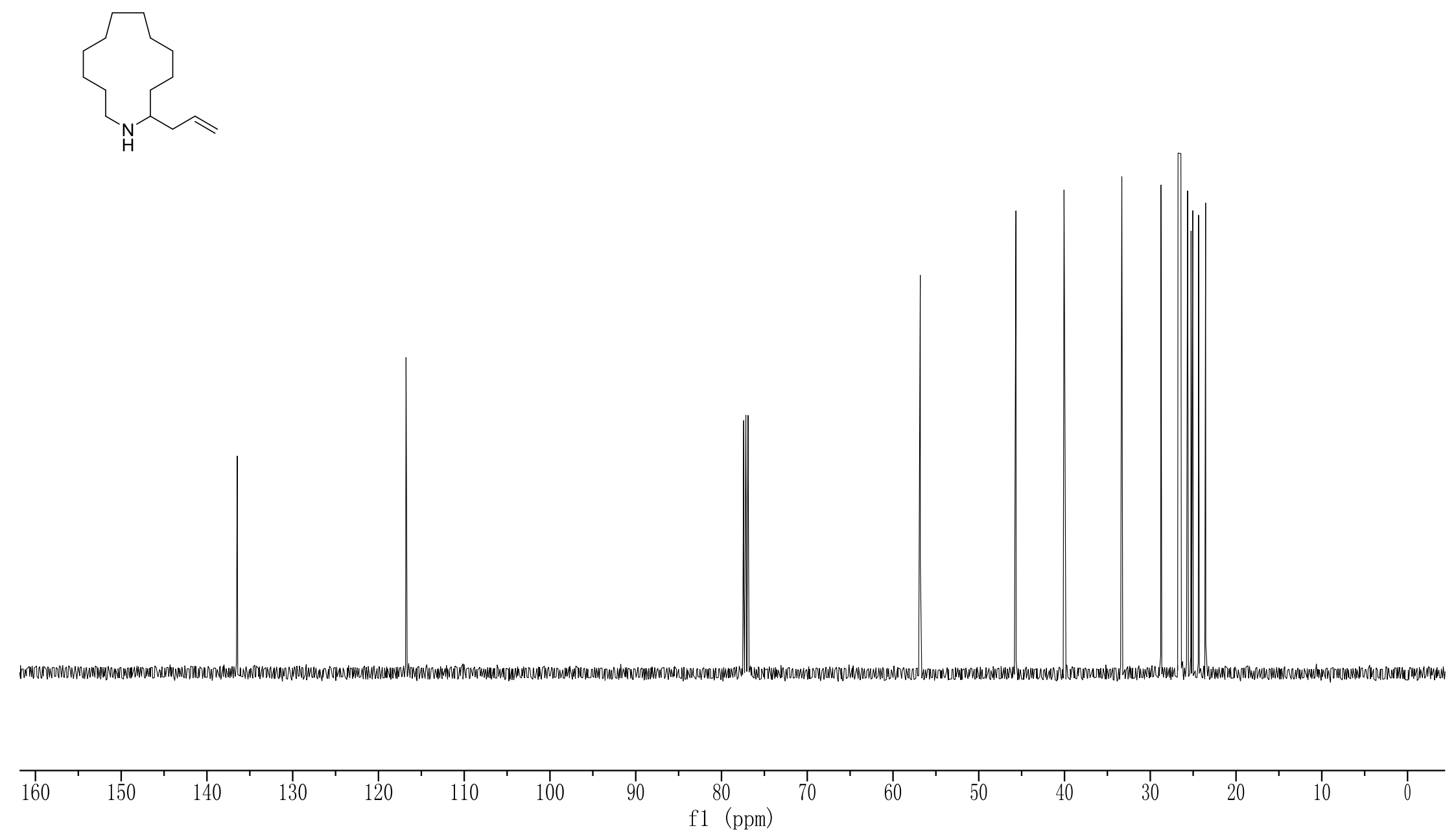


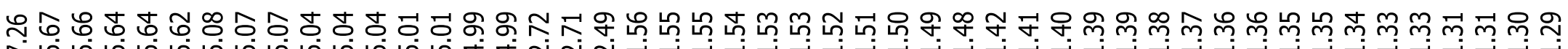

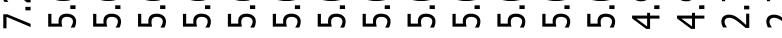

${ }^{1} \mathrm{H}-\mathrm{NMR}$ of $( \pm)-7 \mathrm{~m}$ in $\mathrm{CDCl}_{3}$

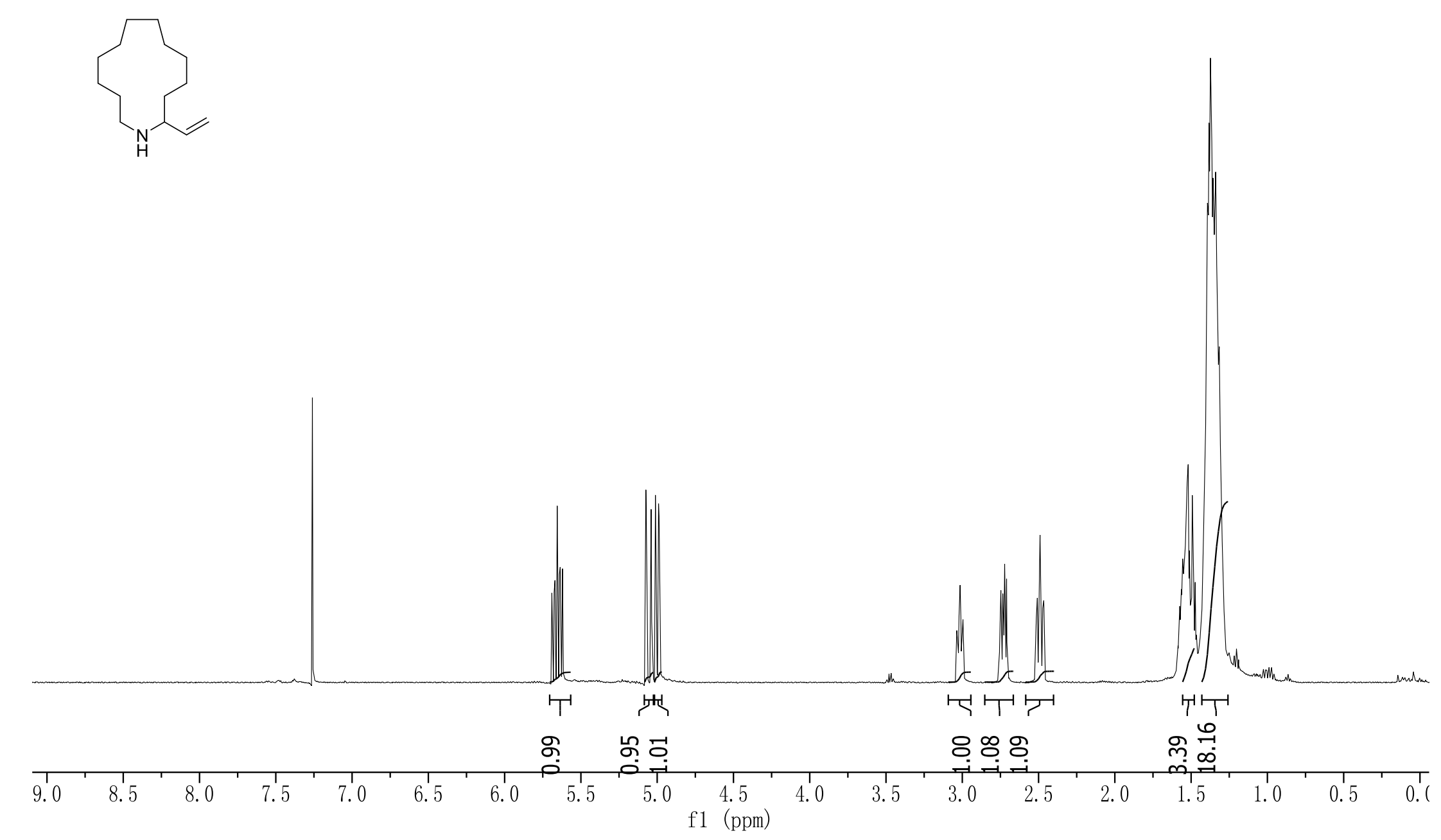

7mI 


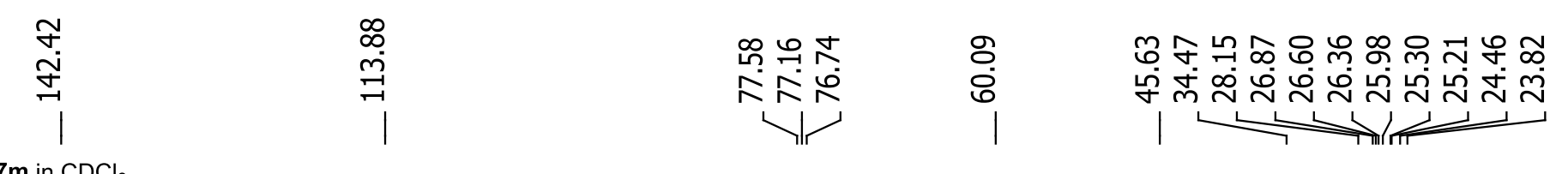

${ }^{13} \mathrm{C}-\mathrm{NMR}$ of $( \pm)-7 \mathrm{~m}$ in $\mathrm{CDCl}_{3}$

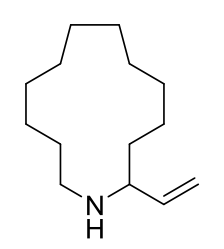

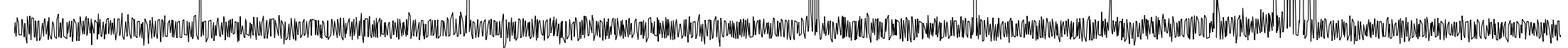

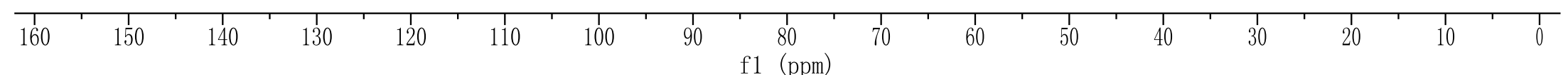




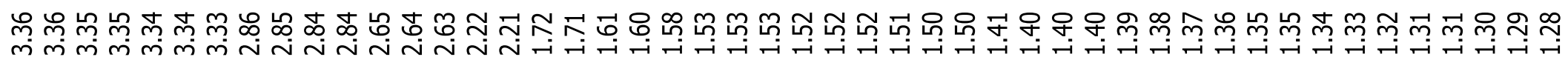

${ }^{1} \mathrm{H}-\mathrm{NMR}$ of $( \pm)-7 \mathbf{n}$ in $\mathrm{CDCl}_{3}$
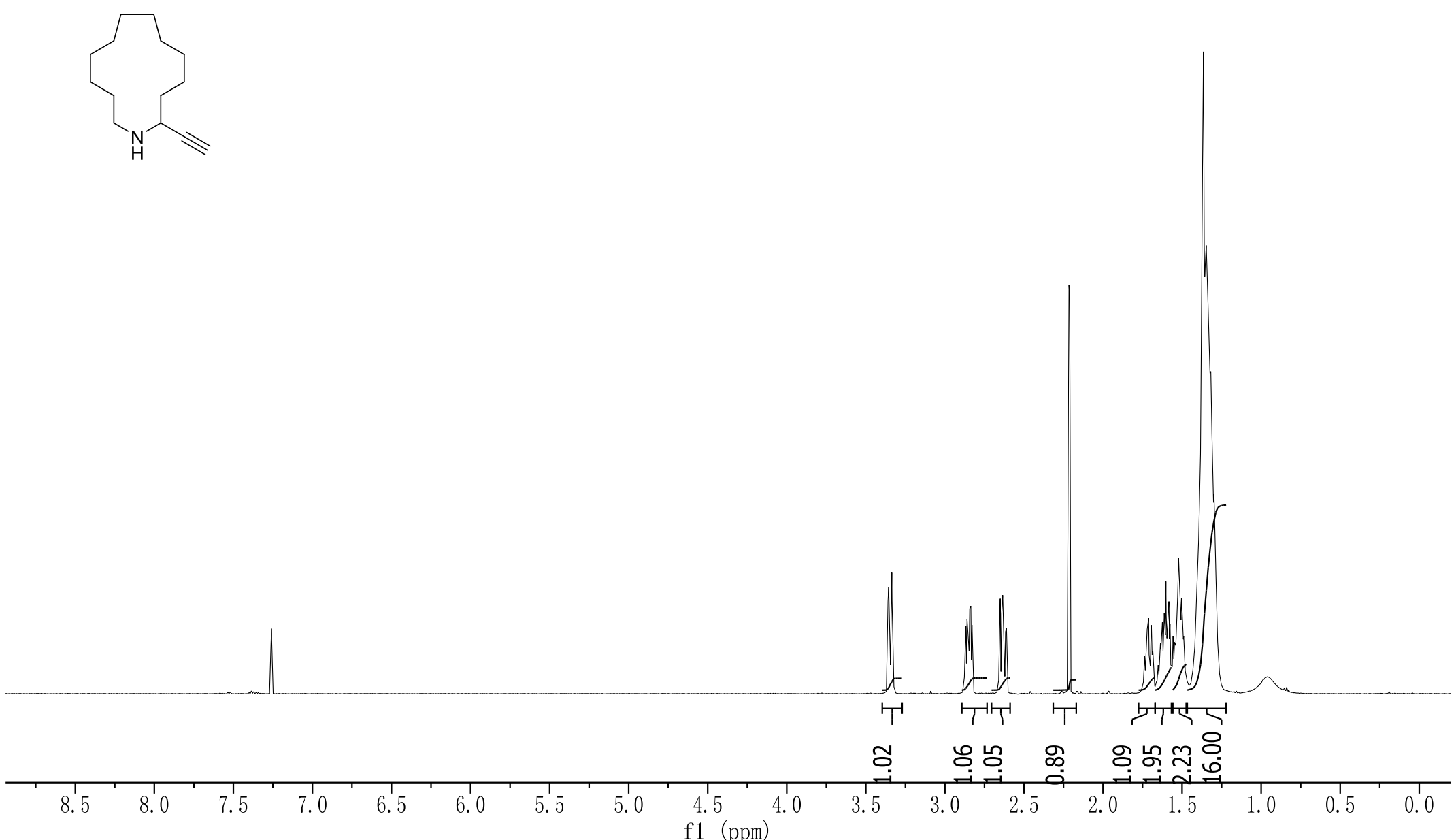
${ }^{13} \mathrm{C}-\mathrm{NMR}$ of $( \pm)-\mathbf{7 n}$ in $\mathrm{CDCl}_{3}$
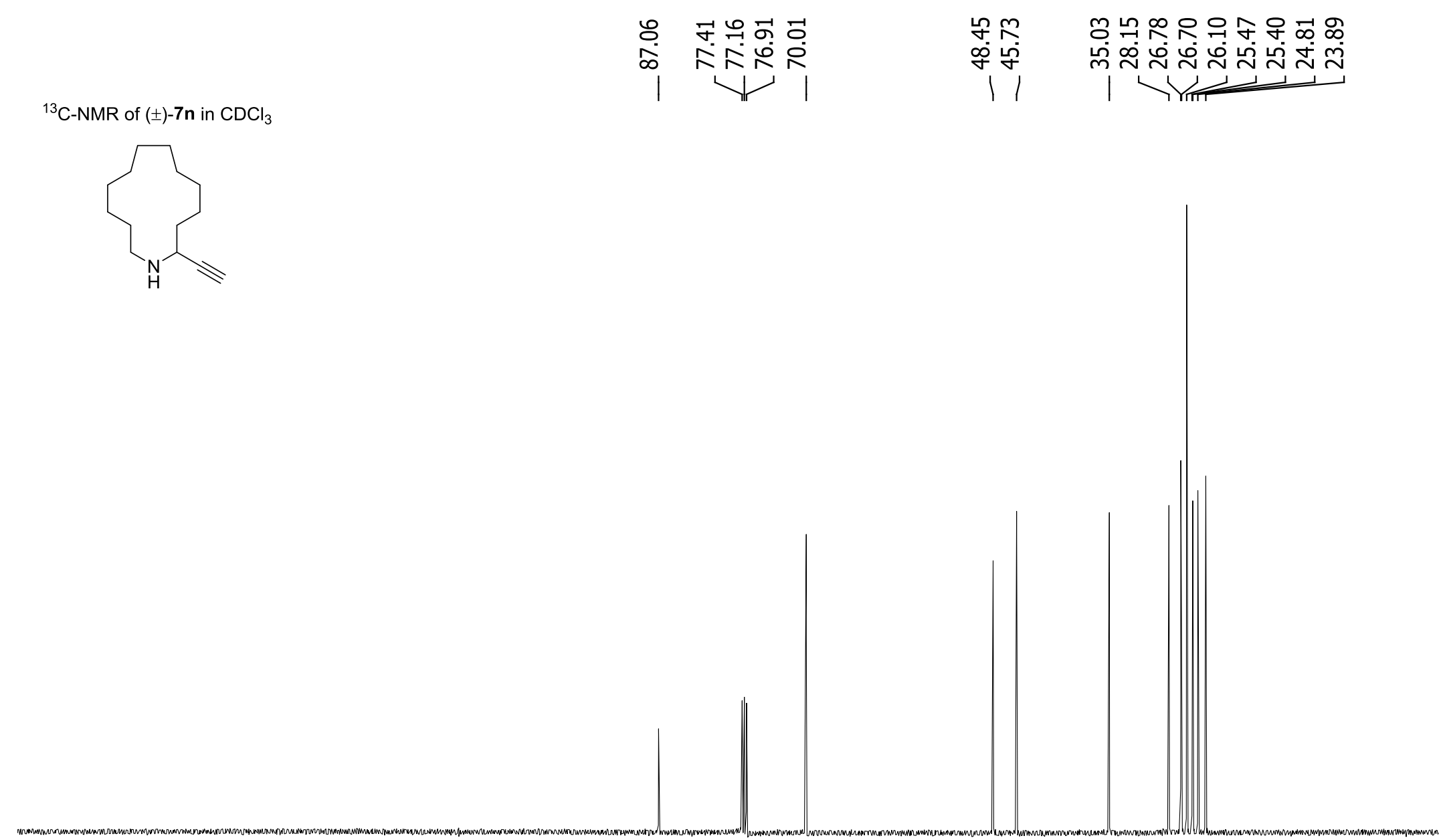

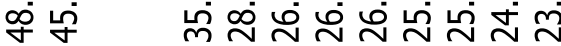

0

पाI

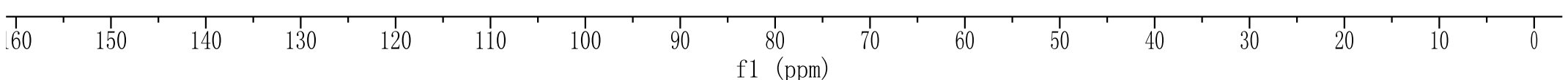




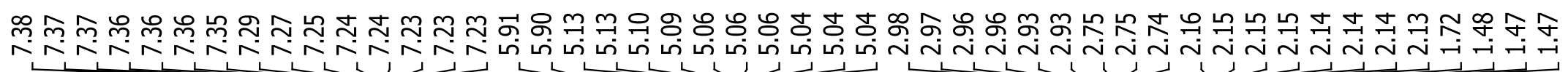

烈

${ }^{1} \mathrm{H}-\mathrm{NMR}$ of $( \pm)-7 \mathrm{p}$ in $\mathrm{CDCl}_{3}$

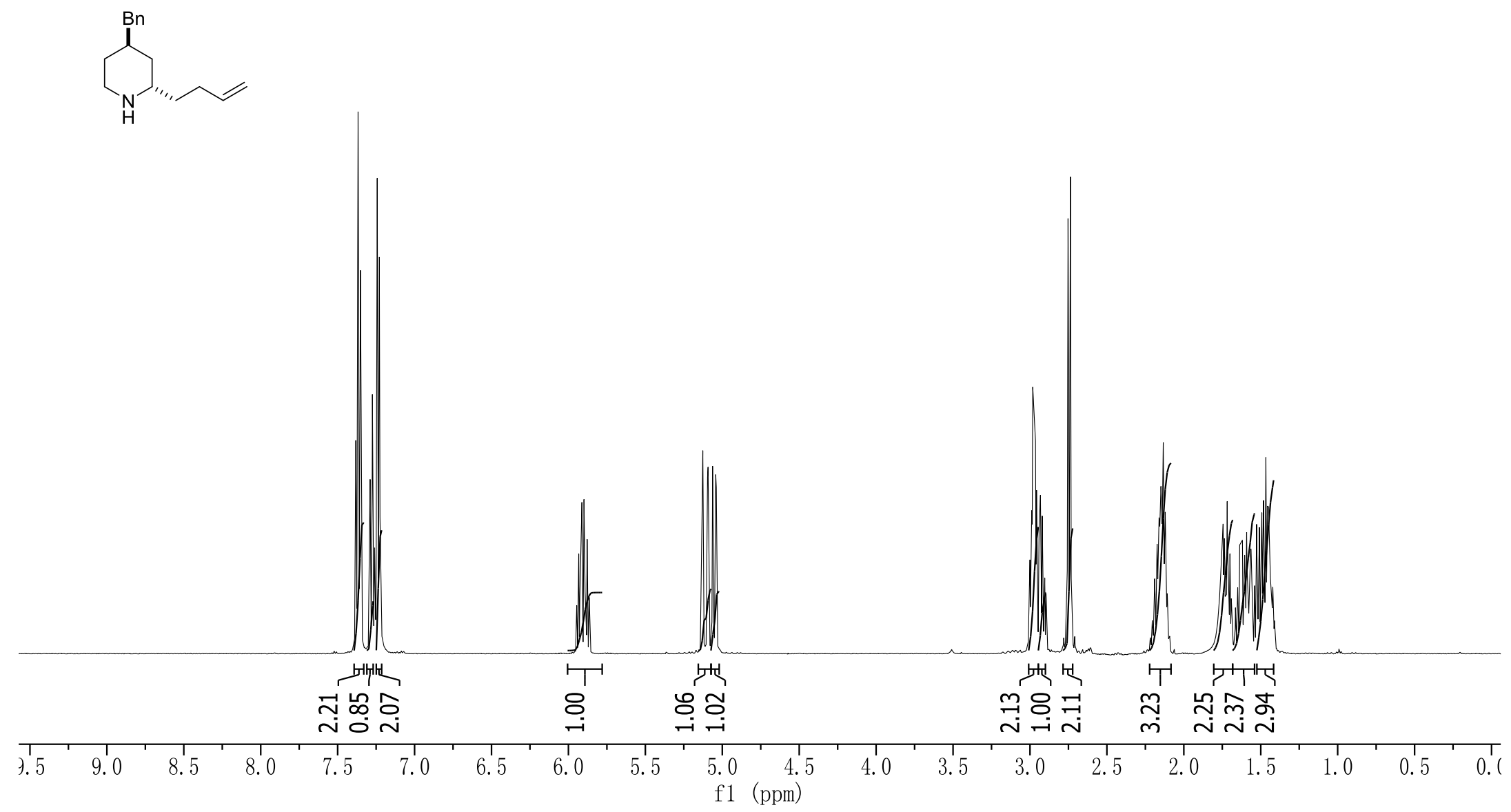



|

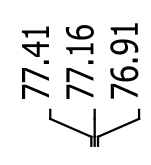

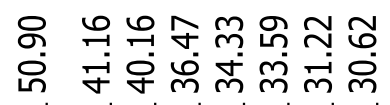
ம
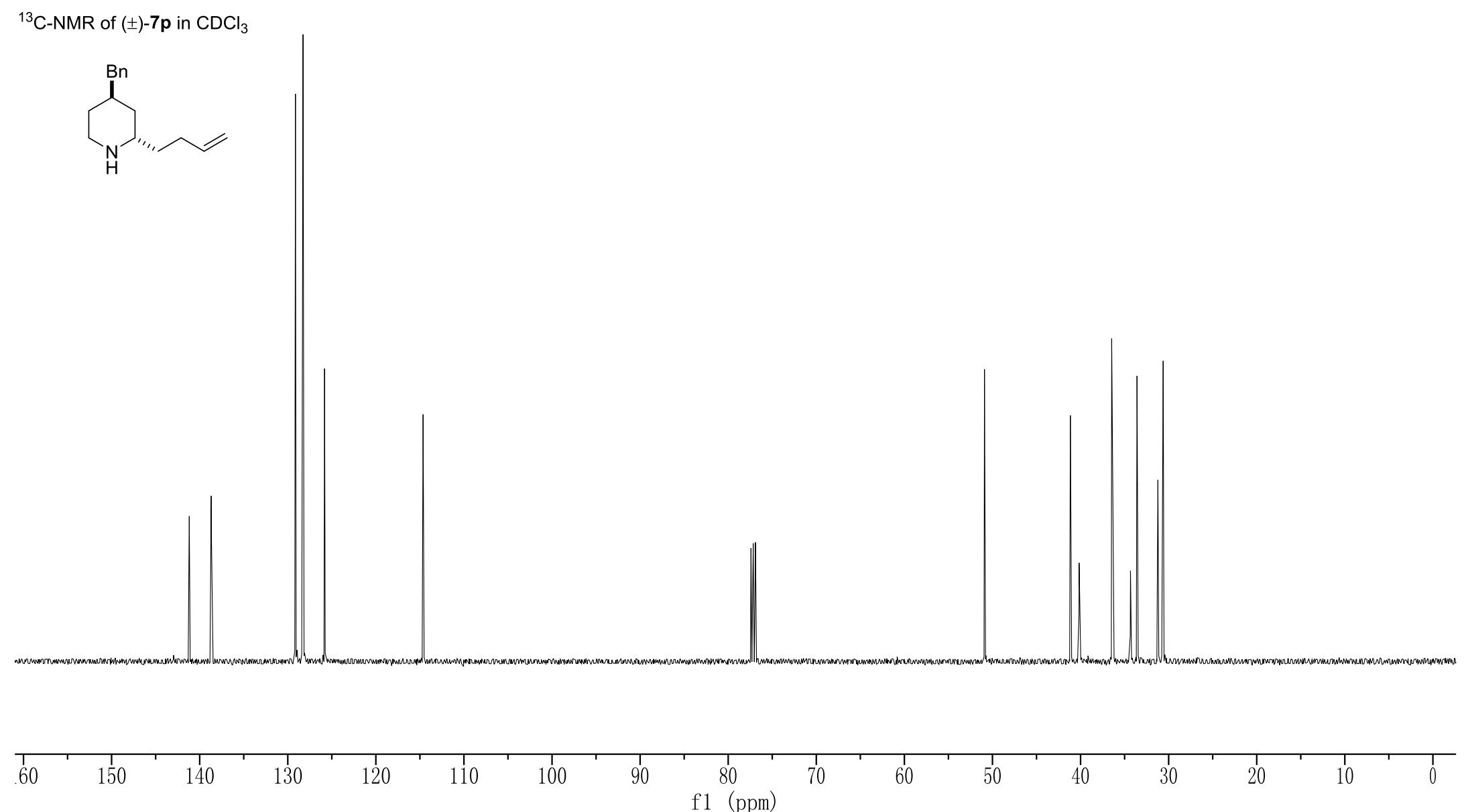


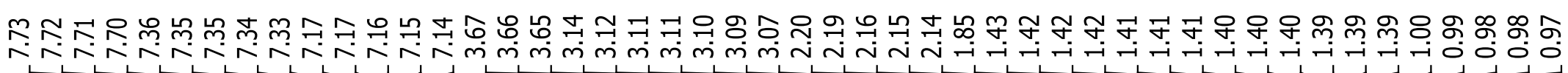

${ }^{1} \mathrm{H}-\mathrm{NMR}$ of $( \pm)-7 \mathbf{q}$ in $\mathrm{CDCl}_{3}$
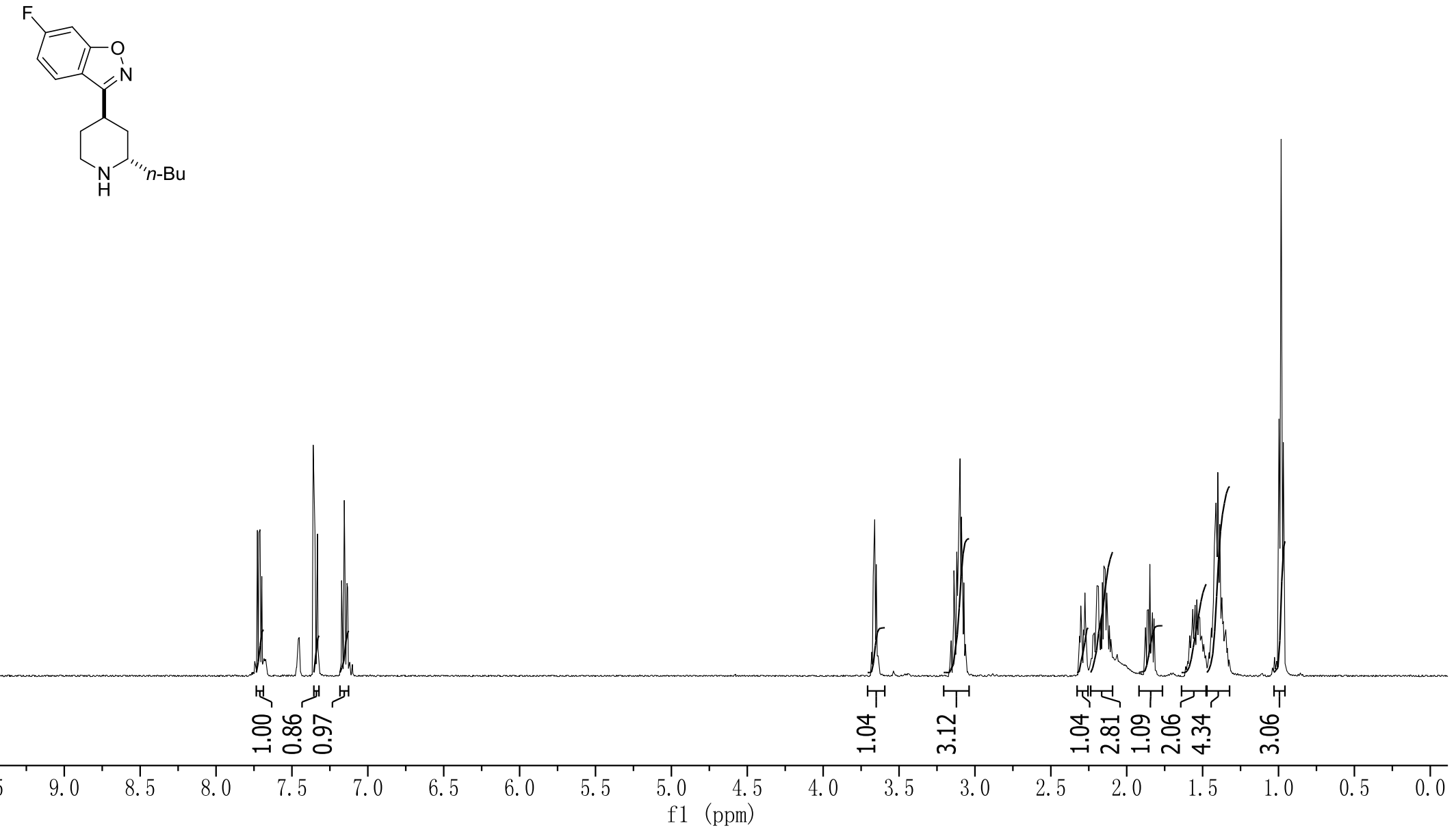
규ำํํㅇ

엉 ف잉

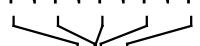

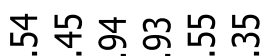

ก่งกำ

콬ㅋㅋㅋㅋㅋㅎㅇ

पY $Y$

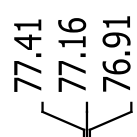

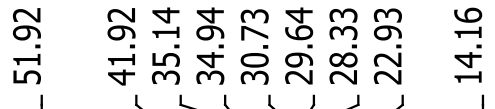

\।14!

${ }^{13} \mathrm{C}-\mathrm{NMR}$ of $( \pm)-7 \mathbf{q}$ in $\mathrm{CDCl}_{3}$
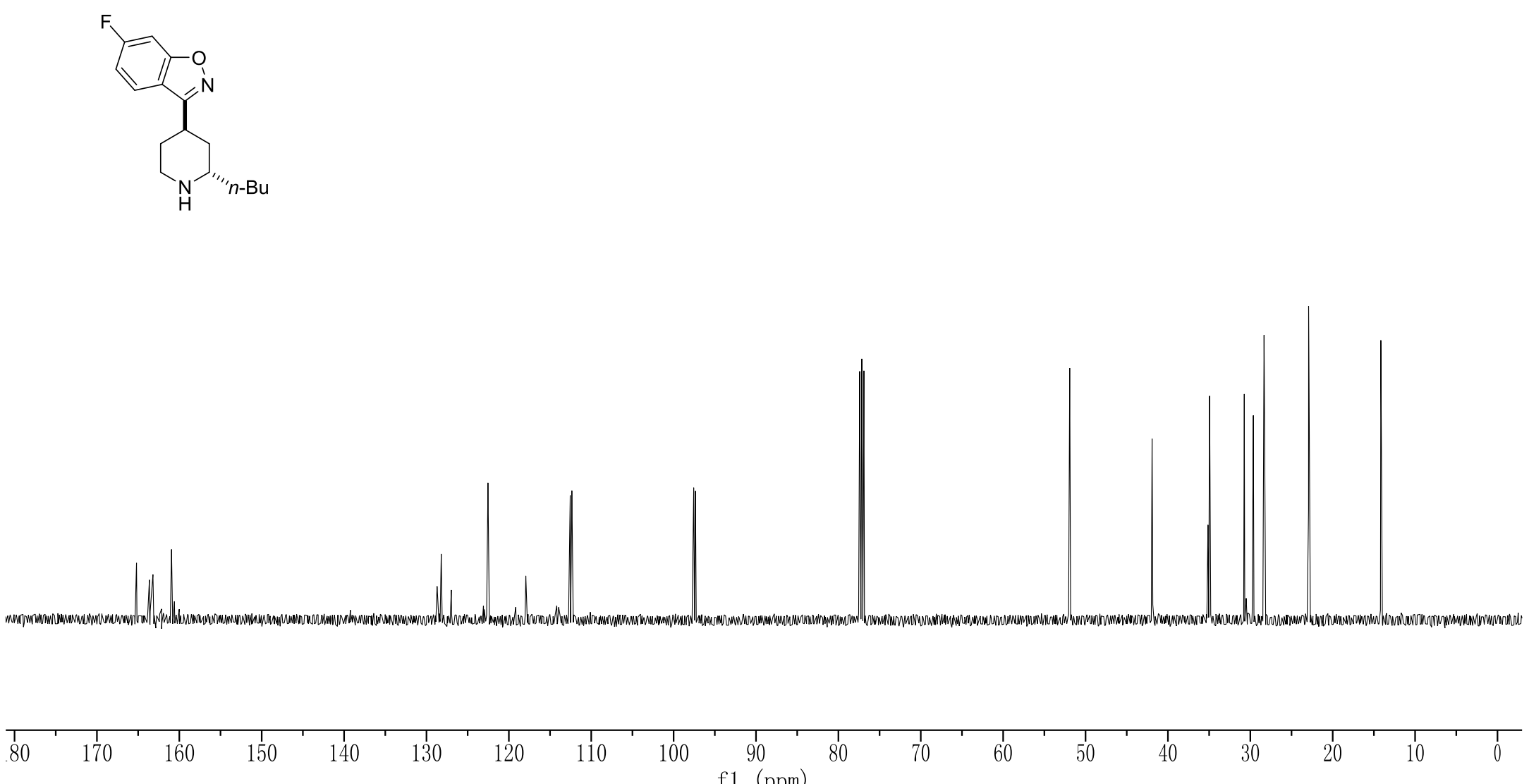


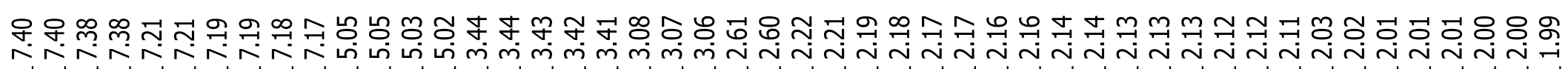

${ }^{1} \mathrm{H}-\mathrm{NMR}$ of $( \pm)-8 \mathbf{a}$ in $\mathrm{CDCl}_{3}$
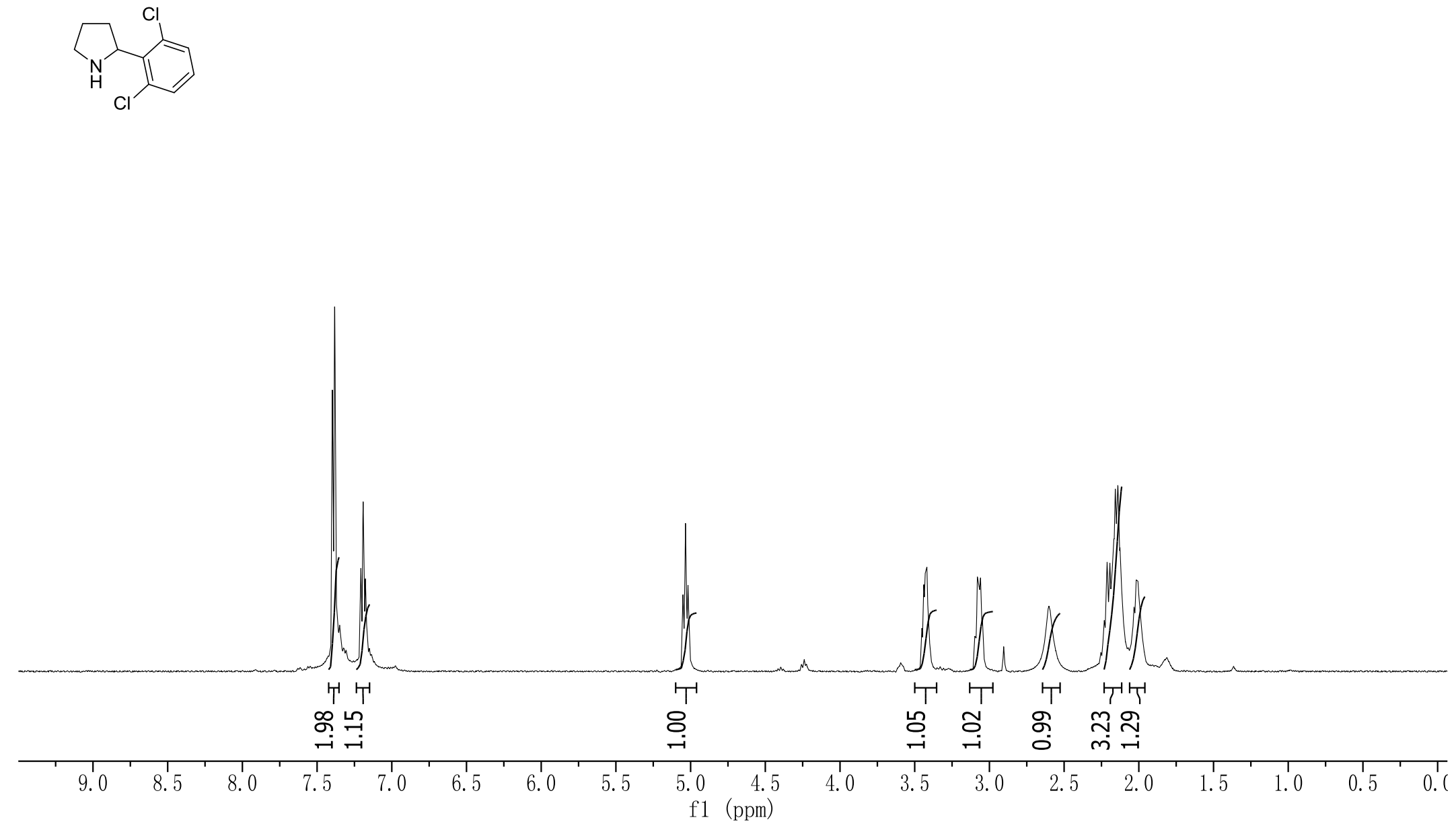


|

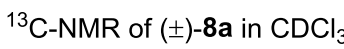

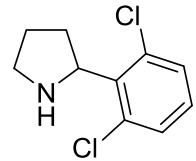

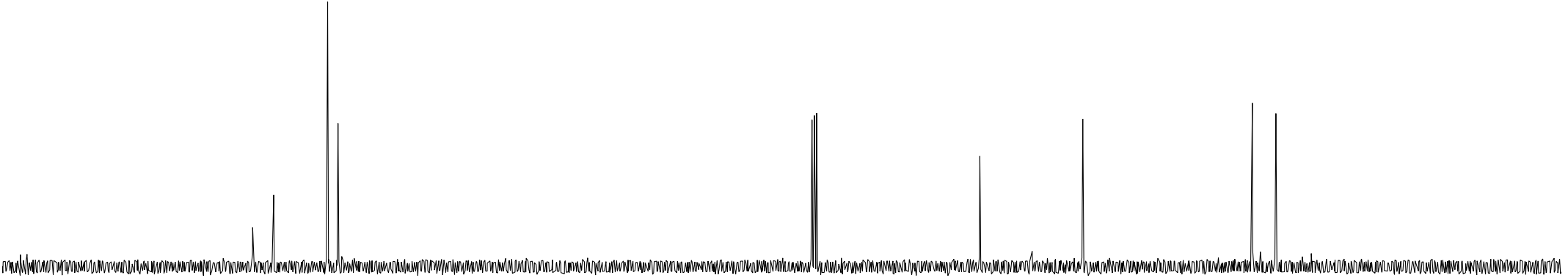

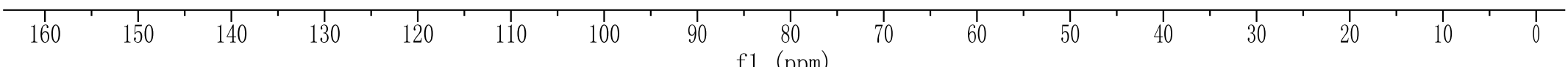




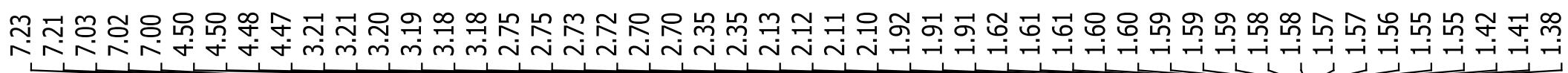

${ }^{1} \mathrm{H}-\mathrm{NMR}$ of $( \pm)-8 \mathbf{b}$ in $\mathrm{CDCl}_{3}$
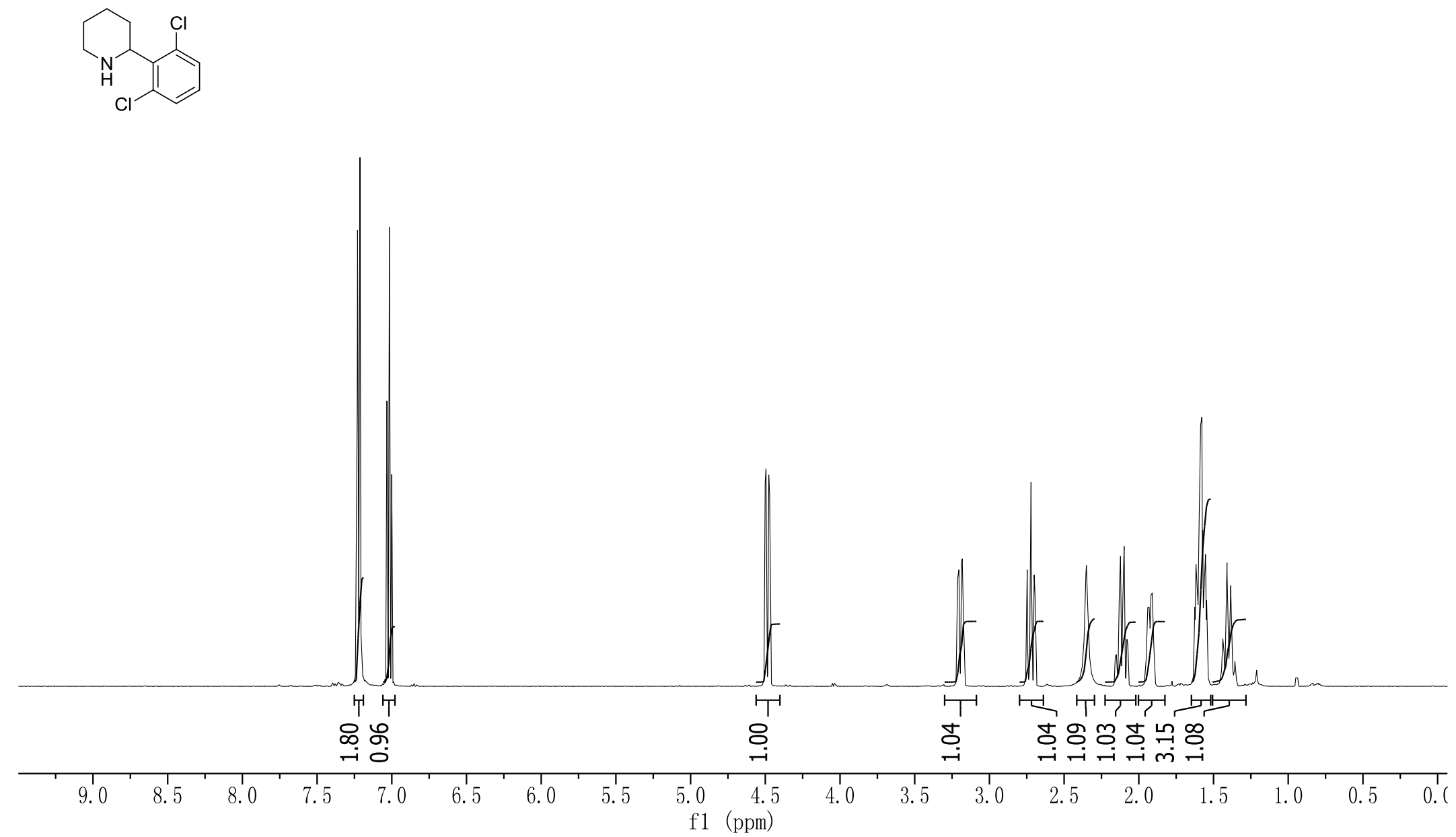


\section{웅요요

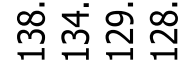 \\ | $|>|$}

${ }^{13} \mathrm{C}-\mathrm{NMR}$ of $( \pm)-8 \mathbf{b}$ in $\mathrm{CDCl}_{3}$

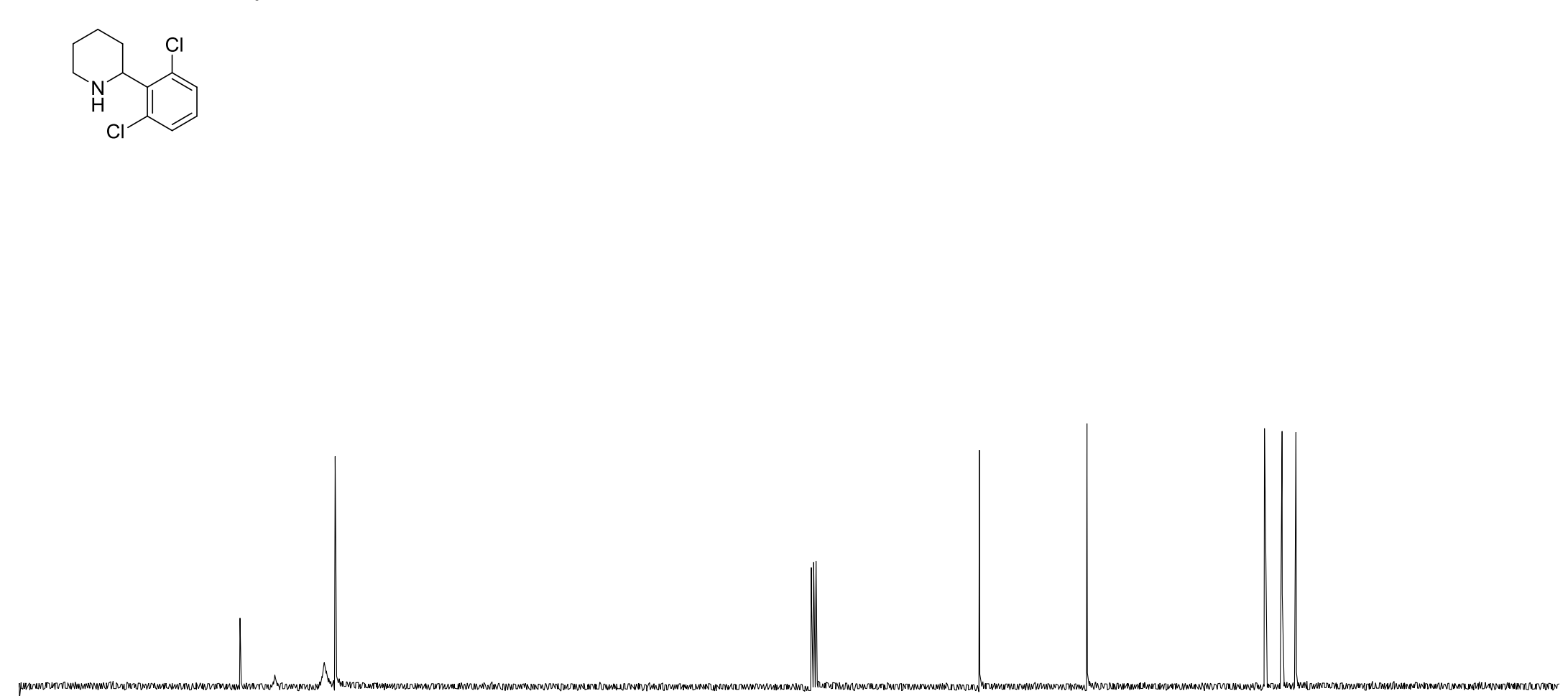

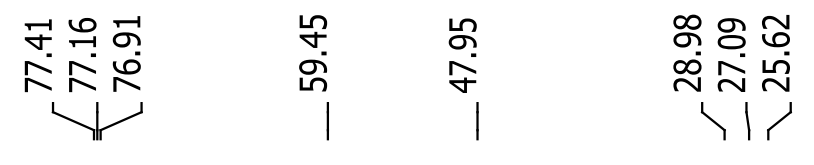

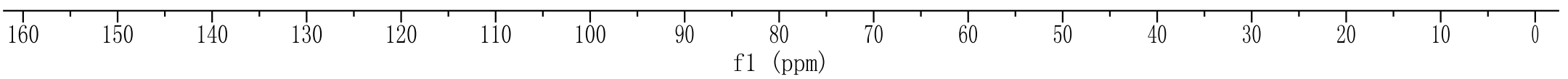




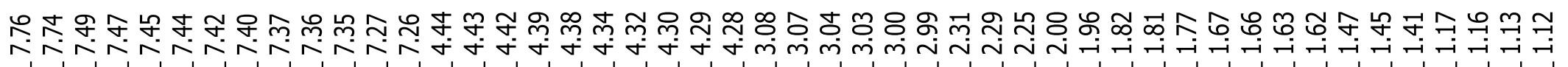

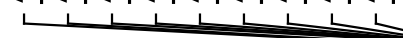

${ }^{1} \mathrm{H}-\mathrm{NMR}$ of $( \pm)-8 \mathbf{c}$ in $\mathrm{CDCl}_{3}$
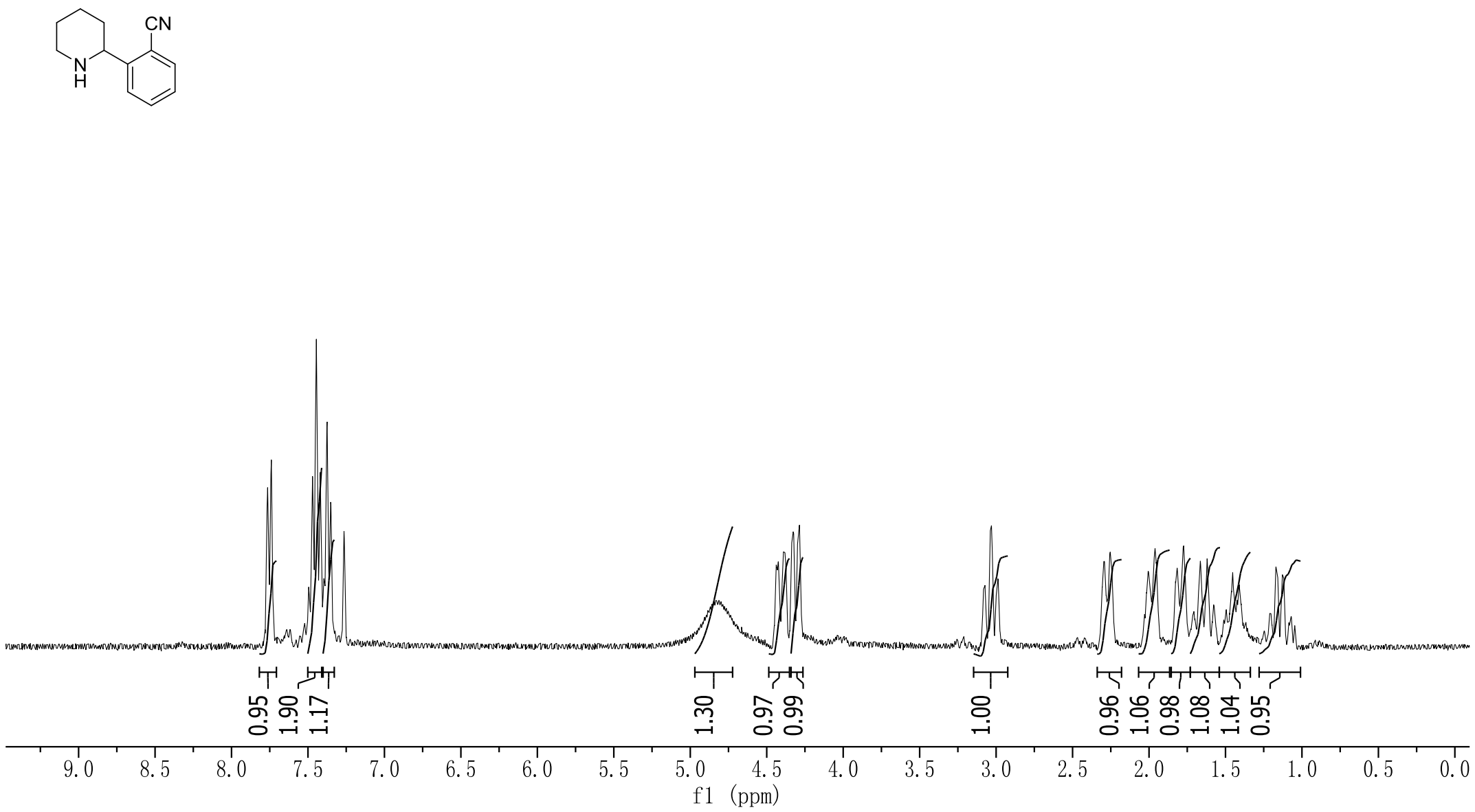


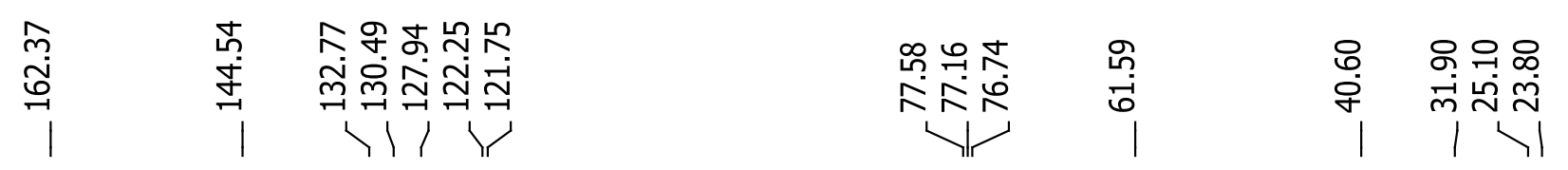

${ }^{13} \mathrm{C}-\mathrm{NMR}$ of $( \pm)-8 \mathrm{c}$ in $\mathrm{CDCl}_{3}$

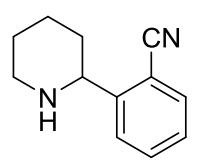

||$_{\text {ind }}$

\begin{tabular}{|c|c|c|c|c|c|c|c|c|c|c|c|c|c|c|c|c|c|}
\hline 18 & 170 & 160 & & & 130 & 120 & 110 & 100 & \multicolumn{2}{|c|}{$\begin{array}{ll}1 & 1 \\
90 & 80\end{array}$} & 70 & 60 & & 40 & 30 & 20 & 10 \\
\hline
\end{tabular}




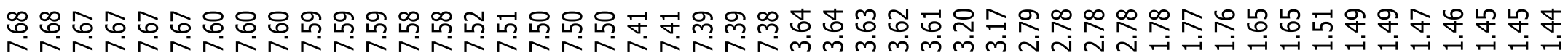

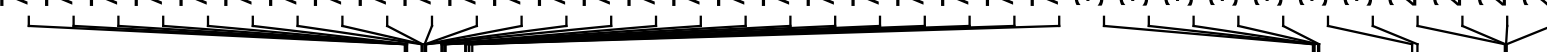

${ }^{1} \mathrm{H}-\mathrm{NMR}$ of $( \pm)-8 \mathbf{d}$ in $\mathrm{CDCl}_{3}$
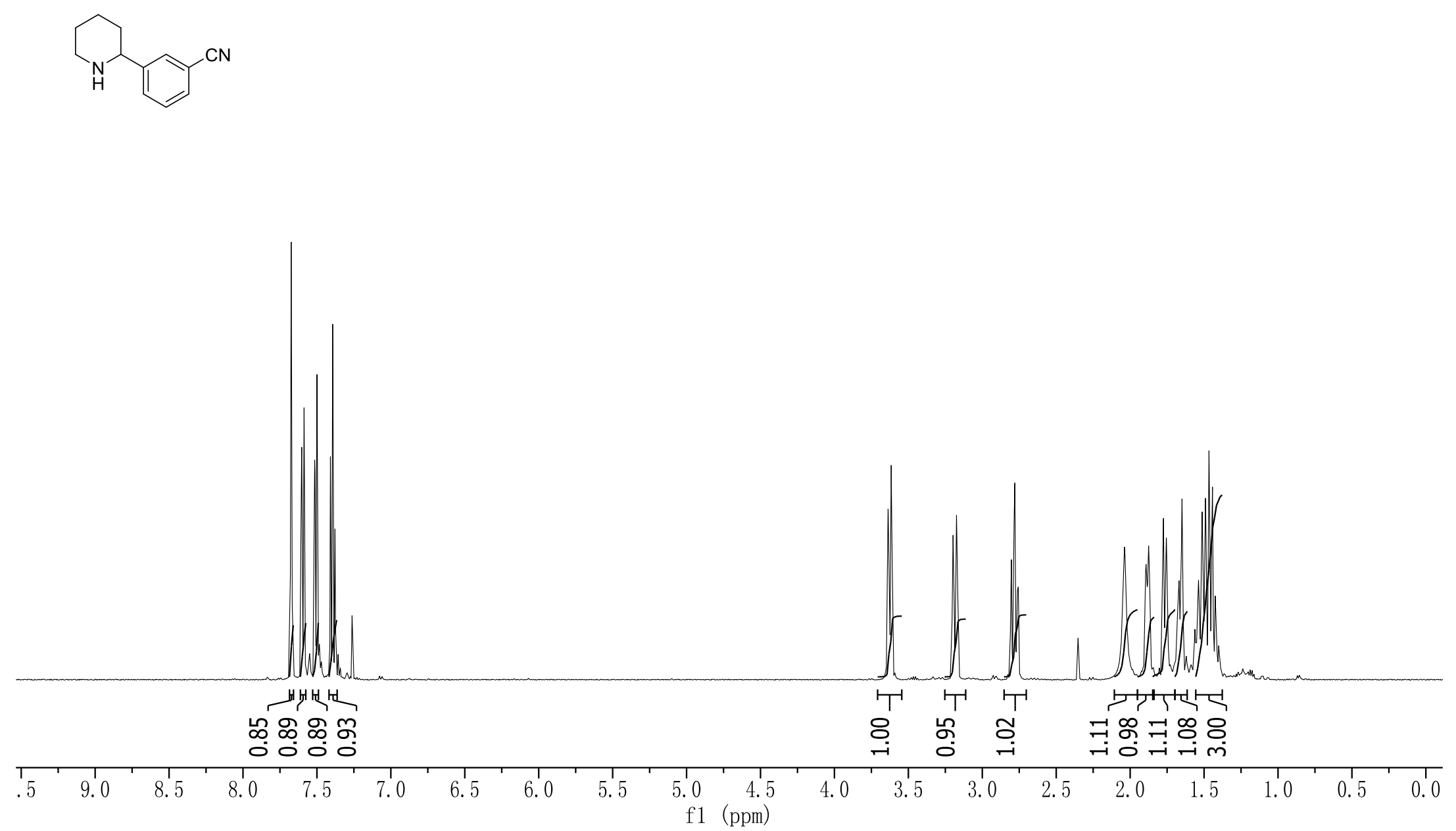


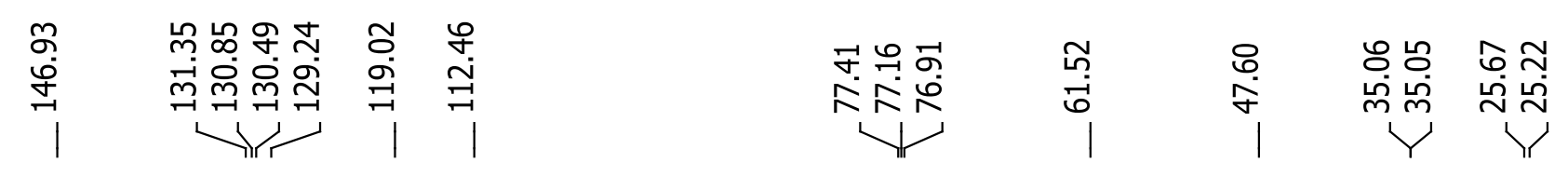

${ }^{13} \mathrm{C}-\mathrm{NMR}$ of $( \pm)-8 \mathrm{~d}$ in $\mathrm{CDCl}_{3}$

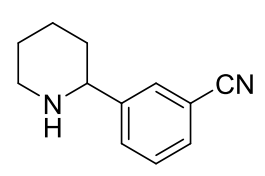

Inmum

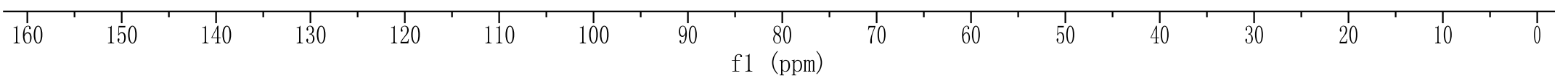




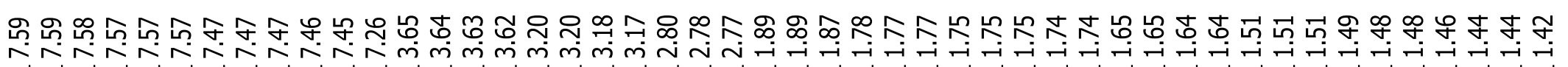
$\longrightarrow$

${ }^{1} \mathrm{H}-\mathrm{NMR}$ of $( \pm)-8 \mathbf{e}$ in $\mathrm{CDCl}_{3}$
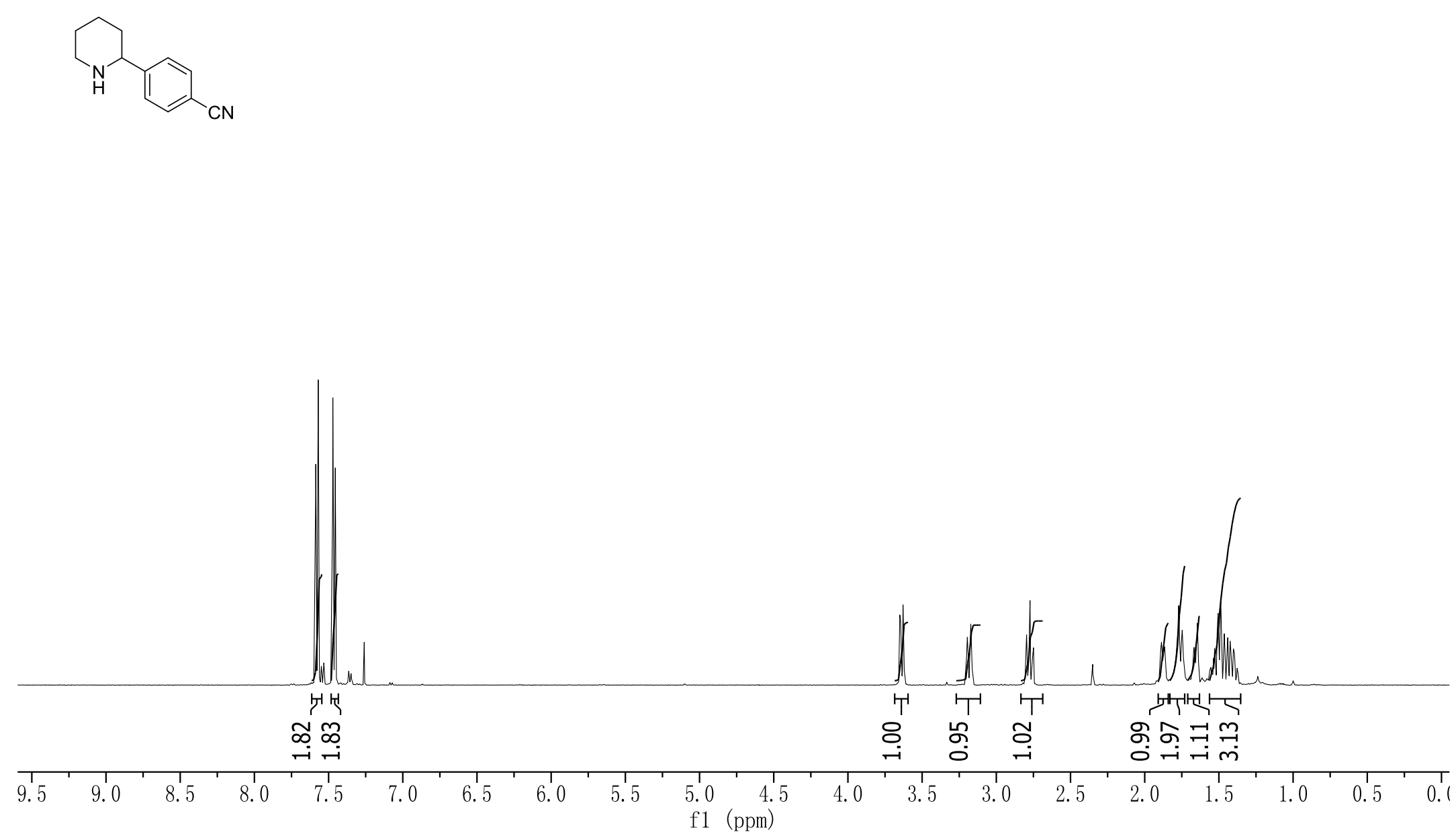


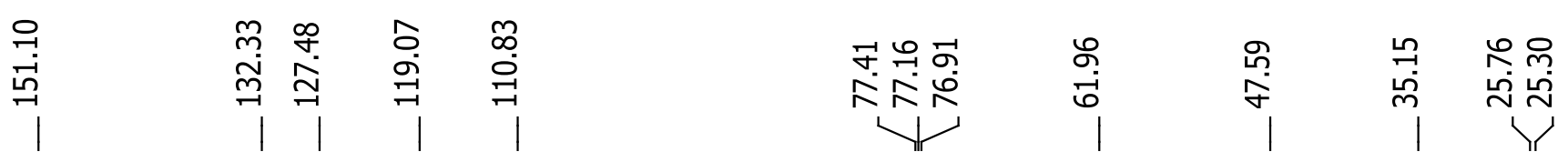

${ }^{13} \mathrm{C}-\mathrm{NMR}$ of $( \pm)-8 \mathrm{e}$ in $\mathrm{CDCl}_{3}$
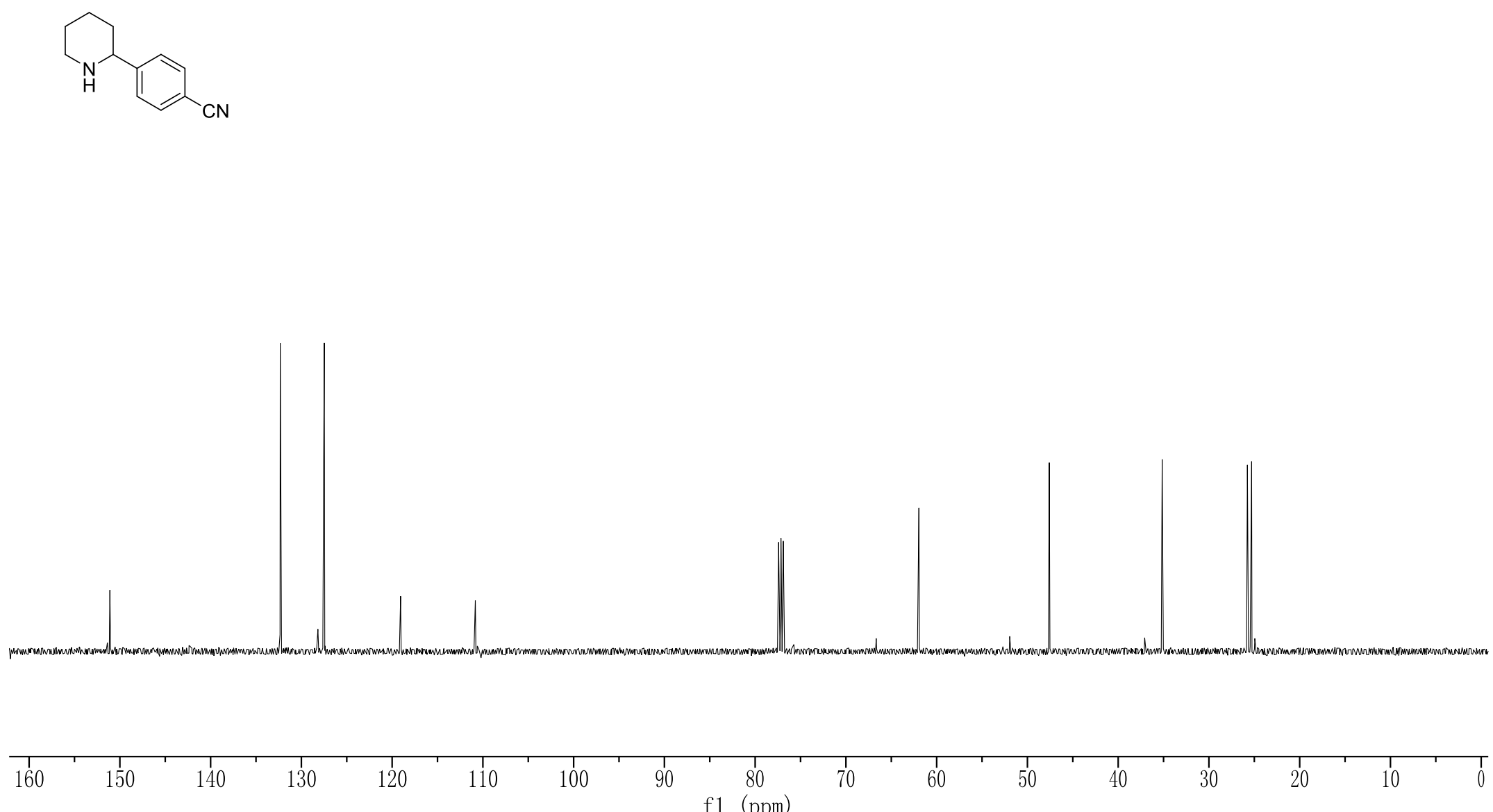
乐

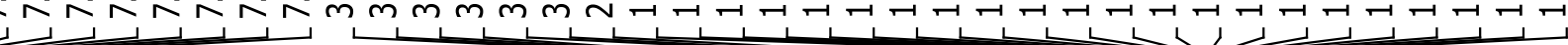

${ }^{1} \mathrm{H}-\mathrm{NMR}$ of $( \pm)-8 \mathrm{f}$ in $\mathrm{CDCl}_{3}$
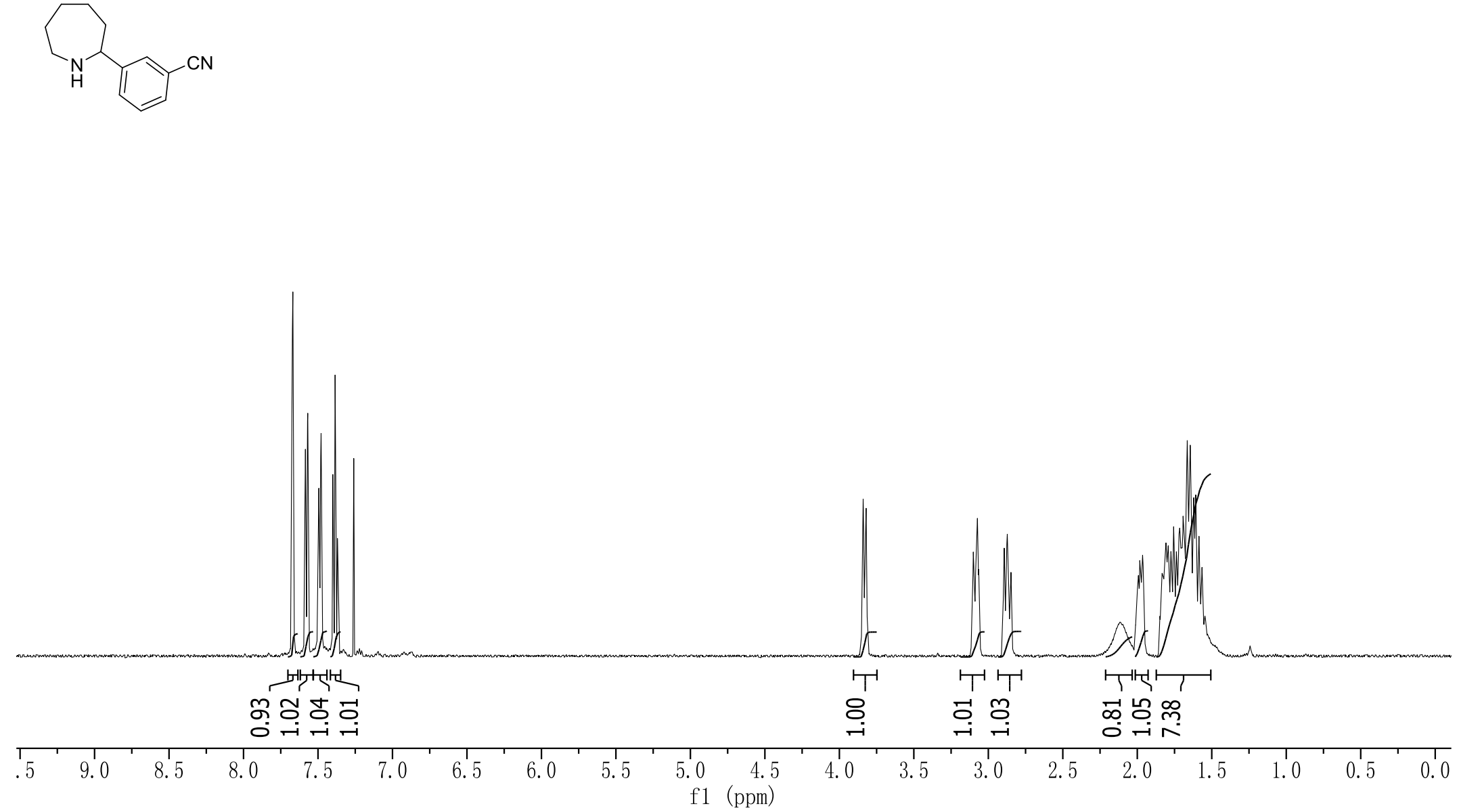


占

${ }^{13} \mathrm{C}-\mathrm{NMR}$ of $( \pm)-8 \mathrm{fin} \mathrm{CDCl}_{3}$<smiles>N#Cc1cccc(C2CCCCCN2)c1</smiles>

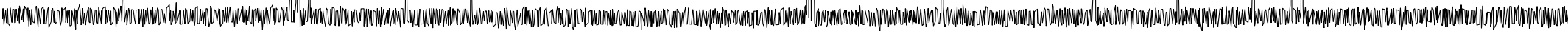

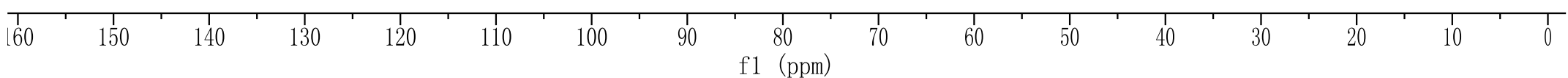




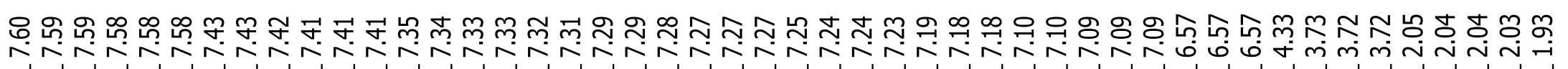

${ }^{1} \mathrm{H}$-NMR of $( \pm)-9$ in $\mathrm{CDCl}_{3}$
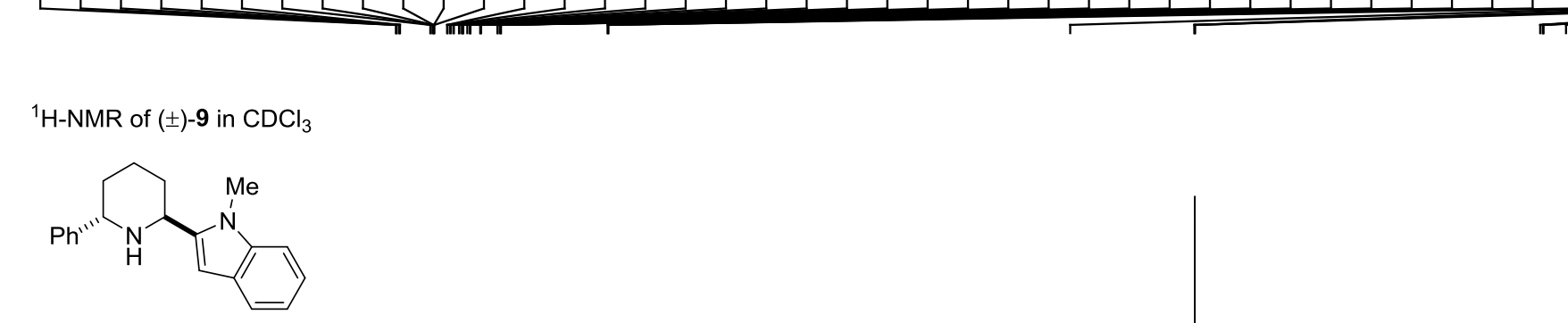


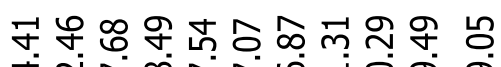

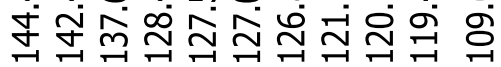

${ }^{3} \mathrm{C}-\mathrm{NMR}$ of $( \pm)-9$ in $\mathrm{CDCl}_{3}$<smiles>Cn1c(C2CCC[C@H](c3ccccc3)N2)cc2ccccc21</smiles>

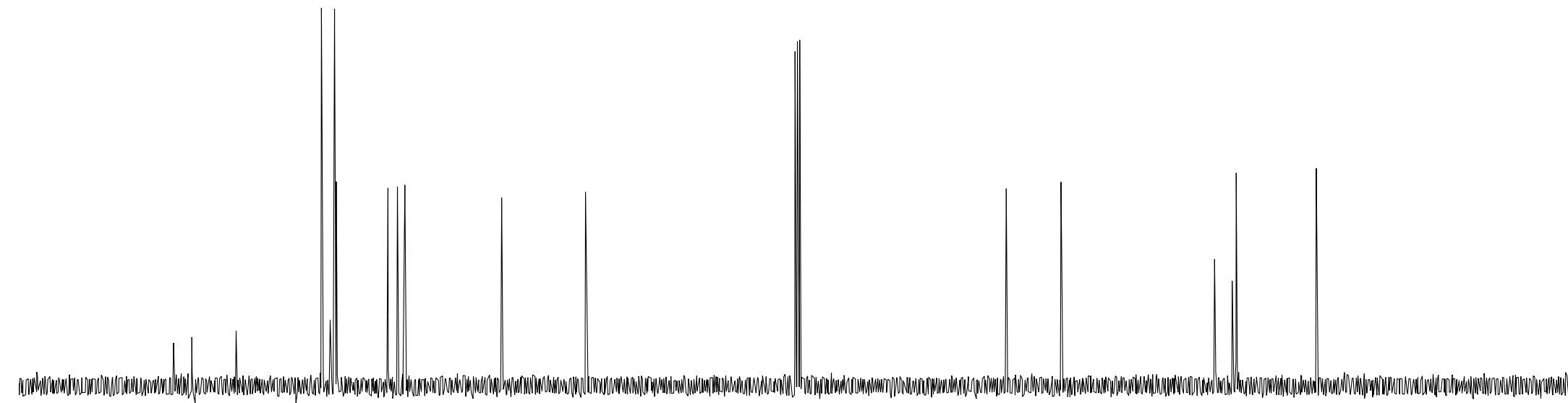

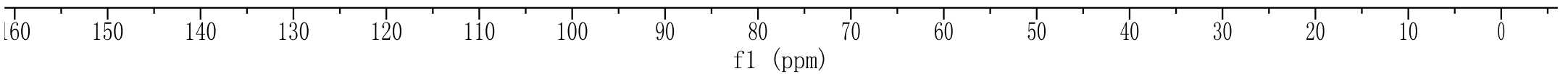




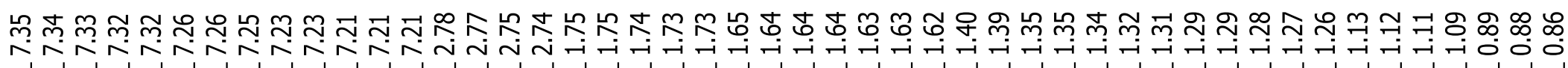

${ }^{1} \mathrm{H}-\mathrm{NMR}$ of $( \pm)-10$ in $\mathrm{CDCl}_{3}$

$\sim_{\mathrm{H}}$

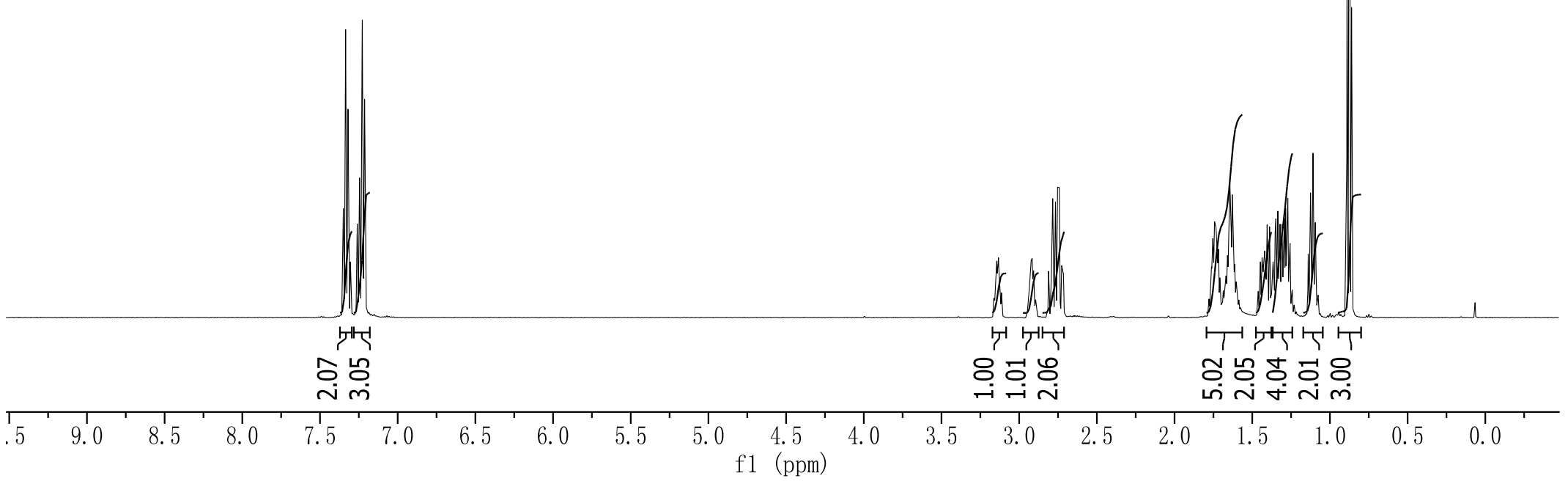




$$
\begin{aligned}
& \text { 용 윤듀 }
\end{aligned}
$$

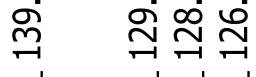

$$
\begin{aligned}
& \text { । रा। }
\end{aligned}
$$

구누요

추

|
菓

กิ่

II

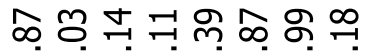

아메

'ए। 1।

${ }^{13} \mathrm{C}-\mathrm{NMR}$ of $( \pm)-10$ in $\mathrm{CDCl}_{3}$
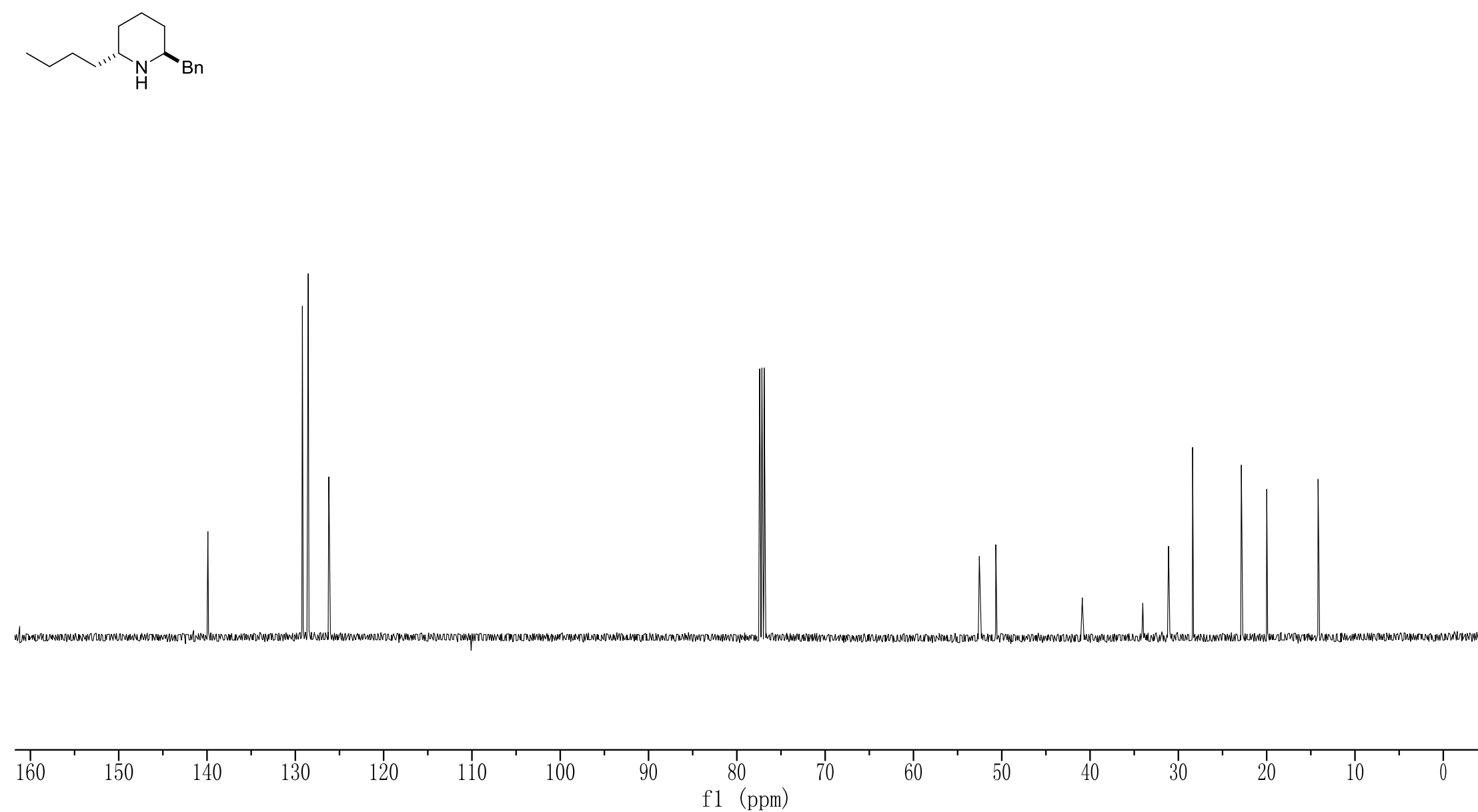


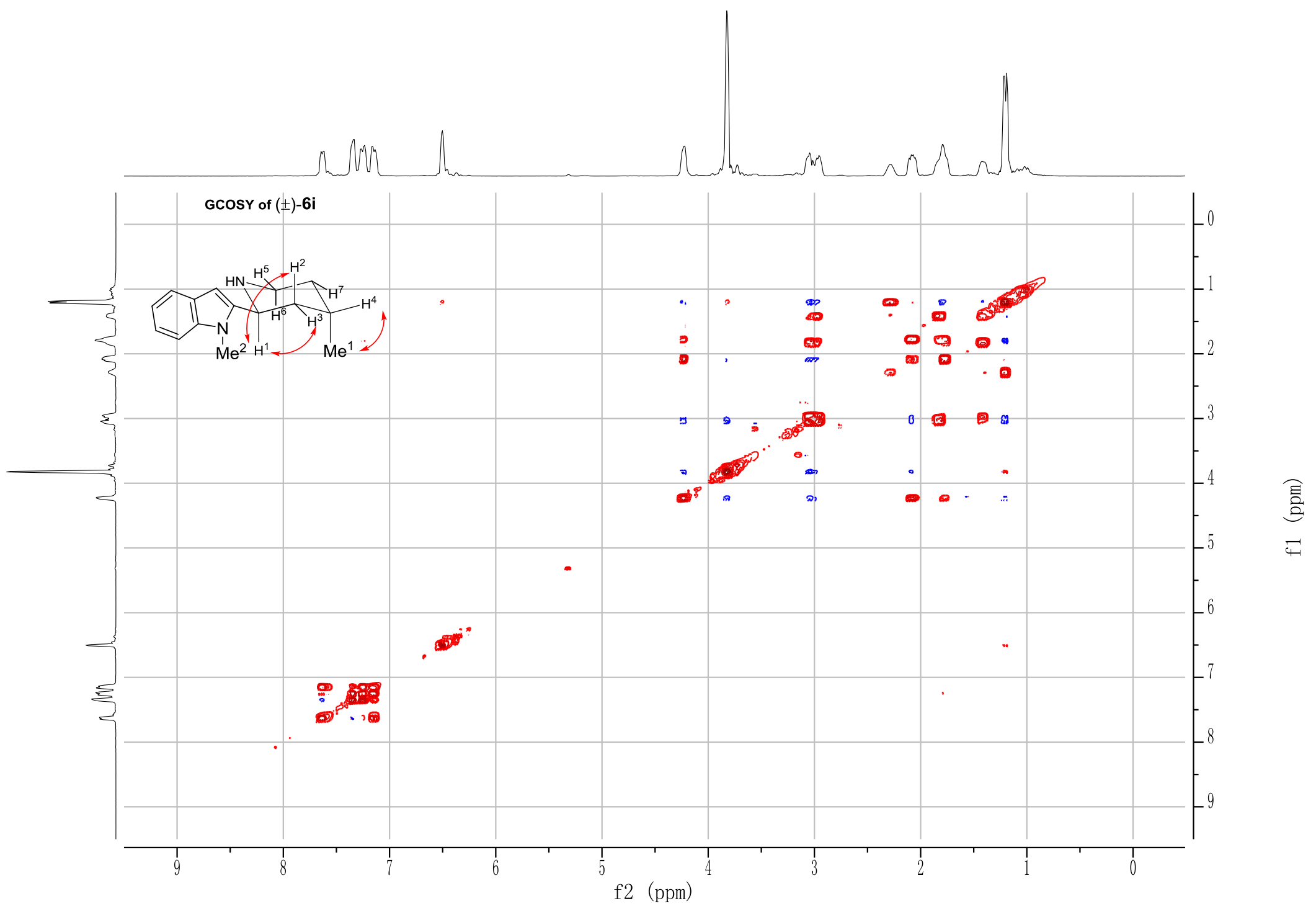




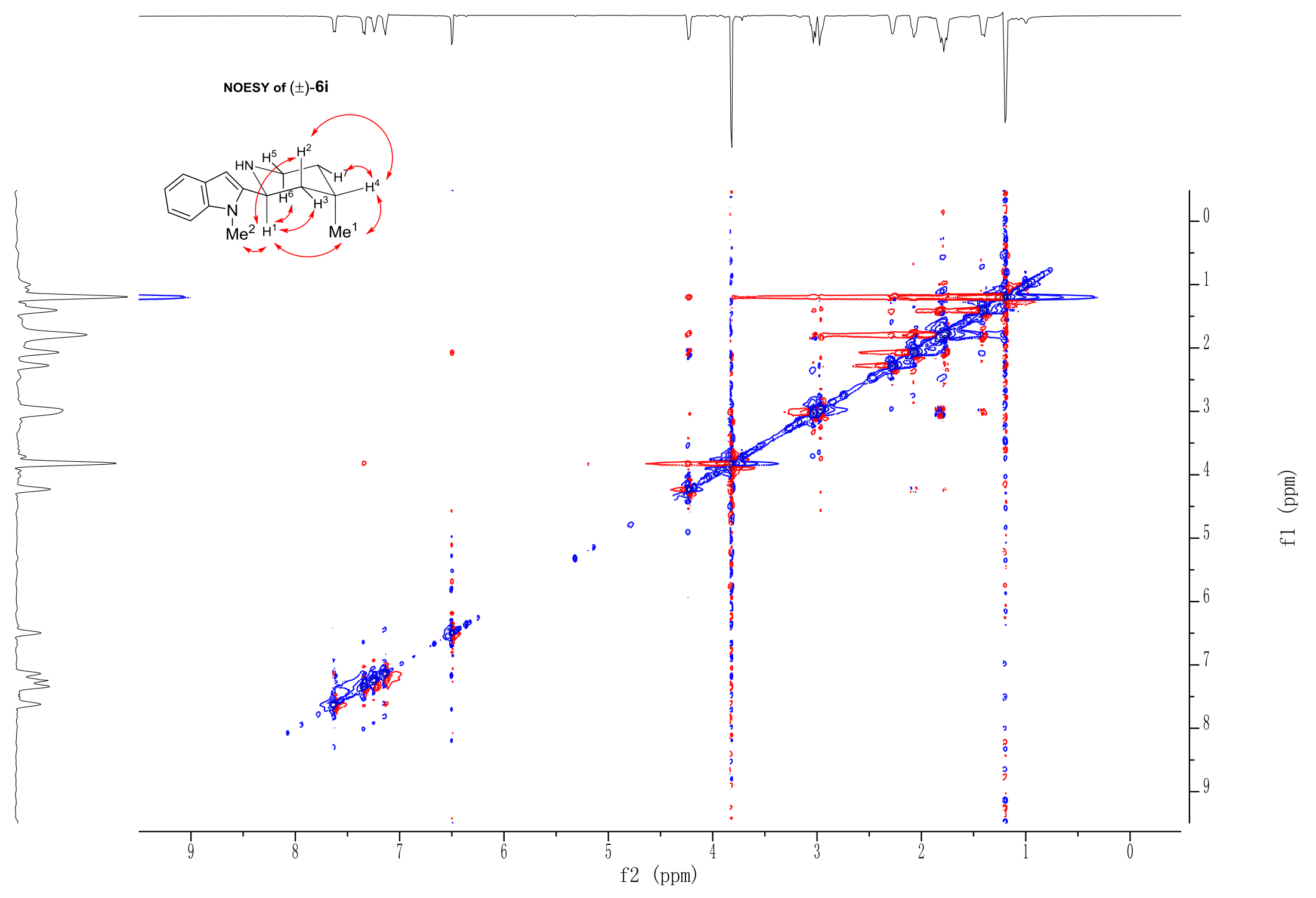

